\title{
Development and implementation of strategies to improve octanoic acid production in Saccharomyces cerevisiae
}

\author{
Dissertation \\ zur Erlangung des Doktorgrades \\ der Naturwissenschaften \\ vorgelegt beim Fachbereich Biowissenschaften \\ der Johann Wolfgang Goethe-Universität \\ in Frankfurt am Main \\ von \\ Leonie Baumann \\ aus München
}

Frankfurt am Main, 2021

(D 30) 

vom Fachbereich Biowissenschaften

der Johann Wolfgang Goethe-Universität

als Dissertation angenommen

Dekan: $\quad$ Prof. Dr. Sven Klimpel

1. Gutachter: Prof. Dr. Eckhard Boles

2. Gutachterin: Prof. Dr. Claudia Büchel

Datum der Disputation: 21. Juni 2021 



\section{This thesis is based on the following publications:}

Leonie Baumann, Arun Rajkumar, John Morrissey, Eckhard Boles \& Mislav Oreb (2018). A yeast-based biosensor for screening of short- and medium-chain fatty acid production. ACS Synthetic Biology 7, 2640-2646. (published)

Leonie Baumann, Florian Wernig, Sandra Born \& Mislav Oreb (2020). Engineering Saccharomyces cerevisiae for production of fatty acids and their derivatives. In: The Mycota Vol. II: Genetics and Biotechnology, 3rd edition, 339368. Springer, Switzerland. (published)

Leonie Baumann, Tyler Doughty, Verena Siewers, Jens Nielsen, Eckhard Boles \& Mislav Oreb (2021). Transcriptomic response of Saccharomyces cerevisiae to octanoic acid production. FEMS Yeast Research. (accepted)

Leonie Baumann, Stefan Bruder, Johannes Kabisch, Eckhard Boles \& Mislav Oreb (2020). High-throughput screening of an octanoic acid producer strain library enables detection of new targets for increasing titers in Saccharomyces cerevisiae. (submitted, in revision) 



\section{Contents}

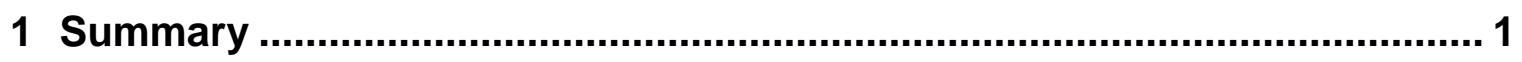

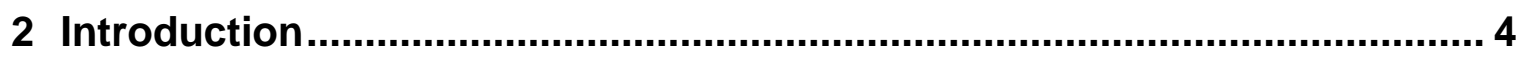

2.1 Fatty acid metabolism in Saccharomyces cerevisiae ........................................ 4

2.1.1 Fatty acid biosynthesis.................................................................. 4

2.1.2 Fatty acid uptake and degradation ................................................. 7

2.2 Metabolic engineering for octanoic acid production in Saccharomyces

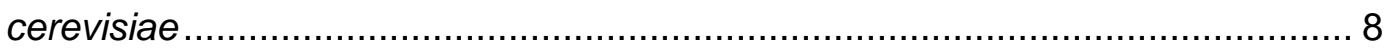

2.2.1 The concept of metabolic engineering ............................................ 8

2.2.2 Yeast as a microbial production host ............................................... 9

2.2.3 Applications of octanoic acid and global market............................... 11

2.2.4 Octanoic acid production in Saccharomyces cerevisiae....................... 13

2.3 Tools for improving octanoic acid production in Saccharomyces cerevisiae ...... 14

2.3.1 Rational engineering ................................................................... 14

2.3.2 Transcriptomics ...................................................................... 16

2.3.3 Adaptive laboratory evolution ................................................. 16

2.3.4 Biosensors and high-throughput screenings ................................. 18

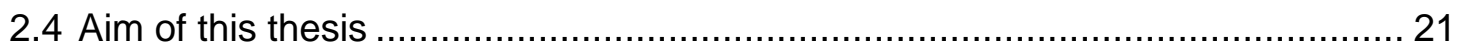

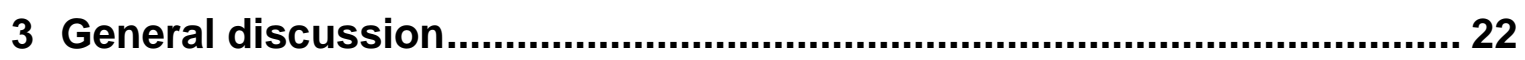

3.1 Octanoic acid biosensor and high-throughput screenings - achievements

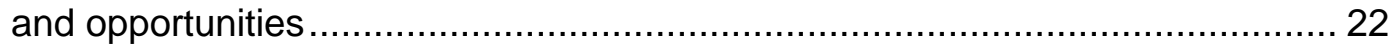

3.2 Saccharomyces cerevisiae physiology during octanoic acid production............. 28

3.3 Strategies to increase strain robustness and reduce octanoic acid toxicity ........ 29

3.4 Future perspectives for octanoic acid production in Saccharomyces cerevisiae 

5.1 Additional results and discussion - Strategies to increase Saccharomyces cerevisiae robustness.

5.1.1 Evaluation of octanoic acid toxicity on Saccharomyces cerevisiae strains

5.1.2 Adaptive laboratory evolution and engineering of identified target genes.

5.1.3 Overexpression of transporters, transcriptional regulators and mutated acetyl-CoA carboxylase

5.1.4 Conclusion. 53

5.2 Material and methods 54

5.2.1 Strains and plasmid construction 54

5.2.2 Cultivation of yeast strains 57

5.2.3 Fatty acid extraction, derivatization and GC-FID analysis 57

5.2.4 Toxicity tests 58

5.2.5 Adaptive laboratory evolution 58

5.2.6 Software 58

5.3 References for the additional results 59

6 Publications 61

6.1 A yeast-based biosensor for screening of short- and medium-chain fatty acid production

6.2 Engineering Saccharomyces cerevisiae for production of fatty acids and their derivatives

6.3 Transcriptomic response of Saccharomyces cerevisiae to octanoic acid production

6.4 High-throughput screening of an octanoic acid producer strain library enables detection of new targets for increasing titers in Saccharomyces cerevisiae..... 136

7 Deutsche Zusammenfassung 



\section{Summary}

Octanoic acid (C8 FA) is a medium-chain fatty acid which, in nature, mainly occurs in palm kernel oil and coconuts. It is used in various products including cleaning agents, cosmetics, pesticides and herbicides as well as in foods for preservation or flavoring. Furthermore, it is investigated for medical treatments, for instance, of high cholesterol levels. The cultivation of palm oil plants has surged in the last years to satisfy an increasing market demand. However, concerns about extensive monocultures, which often come along with deforestation of rainforest, have driven the search for more environmentally friendly production methods. A biotechnological production with microbial organisms presents an attractive, more sustainable alternative.

Traditionally, the yeast Saccharomyces cerevisiae has been utilized by mankind in bread, wine, and beer making. Based on comprehensive knowledge about its metabolism and genetics, it can nowadays be metabolically engineered to produce a plethora of compounds of industrial interest. To produce octanoic acid, the cytosolic fatty acid synthase (FAS) of $S$. cerevisiae was utilized and engineered. Naturally, the yeast produces mostly long-chain fatty acids with chain lengths of C16 and C18, and only trace amounts of medium-chain fatty acids, i.e. C8-C14 fatty acids. To generate an S. cerevisiae strain that produces primarily octanoic acid, a mutated version of the FAS was generated (Gajewski et al., 2017) and the resulting S. cerevisiae FAS ${ }^{\mathrm{R} 1834 \mathrm{~K}}$ strain was utilized in this work as a starting strain.

The goal of this thesis was to develop and implement strategies to improve the production level of this strain. The current mode of quantification of octanoic acid includes labor-intensive, low-throughput sample preparation and measurement a main obstacle in generating and screening for improved strain variants. To this end, a main objective of this thesis was the development of a biosensor. The biosensor was based on the pPDR12 promotor, which is regulated by the transcription factor War1. Coupling $p P D R 12$ to GFP as the reporter gene on a multicopy plasmid allowed in vivo detection via fluorescence intensity. The developed biosensor enabled rapid and facile quantification of the short- and medium-chain fatty acids C6, C7 and C8 fatty acids (Baumann et al., 2018). This is the first biosensor that can quantify externally supplied octanoic acid as well as 
octanoic acid present in the culture supernatant of producer strains with a high linear and dynamic range. Its reliability was validated by correlation of the biosensor signal to the octanoic acid concentrations extracted from culture supernatants as determined by gas chromatography. The biosensor's ability to detect octanoic acid in a linear range of $0.01-0.75 \mathrm{mM}(\approx 1-110 \mathrm{mg} / \mathrm{L})$, which is within the production range of the starting strain, and a response of up to 10-fold increase in fluorescence after activation was demonstrated.

A high-throughput FACS (fluorescence-activated cell sorting) screening of an octanoic acid producer strain library was performed with the biosensor to detect improved strain variants (Baumann et al., 2020a). For this purpose, the biosensor was genomically integrated into an octanoic acid producer strain, resulting in drastically reduced single cell noise. The additional knockout of FAA2 successfully prevented medium-chain fatty acid degradation. A high-throughput screening protocol was designed to include iterative enrichment rounds which decreased false positives. The functionality of the biosensor on single cell level was validated by adding octanoic acid in the range of $0-80 \mathrm{mg} / \mathrm{L}$ and subsequent flow cytometric analysis. The biosensor-assisted FACS screening of a plasmid overexpression library of the yeast genome led to the detection of two genetic targets, $\mathrm{FSH} 2$ and $K C S 1$, that in combined overexpression enhanced octanoic acid titers by $55 \%$ compared to the parental strain. This was the first report of an effect of $F S H 2$ and KCS1 on fatty acid titers. The presented method can also be utilized to screen other genetic libraries and is a means to facilitate future engineering efforts.

In growth tests, the previously reported toxicity of octanoic acid on $S$. cerevisiae was confirmed. Different strategies were harnessed to create more robust strains. An adaptive laboratory evolution (ALE) experiment was conducted and several rational targets including transporter- (PDR12, TPO1) and transcription factorencoding genes (PDR1, PDR3, WAR1) as well as the mutated acetyl-CoA carboxylase encoding gene ACC1S1157A were overexpressed or knocked out in producer or non-producer strains, respectively. Despite contrary previous reports for other strain backgrounds, an enhanced robustness was not observable. Suspecting that the utilized laboratory strains have a natively low tolerance level, four industrial $S$. cerevisiae strains were evaluated in growth assays with octanoic 
acid and inherently more robust strains were detected, which are suitable future production hosts.

The first transcriptomics analysis via RNA-Seq of an $S$. cerevisiae octanoic acid producer strain gave a profound insight into the producer strain's transcriptional response to octanoic acid production in different phases of fermentation (Baumann et al., 2021). The analysis revealed that the transcriptomic response to internally produced octanoic acid is markedly disparate from the responses of wild type strains to externally supplied octanoic acid. In addition, a negative effect of octanoic acid production on strain growth was confirmed. The data suggests that the reduced growth of the octanoic acid producer strain could partly be a result of diminished long-chain fatty acid production. Differential expression analysis of the RNA-Seq data of three sampling times led to the identification of an unreported target, $R P L 40 B$, that when overexpressed increased octanoic acid titers by $40 \%$. This was the first report of Rpl40b to play a role in fatty acid biosynthesis, the exact mechanism of which remains to be investigated.

In summary, in this thesis new strategies were successfully developed and established tools harnessed to improve the yeast $S$. cerevisiae as cell factory for production of octanoic acid. An octanoic acid biosensor was designed, verified, and optimized for implementation in a high-throughput FACS screening. The developed method is highly versatile and can be implemented for various library screenings of short- and medium-chain fatty acid producer strains. A transcriptomics analysis gave a comprehensive picture of strain physiology during octanoic acid production. Overall, three unreported targets were identified and will improve production, ideally in an industrial strain that showed high inherent robustness. 


\section{Introduction}

\subsection{Fatty acid metabolism in Saccharomyces cerevisiae}

Fatty acids play an inevitable role in cells, contributing to many cellular processes such as cell signaling and energy supply. In addition, they serve as building blocks for membrane lipids (phospholipids and sphingolipids) and storage lipids (steryl esters and triacylglycerols). Storage lipids are kept in specialized cellular compartments, so-called lipid droplets, and can be mobilized when necessary as a source of energy for the cells. Cellular fatty acids originate from three sources: uptake from the environment, intracellular lipid turnover and de novo lipid biosynthesis (Tehlivets et al., 2007; Klug \& Daum, 2014).

\subsubsection{Fatty acid biosynthesis}

The de novo lipid biosynthesis pathway is a well-conserved mechanism among eukaryotes and has been studied extensively for several decades. In yeasts such as Saccharomyces cerevisiae, fatty acid biosynthesis takes place in mitochondria and the cytosol. Subsequent modification steps are carried out in the endoplasmic reticulum. Here, we will focus on the cytosolic biosynthesis pathway, which is the main source of cellular fatty acids in the absence of external supply (Tehlivets et al., 2007; Klug \& Daum, 2014). Cytosolic fatty acids are synthesized from the precursor molecules acetyl-coenzyme A (acetyl-CoA) and its derivative malonylCoA by the fatty acid synthase (FAS) as illustrated in Figure 1. Cytosolic acetyl$\mathrm{CoA}$ is generated in the pyruvate dehydrogenase (PDH) bypass pathway from pyruvate produced from glucose (Pronk et al., 1996). The second precursor, malonyl-CoA, is generated via carboxylation of acetyl-CoA, a reaction that is catalyzed by the acetyl-CoA carboxylase Acc1 (Roggenkamp et al., 1980). 


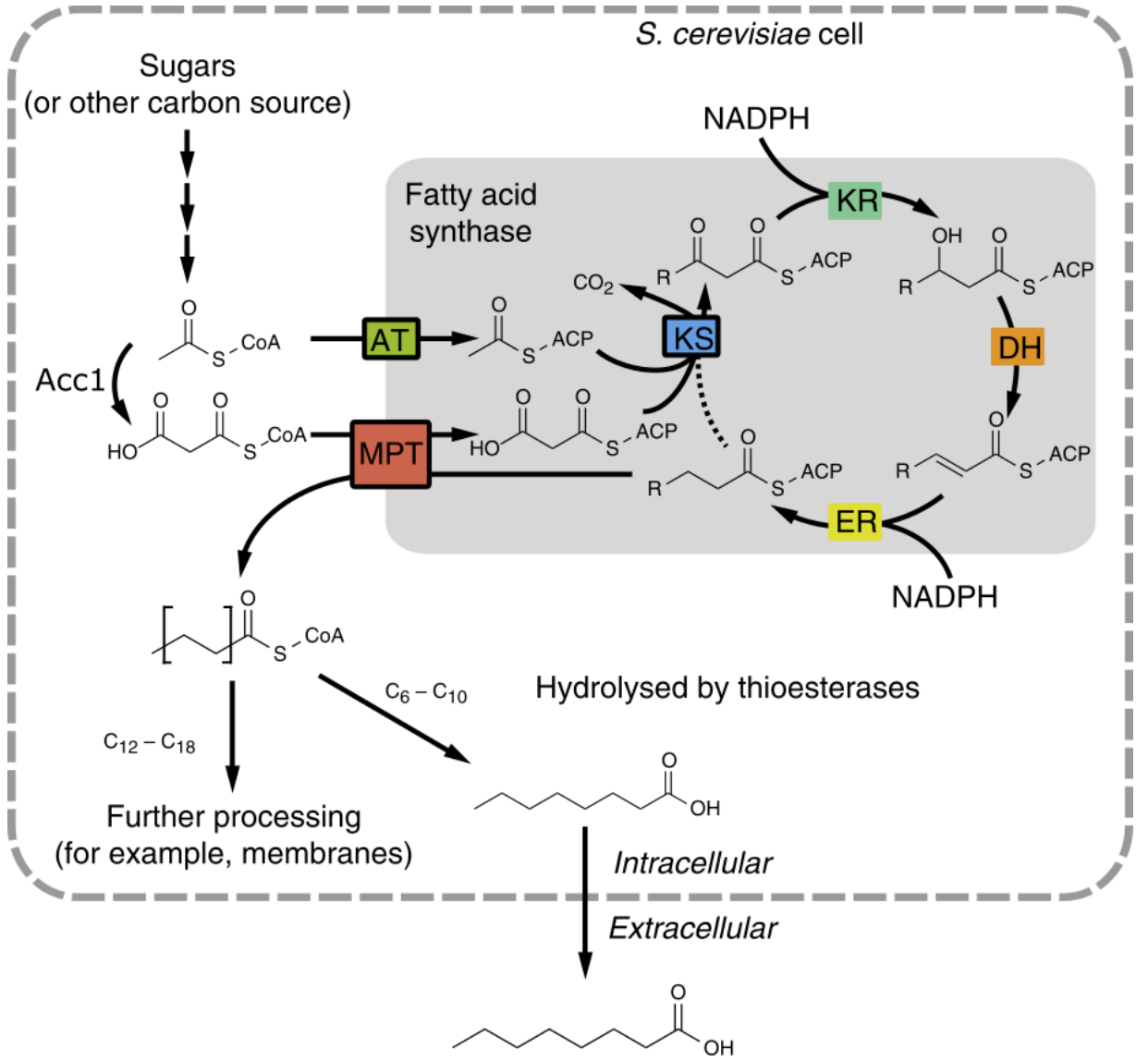

Figure 1: Fatty acid biosynthesis in Saccharomyces cerevisiae. The precursor acetyl-CoA is derived from sugars like glucose and converted to malonyl-CoA by an Acc1-catalyzed carboxylation. The acetyl transferase (AT) domain loads an acyl substrate (starter molecule acetyl or ACP-bound, elongated acyl-chain). A malonyl/palmitoyl transferase (MPT) loads a malonyl molecule which is subsequently bound to the acyl carrier protein (ACP). The two substrates are condensed by the ketoacyl synthase (KS). The resulting $\beta$-ketoacyl intermediate is modified in a series of reaction steps performed by the ketoacyl reductase (KR), dehydratase (DH) and enoyl reductase (ER). The resulting acyl-chain serves as a starter for the next cycle and the process is repeated until the final product is released as an acyl-CoA ester. Acyl-CoAs can be cleaved by thioesterases, releasing free fatty acids. The fatty acid synthesis enzyme can be engineered to produce short- and medium-chain fatty acids (here: C6-C10). Figure modified from Gajewski et al. (2017).

Fatty acids are synthesized by iterative reactions in the so-called "FAS cycle" starting from an acetyl moiety which is successively elongated by the addition of malonyl-CoA molecules. This process is catalyzed by the FAS enzyme, a large complex that consists of six Fas1 and six Fas2 subunits encoded in S. cerevisiae by the genes FAS1 and FAS2, respectively. The FAS complex exhibits different catalytic activities which are embedded in its various domains. Briefly, the single steps of the FAS cycle occur as following: An acetyl transferase (AT) loads an acyl substrate which can be the priming molecule acetyl-CoA or an ACP (acyl carrier protein)-bound, elongated acyl-chain. A malonyl/palmitoyl transferase (MPT) loads 
a malonyl molecule which is subsequently bound to the ACP. These two substrates are condensed by the ketoacyl synthase (KS). The resulting molecule, a $\beta$-ketoacyl intermediate bound to the $\mathrm{ACP}$, is further processed in a series of reaction steps performed by the domains ketoacyl reductase $(\mathrm{KR})$, dehydratase $(\mathrm{DH})$ and enoyl reductase $(E R)$ to a fully reduced acyl-chain. The two reduction steps catalyzed by $\mathrm{KR}$ and ER require the cofactor NADPH. The reduced acyl-chain either serves as a starter for the next cycle or is released as the final product. The last step, the unloading of the final product, is performed by the MPT (Gajewski et al., 2017; Heil et al., 2019).

The main products of the yeast cytosolic FAS cycle are saturated C16 and C18 fatty acids with a minor fraction constituted of saturated C14 fatty acids. A part of the synthesized fatty acids are modified by desaturation, hydroxylation and elongation in other cellular organelles (Klug \& Daum, 2014). The majority of saturated $\mathrm{C} 16$ and $\mathrm{C} 18$ fatty acids undergo desaturation in the endoplasmic reticulum catalyzed by Ole1, resulting in monounsaturated C16:1 (palmitoleic acid) and C18:1 (oleic acid) fatty acids (Viljoen et al., 1986; Stukey et al., 1989, 1990; Tuller et al., 1999). Furthermore, a fraction of the synthesized fatty acids is elongated to very long-chain fatty acids up to C26 by elongases in the endoplasmic reticulum (Toke \& Martin, 1996; Oh et al., 1997).

Fatty acid metabolism is regulated on several levels. The amount of FAS enzyme in the cell is regulated, for instance, on both the gene expression and protein level. Even though Fas1 and Fas2 are present in an equimolar ratio in the FAS enzymatic complex, the overall amount of FAS enzyme is solely determined by the level of Fas1 (Tehlivets et al., 2007). Enhanced Fas1 levels lead to an increase in FAS2 expression, thereby ensuring equimolar amounts of the two parts constituting the complex (Wenz et al., 2001). Apart from the FAS complex, regulation of Acc1 is also critical because malonyl-CoA formation is a rate-limiting step in fatty acid biosynthesis. To adjust cellular malonyl-CoA abundance, regulation on both the transcriptional and protein level is essential (Tehlivets et al., 2007). The expression of $A C C 1$ is controlled by several transcription factors (TFs), e.g. Ino2/Ino4 and Opi1 (Hasslacher et al., 1993; Chirala et al., 1994). On the protein level, Acc1 is inhibited by phosphorylation mediated by the protein kinase Snf1 (Woods et al. 1994). Snf1- 
mediated inhibition was shown to be abolished through mutation of two serine residues of Acc1 (S659A, S1157A), leading to enhanced activity of Acc1 and increased fatty acid production (Choi \& Da Silva, 2014; Shi et al., 2014).

\subsubsection{Fatty acid uptake and degradation}

Besides ne novo biosynthesis, $S$. cerevisiae can also take up externally supplied fatty acids (Klug \& Daum, 2014). The biochemical mechanisms behind fatty acid uptake are poorly understood. There are two main theories about how fatty acids cross biological membranes. The first model states that long-chain fatty acids primarily cross membranes by diffusion which is driven by concentration gradients. According to this theory, fatty acids bind to the membrane and flip through the lipid bilayer without any transporters involved. Many research efforts, however, have also brought evidence for the second scenario (Claus et al., 2019). According to this model, fatty acids mainly cross the plasma membrane by protein-facilitated transport (Higgins, 1994; Zou et al., 2002; Tarling et al., 2013). This mechanism allows for a selective exchange of compounds with the external environment. Furthermore, selective transport can be tightly controlled, thereby avoiding toxic effects, e.g. caused by the accumulation of toxic molecules. Possibly, the mechanism of uptake is dual, depending on the cell's requirements and fatty acid availability (Claus et al., 2019).

Internalized or synthesized free fatty acids are readily activated to their acyl-CoA form, which is mediated in S. cerevisiae by the fatty acyl-CoA synthetases Faa1, Faa2, Faa3, Faa4 or Fat1. Activated fatty acids are either used for the synthesis of triacylglycerols and steryl esters for storage in lipid droplets or degraded by $\beta$ oxidation in the peroxisomes. Long-chain fatty acids are activated primarily by Faa1 and Faa4 prior to transport into the peroxisomes by the transporter Pxa1Pxa2 (Hettema et al., 1996; Scharnewski et al., 2008). In contrast, medium-chain fatty acids enter peroxisomes presumably by passive diffusion or spontaneous flipping with the aid of the auxiliary proteins Ant1 and Pex11 (van Roermund et al., 2000, 2001) and are activated thereafter by peroxisomal Faa2 (Knoll et al., 1994; Hettema et al., 1996). The peroxisomal fatty acid degradation process in $S$. cerevisiae is a cyclic mechanism catalyzed by three enzymes, Pox1, Fox2 and Pot1. In each degradation cycle one acetyl-CoA is released and this process is 
repeated until complete degradation of the fatty acid is achieved (Hiltunen et al., 2003).

\subsection{Metabolic engineering for octanoic acid production in Saccharomyces cerevisiae}

The FAS biosynthesis in $S$. cerevisiae can be modified via metabolic engineering to shift the product spectrum from primarily long-chain fatty acids, i.e. $>\mathrm{C} 16$, to short- and medium chain fatty acids, i.e. C6-C14. The medium-chain fatty acid octanoic acid (C8 fatty acid) is a compound of high industrial interest.

\subsubsection{The concept of metabolic engineering}

The aim of metabolic engineering is to modify the metabolic network of a microorganism to use it for the biotechnological production of pharmaceuticals, chemicals, foods, or biofuels (Woolston et al., 2013). To generate such a microbial cell factory, endogenous metabolic pathways can be improved and rewired, or entirely new pathways are established. Both approaches require a modulation of e.g. gene expression. Considering that the entire cellular network of a microorganism plays a role in the formation of products from substrates, this task can be rather challenging. Targeted genes not only include metabolic genes from the desired pathway but also genes encoding enzymes involved in interconnected reactions, pathway regulation or product degradation. Once the desired pathway is established in laboratory-scale in the host organism, the product titer, yield and productivity need to be maximized which is achieved through iterative rounds of the so-called "design-build-test" cycle. Eventually, the goal of every metabolic engineering effort is a cost-effective production of the target compound in industrial scale (Woolston et al., 2013; Nielsen \& Keasling, 2016).

The biotechnological production of chemicals and biofuels can be a sustainable alternative to current production methods, i.e. petrochemistry or extraction from plants (Saling, 2005). One of the great advantages of a biotechnological production is that renewable resources, for instance sugars like glucose, xylose or arabinose, can be used as starting material. Nowadays, most commercial biotechnological processes utilize sugars derived from sugar cane or starch. However, to prevent 
competition with food production, the use of non-edible waste materials like lignocellulose, which accumulates in other industrial production processes as side product, is increasingly investigated (Gustavsson \& Lee, 2016).

The metabolic engineering field has developed at an incredible speed in the last three decades. This is the result of a plethora of new emerging technologies which at the same time have become more easily available, faster and cheaper. The most important developments include chemical DNA synthesis, which allows for the expression of heterologous genes in any host organism, the advances in whole genome sequencing, and the rapid increase of omics-based tools as well as highthroughput screening methods (Woolston et al., 2013). In addition to that, genetic engineering tools like the CRISPR/Cas system (Jinek et al., 2012) have simplified and accelerated genome editing of microorganism. Furthermore, through synthetic biology it was demonstrated that it is possible to create entire synthetic chromosomes (Gibson et al., 2010; Postma et al., 2021). Intertwining metabolic engineering with synthetic biology and a systems biology approach, i.e. considering the cell in its entirety, will likely allow for production of a bigger variety of products and the use of a wider spectrum of microbial hosts in the near future (Nielsen \& Keasling, 2016).

To date, a number of microbial production processes are already commercialized. Some prominent examples are the production of the feed supplement lysine by Corynebacterium glutamicum (Brautaset \& Ellingsen, 2011), the chemical building block 1,4-butanediol via Escherichia coli (Burgard et al., 2016), production of antibiotics with the filamentous fungus Penicillium chrysogenum (van den Berg, 2010) and the production of bioethanol with S. cerevisiae (Mohd Azhar et al., 2017).

\subsubsection{Yeast as a microbial production host}

S. cerevisiae - often simply referred to by "budding yeast" or "baker's yeast" - is a unicellular fungus of the phylum Ascomycota. S. cerevisiae has been employed by mankind for thousands of years for bread baking and production of alcoholic beverages like beer, wine and sake. The molecular basis of these processes is well understood by now and $S$. cerevisiae is the predominant yeast species used in these and other biotechnological processes (Schmelzer et al., 2020). One of the 
most important products in industrial biotechnology is bioethanol, the production of which takes advantage of the baker's yeast intrinsic ability to produce high amounts of ethanol (Mohd Azhar et al., 2017).

However, S. cerevisiae cannot only be used to produce primary metabolites like ethanol, but also for non-inherent products like secondary metabolites and recombinant enzymes by applying metabolic engineering. Roughly $13 \%$ of biopharmaceuticals approved by the European Medicines Agency (EMA) and the US Food and Drug Administration (FDA) were produced in $S$. cerevisiae in the years 2003-2014. In 1982, insulin was the first recombinant protein (produced in $E$. coli) that was licensed as biopharmaceutical for use in humans (Baeshen et al., 2014). Only four years later, in 1986, insulin was successfully produced in $S$. cerevisiae (Markussen et al., 1986; Thim et al., 1986) and nowadays, the producer Novo Nordisk has a worldwide market share of about $50 \%$ with its yeast-produced insulin products (Schmelzer et al., 2020). With globally rising incidence of diabetes, the annual sales of insulin are rapidly increasing (Kaplan et al., 2016; Saeedi et al., 2019) - demonstrating the importance of biotechnological processes for today's society.

With the desire for a bigger variety of biotechnological products, the need for host organisms with very specific characteristics is growing. Therefore, the interest in other, less characterized yeast species has increased in the past years. Some of these so-called non-conventional yeasts have inherent properties that are desirable in industrial production processes (Steensels et al., 2014; Thorwall et al., 2020). The yeast Yarrowia lipolytica, for example, can accumulate more than $50 \%$ of its dry cell weight in the form of lipids, making it a suitable host for bio-oil production (Beopoulos et al., 2009). Kluyveromyces marxianus is a fast-growing, highly thermotolerant yeast, able to use a broad range of carbon substrates - traits that are desirable for industrial fermentations (Cernak et al., 2018). Another example is Kluyveromyces lactis, which produces the enzyme lactase enabling it to degrade the "milk sugar" lactose. K. lactis has been widely used in dairy industry since the 1950s to generate lactose-free products but has also been used as a host for the expression of over 40 heterologous proteins to date (van Ooyen et al., 2006). 
Despite advances in the use of a variety of yeast species for biotechnological purposes, $S$. cerevisiae is still the host-of-choice for most industrial and lab-scale applications (Hong \& Nielsen, 2012). S. cerevisiae was the first eukaryote with sequenced genome in the year 1996, which strongly facilitated metabolic engineering efforts (Goffeau et al., 1996). Today, it is one of the best studied microorganisms with well-understood physiology, genetics and metabolism. $S$. cerevisiae has an inherent ability for homologous recombination which can be combined with an array of molecular and genetic engineering tools that are available today, such as adapted CRISPR/Cas systems (Da Silva \& Srikrishnan, 2012; Generoso et al., 2016). Another advantage is its ease in handling, as well as its FDA-approved GRAS (generally regarded as safe) status (Da Silva \& Srikrishnan, 2012).

\subsubsection{Applications of octanoic acid and global market}

Octanoic acid, also known as caprylic acid, is a saturated medium-chain fatty acid consisting of an eight-carbon backbone and a terminal carboxyl group. It is a colorless oily liquid with a mild, slightly unpleasant odor (PubChem, 2020). Octanoic acid naturally occurs in coconut and palm kernel oil and in some dairy products (Rupilius \& Ahmad, 2007; Deen et al., 2020). The industrial range of applications of octanoic acid is expansive: It is used as antimicrobial in food preservation as well as in household cleaning products, as flavoring agent in baked goods to confer a cheesy and buttery taste, as ingredient in infant formulas and as emulsifier in cosmetics. It is utilized in the synthesis of dyes and drugs and as component of lubricants, pesticides, herbicides and insecticides (PubChem, 2020). Furthermore, a variety of studies have demonstrated possible applications of octanoic acid in medicine. For instance, its antimicrobial properties were shown to be beneficial for treatment of candidiasis infections and it was investigated for the treatment of high cholesterol levels and essential voice tremor (Wilson et al., 2006; Xu et al., 2013; Bae \& Rhee, 2019; Lowell et al., 2019).

The global demand for octanoic acid and thus production have steadily increased over the last years (Yan \& Pfleger, 2020). Octanoic acid is mainly sourced from palm kernel oil and coconut oil, in which it is contained in the form of triglycerides (Berger, 2003; Deen et al., 2020; Yan \& Pfleger, 2020). Approximately $4 \%$ of the 
fatty acids contained in palm kernel oil and $7.5 \%$ in coconut oil constitutes octanoic acid. For the extraction of octanoic acid from these oils, the triglycerides are split into the glycerin backbone and the fatty acids, followed by separation of the different fatty acids of the mixture (Rupilius \& Ahmad, 2007; Deen et al., 2020). The global production volume of palm kernel oil is projected to reach 8.9 million tons and of coconut oil 3.6 million tons in the years 2019/20 (IndexMundi, 2020).

The growing demand for these oils, especially for use as biodiesel, has led to an enhanced production of plant oil crops alongside with rising concerns about the environmental impact (Fargione et al., 2008). In countries like Malaysia and Indonesia, large areas of rainforest are cleared to establish new plantations for palm oil plants, thereby contributing to increasing greenhouse gas emissions. Another problem is the ethical dilemma of cultivating edible crops for use as chemical compounds or biofuels instead of human nutrition (Fargione et al., 2008). In addition to these concerns, the extraction and purification process from plants is complex and associated with excessive water consumption (Schmidt, 2015).

A possible solution to these problems is the biotechnological production of fatty acids through microorganisms like $S$. cerevisiae as schematically depicted in Figure 2.

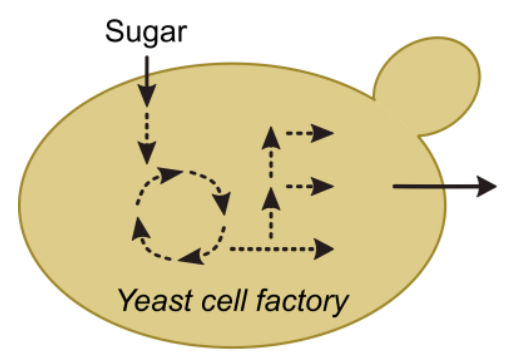

Fatty acids

Fatty alcohols

Fatty aldehydes etc.
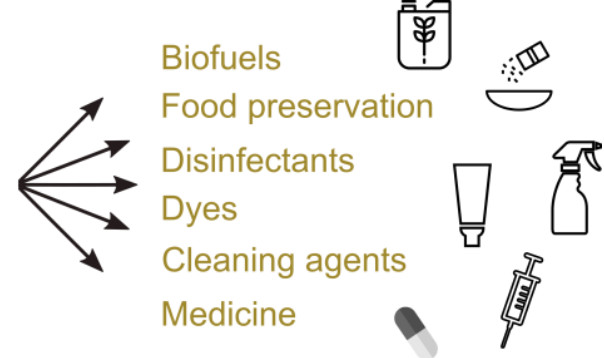

Figure 2: Biotechnological production of fatty acids and derivatives via yeast. Abundant, nonedible materials like lignocellulose can be used as sugar source for a microbial conversion to valuable compounds. Yeast is used as a "cell factory" that produces fatty acids, alcohols, aldehydes and other derivatives which find application in various consumer products.

A biotechnological approach allows for the production of highly specific compounds, thereby avoiding complex purification processes. Furthermore, fatty acids can be microbially converted to derivatives like fatty alcohols (biofuels) or other chemical compounds widely used in industry, e.g. fatty acid esters, aldehydes and alkanes/alkenes (Pfleger et al., 2015; Baumann et al., 2020b). Most 
importantly, the microbial production approach can reduce the motivation for converting tropical rainforest into farmland, as non-edible waste materials can be used as starting material to feed the microbe (D'Espaux et al., 2017).

\subsubsection{Octanoic acid production in Saccharomyces cerevisiae}

Naturally, S. cerevisiae mainly produces $\mathrm{C} 16$ and $\mathrm{C} 18$ fatty acids with minor amounts of $\mathrm{C} 14$ and $\mathrm{C} 26$; but production of medium-chain fatty acids like octanoic acid is rare and therefore requires metabolic engineering. There are different strategies to establish short- and medium-chain fatty acid production in $S$. cerevisiae. Several studies have shown that expression of bacterial or human FAS systems combined with short- and medium-chain specific heterologous thioesterases, which cleave ACP-bound fatty acyl chains, enables increased release of short- and medium-chain fatty acids (Leber \& Da Silva, 2014; Fernandez-Moya et al., 2015; Zhu et al., 2017). Another strategy is to manipulate chain-length control of the native yeast FAS, and relies on site-directed mutagenesis of the FAS-encoding genes. Gajewski et al. (2017) applied this approach to reduce the malonyl affinity of the MPT domain of Fas1. Reduced malonyl uptake supposedly favors the release of incompletely elongated acyl chains. This rational was successfully demonstrated by the exchange of an arginine by a lysine residue (R1834K) in the MPT domain, leading to production of short- and medium-chain fatty acids, in particular octanoic acid (Gajewski et al., 2017). Such a manipulated FAS enzyme is a good basis for an S. cerevisiae chassis strain for octanoic acid production.

To advance octanoic acid titers, several bottlenecks need to be addressed. These include the supply of adequate amounts of precursors, i.e. acetyl-CoA and malonylCoA, co-factors (NADPH), and energy (ATP), minimizing degradation of the product, and maximizing excretion of the product. Another issue that needs to be considered is the toxicity of octanoic acid for S. cerevisiae. 


\subsection{Tools for improving octanoic acid production in Saccharomyces cerevisiae}

Key requirements for increasing octanoic acid production in $S$. cerevisiae is a detailed knowledge about the cell's metabolism as well as good tools for manipulation. Even though some knowledge has been gathered, there are still many unknows concerning the cell's metabolism during fatty acid production. Rational engineering to establish an octanoic acid production pathway in $S$. cerevisiae was already successful and some improvements in the biosynthesis could be achieved; however, classical tools have reached their limits and need to be complemented with new strategies to reach industry-scale titers and yields (Nielsen \& Keasling, 2016; Baumann et al., 2020b).

\subsubsection{Rational engineering}

Rewiring of the cellular metabolism is accomplished traditionally through rational engineering, thereby shifting the flux towards a desired product, in this case medium-chain fatty acids. Rational engineering relies on a broad knowledge of the cellular network, enzyme kinetics, pathway regulation as well as possible bottlenecks. Commonly applied methods include knockout, overexpression and targeted mutation of metabolic genes (Woolston et al., 2013).

Significant efforts have been devoted to improving the precursor supply routes to provide carbon, energy and redox power and an extensive outline of strategies can be found in the attached publication in chapter 6.2 (Baumann et al., 2020b). Synthesis of the precursor acetyl-CoA in the yeast cytosol via the PDH bypass route is uneconomical for overproduction of fatty acids (van Rossum et al., 2016). Therefore, research has focused on establishing other, more energy-efficient routes for acetyl-CoA supply, e.g. by introducing heterologous pathways into $S$. cerevisiae - with mixed success rates (Baumann et al., 2020b). Acc1 has been another prominent target for rational engineering since it provides malonyl-CoA (chapter 2.1.1 and below). Apart from precursor supply, the FAS enzyme complex has also been rationally engineered. The beforementioned mutations in relevant domains (e.g. R1834K in the MPT domain) led to a drastically altered fatty acid profile. Furthermore, it was reported that a fused version of the $\alpha$ and $\beta$ subunits of 
FAS, namely fusFAS, showed superior performance (Wernig et al., 2020). Another common method is the overexpression of rate-limiting enzymes. For instance, the exchange of the native FAS promoters by the strong constitutive promoters pTDH3, pTEF1 and truncated $p H X T 7$ increased final octanoic acid titers (Wernig et al., 2020).

To prevent octanoic acid degradation, i.e. chain length specific $\beta$-oxidation, gene knockouts were used. In particular knockout of FAA2, encoding a short- and medium-chain specific fatty acyl-CoA synthetase, led to strongly increased octanoic acid titers (Leber et al., 2016; Henritzi et al., 2018).

Two rational strategies have been employed to counteract octanoic acid toxicity on yeast. Octanoic acid leads to a disruption of the plasma membrane composition, causing membrane leakage and in certain concentrations cell death (Legras et al., 2010; Liu et al., 2013). These effects were to a certain extent reduced by altering the plasma membrane composition via enhanced oleic acid supply. The oleic acid content in the membrane was increased by external supply or via engineered Acc1 ${ }^{\text {S1157A }}$. The higher tolerance towards octanoic acid was attributed to an increase in the average chain length of membrane fatty acids and higher cismonounsaturated fatty acid levels (Liu et al., 2013; Besada-Lombana et al., 2017).

Taken together, these rational approaches allowed to produce octanoic acid at titers ranging in the order of $\mathrm{mg} / \mathrm{L}$ - amounts that are insufficient for an industrialscale production. The applied methods are centered around an in-depth understanding of the fatty acid biosynthesis pathway as well as its associated fluxes and regulatory system. However, many genes and enzymes that are connected to fatty acid biosynthesis, degradation or robustness in the framework of global regulatory networks are unknown. Oftentimes, multiple enzymes are involved in a poorly understood manner in generating a certain phenotype. This impedes the use and impact of rational approaches (Woolston et al., 2013). To unravel the role of such enzymes and connections, combinatorial approaches and new strategies are necessary. 


\subsubsection{Transcriptomics}

In a transcriptomics analysis the entirety of a cell's transcriptome is identified and quantified. This not only allows for a global analysis of gene expression at a snapshot in time but also of changes in the expression profile under different conditions. The method has become highly popular over the last years as a result of improved technology in combination with decreased costs. The prevalent technique used today is RNA-Seq, i.e. next-generation sequencing of the transcriptome. RNA-Seq analysis can elucidate the producer strain's physiology during production of the compound-of-interest and factors can be detected which were previously unknown to play a role in the biosynthesis (Wang et al., 2009; McGettigan, 2013). For instance, RNA-Seq analysis has been used to study $S$. cerevisiae's response to the production of fatty alcohols (Dahlin et al., 2019) and cyanobacterial response to free fatty acid production (Ruffing, 2013), revealing condition-specific responses of the host organisms.

Two transcriptome-wide studies analyzed a wild type strain's response to externally supplied octanoic acid. The strains were supplemented with $0.05 \mathrm{mM}$ octanoic acid (7 mg/L) for 20 minutes and $0.3 \mathrm{mM}$ octanoic acid (43 mg/L) until mid-log growth, respectively (Legras et al., 2010; Liu et al., 2013). The first study by Legras et al. (2010) revealed that the transporters Pdr12 and Tpo1 are involved in octanoic acid expulsion and that the yeast showed an oxidative stress response. The second study by Liu et al. (2013) suggested that octanoic acid supply leads to membrane leakiness and thereby cell disruption. The group demonstrated that oleic acid supplementation alleviated this toxic effect. Both studies revealed important response mechanisms to supplied octanoic acid, however, they did not analyze the transcriptomics of an S. cerevisiae octanoic acid producer, which might strongly differ.

\subsubsection{Adaptive laboratory evolution}

For microbial adaptive laboratory evolution (ALE), microorganisms are cultivated and growth is monitored in a controlled laboratory setting for long periods of time, usually in the range of several weeks to months. The cells are put under pressure by environmental stress factors like high temperature, low $\mathrm{pH}$, or the presence of 
toxic compounds, which reduce growth rates. Having to cope with this pressure, some cells develop improved phenotypes, which accumulate in the cell population during long term selection. The underlying basis of such phenotypic changes are genetic mutations and selection for improved traits (Dragosits \& Mattanovich, 2013). The steps of an ALE experiment are schematically shown in Figure 3.

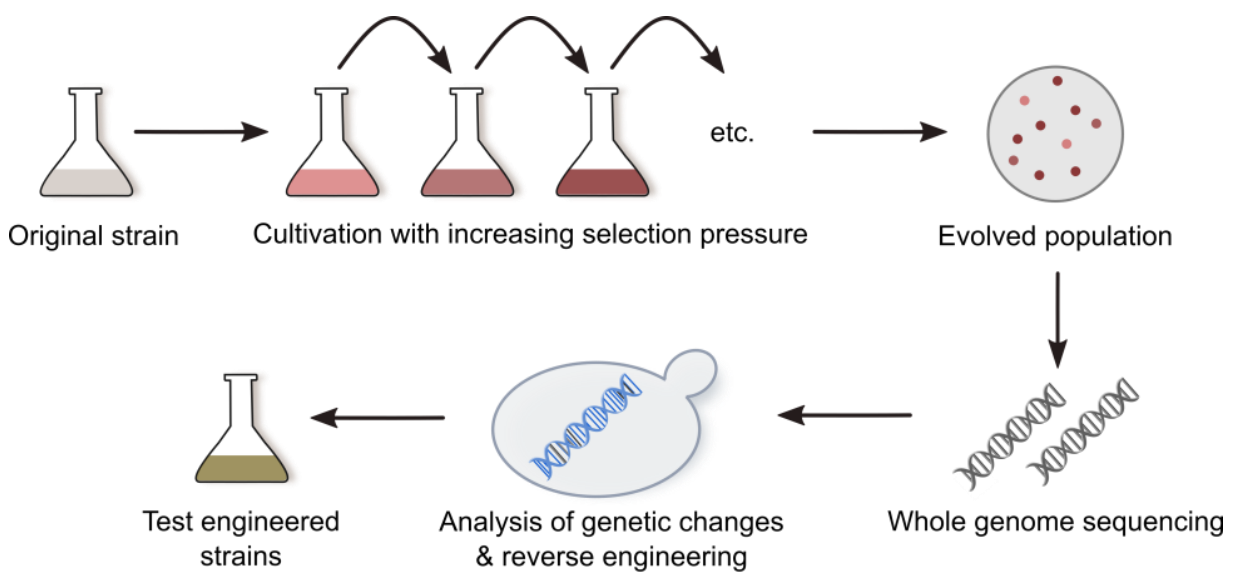

Figure 3: Adaptive laboratory evolution (ALE). A microbial strain is cultivated over several generations in serial shake flask cultures with a selection pressure such as increasing toxic compound concentration or decreasing $\mathrm{pH}$. Evolved populations include individual cells with improved phenotypes. The genome of selected cells is sequenced, genetic changes identified and reversely engineered into the original strain, resulting in improved strain performance.

The common laboratory procedure for ALE is a serial batch cultivation in multiple shake flasks. An aliquot of the cell culture is transferred to a new flask with fresh medium at regular intervals and the serial transfers are repeated until a desired phenotype - usually improved growth - is observable. Eventually, single cells are extracted from the culture and genetic changes can be analyzed by whole genome re-sequencing. This allows for easy and rapid phenotype-genotype correlations. Common changes that can be observed after an ALE experiment are singlenucleotide polymorphisms (SNPs), small-scale insertions and deletions (indels) as well as deletions and duplications of larger genomic regions up to entire chromosomes. By reversely engineering these changes into the starting strain through classical genetic methods, identified targets can be validated, and a superior, more robust production strain can be generated (Dragosits \& Mattanovich, 2013; Mans et al., 2018). 
ALE has been used to select cells with an enhanced tolerance to an array of industrially relevant compounds. For instance, ALE of $E$. coli with high concentrations of octanoic acid resulted in evolved strains with changes in membrane composition and fluidity. These changes conferred enhanced tolerance to octanoic acid as well as increased production titers (Royce et al., 2015). ALE has also been performed with $S$. cerevisiae, for example for increased resistance to acetic acid (González-Ramos et al., 2016) and medium-chain alcohols (González-Ramos et al., 2013; Davis López et al., 2018). The artificial evolution of microorganisms in combination with trait analysis and subsequent reverse engineering of mutations into production hosts, is a vital tool for future cell factory development. It can enable yeast to catabolize substrates which are found in renewable resources like lignocellulose or to tackle toxicity issues which are encountered in the production of fatty acids and biofuels (Abatemarco et al., 2013; Mans et al., 2018). An inherent limit of ALE is that it relies on easily detectable phenotypes like increased growth. Although some phenotypes, like stress tolerance, are tied to growth, it is difficult to couple the production, and hence titers of molecules like fatty acids, to growth. Therefore, it is also necessary to develop additional screening and selection strategies to further improve fatty acid production (Nielsen \& Keasling, 2016).

\subsubsection{Biosensors and high-throughput screenings}

To quantify fatty acids produced by a microbial strain, they must be extracted, undergo methylation to fatty acid methyl esters (FAMEs) and subsequently be analyzed by chromatography. Sample preparation for FAME quantification from each individual culture is a laborious, time-consuming procedure and analysis relies on low-throughput chromatography-based methods (Michener et al., 2012; Schallmey et al., 2014; Nielsen \& Keasling, 2016).

An elegant solution to circumvent this issue is a biosensor. Biosensors can detect small molecules in the cell and respond by regulating host processes. Hence, biosensors can be utilized as devices for sensitive and specific small-molecule detection, thereby substantially speeding up detection and quantification. Genetic elements that are commonly used as biosensors are transcription factors (TFs). TFs respond to one or more inducing molecules by targeting promoters with 
specific recognition sites, thereby dynamically regulating gene expression. When coupling TF-responsive promoters to a gene encoding for a fluorescent protein, the presence of a molecule is transformed into an easily detectable, quantifiable output. Such TF-based biosensors can be applied in metabolic engineering to livemonitor titers or even dynamically regulate production pathways (Schallmey et al., 2014; D'Ambrosio \& Jensen, 2017). Concerning fatty acid production in $S$. cerevisiae, TF-based biosensors have been applied to monitor and regulate supply of the precursor malonyl-CoA. For this purpose, several research groups have adapted the bacterial FapR-fapO system to yeast, which enabled sensing of intracellular malonyl-CoA levels ( $\mathrm{Li}$ et al., 2015; David et al., 2016). Another approach is the use of the bacterial transcriptional repressor FadR. FadR is a ligand-responsive TF that controls the expression of several genes involved in fatty acid biosynthesis, degradation and transport (Zhang et al., 2012). FadR represses transcription in the absence of long-chain fatty acids/acyl-CoAs. By coupling FadRresponsive operator sites to synthetic yeast promoters and a GFP-encoding gene, an S. cerevisiae biosensor mainly responsive to $\mathrm{C} 14$ fatty acid was generated (Teo et al. 2013).

Biosensors for intracellular molecules are valuable tools, especially to monitor pathway intermediates and to determine rate-limiting enzymes. These biosensors, however, do not serve for determining the overall produced amount of a compound when it is secreted from the cell. This is the case for many small molecules of industrial interest, including octanoic acid, which is readily secreted to the environment. To sense extracellular octanoic and decanoic acid, Mukherjee et al. (2015) expressed heterologous G-protein coupled receptors (GPCRs) in yeast. These receptors were linked to the yeast mating pathway for signaling and GFP expression as an output. Unfortunately, the linear and dynamic ranges of the biosensors were low and they were not proven to be functional in culture broth (Mukherjee et al., 2015), which would be indispensable for a useful biosensor.

Functional biosensors facilitate rapid and multiplexed phenotypic evaluation of cells. Therefore, biosensors can be used in high-throughput screenings of genetically diverse populations consisting of up to $10^{9}$ clone variants to detect bestperforming strains (Schallmey et al., 2014). The common experimental setup is 
schematically shown in Figure 4. Strain libraries can be generated by a plethora of ways, for instance, by random mutagenesis with UV-radiation, error prone PCR, promoter shuffling of pathway genes, or plasmid cloning of genome-wide libraries (D’Ambrosio \& Jensen, 2017).

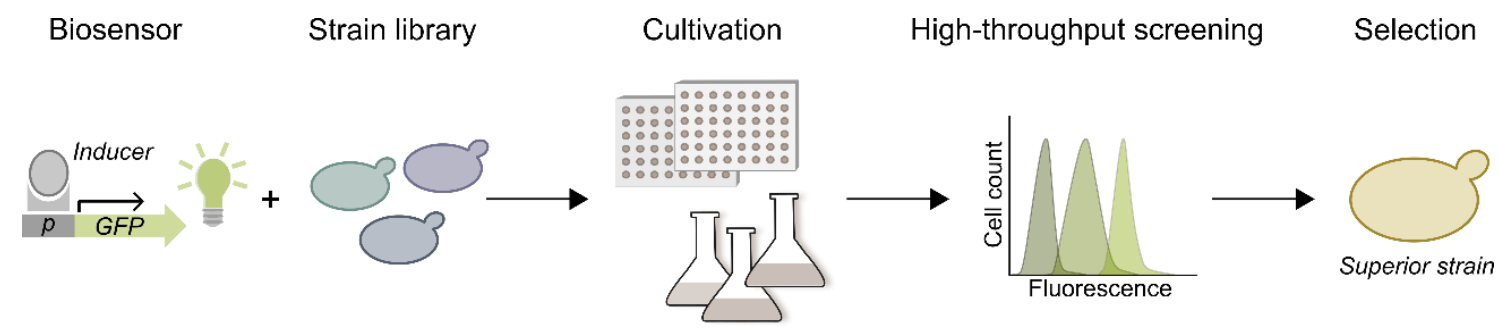

Figure 4: Application of biosensors for high-throughput screenings of strain libraries. Highthroughput screenings in combination with biosensors allow for the identification of yeast strains with superior phenotypes among millions of strain variants. A biosensor consisting of an inducible promoter (p) coupled to GFP enables the quantification of small molecules by generating an easily detectable output (here: fluorescence). To identify strains with the desired property, strain libraries are cultivated in shake flasks or multiwell plates and tested in biosensor-aided flow cytometry screens.

For an efficient screening, cultivation and detection of strains must be high throughput. Massive parallel cultivation is achieved in multiwell plate formats in small volumes, or for bigger volumes in common shake flasks. For screening, the most ubiquitous and facile method is microfluidic cell sorting, in particular fluorescence-activated cell sorting (FACS). Biosensors that couple increased production of an intracellular metabolite with expression of fluorescent proteins like GFP, enable high-throughput FACS of strain libraries (Schallmey et al., 2014; Nielsen \& Keasling, 2016). Following the sorting of highest fluorescent individual cells in a screening, chosen strains are further evaluated. The nature of the genetic element that is responsible for the desired phenotypic change, can be identified with classical molecular genetic methods. By reintroducing it into different producer strain backgrounds, positive effects can be evaluated and strain performance further optimized (Woolston et al., 2013). FACS screenings have been successfully employed recently for the identification of improved $S$. cerevisiae strains with higher xylose transportation capacity (Wang et al., 2014) and increased fatty acylCoA pools (Dabirian et al., 2019). 


\subsection{Aim of this thesis}

The aim of this thesis was to optimize octanoic acid production in S. cerevisiae through development of new strategies as well as exploitation of classical approaches, and to investigate its physiological impact on the producing strain. Previous to this work, an $S$. cerevisiae strain was engineered, which has a fatty acid production spectrum that is shifted from long-chain fatty acids to primarily octanoic acid. This was achieved by rationally engineering the native FAS enzyme, generating the modified variant $F A S^{R 1834 K}$ (Gajewski et al., 2017). This strain was to be used as a starting strain for further optimization.

To quantify octanoic acid produced by yeast, at the beginning of this thesis, it had to be extracted, methylated and analyzed via GC, which is a laborious and timeconsuming work. For this purpose, a biosensor was to be developed that comes with a linear detection range within currently produced levels and an easily quantifiable output, ideally fluorescence. This biosensor should subsequently be used in a high-throughput screening of a whole genome multicopy plasmid library to detect improved strain variants. For such a screening, the biosensor would have to be modified and verified on single cell level in a FACS setup. The highthroughput FACS screening of the strain library would allow for detection of genes that when overexpressed increase octanoic acid titers of the producer strain.

The first transcriptomics analysis during fermentation of an S. cerevisiae octanoic acid producer strain was to be conducted. Such an analysis will give profound insights into the yeast's transcriptional response to octanoic acid production. Subsequent analysis of differential gene expression in comparison to a reference strain aims for identification of promising genetic targets for engineering.

Rational approaches, i.e. knockout, overexpression or mutation of genes encoding for enzymes involved in fatty acid biosynthesis, transport or transcriptional regulation were to be evaluated. With the aim to increase strain robustness, an ALE experiment should be conducted in parallel shake flasks in the presence of increasing octanoic acid concentrations and cell growth was to be monitored over prolonged fermentation time. Toxicity of octanoic acid on S. cerevisiae strains used in industrial fermentations was to be evaluated to identify future production hosts. 


\section{General discussion}

The first goal of this thesis was to develop and apply new strategies to improve octanoic acid production in yeast. Secondly, the aim was to generate further understanding of the physiology of the yeast during octanoic acid production. In the following subchapters, the results of the attached publications and additional results section will be discussed in a wider context, i.e. how they can be expanded, and by which tools they can be complemented to guide future strain engineering efforts. Furthermore, it will be discussed which factors restrict octanoic acid production in yeast to date and how to overcome them.

\subsection{Octanoic acid biosensor and high-throughput screenings - achievements and opportunities}

This thesis contains one publication (chapter 6.1; Baumann et al., 2018) that describes the development of the first TF-based biosensor for screening of shortand medium-chain fatty acid production. Our first attempt had been to use a previously published GPCR-based octanoic acid biosensor (Mukherjee et al., 2015), improve its low linear and dynamic range and make it functional in culture broth. However, it was not possible to reproduce the published results (data not shown), which led us to develop an entirely new biosensor system for octanoic acid detection.

As depicted in chapter 2.3.4, TF-based biosensors enable facile and rapid detection of small molecules through combination with an output such as fluorescence. A biosensor based on the pPDR12 promoter was developed, which is regulated solely by the TF War1. A quantifiable output was achieved by coupling pPDR12 to GFP expression. The biosensor responded to C6, C7 and C8 fatty acids, respectively, in a concentration-dependent manner over high linear and dynamic ranges. It was able to reliably detect $0.01 \mathrm{mM}$ up to $2 \mathrm{mM}$ octanoic acid and generated an up to 10 -fold increase in fluorescence. Furthermore, this is the first biosensor that reliably quantified octanoic acid in culture supernatants of a producer strain - and is even functional in complex medium. This was confirmed by transforming a non-producer strain with a multicopy plasmid containing the biosensor and incubating this biosensor strain with culture supernatants containing 
different amounts of octanoic acid (chapter 6.1, Baumann et al., 2018).

The application of the biosensor is not restricted to screen octanoic acid producer strains. For instance, it can be applied to screen producer strains of fatty acids of shorter chain lengths (C6, C7) to which it is also responsive (Holyoak et al., 1999; Hatzixanthis et al., 2003; Baumann et al., 2018). C6 and C7 fatty acids are technologically relevant, for example as precursors for biofuels (Peralta-Yahya et al., 2012). Furthermore, the development, optimization and verification process of the biosensor can serve as a model for similar approaches for other industrially relevant compounds.

As a complimentary future strategy, it could also be of interest to screen for clones with an increased fatty acid precursor supply. Sufficient precursor supply is a common bottleneck in microbial pathways. To detect $S$. cerevisiae strains with increased malonyl-CoA levels, another established biosensor system could be utilized. It was developed based on the bacterial TF FapR-fapO system which allows for sensing of intracellular malonyl-CoA levels (Li et al., 2015; David et al., 2016). David et al. (2016) established a system in which the biosensor detects malonyl-CoA accumulation and thereupon induces expression of a heterologous pathway which uses malonyl-CoA for 3-hydroxypropionate production. Li et al. (2015) successfully adapted the FapR-fapO biosensor system for use in a FACS screening of a genetic library and detected two new targets that, when overexpressed, increased 3-hydroxypropionte levels. The application of this biosensor to screen an octanoic acid producer strain library for increased malonylCoA levels would have to be considered carefully though. It has been shown that elevated malonyl-CoA levels do not necessarily lead to higher octanoic acid titers. On the contrary, enhanced malonyl-CoA levels (generated by expression of mutated ACC1 variants) have favored chain elongation of medium-chain fatty acids (Choi \& Da Silva, 2014; Zhou et al., 2016; Besada-Lombana et al., 2017; Zhu et al., 2020), leading to an increase in long-chain fatty acids.

Despite the necessity for high-throughput screening techniques, to date, biosensors are one of the very rare devices for efficient strain analysis. Recently, an alternative method has been reported for high-throughput quantification of the fatty acid composition in yeast cells (Xue et al., 2020). It is based on the 
observation that produced fatty acids of different chain lengths are proportionally present in phosphatidylcholines of yeast membrane lipids. Matrix-assisted laser desorption/ionization time-of-flight mass spectrometry (MALDI-ToF MS) measurement of yeast colonies allowed for analysis of the fatty acid spectrum produced by individual strains within about 2 seconds per sample. Verification of the method was achieved by screening 288 colonies of a FAS2 (KS domain) mutant library combined with automated peak analysis via a Python script. The setup allowed for identification of strain variants producing medium-chain fatty acids in varying ratios (Xue et al., 2020). This method could be an interesting alternative to the use of the pPDR12-GFP biosensor to measure octanoic acid producer strains as it is comparable regarding speed, throughput and accuracy, and comes along with easy sample preparation. However, a major drawback is the need for very costly equipment. The choice of method therefore also depends on the resources and equipment at hand.

The successful development of the pPDR12-GFP octanoic acid biosensor enabled us to conduct a high-throughput screening to identify new targets that increased octanoic acid production, as described in the attached manuscript in chapter 6.4 (Baumann et al., 2020a). For use of the pPDR12-GFP biosensor in the envisioned screening, some aspects were modified. A combined biosensor and octanoic acid producer strain was constructed by genomically integrating pPDR12-GFP in a producer strain. The genomic integration of the biosensor drastically decreased the single-cell noise in comparison to expression from a multicopy plasmid. Additional knockout of FAA2 (chapter 2.3.1) and integration of a positive feedback loop ( $\triangle$ pWAR1::pPDR12) led to a strain amenable to high-throughput screening via FACS.

The FACS screening was designed to include consecutive enrichment rounds to reduce the rate of false positive strains. Eventually, the screening of a gene overexpression library resulted in the detection of two new targets, Kcs1 and Fsh2, that had never been described to be involved in fatty acid metabolism and that, in combined overexpression, led to a $55 \%$ increase in octanoic acid titers (chapter 6.4, Baumann et al., 2020a). To date, the role of the enzyme encoded by FSH2 remains obscure. However, Fsh1, which is about $25 \%$ homologous, plays a role 
in lipid metabolism, and $F S H 1$ overexpression was shown to favor the production of free fatty acids (Ramachandran et al., 2020). Therefore, it can be speculated that $F S H 2$ also plays a - yet unknown - role in lipid metabolism. KCS1 encodes an inositol pyrophosphate synthase that phosphorylates different inositol phosphates, which are important signaling molecules in the cell (Saiardi et al., 1999, 2000; Dubois et al., 2002). A recent publication showed that KCS1 knockout affects S-adenosyl-L-methionine, glycolytic intermediates' and ATP levels (Chen et al., 2021). Therefore, it is likely that KCS1 overexpression affects glycolysis, leading to increased octanoic acid titers. Further analyses, e.g. transcriptomics or proteomics analyses, with FSH2 and KCS1 overexpression strains could be conducted to unravel the exact molecular mechanisms.

The methodology of the performed high-throughput FACS screening has been developed with diverse applications in mind. Firstly, the developed method can be used to screen various genetic libraries. The library that was chosen consisted of the entire yeast genome cloned on multicopy plasmids with native promoter and terminator regions comprising of 1588 plasmids in total (Jones et al., 2008). This library enables the overexpression of genes under control of their native regulatory sequences; however, it must be considered that several genes are contained on each plasmid. To identify single genes responsible for an observed phenotype laborious cloning and testing must be conducted. To circumvent this, a library consisting of only one gene per plasmid would be advantageous. An example for this is the Molecular Barcoded Yeast Open Reading Frame (MoBY-ORF) collection which comprises 4956 genes (Ho et al., 2009). The MoBY-ORF library is composed of centromeric plasmids, each carrying a single yeast ORF flanked by endogenous promoter and terminator sequences. These sequences are flanked by unique oligonucleotide barcodes which facilitate the correlation of an interesting phenotype to the responsible gene. The usability of the MoBY-ORF library was demonstrated recently in a FadR biosensor-assisted screening for genes enhancing fatty acyl-CoA pools, eventually resulting in increased fatty alcohol levels (Dabirian et al., 2019). To identify genes, library plasmids are purified, and barcodes sequenced, which makes the use of this library very straightforward. Despite the advantages of the MoBY-ORF library, both described libraries have the same scope as they comprise the native yeast genome. 
Another interesting approach could be the screening of a library of mutated variants of pathway genes. For instance, analogous to the abovementioned MALDI/ToF MS screening of FAS2 KS domain variants by Xue et al. (2020), a library of mutated variants of FAS1 could be screened. The MPT domain of FAS1 would state a promising target for mutation as it plays an eminent role in the loading of malonylCoA elongation units as well as the release of elongated fatty acyl-CoA chains (chapter 2.2.4). Rational engineering or epPCR could be employed to generate a library of mutated FAS1-MPT domain variants. In a pPDR12-GFP biosensorassisted mutant library screening, new versions could be detected that lead to enhanced release of medium-chain acyl-CoAs. Thereby, new enzyme variants with superior properties to the already known variant $F A S 1^{1834 K}$ (Gajewski et al., 2017) could be detected.

For screening strain libraries, single cell flow cytometry in combination with cell sorting, i.e. FACS, is the predominant method of choice. This is mainly due to widespread equipment availability and rapid and ultra-high throughput measurements (Dietrich et al., 2010). However, single cell FACS screens are based on the assumption that the production capacity of a cell is proportional to the intracellular content. For compounds that are mostly released from the cell via passive diffusion, a FACS screening is likely effective. For compounds that are mostly excreted by active transport mechanisms, however, FACS may favor the selection of transport-deficient cells that retain the product intracellularly (Wagner et al., 2018). The mode of release of octanoic acid is not entirely clear yet but it is likely secreted from the cell by a mixture of passive diffusion and active transport (Legras et al., 2010; Leber \& Da Silva, 2014; Borrull et al., 2015). Preliminary FACS experiments revealed a correlation between intra- and extracellular octanoic acid amounts. It was shown that strains with higher octanoic acid titers in the supernatant (determined by GC) also showed a higher biosensor activation as measured via single cell fluorescence (Baumann et al., 2020a, chapter 6.4). Based on these observations it was concluded that a FACS screening is suitable to detect strains with increased intra- and extracellular octanoic acid titers.

Nevertheless, due to the toxicity of octanoic acid on the yeast cells (Viegas et al., 1989; Borrull et al., 2015), it is highly desirable to have cells with a high secretion 
rate. Therefore, the development of screenings that detect strains with increased octanoic acid secretion is worthwhile considering. For this aim, the extracellular amount of the compound needs to be quantified. This could be achieved, for instance, in a two-cell system with separate cultivation of the producer and biosensor strains in microtiter plates. Supernatants of the producers are added to the biosensor strain and fluorescence evaluated in e.g. multiwell plate readers. Despite a lower throughput in comparison to single cell FACS, an advantage of this method is that the limits of the linear detection range can be circumvented by diluting the samples. For a microtiter plate screening, strain cultivation, handling, and metabolite analyses need to be performed on automated robotic platforms to reach the aspired throughput. However, the lack of such facilities is often an obstacle for realizing this type of screening (Dietrich et al., 2010).

An interesting alternative that circumvents the need for these facilities, is a dropletbased flow cytometry screening. In such a two-cell system, producer and biosensor cells are encapsulated and cultivated in microdroplets, e.g. alginate capsules. The compound-of-interest is synthesized by the producer cell, secreted into the capsule, and consequently taken up by the biosensor cell (Meyer et al., 2015; Wagner et al., 2018). Meyer et al. (2015) applied this approach to identify Bacillus subtilis cells with improved vitamin B production. For the screening of the $B$. subtilis strain library, it proved to be essential to use a biosensor strain with an orthogonal nutrient requirement. This prevented competition between producer and biosensor cells for growth substrates. Furthermore, the microcapsules that contained the biosensor strain and the $B$. subtilis library, were embedded in a hydrophobic solvent to minimize crosstalk between library strains. The resulting readout, biosensor fluorescence, was evaluated via droplet FACS analysis. The advantage of this method is that it can quantify secreted molecules in very high throughput. In the framework of this project, this method was evaluated for a biosensor strain in combination with octanoic acid producer cells (data not shown). Preliminary tests revealed that the hydrophobic solvent used to embed the microcapsules did not prevent crosstalk between library strains. Due to the molecular characteristics of octanoic acid, it was impossible to generate an appropriate (hydrophobic) solvent and therefore this screening approach was not pursued further. 
Despite various challenges regarding biosensor and screening development, many successful examples - including this work - have shown the potential of the technology, which is predicted to drastically facilitate metabolic engineering in the upcoming years (Nielsen \& Keasling, 2016).

\subsection{Saccharomyces cerevisiae physiology during octanoic acid production}

One of the aims of this project was to address the lack of knowledge of the producer strain's physiology during octanoic acid production. In the attached publication Baumann et al. (2021) in chapter 6.3 this issue was addressed by conducting the first transcriptomics analysis of an octanoic acid producer strain, SHY34/fusFASRK, in comparison to a reference strain, SHY34/fusFASWT. The transcriptomic landscape was analyzed at three sampling times during octanoic acid production and compared to literature data of the transcriptomic response of wild type strains to supplemented octanoic acid. Thereby, a new target, RPL40B, plasmid-based overexpression of which led to a $40 \%$ increase in octanoic acid titers, was identified. This was the first report of an involvement of RPL4OB, which encodes for a subunit of the ribosome, in fatty acid biosynthesis (chapter 6.3, Baumann et al., 2021). It would be of great interest to investigate this further, e.g. by analyzing knockout or knockdown mutants of RPL40B or of its paralog RPL40A. To gain insight into the mechanism behind this effect, proteome analyses or ribosome profiling, so-called RiboSeq (Weinberg et al., 2016), could be performed.

Another interesting finding of the RNA-Seq experiment was that the (plasmidbased) expression of fusFASRK was much higher than of the wild type analogue fusFASWT. Chain length control of the modified fusFASRK is leaky enabling synthesis of long-chain fatty acids (Gajewski et al., 2017), which are essential for instance as components of membranes and energy supply (Klug \& Daum, 2014). Whilst the majority of fatty acyls is released from the FAS ${ }^{\mathrm{RK}}$ complex when reaching a chain length of $\mathrm{C} 8$, a fraction of the fatty acyls is extended with malonyl-CoA units to generate long-chain fatty acids. The RNA-Seq results suggest that the gene expression of SHY34/fusFASRK is probably not optimized for maximum octanoic acid production but rather the synthesis of enough long-chain fatty acids for 
survival. Another factor that limits titers is the cell's utilization of fatty acid precursors for other metabolic processes (van Rossum et al., 2016). This competition for cellular resources might explain the limit in octanoic acid production that was observed in various experiments, stressing the importance of also improving precursor supply - as reviewed in chapter 6.2 (Baumann et al., 2020b).

A crucial conclusion of the transcriptomic analysis was that increasing octanoic acid titers correlated with increased differential gene expression and therefore seemed to influence overall strain physiology. The effect of overexpression and deletion, respectively, of all genes that were up- or downregulated at more than one sampling time was evaluated. Interestingly, the majority thereof did not have any effect on octanoic acid titers or growth. The differential expression of these genes during octanoic acid production therefore seems to serve other purposes, e.g. to cope with octanoic acid toxicity. This would be in accordance with another finding of the RNA-Seq experiment, i.e. negative effects of octanoic acid on the producer cell's growth. The final OD of the producer strain remained lower than that of the reference strain. The toxicity of octanoic acid on different $S$. cerevisiae strains was also observed in separate experiments (chapter 5.1.1). Strategies that could mitigate the toxic effects will be discussed in the following subchapter.

\subsection{Strategies to increase strain robustness and reduce octanoic acid toxicity}

Increasing the robustness of $S$. cerevisiae to octanoic acid is vital for generating a high performing producer strain. The mechanisms of octanoic acid toxicity and transport are not entirely elucidated yet. Octanoic acid that is produced by the yeast cell is readily secreted, likely by a mixture of passive diffusion and active transport (Legras et al., 2010; Leber \& Da Silva, 2014; Borrull et al., 2015). At low media pH, which is the common condition in industrial processes, octanoic acid is highly toxic because it is mostly present in its undissociated form which presumably can easily enter the cell by passive diffusion. In the cytosol of the cell, it dissociates, leading to a drop in intracellular $\mathrm{pH}$ and an accumulation of toxic anions. To counteract, the cell activates plasma membrane $\mathrm{H}^{+}$-ATPases which pump out excessive protons (Viegas et al., 1998). In addition to this mechanism, octanoic acid, due to 
its hydrophobic character, incorporates into the membrane. This disturbs membrane integrity and composition and its essential function as transport system and selective barrier. Eventually these impairments lead to membrane leakage and cell death (Viegas et al., 1998; Cabral et al., 2001; Legras et al., 2010; Liu et al., 2013).

Legras et al. (2010) analyzed a wild type strain's response to octanoic acid supplementation by transcriptomics analysis and subsequent study of knockout mutants. They identified oxidative stress as well as the transporter Tpo1 and the transporter-regulating TFs War1 and Pdr3 to be minorly involved in adaptation to the acids. The main role in yeast resistance to octanoic acid was ascribed to the transporter Pdr12. In the framework of this project, different strategies were used aiming to increase yeast robustness, including rational engineering of such reported targets (chapter 5.1.3). Despite the reported decrease in octanoic acid resistance for the knockout mutants $\Delta p d r 12, \Delta t p o 1, \Delta w a r 1$ and $\Delta p d r 3$ (Legras et al., 2010), overexpression of neither of these genes increased growth of a wild type strain in the presence of octanoic acid (Figure 9). Possible reasons for this are discussed in the additional results chapter 5.1.3 in detail.

As this rational engineering strategy was unsuccessful in generating a robust strain, an ALE experiment was conducted. However, the improved phenotype of an evolved population was not reproducible from isolated single cells, as shown in the additional results section (chapter 5.1.2). The engineering of targets reported from an ALE experiment from another group (Zhu et al., 2020) did also not result in an improved phenotype in our producer strain (Figure 8). Despite ALE being a successful instrument in many cases to improve yeast strain robustness e.g. to aromatic acids at low pH (Pereira et al., 2020), acetic acids (González-Ramos et al., 2016), or high temperatures (Caspeta et al., 2014), it doesn't seem to be the ideal method to elevate yeast tolerance to high levels of octanoic acid. When performing ALE with E. coli strains with high octanoic acid concentrations, a strain with strongly increased tolerance that was able to grow in the presence of up to 30 $\mathrm{mM}$ (=4.3 g/L) octanoic acid was generated (Royce et al., 2015). It is important to mention, however, that these experiments were conducted at neutral $\mathrm{pH}$, when octanoic acid toxicity is much lower than at acidic pH (Viegas \& Sá-Correia, 1997). 
The increased tolerance of the E. coli strain was mainly attributed to changes in the bacterial membrane integrity and rigidity and an increase in the average lipid tail length (Royce et al., 2015; Chen et al., 2020). Such changes cannot be transferred one-to-one to yeast due to differences in membrane architecture and composition. There have been several attempts to engineer the yeast membrane for increasing its stress tolerance, which have recently been summarized (Qi et al., 2019). A beforementioned example (chapter 2.3.1) for this strategy is the modification of ACC1. Expression of a modified version, ACC1S1157A, was reported to result in an enhanced oleic acid content in the membrane, conferring the strain with higher robustness to octanoic acid and even leading to higher titers in a production strain (Liu et al., 2013; Besada-Lombana et al., 2017). In our experiments, however, a strain overexpressing $A C C 1^{S 1157 A}$ did not show a change of growth in the presence of octanoic acid, possibly due to masking effects of the native ACC1 gene expressed from the strain background. Despite these contradictory results, it cannot be excluded that membrane engineering could be a beneficial complimentary strategy for generating a more robust production strain.

Despite some advances, current robustness levels would be insufficient for an industrial-scale production of octanoic acid via yeast. An octanoic acid market report estimates that titers need to reach at least $60 \mathrm{~g} / \mathrm{L}$ for an economically feasible production at competitive prices (personal communication, H. Marckmann, Clariant). Considering the limited success of the above stated attempts for increasing robustness, it is unlikely that laboratory yeast strains can be generated for the required tolerance level. The primary optimization of the production strain for increasing titers should certainly be conducted in laboratory strains due to their ease in handling and genetic modification. The following steps should then be conducted in an inherently more tolerant strain. For determining the tolerance levels of different strains, toxicity tests with five $S$. cerevisiae strains in different media and growth conditions at an initial pH around 6.5 were conducted (Figure 5, Figure 6 in chapter 5.1.1). Hereby, the total inhibitory concentration was shown to differ widely between 400 and $800 \mathrm{mg} / \mathrm{L}$ octanoic acid. All four tested industrial strains were more robust than the laboratory strain CEN.PK2-1C, and even more so, when preadapted to octanoic acid. 
As the tolerance levels of the evaluated industrial strain is still not high enough, in the next step, the biosynthesis could be coupled to a product detoxification step. One possibility is an in situ product removal in a two-phase fermentation with an appropriate solvent (Henritzi et al., 2018; Zheng et al., 2020). Henritzi et al. (2018) added dodecane to an octanoic acid production strain and achieved an increase in overall titers, i.e. in the aqueous and dodecane phase, by about $40 \%$. Nevertheless, only a fraction of the produced octanoic acid was sequestered by dodecane. Hence, for an efficient in situ extraction of octanoic acid, other solvents would need to be evaluated.

An alternative strategy to detoxify octanoic acid is the transformation of octanoic acid into a non-toxic derivative. For instance, octanoic acid could be captured in the form of nonpolar storage lipids like triacylglycerols (TAGs). TAGs, which are stored in lipid droplets in the cell, can serve as a sink for free fatty acids, thereby avoiding toxic and membrane-disturbing effects (Friedlander et al., 2016). The activated form of octanoic acid, octanoyl-CoA, could be incorporated into TAGs by heterologous, medium-chain specific enzymes, which have been discovered in plants and fungi (Lardizabal et al., 2002; Aymé et al., 2015; Reynolds et al., 2019; Yuan et al., 2019). The captured fatty acids could be retrieved from TAGs by activation of lipases in a late phase of fermentation when toxic effects of free fatty acids on growth are negligible. A specific lipase or an endogenous lipase with a broad product spectrum including medium-chain acyl moieties, such as Tgl3 (Athenstaedt \& Daum, 2003; Schmidt et al., 2013), could be used for this purpose. Cloning Tgl3 under control of an inducible promotor, would enable controlled activation at a late fermentation phase, thereby releasing octanoic acid.

\subsection{Future perspectives for octanoic acid production in Saccharomyces cerevisiae}

Octanoic acid is applied in a wide range of products and its market demand has constantly increased over the last years (Yan \& Pfleger, 2020; IndexMundi, 2020). The biotechnological production in S. cerevisiae offers a promising alternative to current production methods. The basis for this approach has already been created but the strains must be further improved. 
An S. cerevisiae production strain based on the $F A S^{R K}$ variant in combination with a knockout of medium-chain specific $\beta$-oxidation ( $\triangle$ faa2) was used as a basis for initial experiments. The overexpression of the genes RPL4OB, FSH2 and KCS1 that were identified in the framework of this thesis, improved production titers, however, their exact role in fatty acid metabolism remains to be unraveled. The developed biosensor and screening strategy present a means to screen various genetic libraries for identification of additional targets which can enhance octanoic acid production. For an efficient production, an improved precursor supply and balanced cofactors are vital and can be engineered based on knowledge from previous research as summarized in the publication in chapter 6.2 (Baumann et al., 2020b). Once a well-performing laboratory strain is established, the strain development could be transferred to an inherently more tolerant strain like EthanolRed, including overexpression of the three here identified targets. In addition, it would be of great interest to evaluate yeasts like $Y$. lipolytica as host organisms (Beopoulos et al., 2009; Friedlander et al., 2016). Y. lipolytica is an oleaginous yeast that naturally accumulates large amounts of lipids and fatty acids and could therefore be an interesting host for octanoic acid production (Beopoulos et al., 2009). Irrespective of the host organism, due to the toxicity of octanoic acid, production will have to be coupled to a detoxification strategy, such as sequestration in non-toxic TAGs.

Ideally, the sugar substrate for yeast to produce octanoic acid could derive from inexpensive, renewable resources, e.g. non-food bioenergy crops or agricultural waste (Gustavsson \& Lee, 2016; D’Espaux et al., 2017). Such lignocellulosic biomass commonly contains, in addition to glucose, the pentose sugars xylose and arabinose, which cannot naturally be fermented by yeast. For an efficient utilization of all sugars, $S$. cerevisiae needs to be genetically engineered, a challenge that has been addressed extensively and with some success (Laluce et al., 2012). Despite considerable progress in this field as well as in the engineering of yeast for fatty acid production, major improvements in octanoic acid titer and yield are necessary to advance to a sustainable and competitive large-scale production. 


\section{References}

Abatemarco, J., Hill, A., \& Alper, H. S. (2013). Expanding the metabolic engineering toolbox with directed evolution. Biotechnology Journal, 8(12), 1397-1410. https://doi.org/10.1002/biot.201300021

Athenstaedt, K., \& Daum, G. (2003). YMR313c/TGL3 encodes a novel triacylglycerol lipase located in lipid particles of Saccharomyces cerevisiae. Journal of Biological Chemistry, 278(26), 23317-23323. https://doi.org/10.1074/jbc.M302577200

Aymé, L., Jolivet, P., Nicaud, J.-M., \& Chardot, T. (2015). Molecular Characterization of the Elaeis guineensis Medium-Chain Fatty Acid Diacylglycerol Acyltransferase DGAT1-1 by Heterologous Expression in Yarrowia lipolytica. PLOS ONE, 10(11), e0143113. https://doi.org/10.1371/journal.pone.0143113

Bae, Y. S., \& Rhee, M. S. (2019). Short-term antifungal treatments of caprylic acid with carvacrol or thymol induce synergistic 6-log reduction of pathogenic Candida albicans by cell membrane disruption and efflux pump inhibition. Cellular Physiology and Biochemistry, 53(2), 285-300. https://doi.org/10.33594/000000139

Baeshen, N. A., Baeshen, M. N., Sheikh, A., Bora, R. S., Ahmed, M. M. M., Ramadan, H. A. I., Saini, K. S., \& Redwan, E. M. (2014). Cell factories for insulin production. Microbial Cell Factories, 13(141). https://doi.org/10.1186/s12934-014-0141-0

Baumann, L., Doughty, T., Siewers, V., Nielsen, J., Boles, E., \& Oreb, M. (2021). Transcriptomic response of Saccharomyces cerevisiae to octanoic acid production. FEMS Yeast Research (Accepted).

Baumann, L., Bruder, S., Kabisch, J., Boles, E., \& Oreb, M. (2020a). High-throughput screening of an octanoic acid producer strain library enables detection of new targets for increasing titers in Saccharomyces cerevisiae. Submitted.

Baumann, L., Wernig, F., Born, S., \& Oreb, M. (2020b). Engineering Saccharomyces cerevisiae for production of fatty acids and their derivatives. In J. P. Benz \& K. Schipper (Eds.), The Mycota Vol. II: Genetics and Biotechnology, 3rd edition (pp. 339-368). Springer, Switzerland.

Baumann, L., Rajkumar, A. S., Morrissey, J. P., Boles, E., \& Oreb, M. (2018). A yeast-based biosensor for screening of short- and medium-chain fatty acid production. ACS Synthetic Biology, 7(11), 2640-2646. https://doi.org/10.1021/acssynbio.8b00309

Beopoulos, A., Cescut, J., Haddouche, R., Uribelarrea, J.-L., Molina-Jouve, C., \& Nicaud, J.-M. (2009). Yarrowia lipolytica as a model for bio-oil production. Progress in Lipid Research, 48(6), 375-387. https://doi.org/10.1016/J.PLIPRES.2009.08.005

Berger, K. G. (2003). Palm kernel oil. In Encyclopedia of Food Sciences and Nutrition (2nd ed., pp. 4322-4324). Elsevier. https://doi.org/10.1016/b0-12-227055-x/01379-1

Besada-Lombana, P. B., Fernandez-Moya, R., Fenster, J., \& Da Silva, N. A. (2017). Engineering Saccharomyces cerevisiae fatty acid composition for increased tolerance to octanoic acid. Biotechnology and Bioengineering, 114(7), 1531-1538. https://doi.org/10.1002/bit.26288

Borrull, A., López-Martínez, G., Poblet, M., Cordero-Otero, R., \& Rozès, N. (2015). New insights into the toxicity mechanism of octanoic and decanoic acids on Saccharomyces cerevisiae. Yeast, 32(5), 451-460. https://doi.org/10.1002/yea.3071

Brautaset, T., \& Ellingsen, T. E. (2011). Lysine: Industrial Uses and Production. In Comprehensive Biotechnology (2nd ed., Vol. 3). Elsevier Inc. https://doi.org/10.1016/B978-0-08-0885049.00220-8

Burgard, A., Burk, M. J., Osterhout, R., Van Dien, S., \& Yim, H. (2016). Development of a commercial scale process for production of 1,4-butanediol from sugar. Current Opinion in Biotechnology, 42, 118-125. https://doi.org/10.1016/j.copbio.2016.04.016

Cabral, M. G., Viegas, C. A., \& Sá-Correia, I. (2001). Mechanisms underlying the acquisition of resistance to octanoic-acid-induced-death following exposure of Saccharomyces cerevisiae to mild stress imposed by octanoic acid or ethanol. Archives of Microbiology, 175(4), 301307. http://www.ncbi.nlm.nih.gov/pubmed/11382226 
Caspeta, L., Chen, Y., Ghiaci, P., Feizi, A., Buskov, S., Hallström, B. M., Petranovic, D., \& Nielsen, J. (2014). Altered sterol composition renders yeast thermotolerant. Science, 346(6205), 7578.

http://science.sciencemag.org/content/sci/suppl/2014/10/01/346.6205.75.DC1/Caspeta.SM. pdf

Cernak, P., Estrela, R., Poddar, S., Skerker, J. M., Cheng, Y.-F., Carlson, A. K., Chen, B., Glynn, V. M., Furlan, M., Ryan, O. W., et al. (2018). Engineering Kluyveromyces marxianus as a Robust Synthetic Biology Platform Host. MBio, 9(5), 1-16. https://mbio.asm.org/content/9/5/e01410-18

Chen, H., Zhu, N., Wang, Y., Gao, X., Song, Y., Zheng, J., Peng, J., \& Zhang, X. (2021). Increasing glycolysis by deletion of $k c s 1$ and arg82 improved S-adenosyl-I-methionine production in Saccharomyces cerevisiae. AMB Express, 11(1), 20. https://doi.org/10.1186/s13568-02101179-8

Chen, Y., Boggess, E. E., Ocasio, E. R., Warner, A., Kerns, L., Drapal, V., Gossling, C., Ross, W., Gourse, R. L., Shao, Z., et al. (2020). Reverse engineering of fatty acid-tolerant Escherichia coli identifies design strategies for robust microbial cell factories. Metabolic Engineering, 61, 120-130. https://doi.org/10.1016/j.ymben.2020.05.001

Chirala, S. S., Zhong, Q., Huang, W., \& Al-feel, W. (1994). Analysis of FAS3/ACC regulatory region

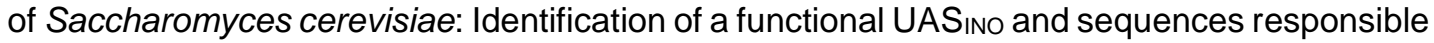
for fatty acid mediated repression. Nucleic Acids Research, 22(3), 412-418. https://doi.org/10.1093/nar/22.3.412

Choi, J. W., \& Da Silva, N. A. (2014). Improving polyketide and fatty acid synthesis by engineering of the yeast acetyl-CoA carboxylase. Journal of Biotechnology, 187, 56-59. https://doi.org/10.1016/j.jbiotec.2014.07.430

Claus, S., Jezierska, S., \& Van Bogaert, I. N. A. (2019). Protein-facilitated transport of hydrophobic molecules across the yeast plasma membrane. FEBS Letters, 593(13), 1508-1527. https://doi.org/10.1002/1873-3468.13469

D’Ambrosio, V., \& Jensen, M. K. (2017). Lighting up yeast cell factories by transcription factorbased biosensors. FEMS Yeast Research, 17(7), 103-107. https://doi.org/10.1093/femsyr/fox076

D’Espaux, L., Ghosh, A., Runguphan, W., Wehrs, M., Xu, F., Konzock, O., Dev, I., Nhan, M., Gin, J., Reider Apel, A., et al. (2017). Engineering high-level production of fatty alcohols by Saccharomyces cerevisiae from lignocellulosic feedstocks. Metabolic Engineering, 42, 115125. https://doi.org/10.1016/j.ymben.2017.06.004

Da Silva, N. A., \& Srikrishnan, S. (2012). Introduction and expression of genes for metabolic engineering applications in Saccharomyces cerevisiae. FEMS Yeast Research, 12(2), 197214. https://doi.org/10.1111/j.1567-1364.2011.00769.x

Dabirian, Y., Gonçalves Teixeira, P., Nielsen, J., Siewers, V., \& David, F. (2019). FadR-Based Biosensor-Assisted Screening for Genes Enhancing Fatty Acyl-CoA Pools in Saccharomyces cerevisiae. ACS Synthetic Biology, 8(8), https://doi.org/10.1021/acssynbio.9b00118

Dahlin, J., Holkenbrink, C., Marella, E. R., Wang, G., Liebal, U., Lieven, C., Weber, D., McCloskey, D., Ebert, B. E., Herrgård, M. J., et al. (2019). Multi-omics analysis of fatty alcohol production in engineered yeasts Saccharomyces cerevisiae and Yarrowia lipolytica. Frontiers in Genetics, 10(747). https://doi.org/10.3389/fgene.2019.00747

David, F., Nielsen, J., \& Siewers, V. (2016). Flux Control at the Malonyl-CoA Node through Hierarchical Dynamic Pathway Regulation in Saccharomyces cerevisiae. ACS Synthetic Biology, 5, 224-233. https://doi.org/10.1021/acssynbio.5b00161

Davis López, S. A., Griffith, D. A., Choi, B., Cate, J. H. D., \& Tullman-Ercek, D. (2018). Evolutionary engineering improves tolerance for medium-chain alcohols in Saccharomyces cerevisiae. Biotechnology for Biofuels, 11(1), 1-15. https://doi.org/10.1186/s13068-018-1089-9

Deen, A., Visvanathan, R., Wickramarachchi, D., Marikkar, N., Nammi, S., Jayawardana, B. C., \& Liyanage, R. (2020). Chemical composition and health benefits of coconut oil: an overview. 
Journal of the Science of Food and Agriculture. https://doi.org/10.1002/jsfa.10870

Dietrich, J. A., McKee, A. E., \& Keasling, J. D. (2010). High-throughput metabolic engineering: Advances in small-molecule screening and selection. Annual Review of Biochemistry, 79(1), 563-590. https://doi.org/10.1146/annurev-biochem-062608-095938

Dragosits, M., \& Mattanovich, D. (2013). Adaptive laboratory evolution - principles and applications for biotechnology. Microbial Cell Factories, 12(1), 64. https://doi.org/10.1186/1475-2859-1264

Dubois, E., Scherens, B., Vierendeels, F., Ho, M. M. W., Messenguy, F., \& Shears, S. B. (2002). In Saccharomyces cerevisiae, the inositol polyphosphate kinase activity of Kcs $1 p$ is required for resistance to salt stress, cell wall integrity, and vacuolar morphogenesis. Journal of Biological Chemistry, 277(26), 23755-23763. https://doi.org/10.1074/jbc.M202206200

Fargione, J., Hill, J., Tilman, D., Polasky, S., \& Hawthorne, P. (2008). Land clearing and the biofuel carbon debt. Science, 319(5867), 1235-1238. https://doi.org/10.1126/science.1152747

Fernandez-Moya, R., Leber, C., Cardenas, J., \& Da Silva, N. A. (2015). Functional replacement of the Saccharomyces cerevisiae fatty acid synthase with a bacterial type II system allows flexible product profiles. Biotechnology and Bioengineering, 112(12), 2618-2623. https://doi.org/10.1002/bit.25679

Friedlander, J., Tsakraklides, V., Kamineni, A., Greenhagen, E. H., Consiglio, A. L., MacEwen, K., Crabtree, D. V., Afshar, J., Nugent, R. L., Hamilton, M. A., et al. (2016). Engineering of a high lipid producing Yarrowia lipolytica strain. Biotechnology for Biofuels, 9(1), 77. https://doi.org/10.1186/s13068-016-0492-3

Gajewski, J., Pavlovic, R., Fischer, M., Boles, E., \& Grininger, M. (2017). Engineering fungal de novo fatty acid synthesis for short chain fatty acid production. Nature Communications, 8 , 14650. https://doi.org/10.1038/ncomms 14650

Generoso, W. C., Gottardi, M., Oreb, M., \& Boles, E. (2016). Simplified CRISPR-Cas genome editing for Saccharomyces cerevisiae. Journal of Microbiological Methods, 127, 203-205. https://doi.org/10.1016/J.MIMET.2016.06.020

Gibson, D. G., Glass, J. I., Lartigue, C., Noskov, V. N., Chuang, R. Y., Algire, M. A., Benders, G. A., Montague, M. G., Ma, L., Moodie, M. M., et al. (2010). Creation of a bacterial cell controlled by a chemically synthesized genome. Science, 329(5987), 52-56. https://doi.org/10.1126/science.1190719

Gibson, D. G., Young, L., Chuang, R. Y., Venter, J. C., Hutchison, C. A., \& Smith, H. O. (2009). Enzymatic assembly of DNA molecules up to several hundred kilobases. Nature Methods, 6(5), 343-345. https://doi.org/10.1038/nmeth.1318

Gietz, R. D., \& Schiestl, R. H. (2007). High-efficiency yeast transformation using the LiAc/SS carrier DNA/PEG method. Nature Protocols, 2(1), 31-34. https://doi.org/10.1038/nprot.2007.13

Goffeau, A., Barrell, G., Bussey, H., Davis, R. W., Dujon, B., Feldmann, H., Galibert, F., Hoheisel, J. D., Jacq, C., Johnston, M., et al. (1996). Life with 6000 genes. Science, 274(5287), 546567. https://doi.org/10.1126/science.274.5287.546

González-Ramos, D., Gorter de Vries, A. R., Grijseels, S. S., van Berkum, M. C., Swinnen, S., van den Broek, M., Nevoigt, E., Daran, J.-M. G., Pronk, J. T., \& van Maris, A. J. A. (2016). A new laboratory evolution approach to select for constitutive acetic acid tolerance in Saccharomyces cerevisiae and identification of causal mutations. Biotechnology for Biofuels, 9, 173. https://doi.org/10.1186/s13068-016-0583-1

González-Ramos, D., van den Broek, M., van Maris, A. J., Pronk, J. T., \& Daran, J.-M. G. (2013). Genome-scale analyses of butanol tolerance in Saccharomyces cerevisiae reveal an essential role of protein degradation. Biotechnology for Biofuels, 6, 48. https://doi.org/10.1186/17546834-6-48

Gustavsson, M., \& Lee, S. Y. (2016). Prospects of microbial cell factories developed through systems metabolic engineering. Microbial Biotechnology, 9(5), 610-617. https://doi.org/10.1111/1751-7915.12385

Hasslacher, M., Ivessa, A. S., Paltauf, F., \& Kohlwein, S. D. (1993). Acetyl-CoA carboxylase from 
yeast is an essential enzyme and is regulated by factors that control phospholipid metabolism. Journal of Biological Chemistry, 268(15), 10946-10952. https://pubmed.ncbi.nlm.nih.gov/8098706/

Hatzixanthis, K., Mollapour, M., Seymour, I., Bauer, B. E., Krapf, G., Schüller, C., Kuchler, K., \& Piper, P. W. (2003). Moderately lipophilic carboxylate compounds are the selective inducers of the Saccharomyces cerevisiae Pdr12p ATP-binding cassette transporter. Yeast, 20(7), 575-585. https://doi.org/10.1002/yea.981

Heil, C. S., Wehrheim, S. S., Paithankar, K. S., \& Grininger, M. (2019). Fatty Acid Biosynthesis: Chain-Length Regulation and Control. ChemBioChem, 20(18), 2298-2321. https://doi.org/10.1002/cbic.201800809

Henritzi, S., Fischer, M., Grininger, M., Oreb, M., \& Boles, E. (2018). An engineered fatty acid synthase combined with a carboxylic acid reductase enables de novo production of 1-octanol in Saccharomyces cerevisiae. Biotechnology for Biofuels, 11(150). https://doi.org/10.1186/s13068-018-1149-1

Hettema, E. H., van Roermund, C. W., Distel, B., van den Berg, M., Vilela, C., Rodrigues-Pousada, C., Wanders, R. J., \& Tabak, H. F. (1996). The ABC transporter proteins Pat1 and Pat2 are required for import of long-chain fatty acids into peroxisomes of Saccharomyces cerevisiae. The EMBO Journal, 15(15), 3813-3822. https://doi.org/10.1002/j.1460-2075.1996.tb00755.x

Higgins, C. F. (1994). Flip-flop: The transmembrane translocation of lipids. Cell, 79(3), 393-395. https://doi.org/10.1016/0092-8674(94)90248-8

Hiltunen, J. K., Mursula, A. M., Rottensteiner, H., Wierenga, R. K., Kastaniotis, A. J., \& Gurvitz, A. (2003). The biochemistry of peroxisomal $\beta$-oxidation in the yeast Saccharomyces cerevisiae. FEMS Microbiology Reviews, 27(1), 35-64. https://doi.org/10.1016/S0168-6445(03)00017-2

Ho, C. H., Magtanong, L., Barker, S. L., Gresham, D., Nishimura, S., Natarajan, P., Koh, J. L. Y., Porter, J., Gray, C. A., Andersen, R. J., et al. (2009). A molecular barcoded yeast ORF library enables mode-of-action analysis of bioactive compounds. Nature Biotechnology, 27(4), 369377. https://doi.org/10.1038/nbt.1534

Holyoak, C. D., Bracey, D., Piper, P. W., Kuchler, K., \& Coote, P. J. (1999). The Saccharomyces cerevisiae weak-acid-inducible $\mathrm{ABC}$ transporter Pdr12 transports fluorescein and preservative anions from the cytosol by an energy-dependent mechanism. Journal of Bacteriology, 181(15), 4644-4652. http://www.ncbi.nlm.nih.gov/pubmed/10419965

Hong, K. K., \& Nielsen, J. (2012). Metabolic engineering of Saccharomyces cerevisiae: A key cell factory platform for future biorefineries. Cellular and Molecular Life Sciences, 69(16), 26712690. https://doi.org/10.1007/s00018-012-0945-1

IndexMundi. (2020). Coconut Oil Production by Country in 1000 MT; Palm Kernel Oil Production by Country in $1000 \mathrm{MT}$. https://www.indexmundi.com/agriculture/?commodity=coconutoil\&graph=production

Jinek, M., Chylinski, K., Fonfara, I., Hauer, M., Doudna, J. A., \& Charpentier, E. (2012). A programmable dual-RNA-guided DNA endonuclease in adaptive bacterial immunity. Science, 337(6096), 816-821. https://doi.org/10.1126/science.1225829

Jones, G. M., Stalker, J., Humphray, S., West, A., Cox, T., Rogers, J., Dunham, I., \& Prelich, G. (2008). A systematic library for comprehensive overexpression screens in Saccharomyces cerevisiae. Nature Methods, 5(3), 239-241. https://doi.org/10.1038/nmeth.1181

Kaplan, W., Abhishek, S., Kolaczyk, E., \& Shappell, H. (2016). Insulin trade profile. Health Action International, 1-42. https://haiweb.org/wp-content/uploads/2016/04/ACCISS-PatentReportFINAL.pdf

Klug, L., \& Daum, G. (2014). Yeast lipid metabolism at a glance. FEMS Yeast Research, 14(3), 369-388. https://doi.org/10.1111/1567-1364.12141

Knoll, L. J., Johnson, D. R., \& Gordon, J. I. (1994). Biochemical Studies of Three Saccharomyces cerevisiae Acyl-CoA Synthetases, Faalp, FaaZp, and Faa3p*. The Journal of Biological Chemistry, 269(23), 16348-16356. http://www.jbc.org/content/269/23/16348.full.pdf

Kren, A., Mamnun, Y. M., Bauer, B. E., Schüller, C., Wolfger, H., Hatzixanthis, K., Mollapour, M., 
Gregori, C., Piper, P., \& Kuchler, K. (2003). War1p, a novel transcription factor controlling weak acid stress response in yeast. Molecular and Cellular Biology, 23(5), 1775-1785. https://doi.org/10.1128/mcb.23.5.1775-1785.2003

Laluce, C., Schenberg, A. C. G., Gallardo, J. C. M., Coradello, L. F. C., \& Pombeiro-Sponchiado, S. R. (2012). Advances and developments in strategies to improve strains of Saccharomyces cerevisiae and processes to obtain the lignocellulosic ethanol - A review. Applied Biochemistry and Biotechnology, 166(8), 1908-1926. https://doi.org/10.1007/s12010-012-9619-6

Lardizabal, K. D., Mai, J. T., Wagner, N. W., Wyrick, A., Voelker, T., \& Hawkins, D. J. (2002). DGAT2 Is a New Diacylglycerol Acyltransferase Gene Family. Journal of Biological Chemistry, 276(42), 38862-38869. https://doi.org/10.1074/jbc.m106168200

Leber, C., Choi, J. W., Polson, B., \& Da Silva, N. A. (2016). Disrupted short chain specific $\beta$ oxidation and improved synthase expression increase synthesis of short chain fatty acids in Saccharomyces cerevisiae. Biotechnology and Bioengineering, 113(4), 895-900. https://doi.org/10.1002/bit.25839

Leber, C., \& Da Silva, N. A. (2014). Engineering of Saccharomyces cerevisiae for the synthesis of short chain fatty acids. Biotechnology and Bioengineering, 111(2), 347-358. https://doi.org/10.1002/bit.25021

Lee, M. E., DeLoache, W. C., Cervantes, B., \& Dueber, J. E. (2015). A Highly Characterized Yeast Toolkit for Modular, Multipart Assembly. ACS Synthetic Biology, 4(9), 975-986. https://doi.org/10.1021/sb500366v

Legras, J. L., Erny, C., Le Jeune, C., Lollier, M., Adolphe, Y., Demuyter, C., Delobel, P., Blondin, B., \& Karst, F. (2010). Activation of two different resistance mechanisms in Saccharomyces cerevisiae upon exposure to octanoic and decanoic acids. Applied and Environmental Microbiology, 76(22), 7526-7535. https://doi.org/10.1128/AEM.01280-10

Li, S., Si, T., Wang, M., \& Zhao, H. (2015). Development of a Synthetic Malonyl-CoA Sensor in Saccharomyces cerevisiae for Intracellular Metabolite Monitoring and Genetic Screening. ACS Synthetic Biology, 4(12), 1308-1315. https://doi.org/10.1021/acssynbio.5b00069

Liu, P., Chernyshov, A., Najdi, T., Fu, Y., Dickerson, J., Sandmeyer, S., \& Jarboe, L. (2013). Membrane stress caused by octanoic acid in Saccharomyces cerevisiae. Applied Microbiology and Biotechnology, 97(7), 3239-3251. https://doi.org/10.1007/s00253-0134773-5

Lowell, S. Y., Kelley, R. T., Monahan, M., Hosbach-Cannon, C. J., Colton, R. H., \& Mihaila, D. (2019). The Effect of Octanoic Acid on Essential Voice Tremor: A Double-Blind, PlaceboControlled Study. Laryngoscope, 129(8), 1882-1890. https://doi.org/10.1002/lary.27695

Mans, R., Daran, J.-M. G., \& Pronk, J. T. (2018). Under pressure: evolutionary engineering of yeast strains for improved performance in fuels and chemicals production. Current Opinion in Biotechnology, 50, 47-56. https://doi.org/10.1016/J.COPBIO.2017.10.011

Markussen, J., Damgaard, U., Diers, I., Pill, N., Hansen, M. T., Larsen, P., Norris, F., Norris, K., Schou, O., Snel, L., et al. (1986). Biosynthesis of human insulin in yeast via single-chain precursors. In D. Theodoropoulos (Ed.), Peptides (2nd ed., pp. 189-194). De Gruyter, Berlin. https://doi.org/10.1515/9783110864243-041

McGettigan, P. A. (2013). Transcriptomics in the RNA-seq era. In Current Opinion in Chemical Biology (Vol. 17, Issue 1, pp. 4-11). Elsevier Current Trends. https://doi.org/10.1016/j.cbpa.2012.12.008

Meyer, A., Pellaux, R., Potot, S., Becker, K., Hohmann, H. P., Panke, S., \& Held, M. (2015). Optimization of a whole-cell biocatalyst by employing genetically encoded product sensors inside nanolitre reactors. Nature Chemistry, 7(8), 673-678. https://doi.org/10.1038/nchem.2301

Michener, J. K., Thodey, K., Liang, J. C., \& Smolke, C. D. (2012). Applications of geneticallyencoded biosensors for the construction and control of biosynthetic pathways. Metabolic Engineering, 14(3), 212-222. https://doi.org/10.1016/j.ymben.2011.09.004

Mohd Azhar, S. H., Abdulla, R., Jambo, S. A., Marbawi, H., Gansau, J. A., Mohd Faik, A. A., \& 
Rodrigues, K. F. (2017). Yeasts in sustainable bioethanol production: A review. Biochemistry and Biophysics Reports, 10, 52-61. https://doi.org/10.1016/j.bbrep.2017.03.003

Mukherjee, K., Bhattacharyya, S., \& Peralta-Yahya, P. (2015). GPCR-Based Chemical Biosensors for Medium-Chain Fatty Acids. ACS Synthetic Biology, 4(12), 1261-1269. https://doi.org/10.1021/sb500365m

Nielsen, J., \& Keasling, J. D. (2016). Engineering Cellular Metabolism. Cell, 164(6), 1185-1197. https://doi.org/10.1016/j.cell.2016.02.004

Oh, C. S., Toke, D. A., Mandala, S., \& Martin, C. E. (1997). ELO2 and ELO3, homologues of the Saccharomyces cerevisiae ELO1 gene, function in fatty acid elongation and are required for sphingolipid formation. Journal of Biological Chemistry, 272(28), 17376-17384. https://doi.org/10.1074/jbc.272.28.17376

Peralta-Yahya, P. P., Zhang, F., del Cardayre, S. B., \& Keasling, J. D. (2012). Microbial engineering for the production of advanced biofuels. Nature, 488(7411), 320-328. https://doi.org/10.1038/nature11478

Pereira, R., Mohamed, E. T., Radi, M. S., Herrgård, M. J., Feist, A. M., Nielsen, J., \& Chen, Y. (2020). Elucidating aromatic acid tolerance at low pH in Saccharomyces cerevisiae using adaptive laboratory evolution. Proceedings of the National Academy of Sciences of the United States of America, 117(45), 27954-27961. https://doi.org/10.1073/pnas.2013044117

Pfleger, B. F., Gossing, M., \& Nielsen, J. (2015). Metabolic engineering strategies for microbial synthesis of oleochemicals. Metabolic Engineering, 29, 1-11. https://doi.org/10.1016/j.ymben.2015.01.009

Postma, E. D., Dashko, S., van Breemen, L., Taylor Parkins, S. K., van den Broek, M., Daran, J.M., \& Daran-Lapujade, P. (2021). A supernumerary designer chromosome for modular in vivo pathway assembly in Saccharomyces cerevisiae. Nucleic Acids Research, 1. https://doi.org/10.1093/nar/gkaa1167

Pronk, J. T., Steensma, H. Y., \& Van Dijken, J. P. (1996). Pyruvate metabolism in Saccharomyces cerevisiae. Yeast, 12(16), 1607-1633. https://doi.org/10.1002/(SICl)10970061(199612)12:16<1607::AID-YEA70>3.0.CO;2-4

PubChem. (2020). Compound Summary for CID 379, Octanoic acid. Information, National Center for Biotechnology. https://pubchem.ncbi.nlm.nih.gov/compound/Octanoic-acid

Qi, Y., Liu, H., Chen, X., \& Liu, L. (2019). Engineering microbial membranes to increase stress tolerance of industrial strains. Metabolic Engineering, 53, 24-34. https://doi.org/10.1016/j.ymben.2018.12.010

Ramachandran, G., Chidambaram, R., \& Nachiappan, V. (2020). FSH1 encodes lysophospholipase activity in Saccharomyces cerevisiae. Biotechnology Letters, 1-8. https://doi.org/10.1007/s10529-020-03004-x

Reynolds, K. B., Cullerne, D. P., El Tahchy, A., Rolland, V., Blanchard, C. L., Wood, C. C., Singh, S. P., \& Petrie, J. R. (2019). Identification of Genes Involved in Lipid Biosynthesis through de novo Transcriptome Assembly from Cocos nucifera Developing Endosperm. Plant \& Cell Physiology, 60(5), 945-960. https://doi.org/10.1093/pcp/pcy247

Roggenkamp, R., Numa, S., \& Schweizer, E. (1980). Fatty acid-requiring mutant of Saccharomyces cerevisiae defective in acetyl-CoA carboxylase. Proceedings of the National Academy of Sciences of the United States of America, 77(4 I), 1814-1817. https://doi.org/10.1073/pnas.77.4.1814

Royce, L. A., Yoon, J. M., Chen, Y., Rickenbach, E., Shanks, J. V., \& Jarboe, L. R. (2015). Evolution for exogenous octanoic acid tolerance improves carboxylic acid production and membrane integrity. Metabolic Engineering, 29, 180-188. https://doi.org/10.1016/j.ymben.2015.03.014

Ruffing, A. M. (2013). RNA-Seq analysis and targeted mutagenesis for improved free fatty acid production in an engineered cyanobacterium. Biotechnology for Biofuels, 6(1), 113. https://doi.org/10.1186/1754-6834-6-113

Rupilius, W., \& Ahmad, S. (2007). Palm oil and palm kernel oil as raw materials for basic oleochemicals and biodiesel. European Journal of Lipid Science and Technology, 109(4), 
433-439. https://doi.org/10.1002/ejlt.200600291

Saeedi, P., Petersohn, I., Salpea, P., Malanda, B., Karuranga, S., Unwin, N., Colagiuri, S., Guariguata, L., Motala, A. A., Ogurtsova, K., et al. (2019). Global and regional diabetes prevalence estimates for 2019 and projections for 2030 and 2045: Results from the International Diabetes Federation Diabetes Atlas, 9th edition. Diabetes Research and Clinical Practice, 157. https://doi.org/10.1016/j.diabres.2019.107843

Saiardi, A., Caffrey, J. J., Snyder, S. H., \& Shears, S. B. (2000). The inositol hexakisphosphate kinase family. Catalytic flexibility and function in yeast vacuole biogenesis. Journal of Biological Chemistry, 275(32), 24686-24692. https://doi.org/10.1074/jbc.M002750200

Saiardi, A., Erdjument-Bromage, H., Snowman, A. M., Tempst, P., \& Snyder, S. H. (1999). Synthesis of diphosphoinositol pentakisphosphate by a newly identified family of higher inositol polyphosphate kinases. Current Biology, 9(22), 1323-1326. https://doi.org/10.1016/S0960-9822(00)80055-X

Saling, P. (2005). Eco-Efficiency Analysis of biotechnological processes. Applied Microbiology and Biotechnology, 68(1), 1-8. https://doi.org/10.1007/s00253-005-1951-0

Sambrook, J., Fritsch, E., \& Maniatis, T. (1989). Molecular cloning: A laboratory manual. Second edition. In G. A. Evans (Ed.), Cold Spring Harbor Laboratory Press, New York. (Vol. 61, Issue 1). https://doi.org/10.1016/0092-8674(90)90210-6

Schallmey, M., Frunzke, J., \& Eggeling, L. (2014). Looking for the pick of the bunch: high-throughput screening of producing microorganisms with biosensors. Current Opinion in Biotechnology, 26, 148-154. https://doi.org/10.1016/J.COPBIO.2014.01.005

Scharnewski, M., Pongdontri, P., Mora, G., Hoppert, M., \& Fulda, M. (2008). Mutants of Saccharomyces cerevisiae deficient in acyl-CoA synthetases secrete fatty acids due to interrupted fatty acid recycling. FEBS Journal, 275(11), 2765-2778. https://doi.org/10.1111/j.1742-4658.2008.06417.x

Schmelzer, B., Altvater, M., Gasser, B., Sauer, M., \& Mattanovich, D. (2020). Yeast cell factories. In J. P. Benz \& K. Schipper (Eds.), The Mycota Vol. II: Genetics and Biotechnology, 3rd edition (pp. 319-337). Springer, Switzerland.

Schmidt, C., Athenstaedt, K., Koch, B., Ploier, B., \& Daum, G. (2013). Regulation of the Yeast Triacylglycerol Lipase Tgl3p by Formation of Nonpolar Lipids. Journal of Biological Chemistry, 288(27), 19939-19948. https://doi.org/10.1074/jbc.M113.459610

Schmidt, J. H. (2015). Life cycle assessment of five vegetable oils. Journal of Cleaner Production, 87(C), 130-138. https://doi.org/10.1016/j.jclepro.2014.10.011

Schüller, C., Mamnun, Y. M., Wolfger, H., Rockwell, N., Thorner, J., \& Kuchler, K. (2007). Membrane-active compounds activate the transcription factors Pdr1 and Pdr3 connecting pleiotropic drug resistance and membrane lipid homeostasis in Saccharomyces cerevisiae. Molecular Biology of the Cell, 18(12), 4932-4944. https://doi.org/10.1091/mbc.E07-06-0610

Shi, S., Chen, Y., Siewers, V., \& Nielsen, J. (2014). Improving production of malonyl coenzyme Aderived metabolites by abolishing Snf1-dependent regulation of Acc1. MBio, 5(3), e01130-14. https://doi.org/10.1128/mBio.01130-14

Steensels, J., Snoek, T., Meersman, E., Nicolino, M. P., Voordeckers, K., \& Verstrepen, K. J. (2014). Improving industrial yeast strains: Exploiting natural and artificial diversity. FEMS Microbiology Reviews, 38(5), 947-995. https://doi.org/10.1111/1574-6976.12073

Stukey, J. E., McDonough, V. M., \& Martin, C. E. (1989). Isolation and characterization of OLE1, a gene affecting fatty acid desaturation from Saccharomyces cerevisiae. Journal of Biological Chemistry, 264(28), 16537-16544. https://pubmed.ncbi.nlm.nih.gov/2674136/

Stukey, J. E., McDonough, V. M., \& Martin, C. E. (1990). The OLE1 gene of Saccharomyces cerevisiae encodes the $\Delta 9$ fatty acid desaturase and can be functionally replaced by the rat stearoyl-CoA desaturase gene. Journal of Biological Chemistry, 265(33), 20144-20149. https://doi.org/10.1016/S0021-9258(17)30481-7

Tarling, E. J., Vallim, T. Q. d. A., \& Edwards, P. A. (2013). Role of ABC transporters in lipid transport and human disease. Trends in Endocrinology and Metabolism, 24(7), 342-350. 
https://doi.org/10.1016/j.tem.2013.01.006

Taxis, C., \& Knop, M. (2006). System of centromeric, episomal, and integrative vectors based on drug resistance markers for Saccharomyces cerevisiae. BioTechniques, 40(1), 73-78. https://doi.org/10.2144/000112040

Tehlivets, O., Scheuringer, K., \& Kohlwein, S. D. (2007). Fatty acid synthesis and elongation in yeast. Biochimica et Biophysica Acta - Molecular and Cell Biology of Lipids, 1771(3), 255270. https://doi.org/10.1016/j.bbalip.2006.07.004

Teo, W. S., Hee, K. S., \& Chang, M. W. (2013). Bacterial FadR and synthetic promoters function as modular fatty acid sensor- regulators in Saccharomyces cerevisiae. Engineering in Life Sciences, 13(5), 456-463. https://doi.org/10.1002/elsc.201200113

Thakur, J. K., Arthanari, H., Yang, F., Pan, S.-J., Fan, X., Breger, J., Frueh, D. P., Gulshan, K., Li, D. K., Mylonakis, E., et al. (2008). A nuclear receptor-like pathway regulating multidrug resistance in fungi. Nature, 452(7187), 604-609. https://doi.org/10.1038/nature06836

Thim, L., Hansen, M. T., Norris, K., Hoegh, I., Boel, E., Forstrom, J., Ammerer, G., \& Fiil, N. P. (1986). Secretion and processing of insulin precursors in yeast. Proceedings of the National Academy of Sciences of the United States of America, 83(18), 6766-6770. https://doi.org/10.1073/pnas.83.18.6766

Thorwall, S., Schwartz, C., Chartron, J. W., \& Wheeldon, I. (2020). Stress-tolerant non-conventional microbes enable next-generation chemical biosynthesis. Nature Chemical Biology, 16(2), 113-121. https://doi.org/10.1038/s41589-019-0452-x

Toke, D. A., \& Martin, C. E. (1996). Isolation and characterization of a gene affecting fatty acid elongation in Saccharomyces cerevisiae. Journal of Biological Chemistry, 271(31), 1841318422. https://doi.org/10.1074/jbc.271.31.18413

Tuller, G., Nemec, T., Hrastnik, C., \& Daum, G. (1999). Lipid composition of subcellular membranes of an FY1679-derived haploid yeast wild-type strain grown on different carbon sources. Yeast, 15(14), 1555-1564. https://doi.org/10.1002/(SICl)1097-0061(199910)15:14<1555::AIDYEA479>3.0.CO;2-Z

van den Berg, M. A. (2010). Functional characterisation of penicillin production strains. Fungal Biology Reviews, 24(1-2), 73-78. https://doi.org/10.1016/j.fbr.2010.03.006

van Ooyen, A. J. J., Dekker, P., Huang, M., Olsthoorn, M. M. A., Jacobs, D. I., Colussi, P. A., \& Taron, C. H. (2006). Heterologous protein production in the yeast Kluyveromyces lactis. FEMS Yeast Research, 6(3), 381-392. https://doi.org/10.1111/j.1567-1364.2006.00049.x

van Roermund, C. W., Drissen, R., van Den Berg, M., ljlst, L., Hettema, E. H., Tabak, H. F., Waterham, H. R., \& Wanders, R. J. (2001). Identification of a peroxisomal ATP carrier required for medium-chain fatty acid beta-oxidation and normal peroxisome proliferation in Saccharomyces cerevisiae. Molecular and Cellular Biology, 21(13), 4321-4329. https://doi.org/10.1128/MCB.21.13.4321-4329.2001

van Roermund, C. W., Tabak, H. F., van Den Berg, M., Wanders, R. J., \& Hettema, E. H. (2000). Pex11p plays a primary role in medium-chain fatty acid oxidation, a process that affects peroxisome number and size in Saccharomyces cerevisiae. The Journal of Cell Biology, 150(3), 489-498. http://www.ncbi.nlm.nih.gov/pubmed/10931862

van Rossum, H. M., Kozak, B. U., Pronk, J. T., \& van Maris, A. J. A. (2016). Engineering cytosolic acetyl-coenzyme A supply in Saccharomyces cerevisiae: Pathway stoichiometry, free-energy conservation and redox-cofactor balancing. Metabolic Engineering, 36, 99-115. https://doi.org/10.1016/j.ymben.2016.03.006

Viegas, C. A., \& Sá-Correia, I. (1997). Effects of low temperatures $\left(9-33^{\circ} \mathrm{C}\right)$ and $\mathrm{pH}(3.3-5.7)$ in the loss of Saccharomyces cerevisiae viability by combining lethal concentrations of ethanol with octanoic and decanoic acids. International Journal of Food Microbiology, 34(3), 267-277. https://doi.org/10.1016/S0168-1605(96)01200-7

Viegas, C. A., Almeida, P. F., Cavaco, M., \& Sá-Correia, I. (1998). The H(+)-ATPase in the plasma membrane of Saccharomyces cerevisiae is activated during growth latency in octanoic acidsupplemented medium accompanying the decrease in intracellular $\mathrm{pH}$ and cell viability. 
Applied and Environmental Microbiology, 64(2), 779-783. http://www.ncbi.nlm.nih.gov/pubmed/9464423

Viegas, C. A., Rosa, M. F., Sá-Correia, I., \& Novais, J. M. (1989). Inhibition of Yeast Growth by Octanoic and Decanoic Acids Produced during Ethanolic Fermentation. Applied and Environmental Microbiology, 21 55(1), https://www.ncbi.nlm.nih.gov/pmc/articles/PMC184048/pdf/aem00094-0041.pdf

Viljoen, B. C., Kock, J. L. F., \& Lategan, P. M. (1986). Long-chain fatty acid composition of selected genera of yeasts belonging to the Endomycetales. Antonie van Leeuwenhoek, 52(1), 45-51. https://doi.org/10.1007/BF00402686

Wagner, J. M., Liu, L., Yuan, S. F., Venkataraman, M. V., Abate, A. R., \& Alper, H. S. (2018). A comparative analysis of single cell and droplet-based FACS for improving production phenotypes: Riboflavin overproduction in Yarrowia lipolytica. Metabolic Engineering, 47(February), 346-356. https://doi.org/10.1016/j.ymben.2018.04.015

Wang, B. L., Ghaderi, A., Zhou, H., Agresti, J., Weitz, D. A., Fink, G. R., \& Stephanopoulos, G. (2014). Microfluidic high-throughput culturing of single cells for selection based on extracellular metabolite production or consumption. Nature Biotechnology, 32(5), 473-478. https://doi.org/10.1038/nbt.2857

Wang, Z., Gerstein, M., \& Snyder, M. (2009). RNA-Seq: A revolutionary tool for transcriptomics. Nature Reviews Genetics, 10(1), 57-63. https://doi.org/10.1038/nrg2484

Weinberg, D. E., Shah, P., Eichhorn, S. W., Hussmann, J. A., Plotkin, J. B., \& Bartel, D. P. (2016). Improved Ribosome-Footprint and mRNA Measurements Provide Insights into Dynamics and Regulation of Yeast Translation. Cell Reports, 14(7), 1787-1799. https://doi.org/10.1016/j.celrep.2016.01.043

Wenz, P., Schwank, S., Hoja, U., \& Schüller, H. J. (2001). A downstream regulatory element located within the coding sequence mediates autoregulated expression of the yeast fatty acid synthase gene FAS2 by the FAS1 gene product. Nucleic Acids Research, 29(22), 4625-4632. https://doi.org/10.1093/nar/29.22.4625

Wernig, F., Baumann, L., Boles, E., \& Oreb, M. (2021). Production of octanoic acid in Saccharomyces cerevisiae: Investigation of new precursor supply engineering strategies and intrinsic limitations. Submitted.

Wernig, F., Boles, E., \& Oreb, M. (2019). De novo biosynthesis of 8-hydroxyoctanoic acid via a medium-chain length specific fatty acid synthase and cytochrome P450 in Saccharomyces cerevisiae. Metabolic Engineering Communications, e00111. https://doi.org/10.1016/j.mec.2019.e00111

Wernig, F., Born, S., Boles, E., Grininger, M., \& Oreb, M. (2020). Fusing $\alpha$ and $\beta$ subunits of the fungal fatty acid synthase leads to improved production of fatty acids. Scientific Reports, 10(1), 9780. https://doi.org/10.1038/s41598-020-66629-y

Wilson, T. A., Kritchevsky, D., Kotyla, T., \& Nicolosi, R. J. (2006). Structured triglycerides containing caprylic (8:0) and oleic (18:1) fatty acids reduce blood cholesterol concentrations and aortic cholesterol accumulation in hamsters. Biochimica et Biophysica Acta - Molecular and Cell Biology of Lipids, 1761(3), 345-349. https://doi.org/10.1016/j.bbalip.2006.02.019

Woods, A., Munday, M. R., Scott, J., Yang, X., Carlson, M., \& Carling, D. (1994). Yeast SNF1 is functionally related to mammalian AMP-activated protein kinase and regulates acetyl-CoA carboxylase in vivo. Journal of Biological Chemistry, 269(30), 19509-19515. https://doi.org/10.1016/S0021-9258(17)32198-1

Woolston, B. M., Edgar, S., \& Stephanopoulos, G. (2013). Metabolic Engineering: Past and Future. Annu. Rev. Chem. Biomol. Eng, 4, 259-288. https://doi.org/10.1146/annurev-chembioeng061312-103312

Xu, Q., Xue, C., Zhang, Y., Liu, Y., Wang, J., Yu, X., Zhang, X., Zhang, R., Yang, X., \& Guo, C. (2013). Medium-Chain Fatty Acids Enhanced the Excretion of Fecal Cholesterol and Cholic Acid in C57BL/6J Mice Fed a Cholesterol-Rich Diet. Biosci. Biotechnol. Biochem, 77(7), 13901396. https://doi.org/10.1271/bbb.120999 
Xue, P., Si, T., Mishra, S., Zhang, L., Choe, K., Sweedler, J. V., \& Zhao, H. (2020). A mass spectrometry-based high-throughput screening method for engineering fatty acid synthases with improved production of medium-chain fatty acids. Biotechnology and Bioengineering, 117(7), 2131-2138. https://doi.org/10.1002/bit.27343

Yan, Q., \& Pfleger, B. F. (2020). Revisiting metabolic engineering strategies for microbial synthesis of oleochemicals. Metabolic Engineering, 58, 35-46. https://doi.org/10.1016/j.ymben.2019.04.009

Yuan, Y., Arondel, V., \& Domergue, F. (2019). Characterization and heterologous expression of three DGATs from oil palm (Elaeis guineensis) mesocarp in Saccharomyces cerevisiae. Biochimie. https://doi.org/10.1016/J.BIOCHI.2019.09.010

Zhang, F., Carothers, J. M., \& Keasling, J. D. (2012). Design of a dynamic sensor-regulator system for production of chemicals and fuels derived from fatty acids. Nature Biotechnology, 30, 354359. https://doi.org/10.1038/nbt.2149

Zheng, Y., Chen, L., Zhu, Z., Li, D., \& Zhou, P. (2020). Multigene engineering of medium-chain fatty acid biosynthesis in transgenic Arabidopsis thaliana by a Cre/LoxP multigene expression system. 3 Biotech, 10(8). https://doi.org/10.1007/s13205-020-02340-z

Zhou, Y. J., Buijs, N. A., Zhu, Z., Qin, J., Siewers, V., \& Nielsen, J. (2016). Production of fatty acidderived oleochemicals and biofuels by synthetic yeast cell factories. Nature Communications, 7, 11709. https://doi.org/10.1038/ncomms 11709

Zhu, Z., Hu, Y., Teixeira, P. G., Pereira, R., Chen, Y., Siewers, V., \& Nielsen, J. (2020). Multidimensional engineering of Saccharomyces cerevisiae for efficient synthesis of mediumchain fatty acids. Nature Catalysis, 3(1), 64-74. https://doi.org/10.1038/s41929-019-0409-1

Zhu, Z., Zhou, Y. J., Krivoruchko, A., Grininger, M., Zhao, Z. K., \& Nielsen, J. (2017). Expanding the product portfolio of fungal type I fatty acid synthases. Nature Chemical Biology, 13, 360362. https://doi.org/10.1038/nCHeMBIO.2301

Zou, Z., DiRusso, C. C., Ctrnacta, V., \& Black, P. N. (2002). Fatty acid transport in Saccharomyces cerevisiae. Directed mutagenesis of FAT1 distinguishes the biochemical activities associated with Fat1p. The Journal of Biological Chemistry, 277(34), 31062-31071. https://doi.org/10.1074/jbc.M205034200 


\section{Additional Results}

\subsection{Additional results and discussion - Strategies to increase Saccharomyces cerevisiae robustness}

Octanoic acid has previously been shown to exert toxic effects on yeast. The toxicity of octanoic acid is attributed to different mechanisms. Octanoic acid is a lipophilic weak acid that crosses the yeast plasma membrane by passive diffusion in the protonated state or active transport and can dissociate in the cytosol. This leads to a decrease of $\mathrm{pH}$ in the cytosol and an accumulation of toxic anions. Furthermore, octanoic acid disturbs the membrane's organization and integrity. This perturbs the transport system of the membrane and its function as barrier (Viegas et al., 1998; Cabral et al., 2001; Legras et al., 2010; Liu et al., 2013).

Several strategies have been tested to counteract toxicity and increase robustness and will be described in the following. The toxicity of octanoic acid on a variety of $S$. cerevisiae strains was evaluated and ALE with the aim of increasing strain robustness was performed. In addition, rational engineering of targets via overexpression and knockout was evaluated regarding effects on tolerance.

\subsubsection{Evaluation of octanoic acid toxicity on Saccharomyces cerevisiae strains}

To assess growth inhibition, five $S$. cerevisiae strains were supplied with a range of octanoic acid concentrations under different conditions. The common haploid laboratory strain CEN.PK2-1C and four diploid industrial strains, i.e. IndSc-1, IndSc-2, IndSc-3, and EthanolRed, were tested. Firstly, pre-cultures of all strains were inoculated in triplicate shake flask cultures in YPD. In addition, cultures of all strains were preadapted to $200 \mathrm{mg} / \mathrm{L}$ octanoic acid in two consecutive rounds of overnight cultures in YPD. For evaluating growth inhibition, all shake flask cultures were cultivated until exponential growth phase was reached, diluted in fresh media and incubated with $0-800 \mathrm{mg} / \mathrm{L}$ octanoic acid in a 96-well plate without shaking. After $20 \mathrm{~h}$, the $\mathrm{OD}_{600}$ was measured in a platereader (Figure 5). 

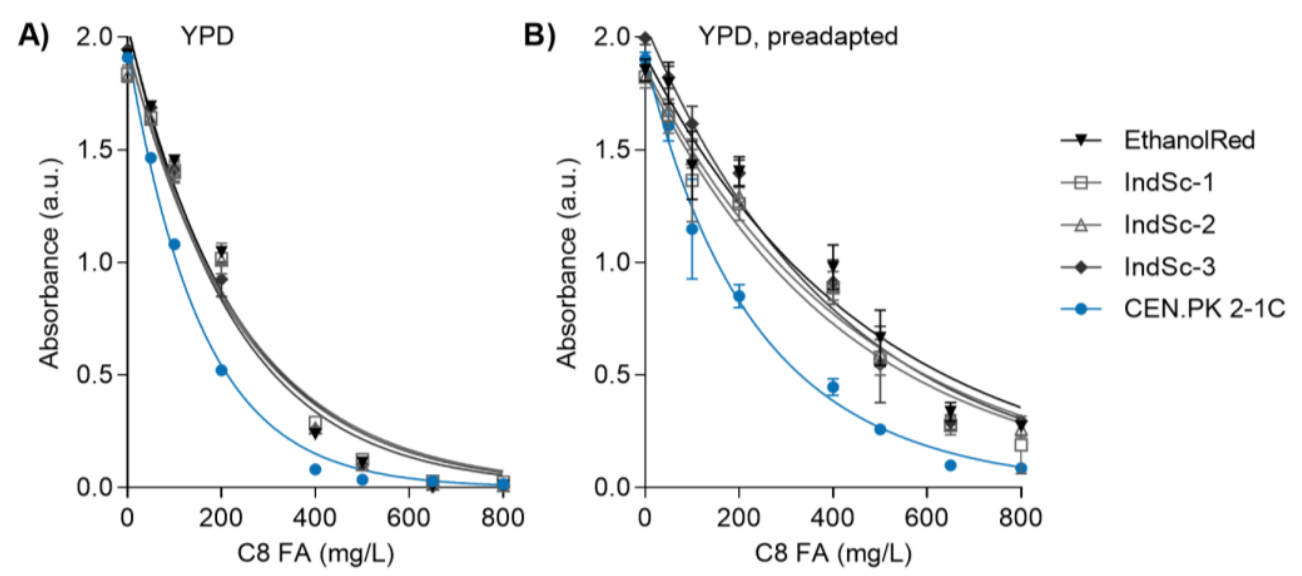

Figure 5. Assessing octanoic acid toxicity on industrial S. cerevisiae strains. Strains were inoculated to an OD600 of 0.05 and supplied with octanoic acid in (A) YPD or (B) YPD with two rounds of preadaptation to $200 \mathrm{mg} / \mathrm{L}$ octanoic acid before the test. Growth was measured by absorbance after $20 \mathrm{~h}$ (no agitation) in a platereader. $n=2$, error bars $= \pm$ standard deviation. Growth curves estimated with nonlinear regression, $\mathrm{R}^{2}>0.95$.

From this experiment several important conclusions could be drawn. Firstly, a preadaptation to octanoic acid led to better growth of all strains. Preadaptation to octanoic acid has previously been shown to be beneficial. It was suspected that protective changes in the membrane had occurred during adaptation and were responsible for decreased toxicity (Liu et al., 2013). Secondly, all industrial strains grew better than the laboratory strain CEN.PK2-1C under both conditions. Thirdly, octanoic acid inhibition is dose dependent. Increasing octanoic acid concentrations led to decreased growth. For non-adapted strains, 400-600 mg/L octanoic acid led to total growth arrest in YPD and for preadapted strains in YPD, $800 \mathrm{mg} / \mathrm{L}$ octanoic acid led to almost entire growth arrest.

To confirm that differences in growth not only occur in non-shaking conditions, CEN.PK2-1C and EthanolRed were cultivated in shake flask cultures in YPD medium supplemented with 0, 100, 200 and $400 \mathrm{mg} / \mathrm{L}$ octanoic acid, respectively, for $40 \mathrm{~h}$ with shaking on a cell growth quantifier (Aquila Biolabs). EthanolRed was chosen to represent the industrial strains as in the first experiment no difference between them had been observable. Again, better growth of EthanolRed than CEN.PK2-1C was observable under all octanoic acid concentrations and even without octanoic acid challenge (Figure 6). 


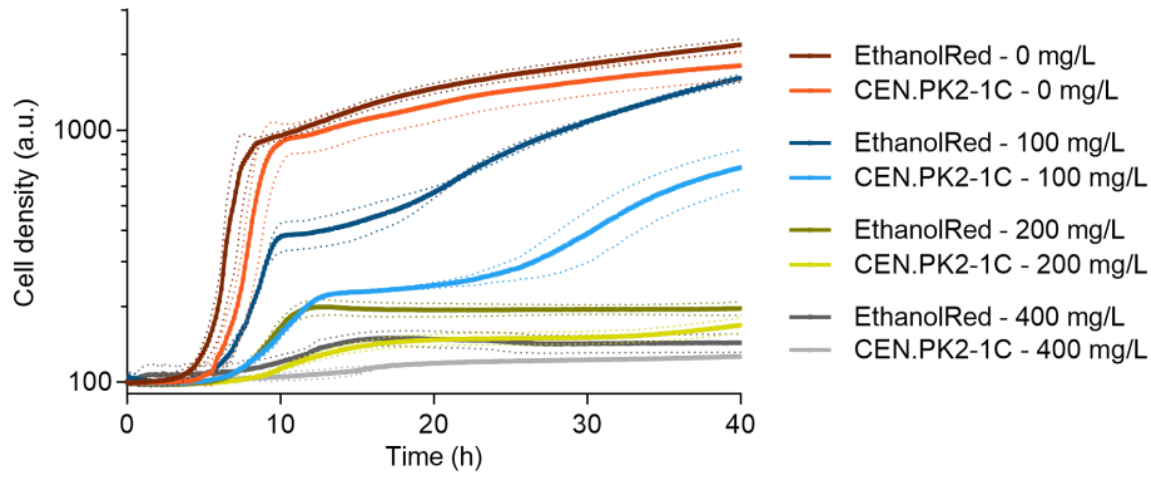

Figure 6. Assessing growth of CEN.PK2-1C and EthanolRed under octanoic acid challenge. Strains were inoculated to an $\mathrm{OD}_{600}$ of 0.1 in YPD medium in shake flasks and supplied with octanoic acid. Growth was measured continuously by a cell growth quantifier over 40 h. $n=3$, error bars $= \pm$ standard deviation.

In summary, S. cerevisiae industrial strains were more robust to octanoic acid than the haploid CEN.PK2-1C laboratory strain. However, creating and analyzing genetic modifications as well as general handling are much easier with haploid strains. For this reason, CEN.PK2-1C is commonly used in primary experiments to establish a production pathway and evaluate molecular modifications. Once a good laboratory strain has been established for producing high titers, the genetic modifications can be transferred to an industrial strain such as EthanolRed.

\subsubsection{Adaptive laboratory evolution and engineering of identified target genes}

ALE is a useful method to increase a strain's robustness against a toxic compound. This method is introduced in chapter 2.3.3. ALE is usually achieved by exposing the cells to increasing concentrations of the compound-of-interest in serial batch cultures. A slight variation of this procedure is to alternate the cultivation cycles in the presence and absence of the compound, which was shown to confer constitutive tolerance (González-Ramos et al., 2016). Both approaches were tested with the aim to generate a yeast strain that is more robust to octanoic acid. As a starting strain, a prototrophic $S$. cerevisiae strain was used in which $\beta$ oxidation was knocked out by deletion of POX1. Without preventing the degradation of fatty acids, it is likely that one would evolve and select for strains that grow better in the presence of octanoic acid due to enhanced degradation. 
The resulting strain LBY3 (CEN.PK113-7D $\Delta$ pox1) was cultivated in YPD medium spiked with $525 \mathrm{mg} / \mathrm{L}$ octanoic acid in triplicates for each of the two approaches (steadily increasing/ alternating concentrations) over three months (approximately 60 generations). Cultures were transferred to fresh media every 2-4 days and octanoic acid concentrations were increased three times over the course of the experiment (Figure 7A). An aliquot of each of the six final cultures as well as the original strain LBY3 were inoculated in fresh YPD medium and cultivated in the presence of $650 \mathrm{mg} / \mathrm{L}$ and $750 \mathrm{mg} / \mathrm{L}$ octanoic acid, respectively (Figure 7B).

The increased growth rate of all cultivated strains seemed to suggest that they had evolved for faster growth in the presence of high octanoic acid concentrations. For the next verification step, single colonies were isolated from the evolved cultures. This step is essential for whole genome sequencing, to be able to pinpoint genetic changes responsible for the improved phenotype. Several colonies were isolated from each culture and cultivated again in the presence of $650 \mathrm{mg} / \mathrm{L}$ octanoic acid. The growth curves are shown for exemplary colonies in Figure 7C. Only about half of all colonies showed higher growth rates than the parental strain and the difference between evolved colonies and the parental strain was much smaller as for the mixed cultures and mostly not significant.

Possibly, the mixed cultures contained cells with different genotypes that complemented each other and thereby enabled increased growth rates. However, it would be uneconomical to screen and sequence dozens to hundreds of single colonies to get a picture of the genotypic changes present in all six mixed cultures. Therefore, the focus was set on an alternative strategy. 


\section{Constant supply}

A)

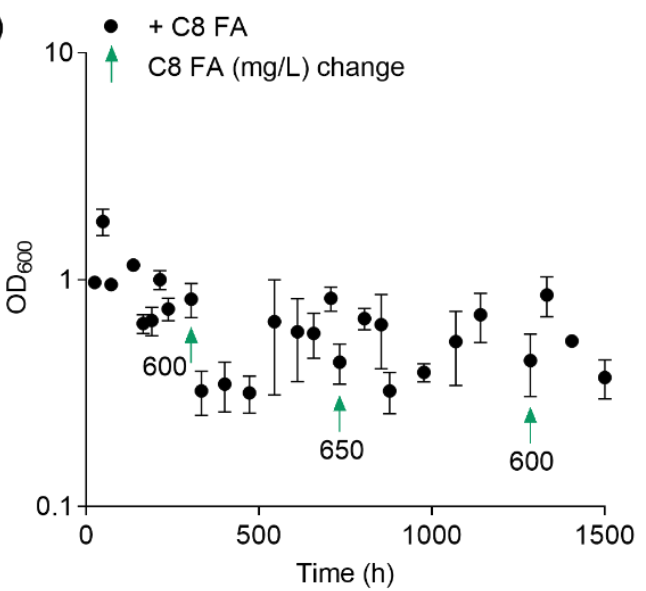

B)

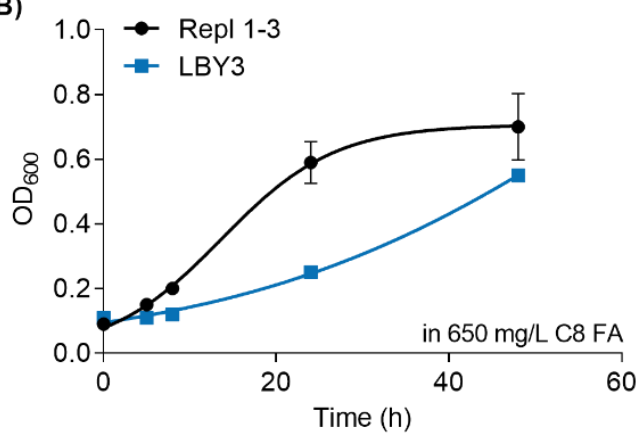

C)

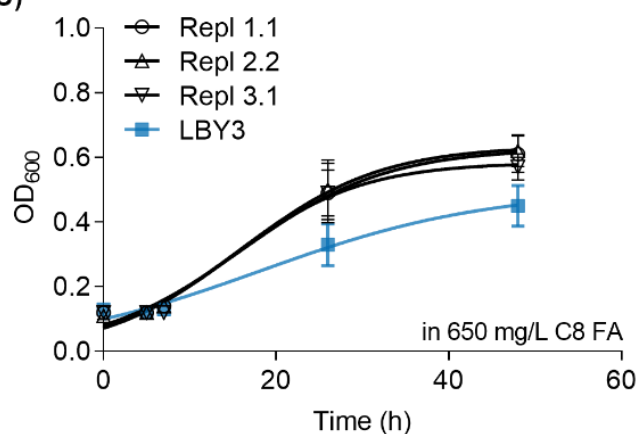

\section{Alternating supply/no supply}
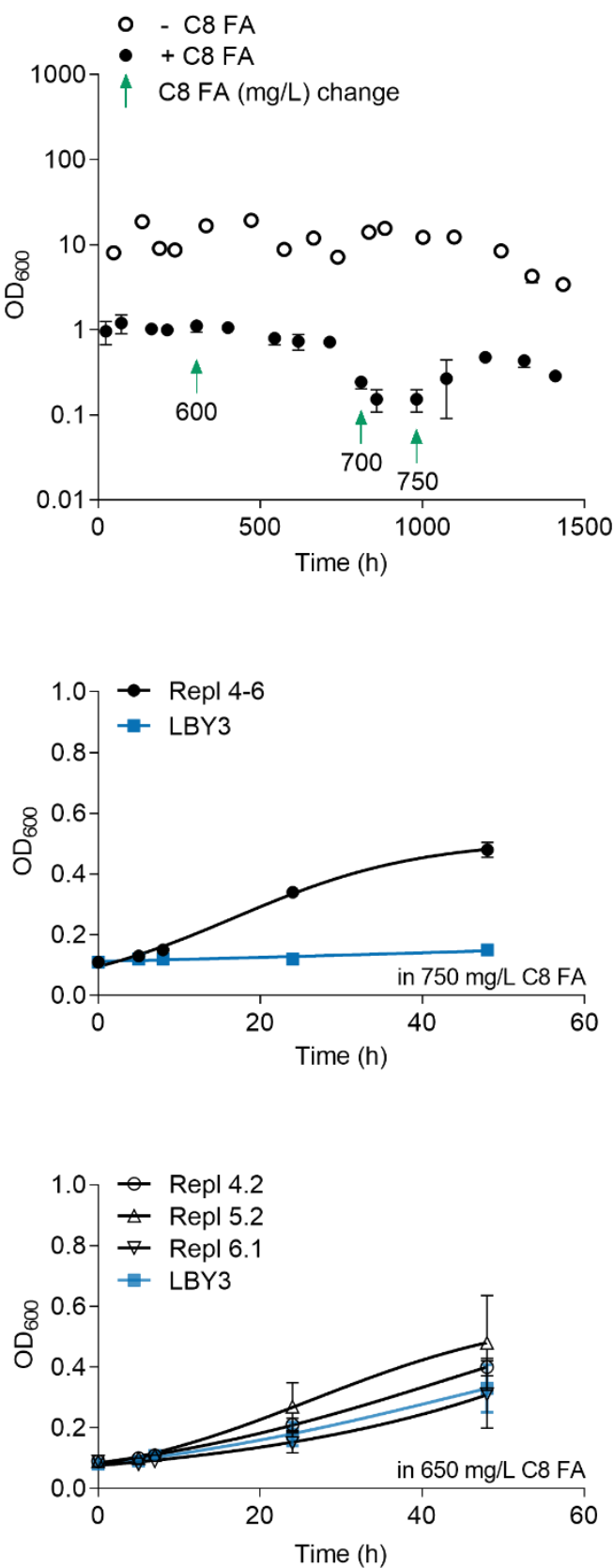

Figure 7. Adaptive laboratory evolution procedure and assessment of evolved strains. ALE was performed with strain LBY3 (CEN.PK113-7D $\Delta$ pox1) under two conditions with three replicates (Repl) each: Constant octanoic acid supply (left panel) and an alternating approach (right panel). (A) Both experiments were performed in YPD medium with a starting concentration of $525 \mathrm{mg} / \mathrm{L}$ octanoic acid which was adjusted over the course of ALE as indicated with arrows. (B) Evolved mixed cultures from the six parallel flask cultivations (Replicates 1-3 and 4-6, respectively) were grown with indicated octanoic acid amounts to verify improved evolved phenotypes. (C) Exemplary growth of cultures originating from single colonies of the mixed evolved population from each flask. 
In parallel to this thesis another group conducted an ALE experiment with octanoic acid (Zhu et al., 2020) similar to the one presented here. As parental strain they used an S. cerevisiae CEN.PK113-11C $\Delta p o x 1$ strain which was grown with octanoic acid over approximately 100 generations in parallel shake flasks in Delft medium. At the final stages of the ALE experiment, the cultures were supplied with $500 \mathrm{mg} / \mathrm{L}$ octanoic acid. The growth rates and final biomasses were increased in several evolved strains compared to the parental strain. Whole genome sequencing revealed mutations in seven genes, two of which were described to be causal for the observed phenotype. Combined knockout of the affected genes $P D R 1$ and $O S H 2$ resulted in increased resistance close to that of the final evolved strain and even separate knockout of each gene individually increased resistance markedly. PDR1 encodes a TF that regulates expression of multidrug resistance genes and $\mathrm{OSH} 2$ encodes a member of an oxysterol-binding protein family that is involved in sterol metabolism. The same group also reported a new variant of the transporter Tpo1, namely Tpo1-M49, which contains three amino acid exchanges and was obtained via directed evolution for decanoic acid tolerance. Tpo1-M49 was shown to also increase tolerance of cells to octanoic acid and lead to increased medium-chain fatty acid titers (Zhu et al., 2020).

Intrigued by this report, the aim was set to test the effect of $\Delta p d r 1, \Delta o s h 2$ and Tpo1M49 on robustness as well as octanoic acid production in the octanoic acid producer strain LBY38 ( $F A S 1^{R K}, \triangle$ faa2). Knockout mutants were generated in the LBY38 strain background, namely LBY42 (=LBY38 $\Delta$ pdr1) and LBY45 (=LBY38 $\Delta$ osh2) and genomically introduced TPO1-M49 into the pyk2 locus of LBY38 (=LBY44). To test the robustness of the strains, they were supplied with $0-325 \mathrm{mg} / \mathrm{L}$ octanoic acid and growth was measured after $42 \mathrm{~h}$ (Figure 8A). In addition, octanoic acid production of the strains was tested in YPD and SCD ${ }^{H T L U}$ medium, respectively, (Figure 8B, C) because increased robustness to octanoic acid eventually would only be a benefit if it also leads to elevated titers. 
A)

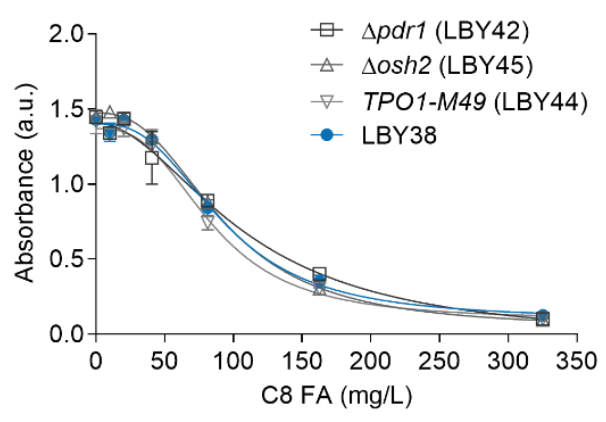

B)

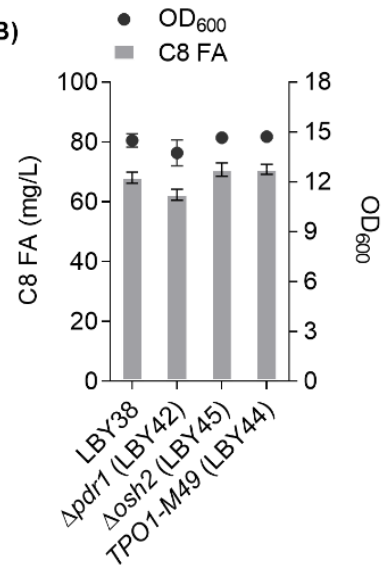

C)

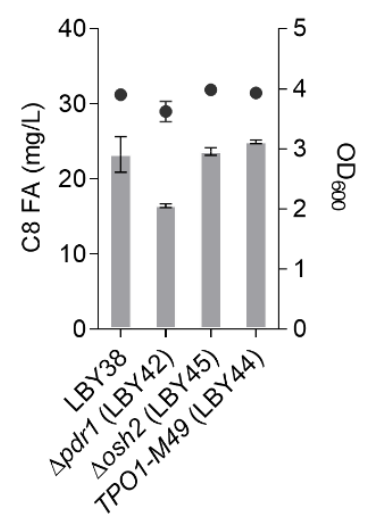

Figure 8. Influence of engineered targets on octanoic acid titers and toxicity. (A) Strains were inoculated to an $\mathrm{OD}_{600}$ of 0.05 and supplied with octanoic acid in buffered SCD ${ }^{H T L U}$ medium. Growth was measured by absorbance after $42 \mathrm{~h}$ (no agitation) in a platereader. $n=3$, error bars $=$ \pm standard deviation. (B, C) OD600 (dots) and octanoic acid titers (bars) of engineered strains compared to parental strain LBY38. Fatty acids were extracted, methylated and quantified via GC $48 \mathrm{~h}$ after inoculation in buffered YPD (B) and buffered SCD ${ }^{H T L U}$ medium (C), respectively. $n=2$, error bars $= \pm$ standard deviation

Surprisingly, none of the strains showed improved growth or production over the parental strain LBY38. The pdr1 knockout even led to decreased growth and octanoic acid titers in both SCD ${ }^{H T L U}$ and YPD medium. The reason for the differing phenotypes reported by Zhu and colleagues could possibly be attributed to differing experimental conditions such as media (Delft vs. SC/YP medium) or strain background. The knockouts of $P D R 1$ and $O S H 2$, respectively, were all generated in CEN.PK background strains, however, our knockouts were introduced into a producer strain whereas Zhu et al. introduced them in non-producer strains for toxicity tests. Possibly, this led to varying responses to supplied octanoic acid.

Here, one copy of Tpo1-M49 was integrated genomically into a strain that still contains the wild type TPO1 version and production and tolerance were tested in buffered SCD ${ }^{H T L U}$ medium. Zhu et al. (2020), on the other hand, showed improved tolerance for a $\triangle$ tpo1-TPO1-M49 strain in CSM medium. The increase in octanoic acid production was shown to depend on the copy number of TPO1-M49. Therefore, testing the effect of several copies of TPO1-M49 in a LBY38 $\Delta$ tpo 1 strain could be worthwhile. Furthermore, the TPO1-M49 variant was detected in a screening for improved decanoic acid transport, hence, a similar screening could be conducted to detect improved variants specifically for octanoic acid transport. 
In summary, the outcome of an ALE experiment is dependent on the individual experimental setup, evolved phenotypes of mixed cultures are not always detectable after single colony isolation, and if detectable, not necessarily confer the same phenotype in another strain background and experimental setup.

\subsubsection{Overexpression of transporters, transcriptional regulators and mutated acetyl-CoA carboxylase}

Previous to this thesis, several enzymes had been described to be involved in the response to, or transport of octanoic acid. Therefore, the encoding genes were presumed to be promising targets for rational engineering. The transporters Pdr12 and Tpo1 have been shown to be involved in octanoic acid expulsion from yeast cells (Legras et al., 2010; Borrull et al., 2015). The expression of PDR12 was found upregulated 3.5 -fold in wild type $S$. cerevisiae cells under octanoic acid challenge and a $\Delta p d r 12$ strain was more sensitive to octanoic acid than the wild type strain. Knockout of another transporter, $\Delta t p 01$, also resulted in increased sensitivity to octanoic acid (Legras et al., 2010). It was hypothesized that increased amounts of transporters could lead to enhanced octanoic acid expulsion and therefore better resistance and consequently growth.

Legras et al. (2010) also found that knockout of the transcriptional regulatorencoding genes WAR1 and PDR3 resulted in reduced growth when octanoic acid was supplied. War1 and Pdr3 are TFs that regulate the expression of transporters that could be involved in octanoic acid export. War1 is a positive regulator of PDR12 expression in response to weak acids including octanoic acid (Holyoak et al., 1999; Hatzixanthis et al., 2003; Kren et al., 2003). Pdr3 was shown to regulate the expression of $A B C$ transporters that are involved in multidrug resistance (Schüller et al., 2007; Thakur et al., 2008). The fifth chosen target for overexpression was a mutated version of the acetyl-CoA carboxylase encoding ACC1 gene. It was reported that a mutated version, namely Acc1 ${ }^{\text {S1157A }}$, conferred increased resistance to octanoic acid due to an increase in oleic acid in the membrane (Besada-Lombana et al., 2017), similar to the effect of externally supplied oleic acid (Liu et al., 2013). 
The overexpression of PDR12 did not result in increased growth or octanoic acid titers in our hands (Baumann et al., 2021). To test the effect of overexpression of the four other targets, they were expressed under the control of medium strong promotors on multicopy plasmids in the non-producer strain CEN.PK113-7D and resistance to supplied octanoic acid was evaluated (Figure 9).

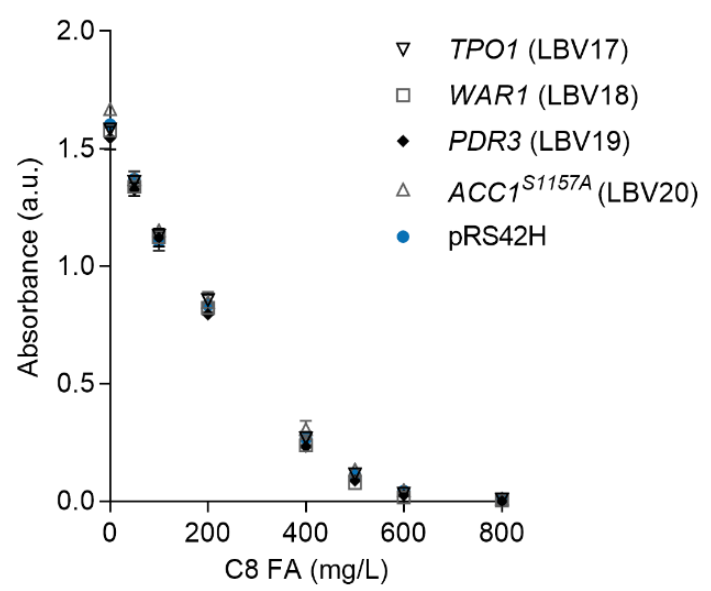

Figure 9: Influence of overexpressed genes on growth of CEN.PK113-7D with octanoic acid supply. CEN.PK113-7D was transformed with plasmids containing the indicated genes or the control plasmid (pRS42H), respectively. All strains were inoculated to an $\mathrm{OD}_{600}$ of 0.05 and supplied with octanoic acid in buffered SCD hygro medium. Growth was measured by absorbance after $20 \mathrm{~h}$ (no agitation) in a platereader. $n=3$, error bars $= \pm$ standard deviation

The experiment showed that neither of the overexpressions led to an increase in resistance to octanoic acid in the non-producer background CEN.PK113-7D. For the overexpression of the transporter-encoding genes PDR12 and TPO1 and the TF-encoding WAR1 (regulating PDR12 expression) this could be a hint that export of octanoic acid is not a bottleneck for detoxification - at least not at near neutral $\mathrm{pH}(\sim 6.5)$. It is possible that the active export of octanoic acid plays a minor role in comparison to passive diffusion. Furthermore, the toxicity test showed no effect of PDR3 overexpression on resistance to octanoic acid. This suggests that the transporters under control of Pdr3 do not mediate octanoic acid transport. However, considering that $\Delta$ war1 and $\Delta p d r 3$ strains exerted reduced growth under octanoic acid supplementation (Legras et al., 2010), it is more likely that native expression levels of involved transporters are sufficient. Another possibility is that active export becomes important only at low $\mathrm{pH}$, when the protonated form can easily enter the cell and rapid active export becomes a bottleneck for detoxification.

In contrast to previous reports, a positive effect from ACC151157A expression on octanoic acid resistance was not observable. The "S1157A" amino acid exchange was described to abolish Snf1-mediated repression of Acc1 (Choi \& Da Silva, 2014; Shi et al., 2014), leading to increased resistance to octanoic acid (Besada- 
Lombana et al., 2017). The contradictory results could be due to the expression of the native $A C C 1$ from the strain background, which might mask positive effects expected from expression of the mutated version.

\subsubsection{Conclusion}

The results presented above show that toxicity is a complex trait to tackle. The aim of the presented approaches was to assess the inherent robustness of different $S$. cerevisiae strains to determine the most suitable production host. Furthermore, ALE and engineering of rational targets were to confer an $S$. cerevisiae strain with increased resistance to octanoic acid.

A robust yeast strain is essential for industrial octanoic acid production in which high titers would need to be produced. The here presented approaches were not effective in increasing robustness. However, it was shown that industrial yeast strains, like EthanolRed, are suitable candidates as future production hosts due to increased tolerance to octanoic acid, especially after preadaptation. Nevertheless, even for industrial strains, octanoic acid toxicity is a vital issue that needs to be minimized. To achieve this goal, future work should focus on evaluating other approaches, for instance, engineering membrane composition, in situ extraction of octanoic acid, or transformation of octanoic acid into non-toxic derivatives as discussed in detail in chapter 3.3. 


\subsection{Material and methods}

\subsubsection{Strains and plasmid construction}

Yeast strains and plasmid used in the additional results are listed in Table 1 and Table 2, respectively. Plasmids LBV18 and LBV19 and were constructed as follows: Promoters $p T P I 1$ and $p P Y K 1$ as well as genomic regions WAR1-tWAR1 and PDR3-tPDR3 were amplified from CEN.PK113-11C genomic DNA with primers containing respective overhangs (Table 3 ) for homologous recombination in yeast. Plasmid pRS42H was digested with EcoRV and transformed with the respective insertion fragments into CEN.PK2-1C for homologous recombination. Yeast transformations were performed according to Gietz \& Schiestl (2007). Cells were streaked out on YPD hygro $(200 \mu \mathrm{g} / \mathrm{mL})$ medium to select for hygromycinresistant colonies. All colonies on the selective plates were collectively transformed into electrocompetent E. coli DH10 $\beta$ (Gibco BRL, Gaithersburg, MD) and transformants were selected on lysogeny broth (LB) agar plates supplemented with $100 \mu \mathrm{g} / \mathrm{mL}$ ampicillin (Sambrook et al., 1989). Selected plasmids were extracted according to standard procedures and verified via Sanger sequencing.

Knockout of $P D R 1$ and $O S H 2$ and replacement of $P Y K 2$ open reading frame by TPO1-M49, respectively, were performed via CRISPR/Cas9. CRISPR/Cas9 plasmids were amplified each in two PCR fragments, assembled in vitro in an isothermal reaction using T5 exonuclease, polymerase and ligase (Gibson et al., 2009) and transformed into E. coli DH10ß. Plasmids were verified via Sanger sequencing. The insertion fragment TPO1-M49 was amplified from plasmid p416GPD-TPO1-M49 with overhangs for up- and downstream regions, respectively, of $P Y K 2$. CRISPR/Cas9 plasmids were transformed into LBY38 with the respective donor/insertion fragments. Clones were analyzed via genomic DNA extraction and PCR verification according to standard procedures. 
Table 1. Yeast strains used in the additional results.

\begin{tabular}{|c|c|c|}
\hline Strain & Characteristics & Reference \\
\hline CEN.PK2-1C & $\begin{array}{l}\text { MATa; MAL2-8c; SUC2; ura3-52; } \\
\text { his3A1; leu2-3_112; trp1-289 }\end{array}$ & $\begin{array}{l}\text { Euroscarf, Frankfurt am } \\
\text { Main, Germany }\end{array}$ \\
\hline CEN.PK113-7D & MATa; MAL2-8c; SUC2 & $\begin{array}{l}\text { Euroscarf, Frankfurt am } \\
\text { Main, Germany }\end{array}$ \\
\hline CEN.PK113-7D $\Delta$ pox 1 & MATa; MAL2-8c; SUC2; $\triangle p o x 1$ & (Wernig et al., 2019) \\
\hline CEN.PK113-11C & $\begin{array}{l}\text { MATa; MAL2-8c; SUC2; ura3-52; } \\
\text { his3D1 }\end{array}$ & $\begin{array}{l}\text { Euroscarf, Frankfurt am } \\
\text { Main, Germany }\end{array}$ \\
\hline Ind-Sc1 & 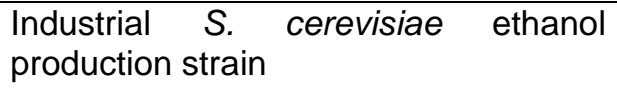 & This group \\
\hline Ind-Sc2 & $\begin{array}{l}\text { Industrial } S . \\
\text { production strain }\end{array}$ & This group \\
\hline Ind-Sc3 & 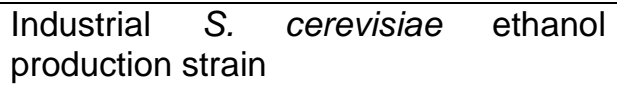 & This group \\
\hline EthanolRed & 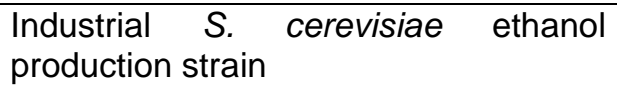 & Lesaffre/Fermentis \\
\hline LBY38 & $\begin{array}{l}\text { CEN.PK2-1C } \triangle p F A S 1-1-300:: p H X T 7- \\
1-392 \Delta p F A S 2-1-200:: p H X T 7-1-392, \\
\text { FAS1 } 11834 k, \triangle \text { faa2 }\end{array}$ & (Wernig et al., 2021) \\
\hline LBY42 & LBY38 $\Delta p d r 1$ & This thesis \\
\hline LBY44 & $\begin{array}{l}\text { LBY38 } \quad \Delta p y k 2:: p T D H 3-T P O 1-M 49 \\
(\text { (F322L, T54S, I432N)-tCYC1 }\end{array}$ & This thesis \\
\hline LBY45 & LBY38 $\Delta$ osh2 & This thesis \\
\hline
\end{tabular}

Table 2. Plasmids used in the additional results.

\begin{tabular}{|l|l|l|}
\hline Plasmid & Characteristics & Reference \\
\hline pRS42H & $\begin{array}{l}2 \mu, H P H^{R}, A m p R, \text { multiple cloning site including } \\
\text { EcoRV }\end{array}$ & (Taxis \& Knop, 2006) \\
\hline pRCC-K & $\begin{array}{l}2 \mu, \quad \text { kanMX, AmpR, pROX3-Cas9opt-tCYC1, } \\
p S N R 52-g R N A\end{array}$ & (Generoso et al., 2016) \\
\hline $\begin{array}{l}\text { p416GPD- } \\
\text { TPO1-M49 }\end{array}$ & $\begin{array}{l}\text { CEN6/ARS4, AmpR, URA3, pTDH3-TPO1(F322L, } \\
\text { T54S, I432N)-tCYC1 }\end{array}$ & (Zhu et al., 2020) \\
\hline LBV17 & $\begin{array}{l}\text { pRS42H with pPGK1-TPO1-tTPO1 integrated in } \\
\text { EcoRV site }\end{array}$ & (Baumann et al., 2018) \\
\hline LBV18 & $\begin{array}{l}\text { pRS42H with pFBA1-WAR1-tWAR1 integrated in } \\
\text { EcoRV site }\end{array}$ & This thesis \\
\hline LBV19 & $\begin{array}{l}\text { pRS42H with pTPI1-PDR3-tPDR3 integrated in } \\
\text { EcoRV site }\end{array}$ & This thesis \\
\hline LBV20 & $\begin{array}{l}\text { pRS42H with pPYK1-ACC1S1157A-tACC1 } \\
\text { integrated in EcoRV site }\end{array}$ & (Baumann et al., 2018) \\
\hline LBV42 & pRCC-K with gRNA for PDR1 locus & This thesis \\
\hline LBV43 & pRCC-K with gRNA for OSH2 locus & This thesis \\
\hline LBV103 & pRCC-K with gRNA for PYK2 locus & (Baumann et al., 2021) \\
\hline
\end{tabular}


Table 3. Oligonucleotides used in the additional results.

\begin{tabular}{|c|c|c|}
\hline Primer & Sequence $5^{\prime} \rightarrow 3^{\prime}$ & Application \\
\hline LBP92 & $\begin{array}{l}\text { GGTCGACGGTATCGATAAGCTTGATGCGGCCGCTGGGTCA } \\
\text { TTACGTAAATAATGATAGG }\end{array}$ & \multirow{6}{*}{$\begin{array}{l}\text { Amplification } \\
\text { and sequencing } \\
\text { of pFBA1 and } \\
\text { WAR1-tWAR1 } \\
\text { with overhangs } \\
\text { to } \mathrm{pRS} 42 \mathrm{H}\end{array}$} \\
\hline LBP93 & $\begin{array}{l}\text { CCAGTTATTGCAATCTGCGTGTCCATTTTGAATATGTATTAC } \\
\text { TTGGTTATGG }\end{array}$ & \\
\hline LBP94 & $\begin{array}{l}\text { CCATAACCAAGTAATACATATTCAAAATGGACACGCAGATT } \\
\text { GCAATAACTGG }\end{array}$ & \\
\hline LBP95 & $\begin{array}{l}\text { GCTGGAGCTCCACCGCGGTGGCGGCCGCCAACAACTTATT } \\
\text { ATCACCGTACGATTTCGC }\end{array}$ & \\
\hline LBP96 & GTTGAAGGGATACAACTCTG & \\
\hline LBP97 & GCTTCATAATAGTTCCTCTGG & \\
\hline LBP86 & $\begin{array}{l}\text { GAGGTCGACGGTATCGATAAGCTTGATGAGCTCGAATAGC } \\
\text { TCATCCAATCGATCCAAG }\end{array}$ & \multirow{6}{*}{$\begin{array}{l}\text { Amplification } \\
\text { and sequencing } \\
\text { of pTPI1 and } \\
P D R 3-t P D R 3 \\
\text { with overhangs } \\
\text { to } \mathrm{pRS} 42 \mathrm{H}\end{array}$} \\
\hline LBP87 & $\begin{array}{l}\text { CTACAAAAAACACATACATAAACTAAAAATGAAAGTGAAGA } \\
\text { AATCAACTAGATCAAAAG }\end{array}$ & \\
\hline LBP88 & $\begin{array}{l}\text { CTTTTGATCTAGTTGATTTCTTCACTTTCATTTTTAGTTTATG } \\
\text { TATGTGTTTTTTGTAG }\end{array}$ & \\
\hline LBP89 & $\begin{array}{l}\text { CCCTCACTAAAGGGAACAAAAGCTGGAGCTCGATTTAAACT } \\
\text { GTGAGGACCTTAATAC }\end{array}$ & \\
\hline LBP90 & CCTGATACTTCCAATAATCC & \\
\hline LBP91 & GTGTCATTGCTAGAAATCC & \\
\hline WGP234 & $\begin{array}{l}\text { CTTGGTGGTGTTCGTCGTATCTCTTAATCATAGAAGCAGAC } \\
\text { AATGGAG }\end{array}$ & \multirow{2}{*}{$\begin{array}{l}\text { Amplification of } \\
\text { CRISPR/Cas9 } \\
\text { plasmid } \\
\text { backbone }\end{array}$} \\
\hline WGP235 & $\begin{array}{l}\text { TGTTGTCTGACATTTTGAGAGTTAACACCGAAATTACCAAG } \\
\text { GCTC }\end{array}$ & \\
\hline MGP126 & GGGAAACGCCTGGTATC & $\begin{array}{l}\text { Sequencing of } \\
\text { gRNAs }\end{array}$ \\
\hline LBP259 & $\begin{array}{l}\text { CTGGATAAACGTCGCTCCACGTTTTAGAGCTAGAAATAGCA } \\
\text { AGTTAAAATAAGG }\end{array}$ & \multirow{2}{*}{$\begin{array}{l}\text { Amplification of } \\
\text { pRCC-K with } \\
\text { gRNA for PDR1 }\end{array}$} \\
\hline LBP260 & $\begin{array}{l}\text { GTGGAGCGACGTTTATCCAGGATCATTTATCTTTCACTGCG } \\
\text { GAG }\end{array}$ & \\
\hline LBP261 & $\begin{array}{l}\text { CAGCCAAGAATATACAGAAAAGAATCCAAGAAACTGGAAGA } \\
\text { CGTATACGTTTGTATAGATAAAAGTTCTCAAAAACTTCC }\end{array}$ & \multirow{2}{*}{$\begin{array}{l}\text { Donor DNA for } \\
\text { PDR1 knockout }\end{array}$} \\
\hline LBP262 & $\begin{array}{l}\text { GGAAGTTTTTGAGAACTTTTATCTATACAAACGTATACGTCT } \\
\text { TCCAGTTTCTTGGATTCTTTTCTGTATATTCTTGGCTG }\end{array}$ & \\
\hline LBP263 & TCAAAACAACCTTATTCGCC & \multirow{4}{*}{$\begin{array}{l}\text { Sequencing of } \\
\text { PDR1 locus }\end{array}$} \\
\hline LBP264 & ACAGACAGCATTAGACATTTC & \\
\hline LBP271 & AAATCACATCTAACCCCTCC & \\
\hline LBP272 & AGTAAGCATGAAGTGCCC & \\
\hline LBP265 & $\begin{array}{l}\text { ACTAGAACCGCTGCATCAGCGTTTTAGAGCTAGAAATAGCA } \\
\text { AGTTAAAATAAGG }\end{array}$ & \multirow{2}{*}{$\begin{array}{l}\text { Amplification of } \\
\text { pRCC-K with } \\
\text { gRNA for OSH2 }\end{array}$} \\
\hline LBP266 & $\begin{array}{l}\text { GCTGATGCAGCGGTTCTAGTGATCATTTATCTTTCACTGCG } \\
\text { GAG }\end{array}$ & \\
\hline LBP267 & $\begin{array}{l}\text { TAATCATAAGCTTAAGCTCGCCAACTGAAAACTTCACAATC } \\
\text { ACGCCTTTTTTATGCGAGCTTTTTTCCTGGTACTTGTAT }\end{array}$ & \multirow{2}{*}{$\begin{array}{l}\text { Donor DNA for } \\
\text { OSH2 knockout }\end{array}$} \\
\hline LBP268 & $\begin{array}{l}\text { ATACAAGTACCAGGAAAAAAGCTCGCATAAAAAAGGCGTG } \\
\text { ATTGTGAAGTTTTCAGTTGGCGAGCTTAAGCTTATGATTA }\end{array}$ & \\
\hline LBP276 & GTTATAACAAGGGCTCCTCC & \multirow{4}{*}{$\begin{array}{l}\text { Sequencing of } \\
\text { OSH2 locus }\end{array}$} \\
\hline LBP277 & GCCAAAAGAAACAAATGTGC & \\
\hline LBP273 & СCTCATCAACACATCAACC & \\
\hline LBP274 & TCATAACGCCCATCTTCC & \\
\hline
\end{tabular}




\begin{tabular}{|c|c|c|}
\hline LBP282 & $\begin{array}{l}\text { CAACTATATTTTACTTTCATCCTCTACGTCCATTGTAAGATT } \\
\text { ACAACAAAAGCACTATCGCGTCCCAAAACCTTCTCAAG }\end{array}$ & \multirow{4}{*}{$\begin{array}{l}\text { Amplification } \\
\text { and sequencing } \\
\text { of } \\
\triangle p y k 2:: p T D H 3- \\
T P O 1-M 49- \\
\text { tCYC1 }\end{array}$} \\
\hline LBP283 & $\begin{array}{l}\text { CTGACACAATGGACAATTAAATAAAATTAAGTAAAAAAAATA } \\
\text { AGGACTTTAATTTTTAACAGTTTATTCCTGGCATCC }\end{array}$ & \\
\hline LBP118 & CAGAGCGGTGAAACGCAAC & \\
\hline LBP119 & CGCAGTTTGCGAACATTACCTG & \\
\hline
\end{tabular}

\subsubsection{Cultivation of yeast strains}

For S. cerevisiae cultures, pre-cultures were inoculated from several colonies and combined in $20 \mathrm{~mL} \mathrm{SCD}$ (with amino acids histidine, tryptophan, lysin and uracil, if applicable) or YPD with or without $100 \mathrm{mM}$ potassium phosphate buffer $(\mathrm{pH} 6.5)$ as indicated for each experiment. After shaking at 180 r.p.m. at $30^{\circ} \mathrm{C}$ overnight, the main cultures were inoculated to an $\mathrm{OD}_{600}$ of 0.1 in $20-50 \mathrm{~mL}$ of the respective medium - containing octanoic acid if applicable - and cultured in $100 \mathrm{~mL}$ or $300 \mathrm{~mL}$ shake flasks under the same conditions. $\mathrm{OD}_{600}$ was measured in a spectrophotometer or a cell growth quantifier from Aquila biolabs as indicated for each Figure. For sampling, cultures were harvested after $48 \mathrm{~h}$ of fermentation by centrifugation and $10 \mathrm{~mL}$ of the supernatant was used for fatty acid extraction.

For the preadaptation to octanoic acid, precultures were inoculated from single colonies in YPD without buffer and grown overnight (180 r.p.m., $30^{\circ} \mathrm{C}$ ). Strains were inoculated to an OD of 0.1 to fresh YPD containing $200 \mathrm{mg} / \mathrm{L}$ octanoic acid and cultivated overnight. This procedure was performed twice before strains were used for the toxicity test (chapter 5.2.4).

\subsubsection{Fatty acid extraction, derivatization and GC-FID analysis}

Fatty acid extraction, derivatization and GC analysis were performed as described previously (Henritzi et al., 2018). Fatty acids were extracted from $10 \mathrm{~mL}$ culture supernatant after $48 \mathrm{~h}$ of cultivation, derivatized to FAMEs and measured on a Perkin Elmer Clarus 400 instrument (Perkin Elmer, Germany) equipped with an Elite FFAP capillary column $(30 \mathrm{~m} \times 0.25 \mathrm{~mm}$, film thickness: $0.25 \mu \mathrm{m}$; PerkinElmer, Germany) and a flame ionization detector (Perkin Elmer, Germany). 


\subsubsection{Toxicity tests}

Pre-cultures were inoculated in YPD or SCD medium (with supplements if applicable) as indicated for each experiment and grown overnight $\left(30^{\circ} \mathrm{C}, 180\right.$ r.p.m.). For main cultures, strains were inoculated to an $\mathrm{OD}_{600}$ of 0.2 and cultivated $\left(30^{\circ} \mathrm{C}, 180\right.$ r.p.m.) for 4-6 hours. Cultures were then diluted in fresh media to an $\mathrm{OD}_{600}$ of 0.05 and aliquots of $50 \mu$ transferred into wells of a 96-well plate (clear with flat bottom, greiner bio-one). An octanoic acid (Sigma-Aldrich, GC grade) dilution series was made in fresh media. $200 \mu \mathrm{l}$ aliquots of these octanoic acid dilutions were transferred into the wells containing the $50 \mu \mathrm{l}$ strain solutions to reach final volumes of $250 \mu \mathrm{l}$ per well. The starting absorbance was measured in a platereader (CLARIOstar ${ }^{\circledR}, \mathrm{BMG}$ Labtech, Ortenberg, Germany) and plates were incubated for the indicated time period at $30^{\circ} \mathrm{C}$ without shaking before absorbance was measured again. From final absorbance values, a blank value (media without strain) was subtracted.

\subsubsection{Adaptive laboratory evolution}

ALE was performed with strain LBY3 (CEN.PK113-7D $\Delta$ pox1) in two parallel approaches. For both approaches, three shake flasks each were used (180 r.p.m., $30^{\circ} \mathrm{C}$ ). At the beginning, LBY3 was inoculated to an $\mathrm{OD}_{600}$ of 0.01 in all six flasks in YPD medium supplied with $525 \mathrm{mg} / \mathrm{L}$ octanoic acid. Cultures were serially transferred to fresh medium every 2-4 days and growth was measured periodically with a spectrophotometer. In the first approach, octanoic acid was always present in the medium. For the second approach, every second transfer was performed into fresh medium without octanoic acid, thereby releasing toxicity pressure. The octanoic acid amounts were varied over the course of the experiments as indicated in Figure 7A. Evolved cultures were assessed as described in chapter 5.2.2.

\subsubsection{Software}

Data tables were stored in Microsoft Excel 2016. Graphs were made using the software Prism 9.0.0 (GraphPad, USA). Design of plasmids was accomplished with Geneious Prime 2020.2. 


\subsection{References for the additional results}

Baumann, L., Doughty, T., Siewers, V., Nielsen, J., Boles, E., \& Oreb, M. (2021). Transcriptomic response of Saccharomyces cerevisiae to octanoic acid production. FEMS Yeast Research (Accepted).

Baumann, L., Rajkumar, A. S., Morrissey, J. P., Boles, E., \& Oreb, M. (2018). A yeast-based biosensor for screening of short- and medium-chain fatty acid production. ACS Synthetic Biology, 7(11), 2640-2646. https://doi.org/10.1021/acssynbio.8b00309

Besada-Lombana, P. B., Fernandez-Moya, R., Fenster, J., \& Da Silva, N. A. (2017). Engineering Saccharomyces cerevisiae fatty acid composition for increased tolerance to octanoic acid. Biotechnology and Bioengineering, 114(7), 1531-1538. https://doi.org/10.1002/bit.26288

Borrull, A., López-Martínez, G., Poblet, M., Cordero-Otero, R., \& Rozès, N. (2015). New insights into the toxicity mechanism of octanoic and decanoic acids on Saccharomyces cerevisiae. Yeast, 32(5), 451-460. https://doi.org/10.1002/yea.3071

Cabral, M. G., Viegas, C. A., \& Sá-Correia, I. (2001). Mechanisms underlying the acquisition of resistance to octanoic-acid-induced-death following exposure of Saccharomyces cerevisiae to mild stress imposed by octanoic acid or ethanol. Archives of Microbiology, 175(4), 301307. http://www.ncbi.nlm.nih.gov/pubmed/11382226

Choi, J. W., \& Da Silva, N. A. (2014). Improving polyketide and fatty acid synthesis by engineering of the yeast acetyl-CoA carboxylase. Journal of Biotechnology, 187, 56-59. https://doi.org/10.1016/j.jbiotec.2014.07.430

Generoso, W. C., Gottardi, M., Oreb, M., \& Boles, E. (2016). Simplified CRISPR-Cas genome editing for Saccharomyces cerevisiae. Journal of Microbiological Methods, 127, 203-205. https://doi.org/10.1016/J.MIMET.2016.06.020

Gibson, D. G., Young, L., Chuang, R. Y., Venter, J. C., Hutchison, C. A., \& Smith, H. O. (2009). Enzymatic assembly of DNA molecules up to several hundred kilobases. Nature Methods, 6(5), 343-345. https://doi.org/10.1038/nmeth.1318

Gietz, R. D., \& Schiestl, R. H. (2007). High-efficiency yeast transformation using the LiAc/SS carrier DNA/PEG method. Nature Protocols, 2(1), 31-34. https://doi.org/10.1038/nprot.2007.13

González-Ramos, D., Gorter de Vries, A. R., Grijseels, S. S., van Berkum, M. C., Swinnen, S., van den Broek, M., Nevoigt, E., Daran, J.-M. G., Pronk, J. T., \& van Maris, A. J. A. (2016). A new laboratory evolution approach to select for constitutive acetic acid tolerance in Saccharomyces cerevisiae and identification of causal mutations. Biotechnology for Biofuels, 9, 173. https://doi.org/10.1186/s13068-016-0583-1

Hatzixanthis, K., Mollapour, M., Seymour, I., Bauer, B. E., Krapf, G., Schüller, C., Kuchler, K., \& Piper, P. W. (2003). Moderately lipophilic carboxylate compounds are the selective inducers of the Saccharomyces cerevisiae Pdr12p ATP-binding cassette transporter. Yeast, 20(7), 575-585. https://doi.org/10.1002/yea.981

Henritzi, S., Fischer, M., Grininger, M., Oreb, M., \& Boles, E. (2018). An engineered fatty acid synthase combined with a carboxylic acid reductase enables de novo production of 1octanol in Saccharomyces cerevisiae. Biotechnology for Biofuels, 11(150). https://doi.org/10.1186/s13068-018-1149-1

Holyoak, C. D., Bracey, D., Piper, P. W., Kuchler, K., \& Coote, P. J. (1999). The Saccharomyces cerevisiae weak-acid-inducible ABC transporter Pdr12 transports fluorescein and preservative anions from the cytosol by an energy-dependent mechanism. Journal of Bacteriology, 181(15), 4644-4652. http://www.ncbi.nlm.nih.gov/pubmed/10419965

Kren, A., Mamnun, Y. M., Bauer, B. E., Schüller, C., Wolfger, H., Hatzixanthis, K., Mollapour, M., Gregori, C., Piper, P., \& Kuchler, K. (2003). War1p, a novel transcription factor controlling weak acid stress response in yeast. Molecular and Cellular Biology, 23(5), 1775-1785. https://doi.org/10.1128/mcb.23.5.1775-1785.2003 
Legras, J. L., Erny, C., Le Jeune, C., Lollier, M., Adolphe, Y., Demuyter, C., Delobel, P., Blondin, B., \& Karst, F. (2010). Activation of two different resistance mechanisms in Saccharomyces cerevisiae upon exposure to octanoic and decanoic acids. Applied and Environmental Microbiology, 76(22), 7526-7535. https://doi.org/10.1128/AEM.01280-10

Liu, P., Chernyshov, A., Najdi, T., Fu, Y., Dickerson, J., Sandmeyer, S., \& Jarboe, L. (2013). Membrane stress caused by octanoic acid in Saccharomyces cerevisiae. Applied Microbiology and Biotechnology, 97(7), 3239-3251. https://doi.org/10.1007/s00253-0134773-5

Sambrook, J., Fritsch, E., \& Maniatis, T. (1989). Molecular cloning: A laboratory manual. Second edition. In G. A. Evans (Ed.), Cold Spring Harbor Laboratory Press, New York. (Vol. 61, Issue 1). https://doi.org/10.1016/0092-8674(90)90210-6

Schüller, C., Mamnun, Y. M., Wolfger, H., Rockwell, N., Thorner, J., \& Kuchler, K. (2007). Membrane-active compounds activate the transcription factors Pdr1 and Pdr3 connecting pleiotropic drug resistance and membrane lipid homeostasis in Saccharomyces cerevisiae. Molecular Biology of the Cell, 18(12), 4932-4944. https://doi.org/10.1091/mbc.E07-06-0610

Shi, S., Chen, Y., Siewers, V., \& Nielsen, J. (2014). Improving production of malonyl coenzyme Aderived metabolites by abolishing Snf1-dependent regulation of Acc1. MBio, 5(3), e0113014. https://doi.org/10.1128/mBio.01130-14

Taxis, C., \& Knop, M. (2006). System of centromeric, episomal, and integrative vectors based on drug resistance markers for Saccharomyces cerevisiae. BioTechniques, 40(1), 73-78. https://doi.org/10.2144/000112040

Thakur, J. K., Arthanari, H., Yang, F., Pan, S.-J., Fan, X., Breger, J., Frueh, D. P., Gulshan, K., Li, D. K., Mylonakis, E., et al. (2008). A nuclear receptor-like pathway regulating multidrug resistance in fungi. Nature, 452(7187), 604-609. https://doi.org/10.1038/nature06836

Viegas, C. A., Almeida, P. F., Cavaco, M., \& Sá-Correia, I. (1998). The H(+)-ATPase in the plasma membrane of Saccharomyces cerevisiae is activated during growth latency in octanoic acid-supplemented medium accompanying the decrease in intracellular $\mathrm{pH}$ and cell viability. Applied and Environmental Microbiology, 64(2), 779-783. http://www.ncbi.nlm.nih.gov/pubmed/9464423

Wernig, F., Baumann, L., Boles, E., \& Oreb, M. (2021). Production of octanoic acid in Saccharomyces cerevisiae: Investigation of new precursor supply engineering strategies and intrinsic limitations. Submitted.

Wernig, F., Boles, E., \& Oreb, M. (2019). De novo biosynthesis of 8-hydroxyoctanoic acid via a medium-chain length specific fatty acid synthase and cytochrome P450 in Saccharomyces cerevisiae. Metabolic Engineering Communications, e00111. https://doi.org/10.1016/j.mec.2019.e00111

Zhu, Z., Hu, Y., Teixeira, P. G., Pereira, R., Chen, Y., Siewers, V., \& Nielsen, J. (2020). Multidimensional engineering of Saccharomyces cerevisiae for efficient synthesis of medium-chain fatty acids. Nature Catalysis, 3(1), 64-74. https://doi.org/10.1038/s41929019-0409-1 


\section{Publications}

\subsection{A yeast-based biosensor for screening of short- and medium-chain fatty acid production}

Declaration of author contributions to the publication:

A yeast-based biosensor for screening of short- and medium-chain fatty acid production

Status: published, October 2018

Journal: ACS Synthetic Biology

Type of publication: research article

Contributing authors: Leonie Baumann (LB), Arun S. Rajkumar (AR), John P.

Morrissey (JM), Eckhard Boles (EB) \& Mislav Oreb (MO)

Contributions of doctoral candidate and co-authors

(1) Concept and design

Doctoral candidate LB: $65 \%$

Co-authors AR, EB, MO: 10\%, 10\%, 15\%

(2) Conducting tests and experiments

Doctoral candidate LB: $100 \%$, yeast molecular biology, biosensor and growth measurements, fermentations, metabolite extraction and GC analysis

(3) Compilation of data sets and figures

Doctoral candidate LB: $100 \%$, biosensor assays, metabolite titers

(4) Analysis and interpretation of data

Doctoral candidate LB: $70 \%$, biosensor assays, metabolite titers

Co-authors EB, MO: $10 \%, 20 \%$, supervision and advice

(5) Drafting of manuscript

Doctoral candidate LB: $80 \%$

Co-authors JM, EB, MO: 5\%, 5\%, 10\% 
SyCStheticBiology

\title{
A Yeast-Based Biosensor for Screening of Short- and Medium-Chain Fatty Acid Production
}

\author{
Leonie Baumann, ${ }^{\dagger}$ Arun S. Rajkumar, ${ }^{\ddagger}$ John P. Morrissey, ${ }^{\ddagger}$ Eckhard Boles, ${ }^{\dagger}$ and Mislav Oreb ${ }^{*}, \dagger$ \\ ${ }^{\dagger}$ Institute of Molecular Biosciences, Faculty of Biological Sciences, Goethe University Frankfurt, Max-von-Laue Straße 9, 60438 \\ Frankfurt am Main, Germany \\ ${ }^{*}$ School of Microbiology, Centre for Synthetic Biology and Biotechnology, Environmental Research Institute, APC Microbiome \\ Institute, University College Cork, Cork T12 YN60, Ireland
}

Supporting Information

\begin{abstract}
Short- and medium-chain fatty acids (SMCFA) are important platform chemicals currently produced from nonsustainable resources. The engineering of microbial cells to produce SMCFA, however, lacks high-throughput methods to screen for best performing cells. Here, we present the development of a whole-cell biosensor for easy and rapid detection of SMCFA. The biosensor is based on a multicopy yeast plasmid containing the SMCFA-responsive PDR12 promoter coupled to GFP as the reporter gene. The sensor detected hexanoic, heptanoic and octanoic acid over a linear range up to $2,1.5$, and $0.75 \mathrm{mM}$, respectively, but did not show a linear response to decanoic and dodecanoic acid. We validated the functionality of the biosensor with culture supernatants of a previously engineered Saccharomyces cerevisiae octanoic acid producer strain and derivatives thereof. The biosensor signal correlated strongly with the octanoic acid concentrations as determined by gas chromatography. Thus, this biosensor enables the high-throughput screening of SMCFA producers and has the potential to drastically speed up the engineering of diverse SMCFA producing cell factories.

KEYWORDS: biosensor, high-throughput screening, short-chain fatty acids, medium-chain fatty acids, octanoic acid, PDR12
\end{abstract}

S hort- and medium-chain fatty acids (SMCFA) and their $\checkmark$ derivatives have a wide range of industrial applications such as pharmaceuticals, antimicrobials or biofuels. ${ }^{1}$ Rising concerns about nonsustainable production methods, such as deforestation and competition with the food industry, makes the fermentative production from renewable resources by microbial cell factories increasingly attractive. In recent years, several reports about the successful engineering of strains for the production of SMCFA have been published, but yields, titers and productivities remain low. ${ }^{2}$ One of the reasons for this is the lack of high-throughput screening methods for randomly mutated or rationally engineered strains to identify highly producing cells, as the common screening methods currently applied are mostly based on low-throughput gas (GC) or liquid chromatography (LC). ${ }^{3}$

The use of biosensors for screening has become a popular alternative to chromatography and can substantially accelerate the optimization of production strains. ${ }^{4}$ Most developed biosensor systems rely on the expression of sensing elements, e.g., genes encoding extracellular receptors, under the control of an inducible promoter, and are coupled with an easily detectable output such as fluorescence, luminescence, colorimetry or growth rate. ${ }^{4}$

To our knowledge only one whole-cell SMCFA biosensor was developed so far, which was also based on this principle. It made use of heterologous G-protein coupled receptors
(GPCRs) in combination with the yeast mating pathway for signaling and a reporter construct inducing GFP expression upon exposure to octanoic acid $\left(\mathrm{C}_{8} \mathrm{FA}\right)$ and decanoic acid $\left(\mathrm{C}_{10} \mathrm{FA}\right)$. Although this GPCR-based sensor system has the advantage of being amenable to the sensing of other compounds by exchanging the receptor unit, ${ }^{6}$ it has some drawbacks including complicated strain construction (several gene deletions and insertions), the need for functional expression of heterologous plasma membrane localized receptors as well as a low linear range for $\mathrm{C}_{8} \mathrm{FA}$ detection. Most importantly, the functionality of this sensor could not be verified in a mixture with other secreted acids, i.e., myristic and palmitic acid $\left(\mathrm{C}_{14}\right.$ and $\left.\mathrm{C}_{16} \mathrm{FA}\right)$, which was partially attributed to the toxicity of FA on cell growth. ${ }^{5}$ However, functionality of a sensor in a mixture of different acids, as found in culture supernatants, would be essential for practical application.

Due to these limitations, we assumed that a promoter-based biosensor for SMCFA detection could be a more promising approach. There are several examples of transcription factor/ promoter-based biosensors developed for the detection of environmental pollutants but not so many for the screening of mutant libraries to identify best performing cells. ${ }^{711} \mathrm{~A}$ prerequisite for the use of such systems as biosensors is the

Received: July 20, 2018

Published: October 19, 2018 
identification of a substrate-specific transcription factor and its respective promoter. ${ }^{12}$ The PDR12 promoter appeared as a suitable candidate for the sensor system that we envisioned, as it is rapidly activated by only one transcription factor ${ }^{13,14}$ and responds to a narrow spectrum of substrates. ${ }^{13,15-17}$ PDR12 encodes an ATP-binding cassette transporter and is under control of the $\mathrm{Zn}_{2} \mathrm{Cys}_{6}$ transcription factor Warlp. ${ }^{13,14}$ Warlp constitutively binds to a weak acid response element (WARE) in the PDR12 promoter. Upon weak acid stress, Warlp is phosphorylated and undergoes conformational changes leading to its activation and thereby PDR 12 expression. ${ }^{14,18,19}$ PDR 12 has been shown so far to be induced by multiple substrates, i.e., propanoic, butanoic, pentanoic, hexanoic and heptanoic acids $\left(\mathrm{C}_{3}-\mathrm{C}_{7} \mathrm{FA}\right), \mathrm{C}_{8} \mathrm{FA},{ }^{15,17,20}$ sorbate and benzoate ${ }^{13}$ but not by acetate, ${ }^{16}$ succinate, citrate ${ }^{17}$ or organic alcohols. ${ }^{15}$ The role of $\mathrm{Pdr} 12 \mathrm{p}$ in response to $\mathrm{C}_{3}-\mathrm{C}_{8}$ FA has been demonstrated with a $\Delta p d r 12$ mutant that was hypersensitive and the activation of a PDR12-lacZ reporter construct in response to these FA..$^{15,17}$ Furthermore, a microarray analysis revealed the activation of PDR12 expression upon exposure of yeast cells to $\mathrm{C}_{8} \mathrm{FA} .{ }^{20} \mathrm{In}$ addition to these characteristics, the PDR12 promoter has a low constitutive activity in the absence of stress, ${ }^{14}$ but upon the appropriate signal exposure, Pdr12p becomes one of the most abundant plasma membrane proteins. ${ }^{21}$

These previous findings led us to the assumption that GFP expression driven by the PDR12 promoter could be suitable as a biosensor for SMCFA. We further sought to develop it as a two-cell sensor system in which SMCFA sensing is decoupled from SMCFA production for several reasons. First, with increasing titers, the producer microbe supernatant can simply be diluted to stay in the linear range of the sensor, which also helps to evade detrimental effects of SMCFA on yeast growth at higher concentrations. ${ }^{22} \mathrm{~A}$ one-cell sensor system could lead to false-negatives due to a saturation of the sensor signal at concentrations exceeding the linear range. Second, the producer microbe can be further engineered or modified without affecting the sensor function. Finally, we want to select for cells producing high extracellular titers. In a one-cell system, an intracellular sensor will only measure the cytosolic amount of produced SMCFA, which can lead, for example, to the identification of transport-deficient mutants that accumulate SMCFA intracellularly instead of producing higher titers.

Previously, we engineered an $S$. cerevisiae strain producing mainly $\mathrm{C}_{8}$ FA with titers of up to $245 \mathrm{mg} / \mathrm{L}(1.7 \mathrm{mM})$ and minor amounts of $\mathrm{C}_{6}, \mathrm{C}_{10}$ and $\mathrm{C}_{12}$ FA. $^{23}$ Interested in improving this strain for higher titers, we aimed at developing a biosensor assay for rapid detection of SMCFA in culture supernatants of this strain and mutants thereof.

\section{RESULTS AND DISCUSSION}

Design of a PDR12 Promoter-Based Biosensor for SMCFA. We sought to develop a two-cell sensor system in which SMCFA sensing is decoupled from SMCFA production as schematically illustrated in Figure 1. The biosensor was designed by fusing the PDR12 promoter (corresponding to 1168 bp upstream of the PDR12 coding sequence) ${ }^{15}$ to the open reading frame of enhanced GFP, namely Envy, ${ }^{24}$ and inserted into a multicopy plasmid that was transformed into S. cerevisiae CEN.PK113-11C. The sensor's functionality relies on the uptake of exogenous SMCFA, such as $\mathrm{C}_{8} \mathrm{FA}$, by the yeast cells. ${ }^{25}$ At physiological $\mathrm{pH}, \mathrm{C}_{8}$ FA are taken up via active transport, whereas at acidic $\mathrm{pH}$ the undissociated acid form can enter the cell by passive diffusion. ${ }^{22,26}$ Once taken up by

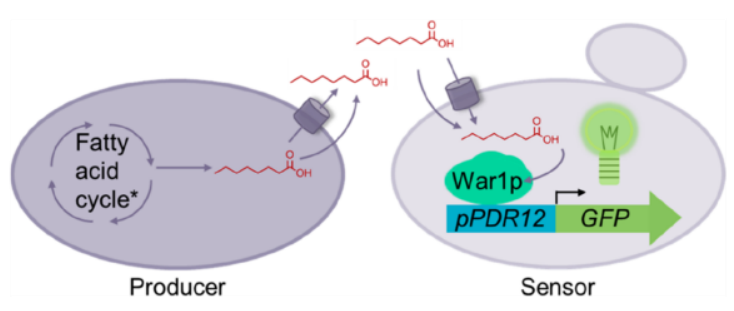

Figure 1. Short- and medium-chain fatty acid detection by a wholecell biosensor. A producer microbe secretes short- and/or mediumchain fatty acids (SMCFA), such as octanoic acid shown here, which are produced through a modified fatty acid cycle $(*)$. The culture supernatant is added to the yeast biosensor strain, which takes up the SMCFA via diffusion or active transport. The biosensor consists of the PDR12 promoter ( $p$ PDR12) coupled to an enhanced version of green fluorescent protein (GFP). The transcription factor Warlp, which is constitutively bound to $P$ PDR 12 , undergoes phosphorylation and conformational changes upon signal exposure and initiates the expression of GFP.

the cell, the $\mathrm{C}_{8} \mathrm{FA}$ activates Warlp, leading to transcription from the PDR12 promoter. We found that GFP expression is activated only in the presence of the inducing signal (here: $\mathrm{C}_{8}$ FA); in the absence of signal, we observed only low constitutive fluorescence (Figure S1). To prove the functionality of the biosensor in culture supernatants, we used a previously constructed S. cerevisiae strain (RPY21/FAS ${ }^{\mathrm{R} 1834 \mathrm{~K}}$ ) that produces mainly $\mathrm{C}_{8} \mathrm{FA}$, which is secreted from or diffuses out of the cells. ${ }^{25}$

Characterization and Verification of Functionality of the Biosensor. We analyzed the biosensor response to $\mathrm{C}_{8} \mathrm{FA}$ concentrations between 0 and $1 \mathrm{mM}$ for an initial validation in defined medium (SCD). We observed a correlation of the RFI (relative fluorescence intensity) of the biosensor to $\mathrm{C}_{8} \mathrm{FA}$ over the entire concentration range and a linear response between 0.01 and $0.75 \mathrm{mM}$ (Figure $2 \mathrm{~A}$ ), which is a strong improvement over a previously published GPCR-based $\mathrm{C}_{8}$ FA sensor (0.019-0.25 mM linear range). The highest intensity of the signal after activation showed a nearly 10 -fold increase after $2 \mathrm{~h}$ of incubation with $\mathrm{C}_{8} \mathrm{FA}$, displaying a high dynamic range (Figure 2A, Figure S2). To make sure that the activation of the biosensor is specific, we tested a control strain with a plasmid expressing GFP under control of the MET25 promoter, which is methionine repressible. In this control strain we observed no $\mathrm{C}_{8}$ FA concentration-dependent induction of GFP expression in SCD medium without methionine (Figure 2A).

Industrial microbial fermentations commonly use complex medium rather than defined medium. Therefore, we next tested the biosensor in a complex medium, i.e., YPD. Again, we observed a correlation of the RFI to the tested $\mathrm{C}_{8}$ FA concentrations $(0-3 \mathrm{mM})$ and an identical linear range between 0.01 and $0.75 \mathrm{mM}$ (Figure 2B, Figure S3). While the dynamic range of the sensor was lower than in SCD medium even after twice the incubation time (Figure S4), this can be attributed to the higher background fluorescence of YPD. Nevertheless, the maximum increase in the intensity of the signal after activation was more than tripled after $4 \mathrm{~h}$ of incubation. Even though there is a discrepancy in previous reports about the PDR12 induction by $\mathrm{C}_{8} \mathrm{FA},{ }^{15,17,20}$ we could clearly show that the PDR12 promoter's $\mathrm{C}_{8}$ FA-dependent response allows it to be used as a biosensor. To our knowledge, this is the first SMCFA biosensor shown to function reliably in 
A

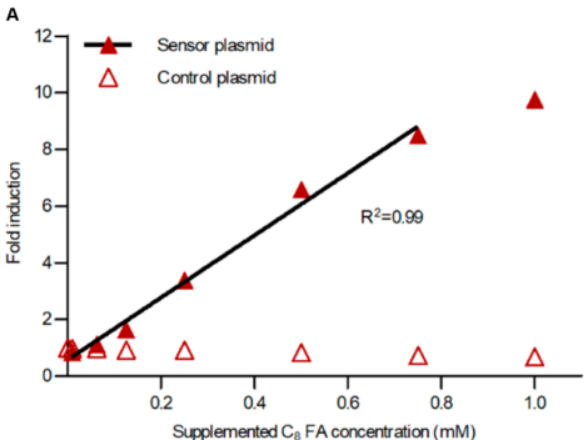

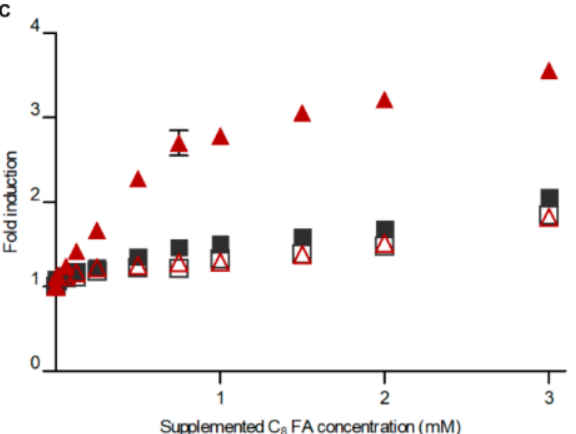

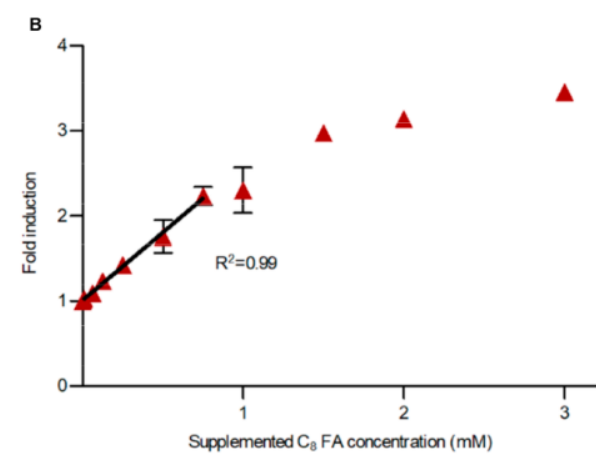

- Sensor plasmid

$\triangle$ Control plasmid

- Sensor genomic

Control genomic

Supplemented $\mathrm{C}_{\mathrm{s}} \mathrm{FA}$ concentration (mM)

Figure 2. $\mathrm{C}_{8}$ fatty acid-dependent response of plasmid-based and genomically integrated biosensors. Response and linear range of the plasmidbased biosensor after $2 \mathrm{~h}$ incubation with $\mathrm{C}_{8}$ fatty acids (FA) in SCD (A) and after $4 \mathrm{~h}$ in YPD medium (B). Error bars represent two technical replicates. Experiments were conducted in three biological replicates with comparable results (Figure S2, Figure S3). (C) Response of the genomically integrated biosensor after $4 \mathrm{~h}$ incubation with $\mathrm{C}_{8}$ FA in YPD. Sensor plasmid: CEN.PK113-11C + p426pPDR12-GFP. Control plasmid: CEN.PK113-11C + p426pMET25-GFP. Sensor genomic: LBY27. Control genomic: CEN.PK113-11C. For fold induction, fluorescence intensities $(\mathrm{FI})$ were divided by optical densities $\left(\mathrm{OD}_{600}\right)$ and normalized to $\mathrm{FI} / \mathrm{OD}_{600}$ values of samples without $\mathrm{C}_{8} \mathrm{FA}$.

complex medium. This is of particular importance since the highest SMCFA titers produced in yeast so far were reached in complex medium. ${ }^{23,27,28}$

To avoid possible stability problems by using a multicopy plasmid-based biosensor, we integrated the sensor construct into the $S$. cerevisiae CEN.PK113-11C genome resulting in strain LBY27. This sensor strain, however, only showed a barely detectable increase in the intensity of the signal upon $\mathrm{C}_{8}$ FA addition in comparison to the control CEN.PK113-11C strain (Figure 2C). Therefore, a multicopy plasmid seems to be necessary for a good dynamic range, and we continued to work with the plasmid-based biosensor.

The Biosensor Also Responds to $C_{6}$ and $C_{7}$ but Not $\mathrm{C}_{10}$ or $\mathrm{C}_{12}$ FA. It was shown in previous work that PDR12 expression is influenced by several weak acids. ${ }^{13,15-17}$ As none of these compounds-except other SMCFA-are usually produced by $S$. cerevisiae, we reasoned that they should not influence the biosensor's ability to detect SMCFA in $S$. cerevisiae culture supernatants.

The previously engineered $S$. cerevisiae strain RPY21/ FAS $^{\mathrm{R} 1834 \mathrm{~K}}$ mainly produces $\mathrm{C}_{8} \mathrm{FA}$ and in minor amounts $\mathrm{C}_{6}$, $\mathrm{C}_{10}$ and $\mathrm{C}_{12}$ FA. To test whether the biosensor also detects these byproducts, we analyzed its response upon addition of $\mathrm{C}_{6}, \mathrm{C}_{10}$ and $\mathrm{C}_{12}$ FA to YPD. As depicted in Figure 3, the RFI of the biosensor correlates to $\mathrm{C}_{6}$ FA levels between 0 and $3 \mathrm{mM}$, and the biosensor showed a linear response between 0.01 and $2 \mathrm{mM}$ in all replicates (Figure S3). $\mathrm{C}_{10}$ FA seems to be very toxic to the cells, as growth is already strongly decreased at the

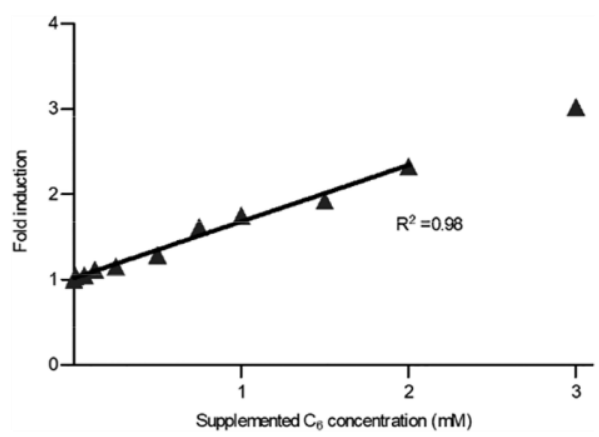

Figure 3. $\mathrm{C}_{6}$ fatty acid-dependent response of the biosensor Response and linear range of the biosensor after $4 \mathrm{~h}$ incubation with $\mathrm{C}_{6}$ fatty acids (FA) in YPD medium. Error bars represent two technical replicates. Experiments were conducted in three biological replicates with comparable results (Figure S3). For fold induction, fluorescence intensities (FI) were divided by optical densities $\left(\mathrm{OD}_{600}\right)$ and normalized to $\mathrm{FI} / \mathrm{OD}_{600}$ values of samples without FA.

lower concentrations, which leads to false high inductions when dividing FI by $\mathrm{OD}_{600}$ values (Figure S3). This is in accordance with previous findings about high toxicity of $\mathrm{C}_{10}$ FA. ${ }^{22}$ Overall, we cannot exclude a very slight response of the biosensor to $\mathrm{C}_{10} \mathrm{FA}$ at low concentrations $(0.01-0.25 \mathrm{mM})$, which would be in accordance to a previous report suggesting a discrete role of Pdr12p in $\mathrm{C}_{10}$ FA response. ${ }^{17}$ Nevertheless, we 
A

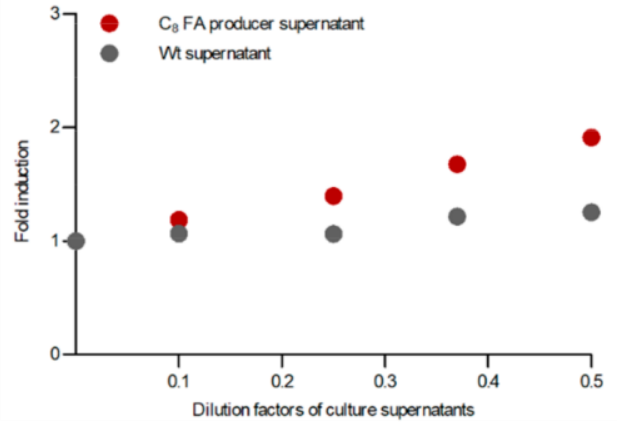

B

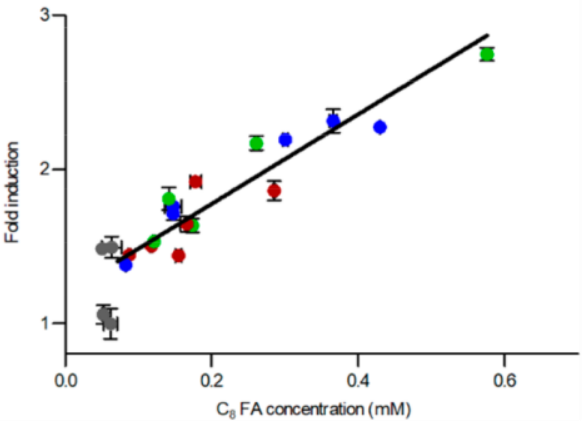

Figure 4. Biosensor response to $\mathrm{C}_{8}$ fatty acids in $S$. cerevisiae culture supernatants and correlation to GC measurement. (A) Response of the biosensor to differently diluted culture supernatants of a $\mathrm{C}_{8}$ fatty acid (FA) producer (RPY21/FAS $\left.\mathrm{R}^{\mathrm{R} 1834 \mathrm{~K}} / \mathrm{pRS} 42 \mathrm{H}\right)$ and a wildtype strain (Wt, CEN.PK113-7D). (B) Linear correlation of the fold induction of biosensor signal in 0.5 dilutions of culture supernatants with GC measurements of the same supernatants. Strains: CEN.PK113-7D (gray), RPY21/FAS ${ }^{\text {R1834K } / p R S 42 H ~(r e d), ~ R P Y 21 / F A S ~}{ }^{\text {R1834K } / L B V 17 ~(b l u e), ~ R P Y 21 / ~}$ $\mathrm{FAS}^{\mathrm{R} 1834 \mathrm{~K}} / \mathrm{LBV} 20$ (green).

could not observe a linear response range of the biosensor to $\mathrm{C}_{10} \mathrm{FA}$ in all three biological replicates.

For $\mathrm{C}_{12} \mathrm{FA}$, we found a concentration-dependent decrease in fluorescence over the entire concentration range tested and an increase in the $\mathrm{OD}_{600}$ at $\mathrm{C}_{12} \mathrm{FA}$ concentrations between 0.25 and $1 \mathrm{mM}$ (Figure S5). We also observed this when adding $\mathrm{C}_{12}$ FA to plain medium not containing the biosensor strain (data not shown). Therefore, this is probably due to incomplete solubility and light scattering by $\mathrm{C}_{12}$ FA micelles. In addition, we also tested the biosensor response to the odd chain $\mathrm{C}_{7} \mathrm{FA}$ and observed a linear response between 0.01 and $1.5 \mathrm{mM}$ (Figure S6). This is in accordance to previous studies reporting that PDR12 is induced by $\mathrm{C}_{7}$ FA. ${ }^{15,17}$

In summary, we show that the biosensor responds to $\mathrm{C}_{6}, \mathrm{C}_{7}$ and $\mathrm{C}_{8} \mathrm{FA}$ in YPD medium over linear ranges from 0.01 up to $2,1.5$, and $0.75 \mathrm{mM}$, respectively, whereas it does not show a linear response to $\mathrm{C}_{10} \mathrm{FA}$ and no concentration-dependent response at all to $\mathrm{C}_{12} \mathrm{FA}$.

Detection of $C_{8} F A$ by the Biosensor in S. cerevisiae Culture Supernatants. To validate that the biosensor can discriminate between different SMCFA concentrations in culture supernatants, we first conducted a pretest. We cultivated a $\mathrm{C}_{8}$ FA producer strain (RPY21/FAS $\mathrm{F}^{\mathrm{R} 1834 \mathrm{~K}}$ / pRS42H) and a wildtype strain (CEN.PK113-7D) in buffered YPD (with hygromycin if applicable) for $72 \mathrm{~h}$ and added the culture supernatants in different dilutions to the biosensor, and in parallel we measured the $\mathrm{C}_{8} \mathrm{FA}$ content by GC. As shown in Figure $4 \mathrm{~A}$, the lowest dilution $(0.5)$ of the $\mathrm{C}_{8} \mathrm{FA}$ producer supernatant led to a 1.9-fold increase in the intensity of the signal after $4 \mathrm{~h}$ of incubation. Furthermore, we observed a constant increase in signal intensity with decreasing dilution factors confirming the $\mathrm{C}_{8} \quad \mathrm{FA}$ concentration-dependent activation of the biosensor in S. cerevisiae culture supernatants. The supernatant of the wildtype culture also led to a slight increase in signal intensity of the biosensor, which can be attributed to minor amounts of $\mathrm{C}_{8} \mathrm{FA}$ that it produced naturally. As determined by GC, the wildtype strain produced $0.02 \mathrm{mM} \mathrm{C}_{8} \mathrm{FA}$, whereas $\mathrm{RPY} 21 / \mathrm{FAS}^{\mathrm{R} 1834 \mathrm{~K}} / \mathrm{pRS} 42 \mathrm{H}$ produced $0.28 \mathrm{mM} \mathrm{C}_{8} \mathrm{FA}$.

To obtain supernatants with a wide range of $\mathrm{C}_{8} \mathrm{FA}$ concentrations we not only tested the original RPY21/ $\mathrm{FAS}^{\mathrm{R} 1834 \mathrm{~K}}$ strain but also two derivatives thereof. We transformed the strain with one of two plasmids containing overexpressed genes or the control plasmid (pRS42H), respectively. One plasmid harbored an overexpression construct of TPO1 (LBV17). The transporter Tpolp seems to be involved in the resistance to $\mathrm{C}_{8} \mathrm{FA}^{20}$ and therefore has the potential to decrease $\mathrm{C}_{8} \mathrm{FA}$ toxicity. The second construct encoded the acetyl-CoA carboxylase ACC1 and harbored mutations leading to amino acid exchanges of two serine residues to alanine $\left(A C C 1^{\text {S659AS1157A; }}\right.$ LBV20). These two mutations impair the deactivation of Acclp via phosphorylation. ${ }^{29-31}$ A similarly modified Acclp $\left(A C C 1^{S 1157 A}\right)$ was shown to have a positive effect on yeast resistance to $\mathrm{C}_{8} \mathrm{FA},{ }^{32}$ and therefore we reasoned that it could also influence $\mathrm{C}_{8}$ FA product titers of RPY21/FAS ${ }^{\mathrm{R} 1834 \mathrm{~K}}$. We cultivated RPY21/ $\mathrm{FAS}^{\mathrm{R} 1834 \mathrm{~K}}$ with overexpression or control plasmids as well as a wildtype strain (CEN.PK113-7D) in two independent experiments in two to three replicates and obtained a wide range of $\mathrm{C}_{8} \mathrm{FA}$ titers between 0.05 and $0.58 \mathrm{mM}$ as measured by GC. We added the culture supernatants to the biosensor (corresponding to a 0.5 dilution) and measured the RFI. As shown in Figure 4B, the RFI of the biosensor clearly correlates with the $\mathrm{C}_{8}$ FA concentrations measured by GC. A linear correlation could be observed for concentrations of produced $\mathrm{C}_{8} \mathrm{FA}$ from $0.08 \mathrm{mM}$ up to the highest concentration tested, $0.58 \mathrm{mM}$. We additionally tested the supernatants in a dilution of 0.25 with the biosensor and received-despite lower fold inductions-an identical linear range (Figure S7A). In all samples, the $\mathrm{C}_{6}$ FA content was below $6 \%$ of the total SMCFA titers and did not alter the linear correlation of the biosensor response to $\mathrm{C}_{8}$ FA concentrations (Figure S7B).

This is the first report of a whole-cell promoter-based biosensor for the detection of SMCFA in culture supernatants. In contrast to previously generated sensor systems, ${ }^{5,6,8-10}$ the here presented biosensor solely consists of an S. cerevisiae strain transformed with a multicopy plasmid containing the PDR12 promoter coupled to GFP without further strain engineering, fine-tuning or expression of additional components needed. We demonstrate that it linearly responds to $\mathrm{C}_{8} \mathrm{FA}$ in defined and complex medium as well as to $\mathrm{C}_{6}$ and $\mathrm{C}_{7} \mathrm{FA}$ in complex medium. Furthermore, the biosensor responds to $\mathrm{C}_{8} \mathrm{FA}$ concentrations from 0.08 up to at least $0.58 \mathrm{mM}$ in S. cerevisiae culture supernatants and therefore in a mixture of other secreted FA. The biosensor response showed a clear correlation of RFI to $\mathrm{C}_{8} \mathrm{FA}$ values determined by GC and 
therefore can be used to compare SMCFA titers of different culture supernatants. On the basis of these results, we envision two possible applications. It can be used as a screening system of microbial producer strain libraries of one specific SMCFA, as shown for the $\mathrm{C}_{8} \mathrm{FA}$ producing strain here, or to screen for increased overall SMCFA titers. For the latter, GC measurements can be used to determine the exact SMCFA composition of the culture supernatants once a biosensoraided screening resulted in several good performing candidate strains.

The currently produced amounts of $\mathrm{C}_{6}$ FA by yeast vary from 0.17 to $0.62 \mathrm{mM}^{23,28,33}$ and fall within the linear range of the biosensor. On the other hand, $\mathrm{C}_{8} \mathrm{FA}$ titers produced by yeast vary from 0.45 to $1.7 \mathrm{mM}^{23,28,33}$ and therefore partly exceed the linear range of the biosensor. However, we can simply dilute culture supernatants before adding them to the biosensor to retain a linear response. The biosensor also has a wide linear range for $\mathrm{C}_{7} \mathrm{FA}$ detection and could be a valuable tool to screen a $\mathrm{C}_{7}$ FA-producer library; however, yeast has not been engineered to produce $\mathrm{C}_{7} \mathrm{FA}$ so far.

For future use, a more stable, plasmid-independent sensor strain might be beneficial. This could be achieved by a multicopy integration of the sensor into the genome. Thereby, the low dynamic range of the sensor response as seen with the single genomic integration might be increased. The dynamic range of the sensor could be further optimized by the genomic replacement of the native WAR1 promoter with the PDR12 promoter. ${ }^{11}$ Assuming low "leaky" expression levels of WAR1, such a positive-feedback loop would accelerate WAR1 expression in the presence of inducing molecules. These higher Warlp levels would then result in a higher expression of PDR12 promoter-coupled GFP, possibly leading to higher dynamic ranges of the sensor. To improve the linear range of the biosensor, the saturation of the sensor signal would have to be shifted toward higher FA concentrations. One possibility to achieve this could be to decrease the binding affinity of Warlp to the WARE site in the PDR12 promoter. This might be accomplished either by mutating WAR1 or the WARE site. Alternatively, Warlp could be mutated to exhibit a lower binding affinity for its inducers; however, it is known neither if the inducing molecules directly bind Warlp nor at which molecular site. ${ }^{14,18,19}$ In order to try any of these approaches, detailed Warlp structural data would be needed.

Nevertheless, the here presented biosensor has the appropriate linear and dynamic ranges to use it for highthroughput screening of yeast SMCFA production. Needing only $4 \mathrm{~h}$ of incubation of sample supernatants with the biosensor in multiwell format, hundreds of strains can be rapidly screened to select the best performing strains. For future use, we envision a broader application spectrum of the biosensor as its use is not restricted per se to S. cerevisiae or even yeast SMCFA producers. The biosensor's SMCFA response certainly will have to be confirmed in the supernatants of other microbes, i.e., other yeasts or bacteria, as different species also produce different metabolites that could influence the biosensor's response to SMCFA in other culture supernatants. Overall, this biosensor represents a valuable and ready-to-use system, which fills a need to rapidly improve microbial SMCFA production.

\section{METHODS}

Strains and Plasmid Construction. Yeast strains and plasmids used throughout this study are listed in Table S1. The
PDR12 promoter, TPO1 and ACC1 ${ }^{\text {S659AS1157A }}$ as well as promoters ( $p P G K 1, p P Y K 1)$ and endogenous terminators ( $t$ TPO1, $t A C C 1)$ were amplified from CEN.PK113-11C genomic DNA with primers containing the respective overhangs for cloning via homologous recombination (oligonucleotides are listed in Table S2). The PDR12 promoter was integrated into the SacI site of p426pMET25-GFP, thereby replacing $p M E T 25$. The genomic insertion of $p P D R 12-G F P$ was performed via CRISPR/Cas9 as described previously. ${ }^{34}$ The CRISPR/Cas9 plasmid was amplified in two PCR fragments and transformed for in vivo assembly. The insertion fragment was amplified from plasmid p426pPDR12-GFP. Yeast transformations were performed according to Gietz and Schiest ${ }^{35}$ or for RPY21 via an adapted procedure. ${ }^{23}$ To generate RPY21/FAS ${ }^{\mathrm{R} 1834 \mathrm{~K}}$, RPY21 was transformed with a plasmid carrying the wildtype version of FAS2 and another plasmid carrying FAS1 with mutations leading to an amino acid replacement (R1834K), thereby redirecting the FA production from long chain FA to SMCFA. ${ }^{23}$ Cells were streaked out on selective YPD ( $1 \%$ yeast extract, $2 \%$ peptone, both produced by BD, Difco Laboratories, Sparks, USA; 2\% Dglucose, purchased from Roth, Karlsruhe, Germany) containing hygromycin or G418 $(200 \mu \mathrm{g} / \mathrm{mL})$ to select for $h p h N T 1$ or kanMX or on selective SCD medium ${ }^{36}$ lacking leucine, tryptophan, uracil and/or histidine $( \pm \mathrm{LWUH})$ to select for LEU2, URA3 or HIS3 markers, respectively. Electrocompetent E. coli DH10 $\beta$ (Gibco BRL, Gaithersburg, MD) was used for subcloning according to standard procedures, and transformants were selected on lysogeny broth (LB) agar plates ${ }^{37}$ supplemented with $100 \mu \mathrm{g} / \mathrm{mL}$ ampicillin.

Cultures for Fatty Acid Production. $\mathrm{C}_{8}$ FA producer and control (wildtype) strains were grown as previously described $^{23}$ with minor adjustments. For precultures, several colonies of the strains were picked and combined in $20 \mathrm{~mL}$ YPD with $100 \mathrm{mM}$ potassium phosphate buffer ( $\mathrm{pH}$ 6.5). After shaking at 180 r.p.m. at $30^{\circ} \mathrm{C}$ overnight, the main culture was inoculated to an $\mathrm{OD}_{600}$ of 0.1 in $30 \mathrm{~mL}$ buffered YPD medium and cultured in $300 \mathrm{~mL}$ shake flasks under the same conditions. After $72 \mathrm{~h}$, the cultures were harvested by centrifugation and $20 \mathrm{~mL}$ of the supernatant was used for FA extraction (see below) whereas remaining supernatant was used for the biosensor assay.

Fatty Acid Extraction and Quantification via GC. FA extraction and GC analysis were performed as described previously. ${ }^{25}$ For each culture, two $10 \mathrm{~mL}$ aliquots from the same culture supernatant were separately processed and measured by GC. The standard deviation (SD) between the two measurements from the same culture was for all samples below $2 \mathrm{mg} / \mathrm{L}$.

Cultures for the Biosensor Assay. For the biosensor assay, strains (CEN.PK113-11C/p426pMET25-GFP or p426pPDR12-GFP) were grown overnight with 180 r.p.m. shaking at $30{ }^{\circ} \mathrm{C}$ in SCD-L-W-U and under addition of repressive concentrations of methionine $(2 \mathrm{mM})$ for the strain harboring plasmid p426pMET25-GFP. Precultured cells were used to inoculate $20 \mathrm{~mL}$ of fresh SCD-L-W-U (+2 mM methionine) to an $\mathrm{OD}_{600}$ of 0.1 and grown for $4-6 \mathrm{~h}$ until an $\mathrm{OD}_{600}$ of about 0.4. Cells were centrifuged and resuspended in fresh YPD or SCD-L-W-U without methionine and transferred into black 96-well plates with clear flat bottom (ref. 655097, Greiner Bio-one, Frickenhausen, Germany) with $100 \mu \mathrm{L}$ per well. To keep conditions sterile, well plates were sealed with a gas permeable sealing membrane (Breathe-Easy, Diversified 
Biotech, Dedham, MA, USA). For the primary verification of the sensor, $90 \mu \mathrm{L}$ of fresh media was added together with 10 $\mu \mathrm{L}$ of $70 \% \mathrm{v} / \mathrm{v}$ ethanol (control) or $10 \mu \mathrm{L}$ of differently diluted fatty acids (in $70 \% \mathrm{v} / \mathrm{v}$ ethanol) to always reach a final volume of $200 \mu \mathrm{L} /$ well and have the same ethanol concentrations in all wells. $\mathrm{C}_{6}, \mathrm{C}_{7}, \mathrm{C}_{8}, \mathrm{C}_{10}$ and $\mathrm{C}_{12} \mathrm{FA}$ (Sigma-Aldrich) were diluted in $70 \% \mathrm{v} / \mathrm{v}$ ethanol in concentrations between 0.2 and $60 \mathrm{mM}$ to reach final dilutions of 0.01 to $3 \mathrm{mM}$ in the wells. For the biosensor test with culture supernatants, $100 \mu \mathrm{L}$ of culture supernatant (for cultivation conditions see above) was added to $100 \mu \mathrm{L}$ of the biosensor strain. Experiments were performed in biological triplicates with technical duplicates.

Biosensor Measurements. Directly after pipetting of all components into the 96-well plate, it was incubated at $30^{\circ} \mathrm{C}$ and 600 r.p.m. shaking in a CLARIOstar plate reader (BMG Labtech, Ortenberg, Germany). Measurements of $\mathrm{OD}_{600}$ and FI with excitation at $470 \pm 15 \mathrm{~nm}$ and emission at $515 \pm 20$ $\mathrm{nm}$ were taken every $30 \mathrm{~min}$ over several hours. To normalize for different cell numbers in wells, the FI value of each well was divided by the respective $\mathrm{OD}_{600}$ value. For calculating the fold induction, these $\mathrm{FI} / \mathrm{OD}_{600}$ values were divided for the different FA concentrations by the FI/OD 600 values of the " $0 \mathrm{mM}$ " controls (without FA). For calculation of the fold induction response to the culture supernatants, $\mathrm{FI} / \mathrm{OD}_{600}$ values of the wells with supernatant and biosensor were divided by FI/ $\mathrm{OD}_{600}$ values only containing biosensor. Values are shown as mean \pm the standard deviation (SD).

Data analyses including the calculation of linear regression and $R^{2}$ values were performed using the software Prism 5 (GraphPad, USA).

\section{- ASSOCIATED CONTENT}

\section{S Supporting Information}

The Supporting Information is available free of charge on the ACS Publications website at DOI: 10.1021/acssynbio.8b00309.

\section{Supporting figures and tables (PDF)}

\section{- AUTHOR INFORMATION}

\section{Corresponding Author}

*E-mail: m.oreb@bio.uni-frankfurt.de.

\section{ORCID}

Leonie Baumann: 0000-0001-7579-6777

\section{Author Contributions}

LB, EB and MO conceived the study. ASR and JPM provided ideas and constructive input. LB performed all experiments. $\mathrm{LB}, \mathrm{EB}$ and MO analyzed the data and wrote the paper. All authors read and approved the manuscript.

\section{Notes}

The authors declare the following competing financial interest(s): EB is inventor of EP patent application No. 15 162192.7 filed on April 1, 2015, and of EP patent application No. 15174342.4 filed on June 26, 2015, by Goethe-University Frankfurt, concerning short-chain acyl-CoA producing FAS variants. There are no other competing interests.

\section{ACKNOWLEDGMENTS}

The authors thank Joanna Tripp for plasmid p426pMET25GFP. We thank Michael K. Jensen and Jay D. Keasling for providing advice and resources. This project has received funding from the European Union's Horizon 2020 research and innovation program under Grant Agreement No. 720824.

\section{ABBREVIATIONS}

SMCFA, short- and medium-chain fatty acid(s); FA, fatty $\operatorname{acid}(\mathrm{s}) ; \mathrm{C}_{6}, \mathrm{C}_{7}, \mathrm{C}_{8}, \mathrm{C}_{10}, \mathrm{C}_{12} \mathrm{FA}$, hexanoic, heptanoic, octanoic, decanoic, dodecanoic acid(s); FAS, fatty acid synthase; PDR12, plasma membrane ATP-binding cassette transporter; TPO1, polyamine transporter of the major facilitator superfamily; ACC1, acetyl-CoA carboxylase; GFP, green fluorescent protein; GC, gas chromatography; $\mathrm{OD}_{600}$, optical density at $\lambda$ $=600 \mathrm{~nm}$; FI, fluorescence intensity; RFI, relative fluorescence intensity.

\section{REFERENCES}

(1) Sarria, S., Kruyer, N. S., and Peralta-Yahya, P. (2017) Microbial synthesis of medium-chain chemicals from renewables. Nat. Biotechnol. 35, 1158-1166.

(2) Fernandez-Moya, R., and Da Silva, N. A. (2017) Engineering Saccharomyces cerevisiae for High-Level Synthesis of Fatty Acids and Derived Products. FEMS Yeast Res., DOI: 10.1093/femsyr/fox071.

(3) Woolston, B. M., Edgar, S., and Stephanopoulos, G. (2013) Metabolic Engineering: Past and Future. Annu. Rev. Chem. Biomol. Eng. 4, 259-288.

(4) Adeniran, A., Sherer, M., and Tyo, K. E. J. (2014) Yeast-based biosensors: design and applications. FEMS Yeast Res. 15, 1-15.

(5) Mukherjee, K., Bhattacharyya, S., and Peralta-Yahya, P. (2015) GPCR-Based Chemical Biosensors for Medium-Chain Fatty Acids. ACS Synth. Biol. 4, 1261-1269.

(6) Ehrenworth, A. M., Claiborne, T., and Peralta-Yahya, P. (2017) Medium-Throughput Screen of Microbially Produced Serotonin via a G-Protein-Coupled Receptor-Based Sensor. Biochemistry 56, 54715475 .

(7) Dietrich, J. A., McKee, A. E., and Keasling, J. D. (2010) HighThroughput Metabolic Engineering: Advances in Small-Molecule Screening and Selection. Annu. Rev. Biochem. 79, 563-590.

(8) Skjoedt, M. L., Snoek, T., Kildegaard, K. R., Arsovska, D., Eichenberger, M., Goedecke, T. J., Rajkumar, A. S., Zhang, J., Kristensen, M., Lehka, B. J., Siedler, S., Borodina, I., Jensen, M. K., and Keasling, J. D. (2016) Engineering prokaryotic transcriptional activators as metabolite biosensors in yeast. Nat. Chem. Biol. 12, 951958.

(9) Schuller, A., Rödel, G., and Ostermann, K. (2017) Tuning the Sensitivity of the PDR5 Promoter-Based Detection of Diclofenac in Yeast Biosensors. Sensors 17, 1506.

(10) Siedler, S., Khatri, N. K., Zsohár, A., Kjærbølling, I., Vogt, M., Hammar, P., Nielsen, C. F., Marienhagen, J., Sommer, M. O. A., and Joensson, H. N. (2017) Development of a Bacterial Biosensor for Rapid Screening of Yeast $p$-Coumaric Acid Production. ACS Synth. Biol. 6, 1860-1869.

(11) Williams, T. C., Xu, X., Ostrowski, M., Pretorius, I. S., and Paulsen, I. T. (2017) Positive-feedback, ratiometric biosensor expression improves high-throughput metabolite-producer screening efficiency in yeast. Synth. Biol., DOI: 10.1093/synbio/ysw002.

(12) Michener, J. K., Thodey, K., Liang, J. C., and Smolke, C. D. (2012) Applications of genetically-encoded biosensors for the construction and control of biosynthetic pathways. Metab. Eng. 14, 212-222.

(13) Piper, P., Mahé, Y., Thompson, S., Pandjaitan, R., Holyoak, C., Egner, R., Mühlbauer, M., Coote, P., and Kuchler, K. (1998) The pdr12 ABC transporter is required for the development of weak organic acid resistance in yeast. EMBO J. 17, 4257-4265.

(14) Kren, A., Mamnun, Y. M., Bauer, B. E., Schüller, C., Wolfger, H., Hatzixanthis, K., Mollapour, M., Gregori, C., Piper, P., and Kuchler, K. (2003) Warlp, a Novel Transcription Factor Controlling Weak Acid Stress Response in Yeast. Mol. Cell. Biol. 23, 1775-1785. 


\section{Publications}

\section{ACS Synthetic Biology}

(15) Hatzixanthis, K., Mollapour, M., Seymour, I., Bauer, B. E., Krapf, G., Schüller, C., Kuchler, K., and Piper, P. W. (2003) Moderately lipophilic carboxylate compounds are the selective inducers of the Saccharomyces cerevisiae Pdr12p ATP-binding cassette transporter. Yeast 20, 575-585.

(16) Bauer, B. E., Rossington, D., Mollapour, M., Mamnun, Y., Kuchler, K., and Piper, P. W. (2003) Weak organic acid stress inhibits aromatic amino acid uptake by yeast, causing a strong influence of amino acid auxotrophies on the phenotypes of membrane transporter mutants. Eur. J. Biochem. 270, 3189-3195.

(17) Holyoak, C. D., Bracey, D., Piper, P. W., Kuchler, K., and Coote, P. J. (1999) The Saccharomyces cerevisiae weak-acid-inducible $\mathrm{ABC}$ transporter Pdr12 transports fluorescein and preservative anions from the cytosol by an energy-dependent mechanism. J. Bacteriol. 181, 4644-4652.

(18) Gregori, C., Schüller, C., Frohner, I. E., Ammerer, G., and Kuchler, K. (2008) Weak organic acids trigger conformational changes of the yeast transcription factor War1 in vivo to elicit stress adaptation. J. Biol. Chem. 283, 25752-64.

(19) Frohner, I. E., Gregori, C., Anrather, D., Roitinger, E., Schü, C., Ammerer, G., and Kuchler, K. (2010) Weak Organic Acid Stress Triggers Hyperphosphorylation of the Yeast Zinc-Finger Transcription Factor Warl and Dampens Stress Adaptation. OMICS 14, $575-586$

(20) Legras, J. L., Erny, C., Le Jeune, C., Lollier, M., Adolphe, Y., Demuyter, C., Delobel, P., Blondin, B., and Karst, F. (2010) Activation of two different resistance mechanisms in Saccharomyces cerevisiae upon exposure to octanoic and decanoic acids. Appl. Environ. Microbiol. 76, 7526-7535.

(21) Piper, P., Calderon, C. O., Hatzixanthis, K., and Mollapour, M. (2001) Weak acid adaptation: the stress response that confers yeast with resistance to organic acid food preservatives. Microbiology 147, 2635-2642.

(22) Borrull, A., López-Martínez, G., Poblet, M., Cordero-Otero, R. and Rozès, N. (2015) New insights into the toxicity mechanism of octanoic and decanoic acids on Saccharomyces cerevisiae. Yeast 32, $451-460$.

(23) Gajewski, J., Pavlovic, R., Fischer, M., Boles, E., and Grininger, M. (2017) Engineering fungal de novo fatty acid synthesis for short chain fatty acid production. Nat. Commun. 8, 14650.

(24) Slubowski, C. J., Funk, A. D., Roesner, J. M., Paulissen, S. M., and Huang, L. S. (2015) Plasmids for C-terminal tagging in Saccharomyces cerevisiae that contain improved GFP proteins, Envy and Ivy. Yeast 32, 379-387.

(25) Henritzi, S., Fischer, M., Grininger, M., Oreb, M., and Boles, E. (2018) An engineered fatty acid synthase combined with a carboxylic acid reductase enables de novo production of 1-octanol in Saccharomyces cerevisiae. Biotechnol. Biofuels 11, 150.

(26) Viegas, C. A., and Correia, I. S. (1997) Effects of low temperatures $\left(9-33^{\circ} \mathrm{C}\right)$ and $\mathrm{pH}(3.3-5.7)$ in the loss of Saccharomyces cerevisiae viability by combining lethal concentrations of ethanol with octanoic and decanoic acids. Int. J. Food Microbiol. 34, 267-277.

(27) Leber, C., and Da Silva, N. A. (2014) Engineering of Saccharomyces cerevisiae for the synthesis of short chain fatty acids. Biotechnol. Bioeng. 111, 347-358.

(28) Leber, C., Choi, J. W., Polson, B., and Da Silva, N. A. (2016) Disrupted short chain specific $\beta$-oxidation and improved synthase expression increase synthesis of short chain fatty acids in Saccharomyces cerevisiae. Biotechnol. Bioeng. 113, 895-900.

(29) Choi, J. W., and Da Silva, N. A. (2014) Improving polyketide and fatty acid synthesis by engineering of the yeast acetyl-CoA carboxylase. J. Biotechnol. 187, 56 .

(30) Woods, A., Munday, M. R., Scott, J., Yang, X., Carlson, M., and Carling, D. (1994) Yeast SNF1 is functionally related to mammalian AMP-activated protein kinase and regulates acetyl-CoA carboxylase in vivo. J. Biol. Chem. 269, 19509-19515.
(31) Shi, S., Chen, Y., Siewers, V., and Nielsen, J. (2014) Improving production of malonyl coenzyme A-derived metabolites by abolishing Snf1-dependent regulation of Acc1. mBio 5, e01130-14.

(32) Besada-Lombana, P. B., Fernandez-Moya, R., Fenster, J., and Da Silva, N. A. (2017) Engineering Saccharomyces cerevisiae fatty acid composition for increased tolerance to octanoic acid. Biotechnol. Bioeng. 114, 1531-1538.

(33) Zhu, Z., Zhou, Y. J., Krivoruchko, A., Grininger, M., Zhao, Z. K., and Nielsen, J. (2017) Expanding the product portfolio of fungal type I fatty acid synthases. Nat. Chem. Biol. 13, 360-362.

(34) Generoso, W. C., Gottardi, M., Oreb, M., and Boles, E. (2016) Simplified CRISPR-Cas genome editing for Saccharomyces cerevisiae. J. Microbiol. Methods 127, 203-205.

(35) Gietz, R. D., and Schiestl, R. H. (2007) High-efficiency yeast transformation using the LiAc/SS carrier DNA/PEG method. Nat. Protoc. 2, 31-34.

(36) Bruder, S., Reifenrath, M., Thomik, T., Boles, E., and Herzog, K. (2016) Parallelised online biomass monitoring in shake flasks enables efficient strain and carbon source dependent growth characterisation of Saccharomyces cerevisiae. Microb. Cell Fact. 15, 115 .

(37) Sambrook, J., Fritsch, E., and Maniatis, T. (1989) Molecular Cloning: A Laboratory Manual, 2nd ed., Cold Spring Harbor Laboratory Press, Cold Spring Harbor, NY. 


\section{SUPPORTING INFORMATION}

Table S1. Yeast strains and plasmids used in this study.

\begin{tabular}{|c|c|c|}
\hline Strain & Characteristics & Reference \\
\hline CEN.PK113-7D & MATa; MAL2-8c; SUC2 & $\begin{array}{l}\text { Euroscarf, Frankfurt am Main, } \\
\text { Germany }\end{array}$ \\
\hline CEN.PK113-11C & MATa; MAL2-8c; SUC2; ura3-52; his3 $\Delta 1$ & $\begin{array}{l}\text { Euroscarf, Frankfurt am Main, } \\
\text { Germany }\end{array}$ \\
\hline LBY27 & CEN.PK113-11C $\triangle p y k 2:: p P D R 12-G F P$ & This study \\
\hline $\mathrm{RPY}^{21 / \mathrm{FAS}^{\text {R1834K }}}$ & 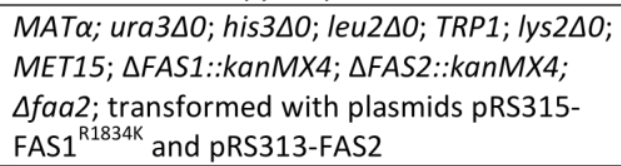 & 1 \\
\hline Plasmid & Characteristics & Reference \\
\hline \multicolumn{3}{|c|}{ Plasmids used for fermentations } \\
\hline p426pMET25-GFP & $2 \mu, U R A 3, A m p^{r}, p M E T 25-G F P-t C Y C 1$ & This study \\
\hline p426pPDR12-GFP & $2 \mu, U R A 3, A m p^{r}, p P D R 12-G F P-t C Y C 1$ & This study \\
\hline $\mathrm{pRS} 42 \mathrm{H}$ & $\begin{array}{l}2 \mu, h p h N T 1, A m p^{r}, \text { multiple cloning site } \\
\text { including EcoRV }\end{array}$ & 2 \\
\hline LBV17 & $\begin{array}{l}\text { pRS42H with pPGK1-TPO1-tTPO1 integrated } \\
\text { in ECORV site }\end{array}$ & This study \\
\hline LBV20 & $\begin{array}{l}\text { pRS42H with pPYK1-ACC1 }{ }^{\text {S659AS1157A }} \text {-tACC1 } \\
\text { integrated in EcoRV site }\end{array}$ & This study \\
\hline pRS315-FAS1 ${ }^{R 1834 K}$ & $\begin{array}{l}\text { CEN6/ARS4, LEU2, Amp }{ }^{r}, p A D H 2-F A S 1^{R 1834 K}{ }_{-} \\
\text {tFAS1 }\end{array}$ & 3 \\
\hline pRS313-FAS2 & CEN6/ARS4, HIS3, Amp ${ }^{r}, p A D H 2-F A S 2-t F A S 2$ & 3 \\
\hline \multicolumn{3}{|c|}{ Plasmids used for CRISPR/Cas9 } \\
\hline pRCC-K & $\begin{array}{l}2 \mu, k_{a n M X} A m p^{r}, p R O X 3-C a s 9^{o p t}-t C Y C 1, \\
\text { pSNR52-gRNA }\end{array}$ & 4 \\
\hline pRCC-K-PYK2 & pRCC-K with $g R N A$ for $P Y K 2$ locus & This study \\
\hline
\end{tabular}


Table S2. Oligonucleotides used in this study.

\begin{tabular}{|c|c|c|}
\hline Primer & Sequence 5'-3' & Application \\
\hline \multicolumn{3}{|c|}{ Plasmid construction or sequencing } \\
\hline LBP63 & $\begin{array}{l}\text { CAATTAACCCTCACTAAAGGGAACAAAAGCTGGAGCTGATATCTT } \\
\text { TGTTTTGCATTTTAC }\end{array}$ & \multirow{2}{*}{$\begin{array}{l}\text { Amplification of pPDR12 from } \\
\text { CEN.PK113-11C with } \\
\text { overhangs to the } \\
\text { p426pMET25-GFP backbone }\end{array}$} \\
\hline LBP64 & $\begin{array}{l}\text { CTACACCTGTAAACAATTCCTCGCCTTTAGACATTTTTTTATTAATAA } \\
\text { AGAACAATAAC }\end{array}$ & \\
\hline LBP103 & CTAATGTAGGCCATGGAAC & Sequencing of GFP \\
\hline LBP76 & $\begin{array}{l}\text { GGTCGACGGTATCGATAAGCTTGATCCCGGGATAGTAGAAAAAA } \\
\text { AAGGGGATATCACTAC }\end{array}$ & \multirow{2}{*}{$\begin{array}{l}\text { Amplification of TPO1-tTPO1 } \\
\text { from CEN.PK113-11C with } \\
\text { overhangs to } \mathrm{pRS} 42 \mathrm{H} \text { and } \\
\text { pPGK1, respectively }\end{array}$} \\
\hline LBP77 & $\begin{array}{l}\text { GTAATTATCTACTTTTTACAACAAATATAAAACAATGTCGGATCAT } \\
\text { TCTCCCATTTCTAA }\end{array}$ & \\
\hline LBP78 & $\begin{array}{l}\text { TTAGAAATGGGAGAATGATCCGACATTGTTTTATATTTGTTGTAA } \\
\text { AAAGTAGATAATTAC }\end{array}$ & \multirow{2}{*}{$\begin{array}{l}\text { Amplification of } p P G K 1 \text { from } \\
\text { CEN.PK113-11C with } \\
\text { overhangs to TPO1 and } \\
\text { pRS42H, respectively }\end{array}$} \\
\hline LBP79 & $\begin{array}{l}\text { GGTGGCGGCCGCTCTAGAACTAGTGGATCCCCCGGGAATTACCG } \\
\text { TCGCTCGTGATTTG }\end{array}$ & \\
\hline LBP80 & GCTACTGCTGAGAACCTG & \multirow[t]{2}{*}{ Sequencing of LBV17 } \\
\hline VSP159 & CGTGTGACAACAACAGCC & \\
\hline LBP81 & $\begin{array}{l}\text { GACTCACTATAGGGCGAATTGGGTACCGGGCCCCGACAGATTGG } \\
\text { GAGATTTTCATAGTAG }\end{array}$ & \multirow{2}{*}{$\begin{array}{l}\text { Amplification of } p P Y K 1 \text { from } \\
\text { CEN.PK113-11C with } \\
\text { overhangs to pRS42H and } \\
A C C 1 \text {, respectively }\end{array}$} \\
\hline LBP82 & $\begin{array}{l}\text { GAAGACTCGAATAAGCTTTCTTCGCTCATTGTGATGATGTTTTATT } \\
\text { TGTTTTGATTGGTG }\end{array}$ & \\
\hline LBP83 & $\begin{array}{l}\text { CACCAATCAAAACAAATAAAACATCATCACAATGAGCGAAGAAA } \\
\text { GCTTATTCGAGTCTTC }\end{array}$ & \multirow{6}{*}{$\begin{array}{l}\text { Amplification of } A C C 1 \text { with } \\
\text { S659A and S1157A and } t A C C 1 \\
\text { with overhangs to } p P Y K 1 \text { and } \\
\text { pRS42H, respectively }\end{array}$} \\
\hline RPP108 & CTATGGCAATCAAAAGACCACCATCAGCTAGTTGAC & \\
\hline RPP107 & GATATCATACTGCGTCAACTAGCTGATGG & \\
\hline RPP088 & CATATGACAAATCTGAAACAGCAACAGCCCTGTTCATAC & \\
\hline RPP087 & $\begin{array}{l}\text { ATGGGTATGAACAGGGCTGTTGCTGTTTCAGATTTGTCATATGTT } \\
\text { G }\end{array}$ & \\
\hline LBP84 & $\begin{array}{l}\text { GTACTCTGAAGGATCTGTTTGAGCGCTTCCATCGGGCCCATCGAA } \\
\text { TTCCTGCAGCCCGGG }\end{array}$ & \\
\hline LBP98 & CTTGTCATCCAATCTGTTC & \multirow[t]{8}{*}{ Sequencing of LBV20 } \\
\hline LBP99 & CCAAATAAGCACCGATACC & \\
\hline LBP100 & GCAACCATTCCTTAACAGG & \\
\hline LBP101 & GACATACAGAACTTCCAGG & \\
\hline LBP102 & GGAACATAGTTTGCAGTAGG & \\
\hline RPP89 & TTCGAAACCTTCTGTAGAAGCAACACAAAC & \\
\hline RPP90 & CGGTCAAGGAAAGAACTGAACAAATTGAAC & \\
\hline RPP109 & TCCAACTCTTGCCGTCATTTGC & \\
\hline RPP056 & CACACAGGAAACAGCTATGAC & \multirow{2}{*}{$\begin{array}{l}\text { Sequencing of p426pPDR12- } \\
\text { GFP, LBV17 and LBV20 }\end{array}$} \\
\hline LBP85 & CGTTACCCAACTTAATCGCC & \\
\hline \multicolumn{3}{|c|}{ Insertion of $p P D R 12-G F P$ in $P Y K 2$} \\
\hline LBP108 & $\begin{array}{l}\text { GTCCATTGTAAGATTACAACAAAAGCACTATCGGGCGAATTGGG } \\
\text { TACCGG }\end{array}$ & \multirow{2}{*}{$\begin{array}{l}\text { Amplification of } p P D R 12-G F P- \\
t C Y C 1 \text { from } p 426 p P D R 12-G F P \\
\text { with overhangs to } P Y K 2\end{array}$} \\
\hline LBP109 & $\begin{array}{l}\text { ATTAAGTAAAAAAAATAAGGACTTTAATTTTTTAGATATCTTTGTTT } \\
\text { TGCATTTTACATTC }\end{array}$ & \\
\hline LBP118 & CAGAGCGGTGAAACGCAAC & \multirow{2}{*}{$\begin{array}{l}\text { Amplification and sequencing } \\
\text { of } \triangle p y k 2:: p P D R 12-G F P-t C Y C 1\end{array}$} \\
\hline LBP119 & CGCAGTTTGCGAACATTACCTG & \\
\hline \multicolumn{3}{|c|}{ Amplification of CRISPR/Cas9 plasmid pRCC-K } \\
\hline WGP234 & $\begin{array}{l}\text { CTTGGTGGTGTTCGTCGTATCTCTTAATCATAGAAGCAGACAATG } \\
\text { GAG }\end{array}$ & \multirow[t]{3}{*}{$\begin{array}{l}\text { Amplification of pRCC-K with } \\
\text { gRNA for } P Y K 2\end{array}$} \\
\hline WGP235 & TGTTGTCTGACATTTTGAGAGTTAACACCGAAATTACCAAGGCTC & \\
\hline MGP193 & $\begin{array}{l}\text { TTGCAATTCGGAGTCCGCAAGTTTTAGAGCTAGAAATAGCAAGTT } \\
\text { AAAATAAGG }\end{array}$ & \\
\hline
\end{tabular}




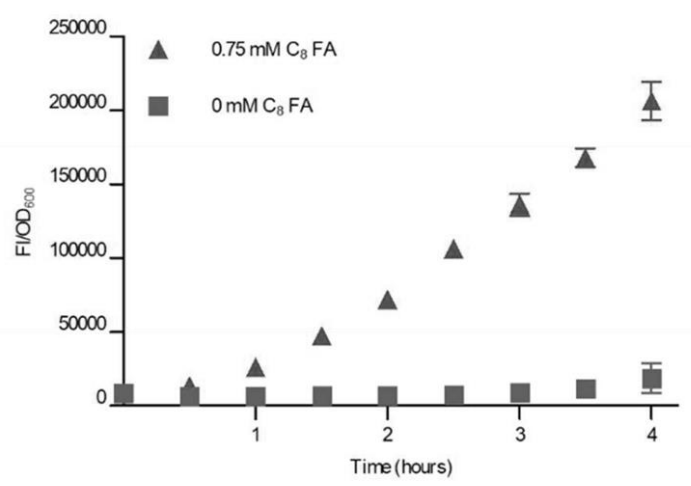

Figure S1. Time-dependent response of the biosensor in SCD medium with and without $\mathrm{C}_{8}$ fatty acids. Relative fluorescence intensity (fluorescence intensity $(\mathrm{FI})$ divided by $\mathrm{OD}_{600}$ ) of the plasmid-based sensor in response to 0 (grey squares) and $0.75 \mathrm{mM}$ (red triangles) $C_{8}$ fatty acids (FA), respectively. The background fluorescence of the biosensor strain not exposed to $C_{8} F A$ is very low over the entire time course. Error bars represent two technical replicates. 
A
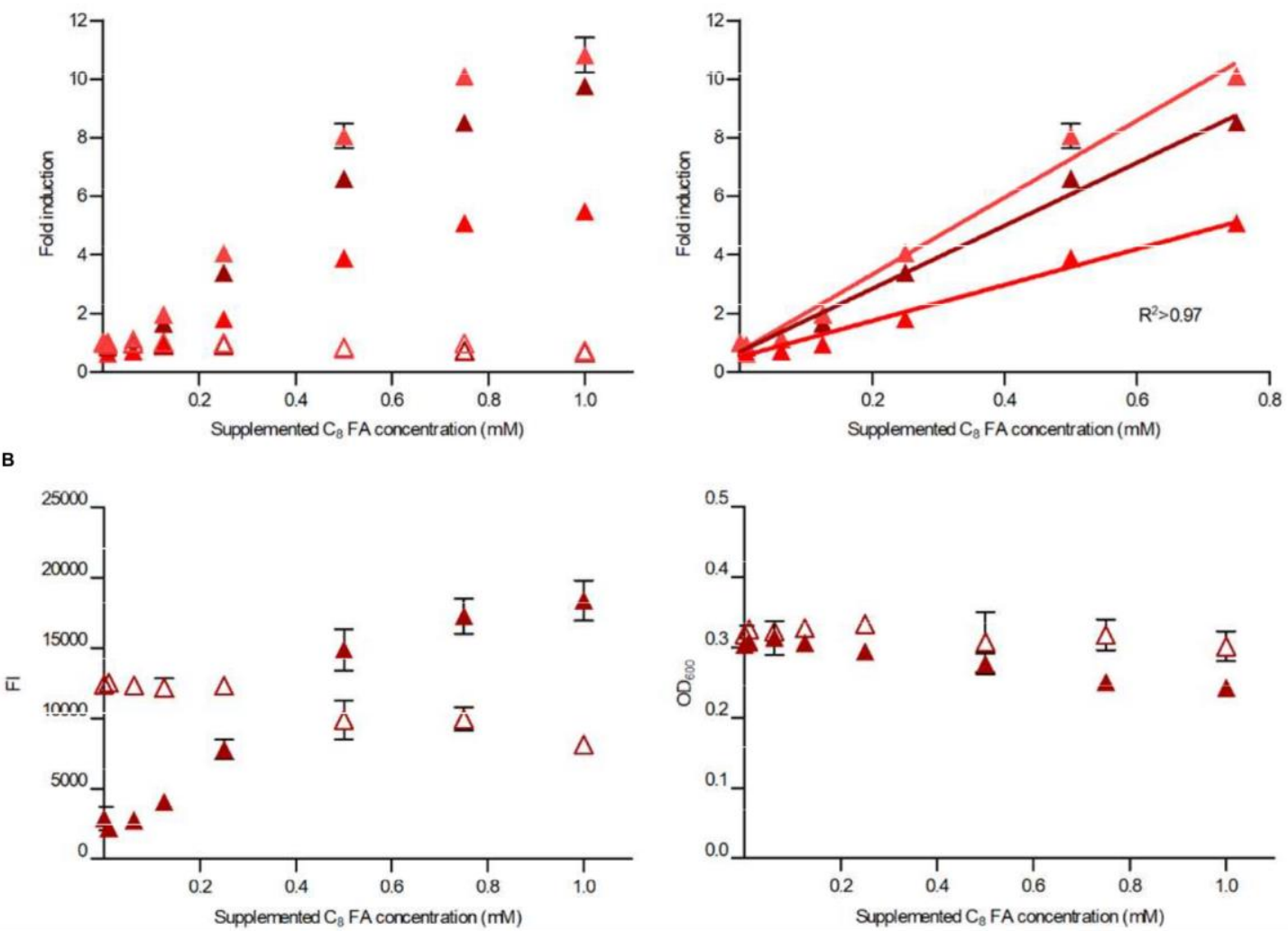

Figure S2. $C_{8}$ fatty acid-dependent response of the biosensor in SCD medium. (A) Response (left) and linear range (right) of the biosensor after 2 hours incubation with supplemented $C_{8}$ fatty acids (FA) in SCD medium. Shown are three biological replicates with two technical replicates each. For fold induction values, fluorescence intensities $(\mathrm{FI})$ were divided by optical densities $\left(\mathrm{OD}_{600}\right)$ and normalized to $\mathrm{FI} / \mathrm{OD}_{600}$ values of samples without $\mathrm{C}_{8}$ FA. (B) $\mathrm{FI}$ (left) and $\mathrm{OD}_{600}$ (right) of all three biological replicates. Filled triangles: CEN.PK113-11C + p426pPDR12-GFP. Clear triangles: CEN.PK113-11C + p426pMET25-GFP. 

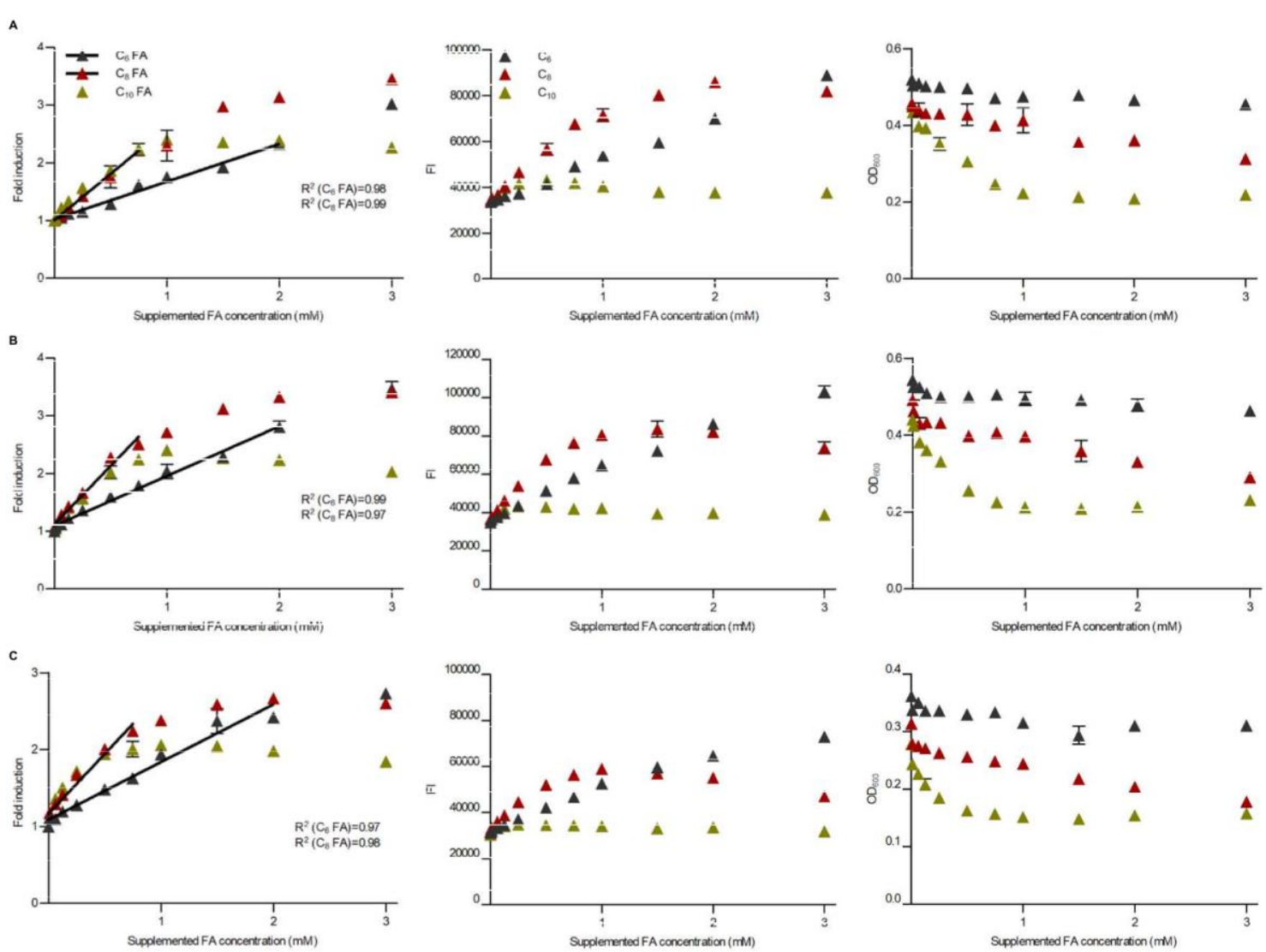

Figure S3. $C_{6}, C_{8}$ and $C_{10}$ fatty acid-dependent response of the biosensor in YPD medium of three biological replicates $(A, B, C)$. Response and linear range (left), fluorescence intensities ( $F l$; middle) and optical densities $\left(\mathrm{OD}_{600}\right.$; right) after 4 hours incubation with supplemented $C_{6}, C_{8}$ or $C_{10}$ fatty acids (FA) of all three biological replicates. Linear ranges were only observed in response to $C_{6}$ and $C_{8} F A s$ (left). Error bars represent two technical replicates. For fold induction values, Fls were divided by $\mathrm{OD}_{600}$ values and normalized to $\mathrm{Fl} / \mathrm{OD}_{600}$ values of samples without FA. 

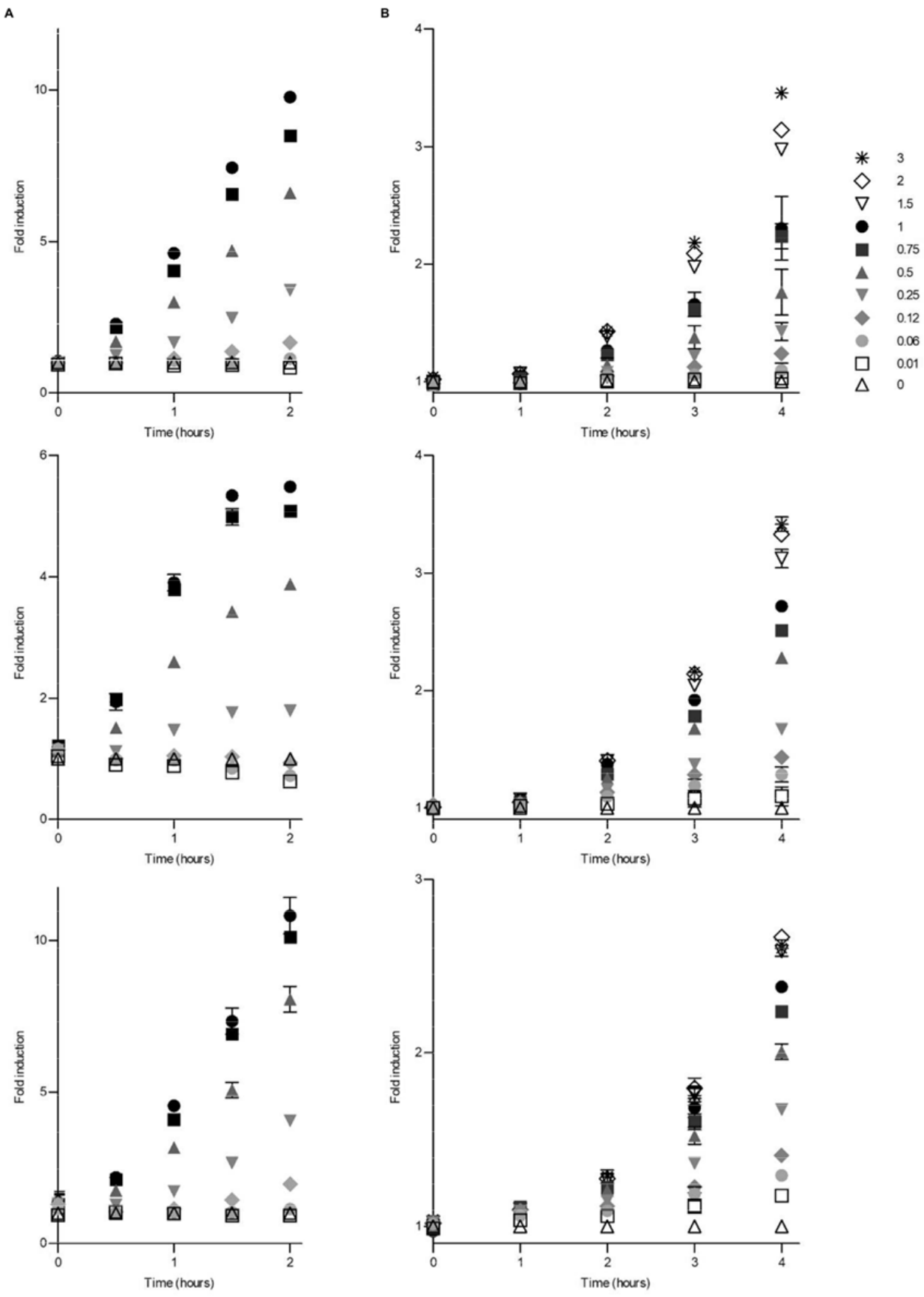

Figure S4. Time-dependent response of the biosensor to different $\mathrm{C}_{8}$ fatty acid concentrations. Response over a 2 hour incubation period to supplemented 0-1 mM $\mathrm{C}_{8}$ fatty acids (FA) in SCD (A) and over a 4 hour incubation period to supplemented 0-3 $\mathrm{mM} \mathrm{C}_{8}$ FA in YPD medium (B) of three biological replicates. Error bars represent two technical replicates. For fold induction values, fluorescence intensities (FI) were divided by optical densities $\left(\mathrm{OD}_{600}\right)$ and normalized to $\mathrm{FI} / \mathrm{OD}_{600}$ values of samples without $\mathrm{C}_{8} \mathrm{FA}(0 \mathrm{mM})$. 

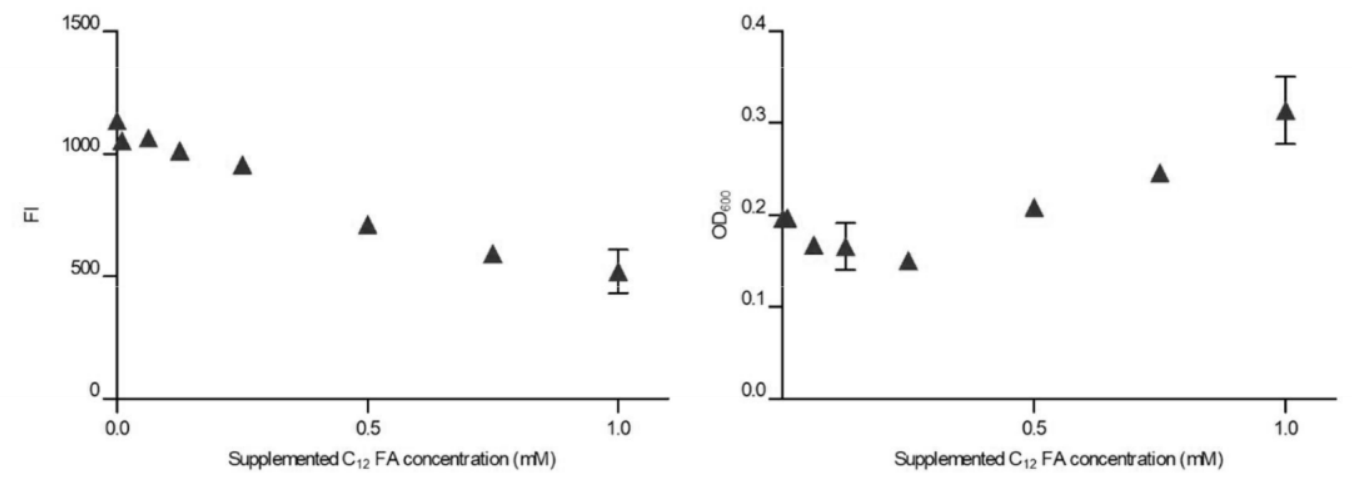

Figure S5. $\mathrm{C}_{12}$ fatty acid-dependent growth and fluorescence of the biosensor. Fluorescence intensities (FI; left) and optical densities $\left(\mathrm{OD}_{600}\right.$; right) in response to supplementation with $\mathrm{C}_{12}$ fatty acids (FA) after 4 hours incubation in YPD medium. Error bars represent two technical replicates. 

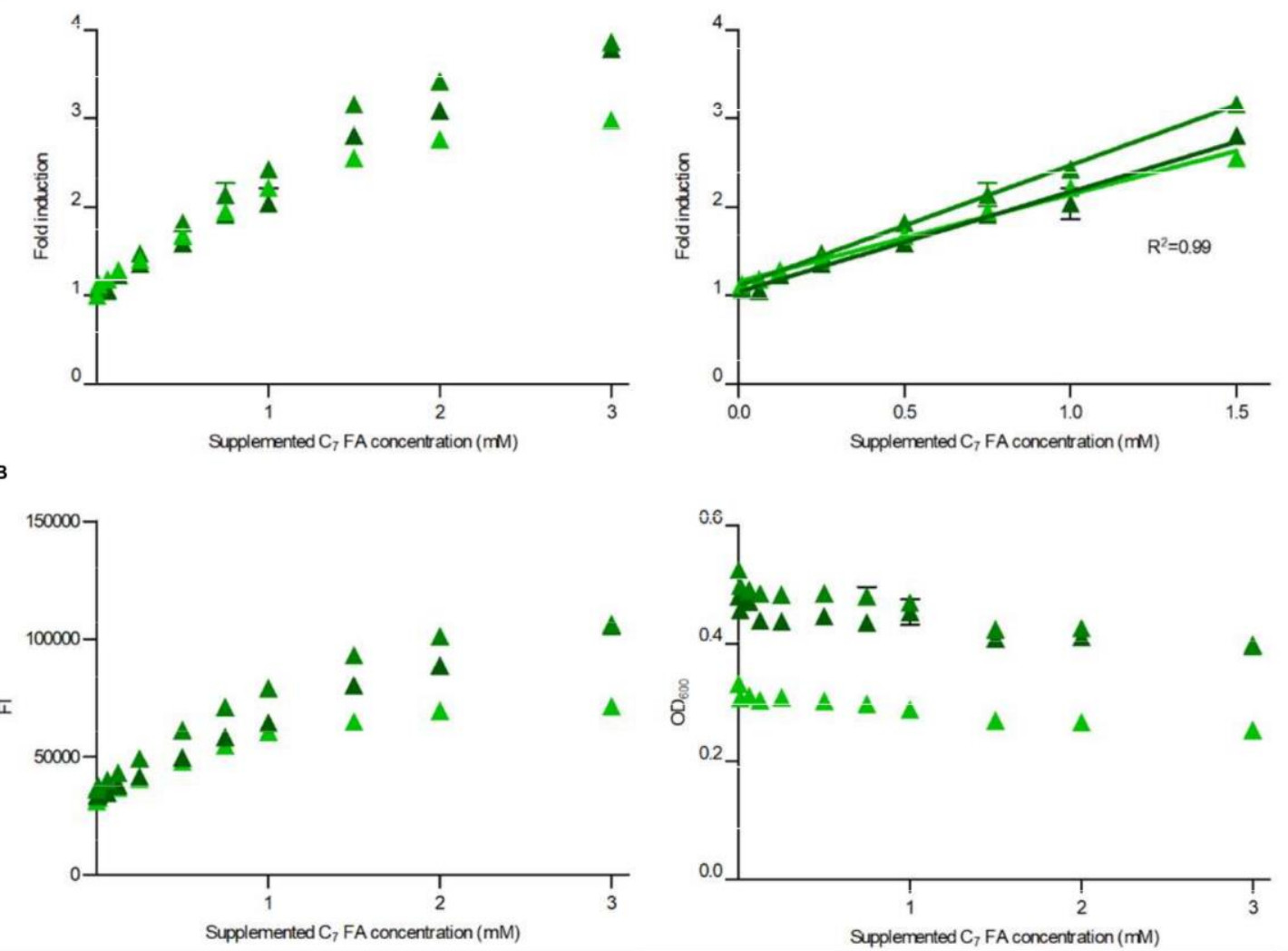

Figure S6. $C_{7}$ fatty acid-dependent response of the biosensor. (A) Response (left) and linear range (right) of the biosensor after 4 hours incubation with supplemented $C_{7}$ fatty acids (FA) in YPD medium. For fold induction values, fluorescence intensities $(\mathrm{FI})$ were divided by optical densities $\left(\mathrm{OD}_{600}\right)$ and normalized to $\mathrm{Fl} / \mathrm{OD}_{600}$ values of samples without $\mathrm{C}_{7} \mathrm{FA}$. (B) $\mathrm{FI}$ (left) and $\mathrm{OD}_{600}$ (right) values of all three biological replicates. Shown are three biological replicates with error bars representing two technical replicates. 
A

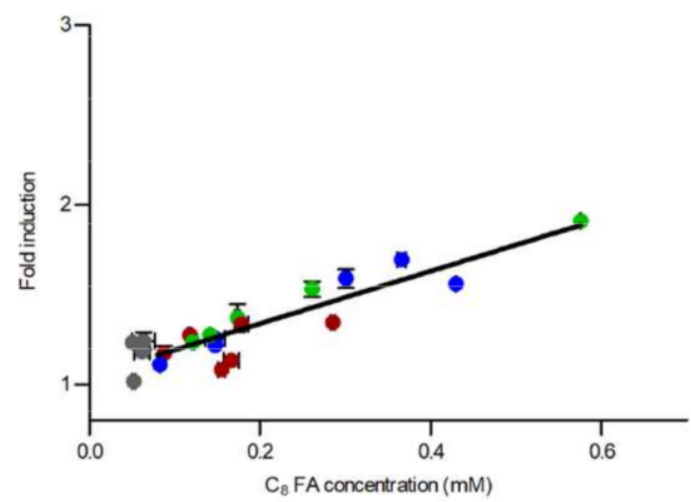

B

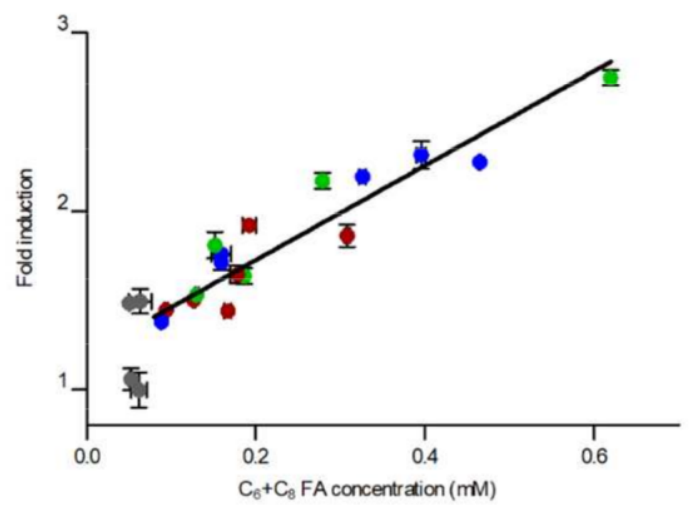

Figure S7. Biosensor response to fatty acids in S. cerevisiae culture supernatants and correlation to GC measurement. (A) Linear correlation of the fold induction of biosensor signal in 0.25 dilutions of culture supernatants with $\mathrm{GC}$ measurements of $\mathrm{C}_{8}$ fatty acids (FA) of the same supernatants. (B) Linear correlation of the fold induction of biosensor signal in 0.5 dilutions of culture supernatants with $G C$ measurements of $C_{6}$ and $\mathrm{C}_{8}$ FA of the same supernatants. Strains: CEN.PK113-7D (grey), RPY21/FAS ${ }^{\text {R1834K } / p R S 42 H ~(r e d), ~ R P Y 21 / F A S ~}{ }^{\text {R1834K } / ~}$

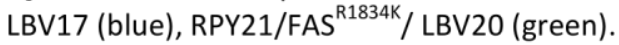

\section{REFERENCES}

(1) Henritzi, S., Fischer, M., Grininger, M., Oreb, M., and Boles, E. (2018) An engineered fatty acid synthase combined with a carboxylic acid reductase enables de novo production of 1-octanol in Saccharomyces cerevisiae. Biotechnol. Biofuels 11:150.

(2) Taxis, C., and Knop, M. (2006) System of centromeric, episomal, and integrative vectors based on drug resistance markers for Saccharomyces cerevisiae. Biotechniques 40, 73-78.

(3) Gajewski, J., Pavlovic, R., Fischer, M., Boles, E., and Grininger, M. (2017) Engineering fungal de novo fatty acid synthesis for short chain fatty acid production. Nat. Commun. 8, 14650.

(4) Generoso, W. C., Gottardi, M., Oreb, M., and Boles, E. (2016) Simplified CRISPR-Cas genome editing for Saccharomyces cerevisiae. J. Microbiol. Methods 127, 203-205. 


\subsection{Engineering Saccharomyces cerevisiae for production of fatty acids and their derivatives}

Declaration of author contributions to the publication:

Engineering Saccharomyces cerevisiae for production of fatty acids and their derivatives [review]

Status: published, December 2020

Book: The Mycota - Genetics and Biotechnology $3^{\text {rd }}$ edition, Springer

Type of publication: book chapter

Contributing authors: Leonie Baumann (LB), Florian Wernig (FW), Sandra Born (SB) \& Mislav Oreb (MO)

Contributions of doctoral candidate and co-authors

(1) Concept and design

Doctoral candidate LB: $35 \%$

Co-authors FW, SB, MO: 15\%, 15\%, 35\%

(2) Conducting tests and experiments

$\mathrm{n} / \mathrm{a}$

(3) Literature collection

Doctoral candidate LB: $35 \%$

Co-authors FW, SB, MO: 15\%, 15\%, 35\%

(4) Analysis and interpretation of data

Doctoral candidate LB: $35 \%$

Co-authors FW, SB, MO: 15\%, 15\%, 35\%

(5) Drafting of manuscript

Doctoral candidate LB: $35 \%$

Co-authors FW, SB, MO: 15\%, 15\%, 35\% 


\section{Engineering Saccharomyces cerevisiae for Production of Fatty Acids and Their Derivatives}

Leonie Baumann ${ }^{1}$, Florian Wernig ${ }^{1}$, Sandra Born $^{1}$, Mislav Oreb

\section{CONTENTS}

I. Introduction .........................339

II. Providing Carbon, Redox Power, and Energy for Fatty Acid Synthesis: The Options for Precursor Supply Routes ................340

A. Engineering the Pyruvate Dehydrogenase Bypass ............................341

B. ATP-Independent Pyruvate-to-Acetyl-CoA Routes ..............................343

C. Phosphoketolase Pathway ...............344

D. Citrate-Oxaloacetate Shuttle .............345

E. Strategies to Manipulate NADPH Level Independently of Precursor Supply Routes346

F. Engineering Malonyl-CoA Supply ........346

III. Strategies for Chain Length Control ..........348

A. Manipulation of the Natural Fatty Acid Biosynthesis and Elongation Machinery .. 348

B. Reversal of $\beta$-Oxidation as an Orthogonal Pathway for Fatty Acid Biosynthesis ...... 349

IV. Chassis Engineering ......................351

A. Elimination of By-product Formation and Fatty Acid Degradation ................351

B. Engineering Yeast Product Tolerance and Excretion ........................... 352

C. Dynamic Pathway Control and Biosensors 354

V. S. cerevisiae as a Production Platform for

Fatty Acid Derivatives ...................356

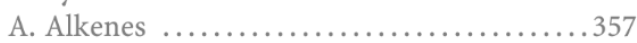

B. Dicarboxylic Acids ..................357

C. Fatty Aldehydes, Alkanes, and Alcohols ...357

D. Wax Esters and Fatty Acid Ethyl Esters ...358

VI. Optimization of Fermentation Conditions

VII. Conclusions .

References

\footnotetext{
${ }^{1}$ Faculty of Biological Sciences, Institute of Molecular Biosciences, Goethe-University Frankfurt, Frankfurt, Germany; e-mail: M.Oreb@bio.uni-frankfurt.de
}

\author{
Abbreviations \\ AcCoA Acetyl-CoA \\ AcP Acetyl-phosphate \\ ALE Adaptive laboratory evolution \\ CoA Coenzyme A \\ FA Fatty acid(s) \\ FACS Fluorescence-activated cell sorting \\ FAEE Fatty acid ethyl ester(s) \\ GAP Glyceraldehyde-3-phosphate \\ gTME Global transcription machinery engi- \\ neering \\ MalCoA Malonyl-CoA \\ PPP Pentose phosphate pathway \\ SE Steryl ester \\ TAG Triacylglycerol \\ TCA Tricarboxylic acid \\ TE Thioesterase \\ X5P Xylulose 5-phosphate
}

\section{Introduction}

Fatty acids (FAs) and their derivatives, such as fatty alcohols, dicarboxylic acids, FA esters, alkanes, and alkenes, are frequently summarized under the generic term "oleochemicals." The diversity of physicochemical properties of oleochemicals is determined by their functional groups and by the length of the aliphatic chains. This is reflected by a variety of products containing these classes of compounds, including fuels, lubricants, surfactants, detergents, cosmetics, food additives, and pharmaceuticals. Consequently, the production volume of different oleochemicals is measured in millions of 
tons per year (Rupilius and Ahma 2006), and the demand is steadily increasing. They are currently produced from petroleum (in which case the term "petrochemicals" is also used) or biomass-derived (mainly plant) fats. While the concerns associated with the exploitation of fossil resources have been known for a long time, there is an increasing awareness of problems caused by mass cultivation of oily plants (e.g., palms), for instance, excessive land use and water consumption (Schmidt 2015). The biorefinery concept, in which engineered microbes are used to convert various biomass feedstocks into desired products (Nielsen et al. 2013), offers a potentially more effective exploitation of resources and thereby reduces the environmental impact compared to extraction from plants. Indeed, significant advances in production of oleochemicals with different chain lengths and functionalities have been achieved in diverse microbial hosts (for review, see Yu et al. 2014; d'Espaux et al. 2015; Pfleger et al. 2015; Sarria et al. 2017; Marella et al. 2018; Xue et al. 2018; Zhang et al. 2018). Since rapid progress is made in the field, we feel that a critical re-evaluation of the literature is justified. Here, we will focus on strategies developed in S. cerevisiae, one of the best characterized and most popular biotechnological host organisms (see also Chap. 13 by Schmelzer et al.), in which highest yields of microbially produced FA have been recently reported ( $\mathrm{Yu}$ et al. 2018). Different approaches for engineering precursor and cofactor supply, chain length control, elimination of by-product formation, and overcoming product toxicity as well as the development of biosensors for accelerating the screening for best performing strains are summarized and evaluated. Throughout the manuscript, we consciously do not collect all data on achieved titers and yields for different FAderived products, as another recent review has provided a very detailed summary (FernandezMoya and Da Silva 2017). Strategies that have been developed in other host organisms (e.g., Escherichia coli) are occasionally discussed, if they appear suitable to expand the pathways already established in S. cerevisiae. Lastly, we give an overview of the production of FAderived products in $S$. cerevisiae as well as methods for optimizing fermentation condi- tions, enabling the scale-up of current labscale fermentation processes.

\section{Providing Carbon, Redox Power, and Energy for Fatty Acid Synthesis: The Options for Precursor Supply Routes}

S. cerevisiae, an ethanologenic yeast, only requires relatively small amounts of $\mathrm{FA}$ as building blocks of membrane lipids. The FA synthesis naturally occurs in the cytosol by the FA synthase (FAS) that uses acetyl-CoA (AcCoA) and its derivative malonyl-CoA (MalCoA) as precursor molecules. AcCoA metabolism in yeast is compartmentalized, whereby the major part is synthesized inside mitochondria via the pyruvate dehydrogenase (PDH) complex (Krivoruchko et al. 2015) and the mitochondrial AcCoA cannot be exported to the cytosol (van Rossum et al. 2016a, c). The native pathway for the synthesis of cytosolic AcCoA in S. cerevisiae is referred to as pyruvate dehydrogenase (PDH) bypass, and it diverts only a minor part of the pyruvate produced from glucose (Pronk et al. 1996). Following decarboxylation of pyruvate by pyruvate decarboxylases, acetaldehyde is oxidized to acetate by acetaldehyde dehydrogenases (ALDs), and acetate is subsequently ligated to $\mathrm{CoA}$ by the acetyl-CoA synthetases (ACS). This reaction is thermodynamically driven by hydrolysis of ATP to AMP and inorganic pyrophosphate, which is subsequently hydrolyzed by the inorganic pyrophosphatase. Thus, each AcCoA molecule is synthesized at the expense of two ATP equivalents by the PDH bypass. This reaction scheme implies that the glycolytic ATP supply (net two ATP moles per mol glucose) is insufficient, because energy equivalents are also needed for cell proliferation and maintenance. Thus, a significant proportion of the available (sugar) substrate must be diverted to the tricarboxylic acid (TCA) cycle and respiration for the supply of energy equivalents. This reduces the attainable product yield (van Rossum et al. 2016b) and, considering that most FA and derived products have a relatively low commercial value, makes their production through the PDH bypass uneconomical. 
AcCoA is not only a building block of FA but also of many compound classes that are of interest in biotechnology, such as isoprenoids (e.g., artemisinic acid; Paddon et al. 2013), polyesters (e.g., polyhydroxybutyric acid; Kocharin et al. 2012), polyketides (e.g., 6methylsalicylic acid; Wattanachaisaereekul et al. 2008), or flavonoids (e.g., naringenin; Koopman et al. 2012). To avoid constraints such as limited metabolic capacity of organelles, availability of cofactors, and transport of products across organellar membranes, heterologous pathways for production of these compounds are preferably expressed in the cytosol. Therefore, significant effort has been devoted to improving the cytosolic AcCoA supply in S. cerevisiae. An excellent review article (van Rossum et al. 2016b) provided a systematic analysis of possible pathways to optimize the supply of cytosolic AcCoA under consideration of reaction stoichiometry, energy conservation, and maximally attainable yields for four model AcCoA-derived compounds including FA. Here, we will revisit the current literature that exploited some of those different possibilities (summarized in Fig. 1) to produce FA and other AcCoA-derived products. Since the provision of reducing equivalents-either NADH or NADPH-is equally important for FA production and intrinsically dependent on the choice of the AcCoA synthesis route, the redox cofactor supply will be discussed throughout this chapter. The synthesis of FA can be engineered to occur either via FAS that strictly requires NADPH or by reversing $\beta$-oxidation, a FA degradation pathway, which allows for more flexibility regarding the cofactor dependence (see Sect. III.B). Therefore, the choice of the appropriate AcCoA pathway also depends on the synthesis mode of FA.

\section{A. Engineering the Pyruvate Dehydrogenase Bypass}

In most proof-of-concept studies, the production of AcCoA-derived products in S. cerevisiae relied on the native PDH bypass or engineered variants of it, without introducing heterologous AcCoA routes. Even when various alternative AcCoA pathways were introduced, the native
PDH bypass was present in the background of all engineered strains, with one exception (Meadows et al. 2016) known to us (see Sect. II.C).

An increased flux through the PDH bypass can be achieved by redirecting acetaldehyde away from reduction to ethanol by alcohol dehydrogenases (ADHs) toward oxidation to acetate by ALDs. In a first study (aiming to increase the production of the isoprenoid amorphadiene), it could be shown that overexpressing the acetaldehyde dehydrogenase ALD6 alone substantially increased the accumulation of acetate (Shiba et al. 2007). Since Ald6 is NADP-specific, this step is also important to provide NADPH required by FAS. Next, the authors tested different ACS variants, whereby only the expression of a heterologous enzyme from Salmonella enterica, mutated to prevent inactivation by acetylation $\left(\right.$ SeACS $^{\mathrm{L641P}}$ ), led to a substantial increase in amorphadiene production (Shiba et al. 2007). In the same study, the overexpression of endogenous enzymes had no (for Acs2) or little (for Acs1) effect on amorphadiene titers, which was attributed to negative regulatory mechanisms at the post-translational level, possibly acetylation. In subsequent studies, a positive effect of combined Ald6/SeACS ${ }^{\mathrm{L} 641 \mathrm{P}}$ overexpression on production of several AcCoA-derived products, including FA derivatives such as FA ethyl esters (FAEEs) and hexadecanol, was confirmed by different groups (Chen et al. 2013b; Krivoruchko et al. 2013; Jong et al. 2014; Lian et al. 2014; Feng et al. 2015).

As a strategy to utilize the major fermentation product ethanol for AcCoA synthesis, the $\mathrm{ADH}$ isoform II (Adh2), which is the main enzyme responsible for oxidation of ethanol to acetaldehyde, was co-expressed with Ald6/SeACS ${ }^{\mathrm{L} 641 \mathrm{P}}$ in some variants (Chen et al. 2013b; Krivoruchko et al. 2013; Jong et al. 2014; Feng et al. 2015). Unfortunately, the effect of Adh2 overexpression alone remained unclear, since it was overexpressed only in combination with other enzymes (e.g., acetoacetyl-CoA thiolase Erg10) that affect the product yield (Kocharin et al. 2012; Chen et al. 2013b; Krivoruchko et al. 2013) or together with Ald6 and SeACS ${ }^{\mathrm{L} 641 \mathrm{P}}$, without a direct comparison to a control not overexpressing Adh2 (Jong et al. 2014; Feng et al. 2015). Considering that the interconversion of acetaldehyde and ethanol is rather driven by the chemical equilibrium, it remains yet to be demonstrated that Adh2 overexpression indeed diverts the flux toward AcCoA in engineered strains in the presence of glucose. 


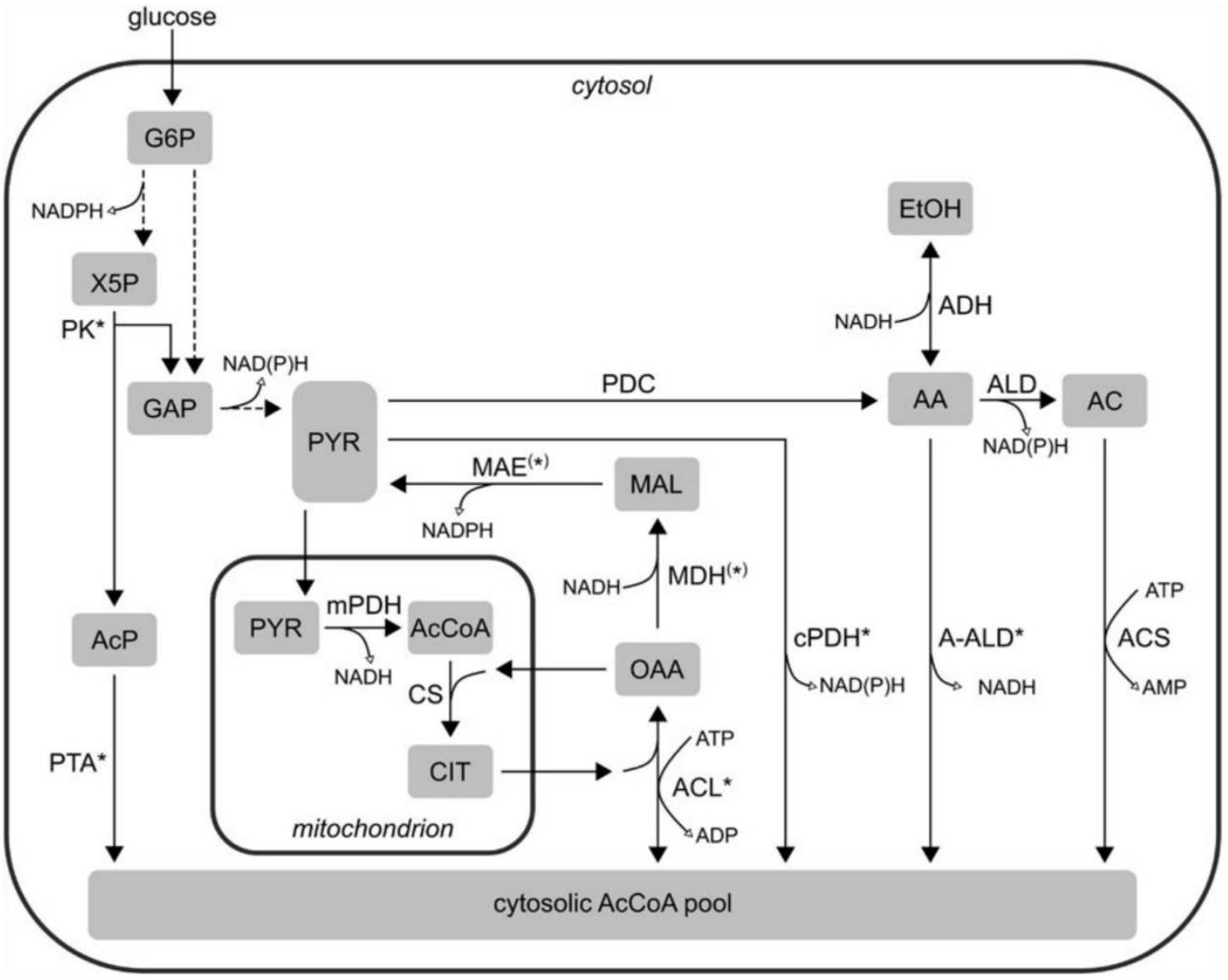

Fig. 1 A simplified scheme of precursor supply pathways for production of acetyl-CoA (AcCoA) derivatives in the cytosol of $S$. cerevisiae. Only the relevant pathway intermediates are shown in gray boxes: glucose-6-phosphate (G6P), xylulose 5-phosphate (X5P), glyceraldehyde-3-phosphate (GAP), pyruvate (PYR), acetaldehyde (AA), ethanol (EtOH), acetate (AC), acetyl-phosphate (AcP), citrate (CIT), oxaloacetate (OAA), and malate (MAL). Solid lines depict singlestep reactions, whereas dashed lines stand for multiple enzymatic steps. Reversibility of reactions is indicated by double-headed arrows only if relevant in the context of AcCoA supply. For reactions that can be catalyzed by enzymes with different specificities, the generic notation "NAD $(\mathrm{P}) \mathrm{H}$ " is used; otherwise, the specific cofactor is denoted. For clarity, only the reduced form of the

A logical approach to boost the production of cytosolic AcCoA via the PDH bypass is the elimination of ethanol production by deleting the ADH genes. Li et al. (2014) first demonstrated that the deletion of $A D H 1$ is beneficial redox cofactors is shown. The reaction stoichiometries are not considered, and energy equivalents are shown only for the AcCoA supply reactions. Enzyme names are abbreviated as follows: $P K$ phosphoketolase, PTA phosphotransacetylase, $P D C$ pyruvate decarboxylase, $A L D$ aldehyde dehydrogenase, $m P D H$ mitochondrial pyruvate dehydrogenase, $c P D H$ cytosolic pyruvate dehydrogenase, CS citrate synthase, ACL ATP citrate lyase, $M D H$ malate dehydrogenase, MAE malic enzyme, $A-A L D$ acetylating aldehyde dehydrogenase, ACS acetyl-CoA synthetase; heterologous enzymes are marked with an asterisk * and endogenous enzymes that are expressed in a different compartment with an asterisk in brackets $\left.{ }^{(}\right)$. Transporters are not shown. Influx/efflux of (pyro)phosphate, carbon dioxide, and coenzyme A ( $\mathrm{CoA})$ moieties is omitted for clarity

for FA production. Lian et al. (2014) went a step further and deleted the isoforms $A D H 1$ and $A D H 4$ with a concomitant deletion of glycerol3-phosphate dehydrogenases GPD1 and GPD2 to prevent accumulation of glycerol, a trait pre- 
viously observed in $\mathrm{ADH}$-deficient strains (Smidt et al. 2012). This elimination of competing products already increased the flux toward AcCoA, measured as an increase in n-butanol production through a simultaneously expressed heterologous pathway (Lian et al. 2014). Schadeweg and Boles (2016b) could later show that a deletion of $\mathrm{ADH}$ isoforms 15 , even without a GPD deletion, substantially increases the production of n-butanol through a similarly designed pathway. When Ald6/ SeACS $^{\text {L641P }}$ were overexpressed in an ADH-/ GPD-deficient strain, the production of $n$ butanol unexpectedly decreased due to a strong accumulation of acetate (Lian et al. 2014). Conversely, overexpression of SeACS ${ }^{\text {L641P }}$ alone improved the productivity in the same strain background (Lian et al. 2014; Lian and Zhao 2015). Taken together, the analyses performed in ADH positive (Shiba et al. 2007) and negative (Lian et al. 2014; Lian and Zhao 2015) strains suggest that the balance of ALD and ACS activity is a critical and context-dependent variable in optimizing the flux through the cytosolic $\mathrm{PDH}$ bypass due to the rate-limiting role of ACS and the accumulation of toxic acetate levels.

\section{B. ATP-Independent Pyruvate-to-Acetyl-CoA Routes}

The high energy requirement makes the $\mathrm{PDH}$ bypass a rather inefficient precursor supply pathway for manufacturing AcCoA-derived products. Therefore, several alternative ATPindependent $\mathrm{AcCoA}$ yielding pathways were tested in S. cerevisiae for different products. One possibility to convert acetaldehyde to AcCoA is via acetylating aldehyde dehydrogenases (A-ALD). Their functionality in yeast was demonstrated by the ability to complement the growth defect of an ACS-deficient strain (Kozak et al. 2014b) and to replace endogenous acetaldehyde dehydrogenases (Kozak et al. 2016). Schadeweg and Boles (2016b) demonstrated increased n-butanol production via reverse $\beta$-oxidation when they overexpressed A-ALD from $E$. coli, mutated to favor the (nonphysiological) reaction direction from acetalde- hyde to AcCoA (adhE ${ }^{\mathrm{A} 267 \mathrm{~T} / \mathrm{E} 568 \mathrm{~K}}$; MembrilloHernandez et al. 2000) in an ADH-deficient strain. However, a positive effect of A-ALD overexpression could only be seen when the supply of coenzyme A (CoA) was concomitantly increased by overexpression of a heterologous pantothenate kinase (coaA from E. coli) and pantothenate feeding, demonstrating that the availability of not only acetyl moieties but also of the coenzyme is a factor limiting the synthesis of AcCoA (Schadeweg and Boles 2016b).

This notion likely applies to all AcCoA pathways, as the overexpression of the pantothenate kinase and pantothenate supplementation was also beneficial in combination with an engineered PDH bypass (Ald6/ $\mathrm{SeACS}^{\mathrm{L} 641 \mathrm{P}}$ overexpression) in a strain constructed for production of the flavonoid naringenin (Liu et al. 2017). The endogenous pantothenate supply can be improved by overexpressing the polyamine oxidase Fms1 (Schadeweg and Boles 2016a), which catalyzes the limiting step of its biosynthesis.

A-ALD pathway is a very promising alternative to the $\mathrm{PDH}$ bypass to produce AcCoA derivatives, owing to its lower energy requirement. However, the theoretically attainable yield for FA produced by FAS is lower when A-ALD is used compared to PDH bypass (van Rossum et al. 2016b) due to cofactor incompatibility (the A-ALD pathway yields $\mathrm{NADH}$, while FAS requires NADPH). Thus, only if the FAs are produced via reverse $\beta$-oxidation, which can be engineered to utilize exclusively $\mathrm{NADH}$, the implementation of the A-ALD pathway appears feasible. The same is true for other alternative pathways that convert pyruvate to AcCoA in an ATP-independent manner and thereby yield $\mathrm{NADH}$, namely, (1) pyruvate formate lyase (PFL) combined with formate dehydrogenase, and (2) cytosolic pyruvate dehydrogenase complex (cPDH) (van Rossum et al. 2016b). Although the functionality of PFL in yeast cytosol has been demonstrated (Waks and Silver 2009; Kozak et al. 2014b), it has not been used for high-level production of FA or other AcCoA derivatives so far, due to its complex biochemical properties, including strong oxygen sensitivity (Knappe et al. 1969). In contrast, a significant improvement of n-butanol produc- 
tion was reported when a bacterial PDH or the native PDH components lacking the mitochondrial targeting sequence were overexpressed in yeast cytosol (Lian et al. 2014).

It has to be noted, however, that the cytosolic PDH activity was not directly demonstrated in this study. In another study (Kozak et al. 2014a), it has been shown that the activity of PDH in the yeast cytosol is strictly dependent on supplementation of lipoic acid, a cofactor that is normally synthesized inside mitochondria and must be covalently linked to the E2 subunit of $\mathrm{PDH}$. Moreover, overexpression of enzymes involved in lipoylation of E2 in the cytosol is crucial (Kozak et al. 2014a). Since Lian et al. (2014) did neither supplement lipoic acid nor express the components of the lipoylation machinery, the mechanism by which $\mathrm{CPDH}$ led to an improvement of n-butanol production in their work remains enigmatic.

Of particular interest for FA synthesis via the (NADPH-dependent) FAS would be an implementation of NADPH-yielding $\mathrm{CPDH}$ variants. Indeed, a mutated ( $\mathrm{NADP}^{+}$-accepting) PDH was developed (Bocanegra et al. 1993) and implemented to produce the polyketide triacetic acid lactone in yeast cytosol. Although the cytosolic PDH activity was not directly measured, this strategy led to a significantly increased NADPH/NADP ratio and AcCoA levels in whole cell extracts as well as to improved product titers in fermentations (Cardenas and Da Silva 2016). Since this approach has the same cofactor yield (one NADH and one NADPH per molecule AcCoA) but a better energetic balance compared to the PDH bypass, it could potentially improve the production of FA. However, the study did not address the abovementioned requirement for lipoylation, and this aspect will therefore require further investigation before this approach can be employed for FA production.

\section{Phosphoketolase Pathway}

Among all individually considered alternative AcCoA pathways, the phosphoketolase/transacetylase variant was predicted to enable the highest yield of FAS-derived FA ( $87 \%$ of the theoretical maximum), when the carbon flux is rerouted from glycolysis to the oxidative pentose phosphate pathway (PPP) to increase the NADPH pool (van Rossum et al. 2016b). In this scheme, glucose-6-phosphate is converted to xylulose 5-phosphate (X5P) through a consecutive action of the endogenous enzymes glucose-6-phosphate dehydrogenase, 6phosphogluconolactonase, 6-phosphoglu conate dehydrogenase, and D-ribulose-5-phosphate 3-epimerase, whereby the first and the third reaction yield one NADPH molecule each (for a review of the PPP, see Stincone et al. 2015). X5P is then converted to acetylphosphate (AcP) and glyceraldehyde-3-phosphate (GAP) by a heterologous phosphoketolase (PK). Subsequently, AcP is directly converted to AcCoA by a heterologous phosphotransacetylase (PTA). The PK/PTA pathway was expressed in a strain engineered to produce FAEE and indeed led to a significant increase of product titers compared to the reference strain containing only the native PDH bypass (Jong et al. 2014).

Another variant to convert PKA-derived AcP to AcCoA involves the acetate kinase (ACK), which yields acetate and ATP by transferring the phosphate residue from AcP to ADP. Although ATP is produced in this reaction, the production of $\mathrm{AcCoA}$ via this route is energetically less favorable than via PTA since two ATP have to be invested into the subsequent activation of acetate by ACS (see above). The implementation of this strategy led to a significant improvement of polyhydroxybutyrate (Kocharin et al. 2013) and, to a lower extent than with the PK/PTA variant, FAEE production (Jong et al. 2014)

In one recent study (Meadows et al. 2016), the PK/PTA pathway was combined with the AALD to produce the isoprenoid farnesene, as the combinatorial configuration was calculated to lead to the highest possible product yields (van Rossum et al. 2016b). Interestingly, the authors found that AcP produced by $\mathrm{PK}$ is partly dephosphorylated by endogenous (promiscuous) glycerol-3-phosphate phosphatases and their activity needs to be reduced to favor the PTA reaction. This study stands out as the only one in which the endogenous PDH bypass was fully substituted (by ald6 acs 1 acs 2 deletion) with heterologous AcCoA synthesis routes 
(A-ALD and PK/PTA). Indeed, this approach led to the highest yields ever reported of an isoprenoid produced in $S$. cerevisiae and impressively demonstrated how an "ideal" combination of precursor supply pathways based on stoichiometric analyses can be designed to produce AcCoA derivatives at an industrially competitive level.

\section{Citrate-Oxaloacetate Shuttle}

The major breakthroughs regarding production of oleochemicals in $S$. cerevisiae have been recently achieved by implementing the citrateoxaloacetate shuttle. If NADPH supply is not concomitantly engineered, this pathway, like the ATP-independent pyruvate to AcCoA routes, has the lowest expected yield for FA produced via FAS (74\% of the theoretical maximum; van Rossum et al. 2016b) due to the imbalance of NADH production and NADPH requirement of FAS. Nevertheless, many oleaginous yeasts, such as Yarrowia lipolytica, which produce large amounts of lipids in the cytosol, rely on this system to provide cytosolic AcCoA (Vorapreeda et al. 2012). The term "shuttle" refers to the fact that AcCoA is formed from pyruvate inside mitochondria via the native PDH complex. Subsequently, the acetate moiety is transferred to oxaloacetate by citrate synthase (CS). The resulting citrate either can enter the TCA cycle inside the mitochondria or be exported to the cytosol to serve as a shuttle for acetyl moieties. A cytosolic ATP citrate lyase (ACL), an enzyme that is not present in S. cerevisiae, is required to form AcCoA and oxaloacetate under the expense of one ATP molecule. The cycle is closed by transporting oxaloacetate back into the mitochondria through a citrate-oxaloacetate antiporter. In a first attempt, the overexpression of a murine ACL in S. cerevisiae led to a slightly increased titer of total FA (Tang et al. 2013). The effect was mostly pronounced during the stationary phase, probably reflecting the redirection of the metabolism toward respiration of the accumulated ethanol.
In the same study, isocitrate dehydrogenase genes $I D H 1$ and $I D H 2$ were deleted to promote the accumulation of citrate; somewhat surprisingly, this intervention did not affect the total content but only the saturation profile of FA, suggesting that ACL activity and/or other factors were rate limiting. Later studies, performed in strains engineered for n-butanol (Lian et al. 2014), hexadecanol (Feng et al. 2015), or mevalonate (Rodriguez et al. 2016) production, showed that the choice of the heterologous enzyme has a certain impact on the pathway efficiency.

In most recent studies that reported the highest titers of FA or their derivatives (Zhou et al. 2016b; Yu et al. 2018), the citrateoxaloacetate shuttle was further optimized and combined with strategies that increase the NADPH supply. The overexpression of ACL alone in a strain that was already engineered to block FA degradation led to a moderate improvement (up to 50\%) of free FA titers, which is consistent with previous studies (Tang et al. 2013; Lian et al. 2014). A further improvement was achieved by introducing a transhydrogenase-like reaction sequence to increase NADPH supply, exemplifying the importance of redox cofactors as a driving force for FA production. In this scheme, a cytosolic malate dehydrogenase (MDH) reduces oxaloacetate to malate (oxidizing $\mathrm{NADH}$ ), and a cytosolic malic enzyme (MAE) subsequently transfers hydrogen to NADP, whereby malate is converted to pyruvate, which then re-enters mitochondria and undergoes a new cycle (Fig. 1). Additional overexpression of the endogenous citrate transporter Ctp1 appeared to have no beneficial effect on the FA production, suggesting that the export of citrate into the cytosol may not have been a limiting factor in this approach. In a follow-up study (Yu et al. 2018), several strategies to enhance the citrateoxaloacetate shuttle were tested. Overexpression of the endogenous (mitochondrial) PDH components to enhance the mitochondrial synthesis of AcCoA did not lead to an increase in FA titers in this study. However, the overexpression of the mitochondrial pyruvate carrier subunits $\mathrm{Mpc} 1$ and $\mathrm{Mpc} 3$ and introduction of a heterologous CS in addition to the native one appeared to increase the flux through the 
citrate-oxaloacetate shuttle, measured as an increase in FA titers by about $20 \%$.

An incremental improvement was achieved by introducing an additional ACL gene from Aspergillus nidulans (a murine ACL was already present in the engineered strain). Simultaneously the isocitrate dehydrogenase Idp2 and the citrate- $\alpha$-ketoglutarate antiporter $\mathrm{Yhm} 2$, which also accepts oxaloacetate instead of $\alpha$-ketoglutarate, were overexpressed. Idp2 was supposed to oxidize citrate to $\alpha$-ketoglutarate and thereby provide additional NADPH for FA synthesis. This, however, can only occur at the expense of the cytosolic citrate and therefore AcCoA pool. Yhm2 overexpression was intended to increase the antiport of citrate with oxaloacetate. Unfortunately, since three genes were introduced at once as compared to the reference strain, their individual contribution to the improvement cannot be disentangled.

A further substantial improvement of FA synthesis in this strain background was achieved by diverting the glucose flux from glycolysis to the oxidative PPP to increase the NADPH pool. To this end, the expression of the phosphoglucose isomerase (PGI1) was downregulated, whereas the endogenous genes encoding PPP enzymes glucose-6-phosphate dehydrogenase (ZWF1), 6-phosphogluconate dehydrogenase (GND1), transketolase (TKL1), and transaldolase (TAL1) were concomitantly overexpressed. A downregulation of the mitochondrial isocitrate dehydrogenase $\mathrm{IDH} 2$ to prevent the degradation of citrate in the TCA led to a further improvement but only in a strain with the upregulated PPP. In its precursor strain, lowering Idh2 activity had no beneficial effect, and this notion is consistent with previous results, obtained in a strain that contained the citrate-oxaloacetate shuttle, but was not engineered for an increased supply of NADPH (Tang et al. 2013). Collectively, these studies show that sufficient reducing power is essential for efficient FA production.

\section{E. Strategies to Manipulate NADPH Level Independently of Precursor Supply Routes}

Besides abovementioned possibilities of overexpressing enzymes that yield NADPH (e.g., Ald6, Zwf1) or creating transhydrogenase cycles (e.g., oxaloacetate-malate-pyruvate), the level of this cofactor can be manipulated through interventions into glycolysis or amino acid metabolism. One possibility to increase the pool of NADPH is bypassing the main source of cellular NADH - the glyceraldehyde-3-phosphate dehydrogenase (GAPDH) reaction-by a NADP-dependent enzyme. The expression of a heterologous non-phosphorylating NADPdependent GAPDH in strains engineered for polyhydroxybutyrate (Kocharin et al. 2013) or FAEE (Shi et al. 2014a) production led to considerable product yield improvements, but not in a strain designed to produce fatty alcohols (d'Espaux et al. 2017). It has to be noted thatbesides different product pathways-the genetic interventions into the carbon metabolism of these strains largely differed, suggesting that the applicability of this strategy is contextdependent. One important consideration is that the activity of a non-phosphorylating GAPDH will reduce the ATP yield of glycolysis, which, as outlined above, ultimately will lead to a reduction of the maximally attainable yield. The implementation of a phosphorylating NADP-dependent GAPDH does not suffer from this downfall, but its utility for production of oleochemicals in S. cerevisiae could not yet be demonstrated (d'Espaux et al. 2017). A complementary approach is reducing the NADPH consumption through competing pathways. Based on the observation that a large proportion of NADPH is oxidized by NADP-dependent glutamate dehydrogenase Gdh1, d'Espaux et al. (2017) demonstrated that a deletion of the $G D H 1$ gene is beneficial for fatty alcohol production.

\section{F. Engineering Malonyl-CoA Supply}

MalCoA, the extender unit for FA synthesis via FAS, is synthesized by carboxylation of AcCoA in an ATP-dependent manner by the endogenous enzyme AcCoA carboxylase (Acc1). In some studies, the native form of Acc1 was overexpressed to boost the production of FA derivatives, which indeed led to higher product titers (Shin et al. 2012; Runguphan and Keasling 2014). As an alternative approach, Wang 
et al. (2014b) overexpressed a plant MalCoA synthetase, which ligates malonic acid and CoA to generate MalCoA directly. This improved the productivity of strains engineered for polyketide production and increased the total FA amount. Since the native Accl is known to be inactivated by phosphorylation by the kinase Snf1 upon glucose depletion, different strategies were developed to circumvent this regulatory mechanism, including relocalization of the mitochondrial ACC from S. cerevisiae (Hfa1) into the cytosol (d'Espaux et al. 2017) and site-directed mutagenesis to prevent phosphorylation (Choi and Da Silva 2014; Shi et al. 2014b). All of them led to remarkable improvements in yields of MalCoA-derived products.

The specific activity of the phosphorylation-resistant mutant Acc1 ${ }^{\text {S1157A }}$ was higher compared to wild-type Accl even when measured in extract from glucosegrown cells (Choi and Da Silva 2014), suggesting that the protein might be partly phosphorylated also on this carbon source (not only on ethanol). In another study, a second (in silico predicted) phosphorylation site (S659) was mutated to alanine in addition to S1157, which led to an increase in specific activity and total FA yields compared to a control harboring the S1157A mutation alone (Shi et al. 2014b). Considering that Snf1 is activated by AMP (Sanz 2003), we hypothesize that phosphorylation resistance may become even more relevant on glucose-grown cells when the PDH bypass, which produces AMP in the ACS reaction (Fig. 1), is overexpressed.

Another important consideration for strain design is the sensitivity of FA chain lengths to the AcCoA/MalCoA ratio. Elevated concentration of MalCoA is known to favor the elongation of the FA carbon chains by FAS (Sumper et al. 1969). Indeed, the expression of $\mathrm{Accl}^{\text {S1157A }}$ (Choi and Da Silva 2014; Hofbauer et al. 2014; Besada-Lombana et al. 2017) and $\mathrm{Accl}^{\text {S1157A, S659A }}$ (Zhou et al. 2016b) led to a higher proportion of longer FA chains. This demonstrates that, especially if tight chain length control is desired, the relative abundance of the precursor molecules must be subtly balanced. By affecting the lipid composition of the membranes, the ACC hyperactivity not only changes the product profiles but also appears to negatively influence cell growth, which was attributed to an imbalanced synthesis of long-chain FA or depletion of intermediates (Shi et al. 2014b). On the other hand, the expression of $A c c 11^{\mathrm{S1157A}}$ was shown to improve the resistance of yeast to medium-chain FA (see Sect. IV.B). All these observations demonstrate that balancing the ACC activity is a non-trivial task and several factors such as the AcCoA abundance, product chain length, and toxicity must be taken into account. The development of product-specific biosensors (see Sect. IV.C) will accelerate the screening of strains producing the desired chain length in a highthroughput manner, which will greatly facilitate the balancing of AcCoA and MalCoA supply.

To summarize, the endogenous supply of cytosolic precursors (AcCoA and MalCoA), redox cofactors (NADH or NADPH), and energy equivalents (ATP) via the PDH bypass is not sufficient for production of FA and derivatives in S. cerevisiae beyond the proof-ofconcept level. Although there are no systematic studies comparing the FA yields in dependence on different precursor supply routes, stoichiometric analyses (van Rossum et al. 2016b) and experimental data outlined above show that there is no "one best" precursor supply pathway valid for all AcCoA-derived products. Since the choice of the FA elongation system (FAS vs. reverse $\beta$-oxidation; see Sect. III) dictates the redox cofactor requirement, the appropriate precursor supply pathway must be chosen accordingly. Moreover, to minimize the amount of carbon that needs to be diverted for energy supply, the native PDH bypass must be fully replaced by a combinatorial precursor supply configuration, as predicted by stoichiometric analyses for different model compounds (van Rossum et al. 2016b) and experimentally shown for the isoprenoid farnesene (Meadows et al. 2016). Considering that very promising improvements in FA productivity, reaching total yields up to $30 \%$ of the theoretical maximum, have been recently achieved (Yu et al. 2018), it is conceivable that similar combinatorial approaches can lead to further advances in the production of oleochemicals. 


\section{Strategies for Chain Length Control}

The majority of microbially synthesized FAs are 14-20 carbon atoms in length (referred to as long-chain FA). Mainly saturated and monounsaturated FA with 16 or 18 carbon atoms are synthesized in S. cerevisiae (Cottrell et al. 1986). Engineering microbes like E. coli, S. cerevisiae, or oleaginous yeasts (e.g., Yarrowia lipolytica) toward FA and FA derivatives with alternating chain length is desired due to their compelling properties as biofuels; for the cosmetic, healthcare, and pharmaceutical industries; or as platform compounds (Lennen and Pfleger 2013).

In microorganisms however, biosynthesis of short- $(\leq \mathrm{C} 4)$, medium- (C6-C12), or very long- $(\geq$ C22) chain FA is rare and low in quantity. For this reason strategies for chain length control through a focus on the main anabolic (FA biosynthesis) and catabolic ( $\beta$-oxidation) pathways have been developed.

\section{A. Manipulation of the Natural Fatty Acid Biosynthesis and Elongation Machinery}

Fatty acid biosynthesis follows a conserved chemistry among different types of FAS enzymes which differ in their general organization. Type I FAS systems (mainly eukaryotic) carry all catalytic domains in one multifunctional complex (Schweizer and Hofmann 2004). However, fungal type I FAS greatly differ from animal type I FAS in their architecture (Leibundgut et al. 2008).

In type I systems, FA biosynthesis is initiated by the transfer of the starter unit AcCoA to the acyl-carrier protein (ACP) by the acetyltransferase (AT) domain of FAS. Subsequently, the ACP domain delivers the acetyl residue to the ketosynthase (KS) domain. Similarly, the extension unit MalCoA is loaded onto the free ACP by the malonyl-palmitoyl transferase (MPT) domain. Acetyl and malonyl moieties are condensed by the KS domain to a $\beta$ ketoacyl intermediate, which is then further processed. The $\beta$-ketoacyl intermediate is reduced by the ketoacyl reductase (KR) domain to form 3-hydroxyacyl-ACP, which is dehydrated by the dehydratase (DH) domain and eventually reduced by the enoyl reductase (ER) domain to a saturated acyl chain containing two additional carbon atoms. The reductive steps at the KR and ER domains are strictly NADPH-dependent. After completion of one cycle, the acyl chain is either condensed with another malonyl moiety for further elongation or released by the MPT domain as a CoA-bound thioester in fungal FAS (Lomakin et al. 2007). After release of acyl-CoA esters from the FAS complex, thioesterases (TE) cleave the thioester bond between the acyl chain and CoA to release free FA.

As a strategy for enrichment of short- and medium-chain FA, expression of short- and medium-chain-specific TE for early termination of FA biosynthesis has been established in multiple studies (Leber and Da Silva 2014; Fernandez-Moya et al. 2015; Xu et al. 2016; Zhu et al. 2017b). Combined with fungal FAS enzymes, soluble TE only have limited or no access to FAS-bound acyl-CoA or acyl-ACP esters, and protein engineers have therefore developed strategies to locate TE within the FAS complex. For instance, some type I FAS (e.g., from Rhodosporidium toruloides and Aplanochytrium kerguelense) harbor two redundant ACP domains, which are located inside of the FAS scaffold. It is therefore possible to replace one of them by a short-chain TE to enable a direct access to acyl intermediates inside the reaction chamber. The implementation of this strategy proved to be more efficient than the expression of free TE and increased short- and medium-chain FA production by 3 to 15-fold (Zhu et al. 2017b). In Y. lipolytica FAS, replacement of the MPT domain by a short- and medium-chain-specific TE resulted in an increase of up to $29 \%$ of C12-C14 FA of the total FA content (Xu et al. 2016).

As an alternative approach, the yeast FAS has been replaced by non-fungal enzymes. Human FAS (hFAS) carries its own TE domain, which releases the acyl moieties directly from the ACP (Leibundgut et al. 2008) and has a more flexible structure than yeast FAS (Brignole et al. 2009). Expression of hFAS, in which its own TE domain was deleted, in com- 
bination with the short-chain-specific TE CpFatB1 (Cuphea palustris) or TEII (Rattus norvegicus) as free proteins enabled the production of C6-C10 FA (Leber and Da Silva 2014). Substituting the TE domain of hFAS by TEII in the same polypeptide chain substantially increased the production of short- and medium-chain FA.

In contrast to type I FAS, the catalytic domains of the bacterial type II FAS system are expressed as single enzymes. This allows a simpler manipulation of the subunits, and acyl intermediates are freely accessible for TE. Expression of the E. coli system (acpS, acpP, fabB, fabD, fabG, fabH, fabI, fabZ) in combination with the TE fatB from Ricinus communis in a FAS-deficient $S$. cerevisiae strain significantly increased the total FA titer and shifted the FA profile toward C14 FA production (FernandezMoya et al. 2015).

A minimally invasive strategy to rewrite the chain length control relies on rational engineering of yeast FAS by site-directed mutagenesis. For instance, an early release of shorter acyl chains (C6-C10 or C14) was achieved by introducing bulky residues like tryptophan into the KS domain to shorten the acyl-ACP binding channel in S. cerevisiae (Gajewski et al. 2017; Zhu et al. 2017b) or Y. lipolytica (Rigouin et al. 2017) FAS. Furthermore, it was shown that decreasing the affinity of the MPT domain for malonyl moieties by introducing one point mutation disfavors the chain elongation and promotes the release of shorter-chain (C6C10) FA (Gajewski et al. 2017). One additional mutation was introduced into the AT domain for a more frequent priming with $\mathrm{AcCoA}$, as this was expected to shift the product profile toward shorter chains; however, this modification proved only effective in combination with mutations in the MPT and/or KS domain. Single or multiple mutations in the three domains caused different ratios of $\mathrm{C} 6-\mathrm{C} 10 \mathrm{FA}$, with some of the mutants exhibiting a considerable specificity, e.g., for C8 FA (Gajewski et al. 2017). Thus, rational engineering of FAS holds a great promise for narrowing down the product profile to the desired chain length.

Very long-chain FAs (C22-C26) are present in $S$. cerevisiae at low abundance (Welch and
Burlingame 1973) but are essential compounds, e.g., for sphingolipids (Oh et al. 1997). After their release from the FAS complex, FAs are elongated at the ER membrane from (longchain) acyl-CoA precursors. Elongases with different product specificities (Elo1, Elo2, and Elo3) carry out the elongation of acyl-CoA intermediates with MalCoA. Elo1 elongates medium- and long-chain compounds (C14C16) to C18 FA, Elo2 elongates compounds up to $22 \mathrm{C}$ atoms, and Elo3 elongates compounds up to $26 \mathrm{C}$ atoms (Toke and Martin 1996; Oh et al. 1997). Reminiscent of cytosolic FA biosynthesis, two reductions and one dehydration step are necessary to complete very long-chain FA synthesis. To selectively increase the pool of C22, overexpression of ELO2 and deletion of ELO3 are necessary (Yu et al. 2017), whereas overexpression of only ELO3 is sufficient for enrichment of C26 FA (Wenning et al. 2017). Alternatively, FAS I from mycobacteria has been shown to naturally generate FA with 2226 carbon atoms (Kaneda et al. 1995). Expression of FAS I from Mycobacterium vaccae in an Elo2-/Elo3-deficient strain increased the C22 FA pool by fourfold (Yu et al. 2017).

\section{B. Reversal of $\beta$-Oxidation as an Orthogonal Pathway for Fatty Acid Biosynthesis}

The $\boldsymbol{\beta}$-oxidation cycle naturally is an FA degradation pathway (for review see Hiltunen et al. 2003). In each turn of the cycle, FAs are truncated by removing two carbon atoms from the FA chain, thereby generating AcCoA.

Reversing all reactions of the $\beta$-oxidation can consequently be used as an alternative synthetic pathway for FA production. The single reactions of the $\beta$-oxidation are equilibriumbalanced, and the functionality of the enzymes in the reverse direction has been demonstrated (Dellomonaco et al. 2011; Clomburg et al. 2012). The reverse $\beta$-oxidation pathway starts with the condensation of two AcCoA to acetoacetyl-CoA by a thiolase, followed by the reduction of the $\beta$-ketogroup to 3-hydroxyacyl-CoA by a reductase/dehydrogenase, dehydration to transenoyl-CoA by a hydratase/dehydratase, and a final reduction by another reductase/dehydro- 
genase, yielding butyryl-CoA. In this cycle, $\mathrm{NADH}$ or NADPH can serve as electron donors for the reductive steps (depending on the choice of heterologous enzymes). By iterating this reaction sequence, the acyl chain can be elongated by two carbon atoms per cycle. Depending on the desired chain length and functional groups of the products, the reverse $\beta$-oxidation can be terminated through different enzymes. For instance, TE can release free FA from the CoA-bound form, and aldehyde/ alcohol dehydrogenases can reduce the acylCoA esters to n-alcohols (see Sect. V).

Elongation beyond a length of four carbon atoms requires multiple turns of reverse $\beta$-oxidation, which is hampered by a competition between the thiolase and the termination enzymes. Thus, although the synthesis of minor amounts of longer-chain FA via reverse $\beta$-oxidation could be demonstrated at the proof-of-concept level in E. coli, the product yields gradually decreased with each iteration of the cycle (Dellomonaco et al. 2011; Clomburg et al. 2012). Follow-up studies therefore focused on improving the selectivity of reverse $\beta$-oxidation for longer-chain products. To this end, the core pathway was expanded by thiolases that accept acyl-CoA intermediates with various chain lengths, such as the $\beta$-ketothiolase BktB, which proved suitable to produce $\mathrm{C} 6-\mathrm{C} 10 \mathrm{com}$ pounds in multiple studies (Dekishima et al. 2011; Clomburg et al. 2015; Kim et al. 2015). The chain length specificity of termination enzymes (i.e., TE for free FA) was shown to be the second key determinant for selective production of longer acyl chains (Clomburg et al. 2015; Kim and Gonzalez 2018). To prevent a premature termination of the cycle, the deletion of endogenous TE genes was critical in E. coli strains. These observations are likely transferrable to $S$. cerevisiae. To provide sufficient carbon and reducing equivalents, heterologous reverse $\beta$-oxidation pathways were expressed in the yeast cytosol. As a proof of concept, nbutanol production, for the synthesis of which only one functional turn of the cycle is needed, has been targeted. Using a variety of enzymes from different organisms and in combination with different precursor supply pathways (see above), titers of up to $1 \mathrm{~g} \mathrm{~L}^{-1}$ could be reached (Lian and Zhao 2015; Schadeweg and Boles 2016a, b). Less effort has been dedicated to the production of medium-chain products in $S$. cerevisiae so far, but the feasibility could be demonstrated by combining reverse $\beta$-oxidation with a medium-chain-specific TE, $\mathrm{CpFatB1}$, thereby enabling the production of C6-C10 FA, albeit at a low yield (Lian and Zhao 2015). It is certain that by transferring the expanded enzyme toolbox developed in $E$. coli (Kim and Gonzalez 2018) and fine-tuning the expression of endogenous TE, many of which were recently characterized (e.g., Kruis et al. 2018), the production of short- and medium-chain FA via reverse $\beta$-oxidation will be further optimized in S. cerevisiae in the future.

Directly compared, canonical FA biosynthesis and reverse $\beta$-oxidation both bear certain advantages and drawbacks. The most obvious advantage of FA biosynthesis is a rather strict chain length control that can be easily manipulated by protein engineering of FAS, as outlined above, whereas specific production of longer carbon chains via reverse $\beta$-oxidation is still very challenging. While the FAS functional modules operate as a perfectly synchronized machine within one macromolecular complex, it is difficult to fine-tune the activity of individual reverse $\beta$-oxidation enzymes. At the current stage of technology, reverse $\beta$-oxidation can compete with FA biosynthesis in the product range of up to ten carbon atoms. On the other hand, FA biosynthesis is energetically more expensive than reverse $\beta$-oxidation, since each elongation cycle by FAS requires one MalCoA that is synthesized at the expense of one ATP molecule. In contrast, reverse $\beta$-oxidation has the advantage of using only AcCoA for elongation steps, which also makes it insensitive to the AcCoA/MalCoA ratio, a parameter that has a significant influence on the chain length control by FAS and overall cellular fitness. Whereas the reverse $\beta$-oxidation can be designed to use the easily available NADH, FAS is strictly dependent on the less abundant NADPH. Thus, the redox cofactor supply of reverse $\beta$-oxidation requires far less interventions into the central 
carbon metabolism compared to the FA biosynthesis route and offers a larger choice of potential precursor supply routes (see Sect. II, Fig. 1).

\section{Chassis Engineering}

To achieve high FA production levels in S. cerevisiae, several obstacles have to be addressed within the chosen production pathway. Blocking the degradation of FA has been achieved through the disruption of different enzymes involved in $\beta$-oxidation. An issue that remains to be tackled is the toxicity of some FA. We will discuss different methods that have or could be employed to engineer more robust chassis. Furthermore, several biosensor systems have been developed, which can be used in high-throughput screenings to further speed up the search for best performing strains.

\section{A. Elimination of By-product Formation and Fatty Acid Degradation}

To increase FA pools, it is important not only to increase precursor pools (see Sect. II, Fig. 1) but also to downregulate competing pathways as well as to prevent degradation through specific gene deletions or overexpressions.

\section{Prevention of $\beta$-Oxidation}

The degradation, i.e., $\beta$-oxidation, of the already synthesized free FA or their activated forms, the acyl-CoAs, takes place in the peroxisomes (Hiltunen et al. 2003; van Roermund et al. 2003). In S. cerevisiae, $\beta$-oxidation of saturated FA is a cyclic mechanism mainly catalyzed by the three enzymes Pox1, Fox2, and Pot1 (Hiltunen et al. 2003). Pox1 is an acyl-CoA oxidase catalyzing the first step of the degradation of an acyl-CoA molecule. The dehydrogenated intermediate is then modified by the multifunctional enzyme Fox2, which acts as an enoyl-CoA hydratase as well as a 3-hydroxyacyl-CoA dehydrogenase, followed by thiolytic cleavage through Pot1, a 3-ketoacyl-CoA thiolase. These cyclic steps are repeated until the FA molecule is completely degraded (Hiltunen et al. 2003).

Prior to peroxisomal $\beta$-oxidation, free FAs are activated to their acyl-CoA form. In S. cerevisiae, this reaction can be catalyzed by five enzymes, Faa1, Faa2, Faa3, Faa4, and Fat1 (Black and DiRusso 2007). Long-chain FAs are primarily activated by Faal and Faa4 in the cytosol (Scharnewski et al. 2008) and transported into the peroxisomes by the heterodimeric ATP-binding cassette transporter Pxal/ Pxa2 (Hettema et al. 1996). Fat1 is a multifunctional enzyme, which imports long-chain FA into the peroxisomes and also has an acylCoA synthetase activity for very long-chain FA (Zou et al. 2002). Medium-chain FAs, on the other hand, are assumed to enter peroxisomes by passive diffusion or spontaneous flipping and are then activated by the peroxisomal Faa2 (Knoll et al. 1994; Hettema et al. 1996; Hettema and Tabak 2000). The role of Faa3 is not entirely clear yet. It showed some activity on very long-chain FA; however, its in vitro activity on long-chain FA was much lower than that of Faa1 and Faa2 (Johnson et al. 1994; Knoll et al. 1994).

The effects of disrupted FA activation, $\beta$ oxidation, or both, on FA titers have been studied extensively. In $S$. cerevisiae strains engineered for increased FA production, a positive effect on free FA titers was detected when deleting FAA1 and FAA4 separately, as well as together; however, only minimal increases were observed when deleting POX1 (Li et al. 2014; Runguphan and Keasling 2014). Another group found that the two triple deletion mutants $\Delta$ faa $2 \Delta$ pxa $1 \Delta$ pox 1 and $\Delta$ faa $1 \Delta f a a 4 \Delta f a t 1$ produced more free FA than the original strain (Leber et al. 2015). The combination of all six knockouts in one strain showed the highest titers with $1.3 \mathrm{~g} \mathrm{~L}^{-1}$ free $\mathrm{FA}$, corresponding to $18 \%$ of the maximum theoretical yield (Leber et al. 2015).

Apart from these rather general $\beta$-oxidation disruptions, there have also been approaches for a chain length-specific disruption of $\boldsymbol{\beta}$-oxidation, involving FAA2, PEX11, and ANT1 (Leber et al. 2016). Pex11 is thought to transport short- and medium-chain FA into the peroxisomes, where they are activated by 
Faa2 (Knoll et al. 1994; van Roermund et al. 2000). Ant1 is an adenine nucleotide transporter, which exchanges AMP by ATP across the peroxisomal membrane, thereby providing the energy for the acyl-CoA activation of the free FA (van Roermund et al. 2001). To decrease $\beta$-oxidation of specifically shortchain FA, a strain with three knockouts $(\Delta$ faa $\Delta$ ant $1 \Delta$ pex 11$)$ was generated, which displayed a much stronger increase in hexanoic and octanoic acid levels compared to the "full" $\beta$-oxidation-deficient $\Delta$ faa $2 \Delta p x a 1 \Delta p o x 1$ mutant (Leber et al. 2016). This observation was attributed to the disruption of the $\mathrm{AcCoA}$ recycle mechanism in the latter strain (Leber et al. 2016). Overall, the ideal combination of fatty acyl-synthetase and $\beta$-oxidation gene knockouts must be evaluated carefully, depending on the desired chain length of the final FA. Nevertheless, most reported gene knockouts of this pathway have turned out to be beneficial for increasing FA titers (Buijs et al. 2015; Zhou et al. 2016b).

\section{Disruption of Triacylglycerol and Steryl Ester Synthesis}

A competing pathway that can decrease FA levels is the incorporation into storage lipids, primarily triacylglycerols (TAGs) or steryl esters (SEs). In S. cerevisiae, TAGs are synthesized from diacylglycerols and fatty acyl-CoAs by the acyltransferases Dga1 and Lro1 (Oelkers et al. 2000, 2002). Steryl ester synthesis of sterols and fatty acyl-CoAs is catalyzed by the acylCoA:sterol acyltransferases Are1 and Are2 (Yang et al. 1996). In engineered yeast strains, the knockout of one or several of these four TAG/SE genes has led to increased production of FA derivatives such as 1-hexadecanol (Tang and Chen 2015), fatty alcohols (d'Espaux et al. 2017; Tang et al. 2017), and FAEEs (ValleRodríguez et al. 2014). In another strain optimized for FA production (by disruption of $\beta$ oxidation and acyl-CoA-activating enzymes), TAG synthesis was increased by overexpressing Dgal. This was combined with an overexpression of the lipid recycle via the triacylglycerol lipase Tgl3, leading to $2.2 \mathrm{~g} \mathrm{~L}^{-1}$ extracellular free FA (Leber et al. 2015).

\section{B. Engineering Yeast Product Tolerance and Excretion}

Some FA as well as some of their derivatives are toxic to S. cerevisiae. In general, yeast is a robust cell factory that can sense stress and adapt its metabolism accordingly. However, to avoid loss of cell viability and ensure economically competitive yields, yeast strains are needed, which are tolerant to high product concentrations even at low pH (Deparis et al. 2017). Over the last years, much progress has been made to understand the underlying principles of the toxicity of these compounds, and random as well as rational approaches have been applied to improve yeast robustness and thereby product yields.

\section{Toxicity Mechanisms and Transport of Fatty} Acids and Derivatives

FAs that are toxic to S. cerevisiae include hexanoic, octanoic, and decanoic acids (LafonLafourcade et al. 1984; Viegas et al. 1989; Liu et al. 2013). At acidic pH, which is the common fermentation condition, the undissociated FA can enter the cells by passive diffusion (Viegas 1997). In the neutral cytosol, they dissociate, thereby causing a decrease of the intracellular $\mathrm{pH}$ and an accumulation of the toxic anions (Viegas et al. 1989, 1998; Viegas 1997). Furthermore, they disturb the plasma membrane integrity, causing changes in membrane composition, permeability, and fluidity (Alexandre et al. 1996; Legras et al. 2010; Liu et al. 2013). To generate strains with higher tolerance, it is important to ensure a rapid transport/secretion of FA out of the cell, as well as to avoid their re-entrance into the cells. A transcriptome analysis of octanoic- and decanoic acidstressed cells, respectively, revealed a partly overlapping response mechanism, which was similar to an oxidative stress response, but it also showed a compound-specific activation of 
genes encoding transcription factors and transporters (Legras et al. 2010). The role of the identified transporters Tpol and Pdr12 in octanoic and decanoic acid efflux was further analyzed in growth tests with knockout mutants (Legras et al. 2010). An overexpression of such efflux-involved transporters is one approach that could increase product yields-in the case, that secretion is a bottleneck. For example, overexpression of Pdr12 was shown to increase the secretion of short branched-chain FA at early time points of production (Yu et al. 2016). However, transporter overexpression can also have unwanted side effects, such as slower growth, depleting ATP from other cellular processes or altering the plasma membrane composition, as was hypothesized based on other Pdr12 overexpression studies (Nygård et al. 2014). Due to a lack of yeast efflux pumps known to specifically transport FA and their derivatives, another viable approach is the screening of heterologous transporters. In a recent study, 12 human or Arabidopsis thaliana (putative) transporters were screened, and one of them, human FATP1, improved overall cell fitness and fatty alcohol (Hu et al. 2018) as well as 1-alkene (Zhou et al. 2018) production and secretion in S. cerevisiae. For alkane resistance of $S$. cerevisiae, endogenous efflux pumps, namely, Snq2 and Pdr5, as well as heterologous transporters, namely, $Y$. lipolytica $\mathrm{ABC} 2$ and $A B C 3$, were shown to have a positive effect (Chen et al. 2013a; Ling et al. 2013).

\section{Methods for Increasing Yeast Robustness}

To avoid the re-entrance of the products, the robustness of the plasma membrane needs to be improved. Octanoic acid, for instance, disrupts the plasma membrane composition, leading to membrane leakage and cell death (Legras et al. 2010; Liu et al. 2013). This effect was reduced by rationally engineering plasma membrane composition through increasing the oleic acid content, either by external supply (Liu et al. 2013) or by expression of a mutated AcCoA carboxylase, Acc1 ${ }^{\text {S1157A }}$ (Besada-
Lombana et al. 2017). The increase in the average chain length of membrane FA, as well as higher cis-monounsaturated FA levels, was shown to provide higher tolerance to toxic FA (Liu et al. 2013; Besada-Lombana et al. 2017).

As tolerance is usually a complex phenotype, which cannot solely be improved by rational engineering of single genes, adaptive laboratory evolution (ALE) is an interesting alternative. In an ALE experiment, a strain is grown over many generations, with increasing concentrations of the toxic compound, for selection of cells with an enhanced tolerance phenotype. Causal mutations in the final strain are identified by whole genome sequencing (Dragosits and Mattanovich 2013; Mans et al. 2018). When performed with E. coli, an evolved strain not only showed higher tolerance to octanoic acid but also produced higher titers, which was attributed to changes in membrane composition and fluidity (Royce et al. 2015). A similar approach would be possible for S. cerevisiae, for which ALE has been performed successfully, for example, for increased resistance to high temperatures (Caspeta et al. 2014) and acetic acid (González-Ramos et al. 2016) or alcohol tolerance (González-Ramos et al. 2013; Davis López et al. 2018), but not for short- or medium-chain FA tolerance.

A method that can lead to similar results as ALE was termed global transcription machinery engineering, gTME (Alper et al. 2006). It can induce a remodeling of the transcriptome and therefore target polygenic traits, such as tolerance. gTME relies on the random mutagenesis of a transcription factor that regulates the transcription of several genes and was successfully applied to enhance $S$. cerevisiae tolerance to ethanol (Alper et al. 2006), as well as to adapt to growth on lignocellulosic hydrolysates through improved xylose utilization (Liu et al. 2011). When using this method in combination with an appropriate selection for mutants with increased growth in the presence of the toxic FA, it could be a valuable tool for future tolerance engineering but has so far not been applied to S. cerevisiae for FA tolerance. 


\section{Dynamic Pathway Control and Biosensors}

The metabolic engineering of yeast to produce a desired compound can result in an imbalanced metabolism due to the disruption of a tightly regulated, complex biological system, which has evolved for growth and survival. However, inherent control components of this system, such as promoters, transcription factors, or gene copy number, can be utilized for a dynamic pathway control, thereby minimizing metabolic imbalances and increasing product titers (Michener et al. 2012; Schallmey et al. 2014; Shi et al. 2018).

\section{Altering Gene Expression Level and Timing}

The level and timing of pathway gene expression is crucial in metabolic engineering and can be adjusted, for instance, by using constitutive promoters of different strengths or promoters that are inducible/repressible (Da Silva and Srikrishnan 2012). This strategy was applied successfully, for example, to increase 1-alkene production and secretion in S. cerevisiae. By replacing the strong eTDH3 promoter for expression of the membrane-bound enzyme PfUndB with the GAL7 promoter, which is activated upon glucose depletion, 1-alkene production was decoupled from growth (Zhou et al. 2018). The same strategy, i.e., to separate growth from production by expressing pathway genes under the control of carbon sourcedependent promoters, was also effective in increasing docosanol production (Yu et al. 2017). A similar approach was applied for $S$. cerevisiae short- and medium-chain FA production, again with a glucose-repressed promoter. Here, FAS1 and FAS2 were expressed on a low copy plasmid under control of the alcohol dehydrogenase II promoter of $S$. cerevisiae, $\mathrm{pADH} 2$, leading to increased short- and medium-chain FA titers (Gajewski et al. 2017). In another approach, several promoters of different strengths were tested for downregulating the expression of IDH2 (carbon flux redistribution into free FA) and PGI1 (increasing NADPH supply), respectively, as the deletion of either gene led to growth defects. The expression of the genes was reduced by using weaker promoters, leading to increased long-chain FA production in S. cerevisiae (Yu et al. 2018). These examples underpin the importance of an appropriate control of gene expression and have much potential to be further exploited for increasing short- and medium-chain FA production-especially as promoters and terminators of different strengths have been described in great detail in recent years (Alper et al. 2005; Da Silva and Srikrishnan 2012; Curran et al. 2013; Lee et al. 2015).

\section{Biosensors}

Currently, the analysis of short- and mediumchain FA titers is time-consuming and laborious and is usually achieved through chromatography-based methods. A more rapid and convenient alternative are biosensors. They detect the concentration of a molecule-ideally over a wide range of concentrations - and transform it into an easily detectable, quantifiable output, such as growth rate or fluorescence, eventually enabling highthroughput screenings, as depicted in Fig. 2 (Michener et al. 2012; Schallmey et al. 2014; Shi et al. 2018). One example of in vivo biosensors is transcription factors, which, in nature, are inevitable for the dynamic control of gene expression. Once their inducing molecules and target promoters are known, transcription factors can be used in metabolic engineering to regulate production pathway expression or even live-monitor titers. Several bacterial transcription factor-based systems have been adapted to yeast (Teo et al. 2013; Li et al. 2015; Skjoedt et al. 2016; Wang et al. 2016), especially dynamic sensor-regulator systems of the FA intermediate MalCoA (Johnson et al. 2017). The prokaryotic transcription factor-based FapR-fapO system has been engineered in $S$. cerevisiae and enabled the sensing of intracellular MalCoA levels (Li et al. 2015; David et al. 2016). This system has been expanded to dynamically control production by coupling the expression of 3-hydroxypropionic acid path- 
a. FapR-fapO, FadR

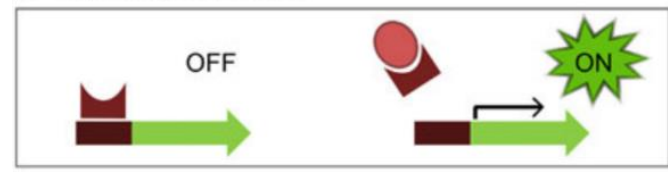

b. War1-pPDR12

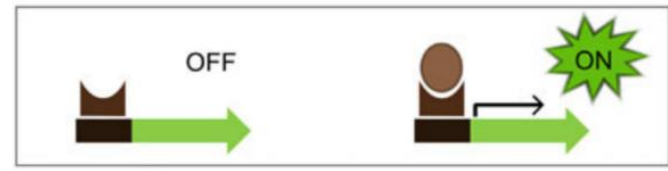

c. GPCR

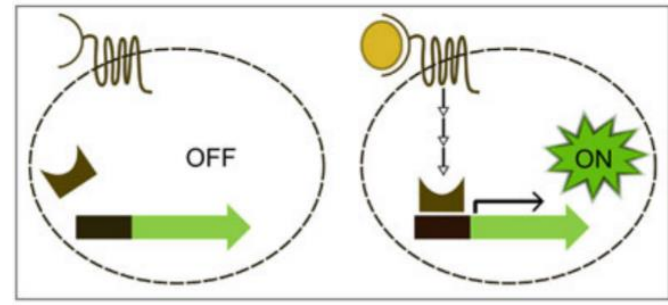

Fig. 2 Yeast biosensors developed for high-throughput screenings of fatty acids or pathway intermediates. (a) FapR and FadR are bacterial repressors (OFF state) which have been adapted to yeast for biosensing. Once they are bound by MalCoA (FapR) or FA/fatty acyl-CoA (FadR), expression is enabled, e.g., of a downstream-located fluorescent protein (ON state). (b) The yeast endogenous PDR12 promoter ( $\mathrm{p} P D R 12)$ is regulated by the transcription factor Warl that is thought to constitutively bind to the promoter. Warlp

way genes to the intracellular MalCoA level (David et al. 2016). Another bacterial regulation system is based on the transcriptional repressor FadR, which is repressed in the presence of FA/ fatty acyl-CoAs and cannot bind to specific operator sites (Zhang et al. 2012). This property was exploited to monitor FA levels in S. cerevisiae by coupling it to synthetic yeast promoters and GFP expression (Teo et al. 2013). While these MalCoA sensor-regulator systems represent a promising method to fine-tune intracellular precursor supply, systems that directly sense the final, excreted FA, constitute another valuable tool for metabolic engineering. However, reports about such biosensors are scarce to date. The first short- and medium-chain FA yeast biosensor was shown to respond to octanoic and decanoic acid via heterologous G-protein-coupled receptors that were linked to the
MalCoA, FA fatty acyl-CoA

$\mathrm{FA}(\mathrm{C} 6, \mathrm{C} 7, \mathrm{C} 8)$

$\mathrm{FA}(\mathrm{C} 8, \mathrm{C} 10)$

high-throughput screenings (e.g. FACS)

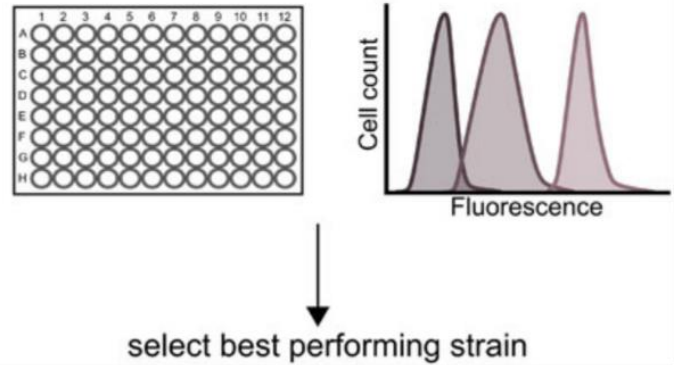

changes to an active form upon $\mathrm{C} 6, \mathrm{C} 7$, or $\mathrm{C} 8 \mathrm{FA}$ presence. (c) A G-protein-coupled receptor responsive to $\mathrm{C} 8$ and $\mathrm{C} 10 \mathrm{FA}$ was coupled to the yeast mating pathway for signaling and GFP expression as a quantifiable output. For high-throughput screenings with biosensors, high-throughput cultivation platforms and/or screening methods, such as fluorescence-activated cell sorting (FACS), are needed to select best performing strains

yeast mating pathway for signaling and GFP expression as a concentration-dependent output (Mukherjee et al. 2015). Besides the rather low linear and dynamic ranges of this biosensor, it was not proven to be functional in culture broth-an indispensable feature for an applicable biosensor. Another biosensor, which was developed for para-hydroxybenzoic acid, was based on the weak acid-inducible PDR12 promoter which is regulated by the transcription factor Warl (Williams et al. 2017). Our group recently adapted this system for the sensing of hexanoic, heptanoic, and octanoic acid reaching high linear and dynamic ranges (Baumann et al. 2018). This biosensor can sense short- and medium-chain FA in $S$. cerevisiae culture broth, which facilitates the monitoring of end-product concentrations and opens the path to high-throughput screenings 
of producer strain libraries. High-throughput screening, with the help of appropriate cultivation facilities, fluorescence-activated cell sorting (FACS), or microfluidics, permits the rapid screen of hundreds and thousands of strains to identify best performing cells (Becker et al. 2004; Dietrich et al. 2010; Wang et al. 2014a). High-throughput screening technologies have the potential to substantially speed up metabolic engineering efforts and strain selection in the next years (Schallmey et al. 2014).

\section{S. cerevisiae as a Production Platform for Fatty Acid Derivatives}

Several biosynthetic pathways have recently been engineered to produce a broad spectrum of FA derivatives in various microbes. In this part, we will give a brief overview of some recently reported biosynthetic pathways in $S$. cerevisiae to produce FA esters, fatty alcohols, fatty aldehydes, and alkanes/alkenes as well as dicarboxylic acids. FA-derived compounds can be synthesized on the one hand from fatty acylCoA, the end product of the yeast FA biosynthesis, and on the other hand from free FA, which arise after the cleavage of CoA by endogenous yeast TE. In contrast to acyl-CoA-the biosynthesis of which is tightly regulated by feedback inhibition-free FA can be accumulated to much higher levels in yeast (Foo et al. 2017; Teixeira et al. 2017). Both precursors can be processed by several heterologous enzymes as illustrated in Fig. 3. All downstream pathways have in common that the length of the acyl

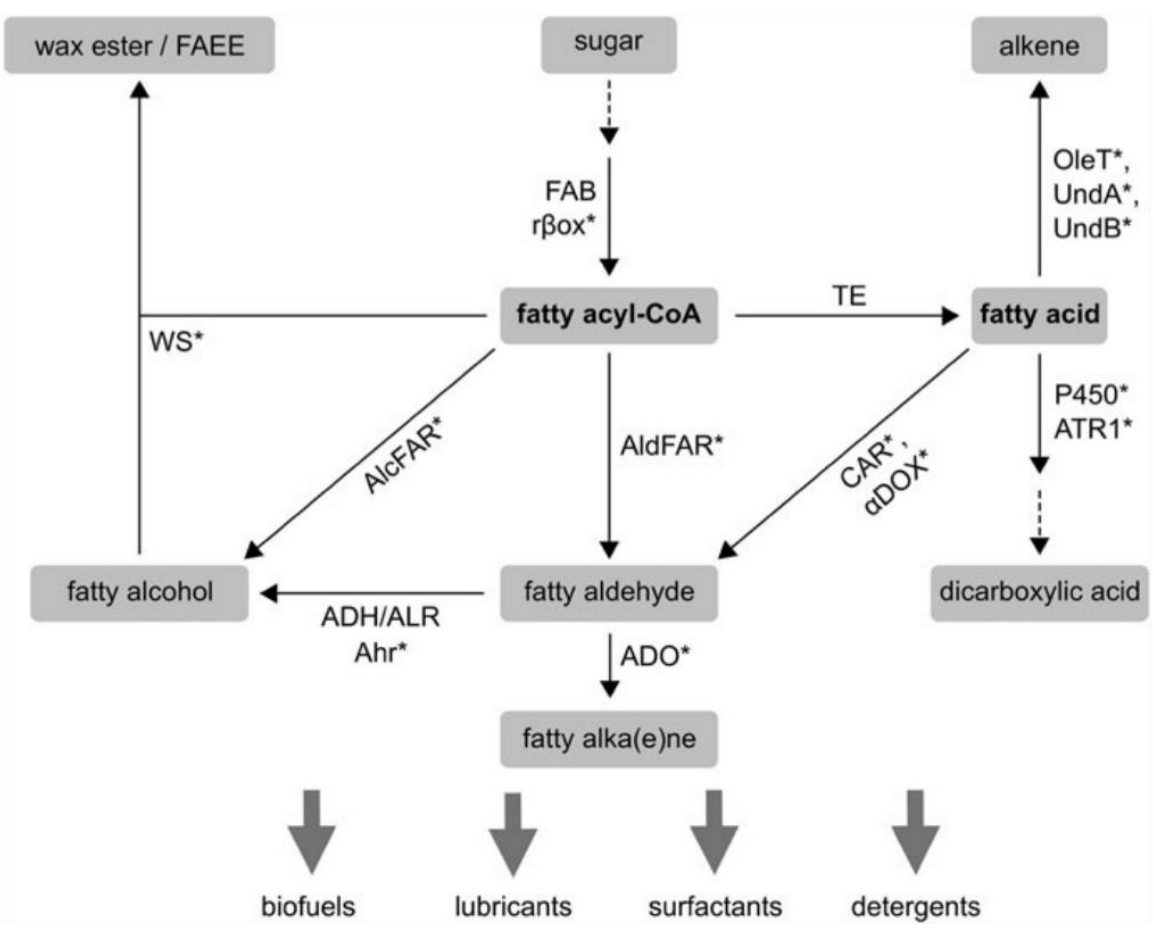

Fig. 3 Enzymatic routes for the production of FAderived compounds in S. cerevisiae. FA-derived compounds can be synthesized from the end product of FA biosynthesis (FAB) or reverse $\beta$-oxidation ( $\mathrm{r} \beta \mathrm{ox})$, the fatty acyl-CoA, and from free FA by several heterologous enzymes in yeast. FAs result from the cleavage of $\mathrm{CoA}$ by endogenous thioesterases (TE). Heterologous enzymes are marked with an asterisk. OleT $\mathrm{H}_{2} \mathrm{O}_{2}$ dependent P450 FA decarboxylase, UndA medium- chain FA-preferring nonheme iron oxidase, $U n d B$ membrane-bound desaturase-like enzyme, P450 cytochrome P450 CYP94C1, ATR1 cytochrome reductase from $A$. thaliana, CAR carboxylic acid reductase from M. marinum, $\alpha D O X \quad \alpha$-dioxygenase from $O$. sativa (rice), FAR fatty acyl-CoA reductase, ADO aldehyde deformylating oxygenase, $A D H$ alcohol dehydrogenase, $A L R$ aldehyde reductase, $A h r$ aldehyde reductase from E. coli, WS wax ester synthase 
chain in the end product is determined by the acyl chain of the FA precursor and by the specificity of the downstream enzymes.

\section{A. Alkenes}

Free FA can be converted to terminal alkenes by a one-step decarboxylation pathway, catalyzed by an $\mathrm{H}_{2} \mathrm{O}_{2}$-dependent cytochrome $\mathrm{P} 450$ enzyme, OleT (Chen et al. 2015; Zhou et al. 2018); a medium-chain FA-preferring nonheme iron oxidase, UndA (Zhu et al. 2017a; Zhou et al. 2018); or a membrane-bound desaturaselike enzyme, UndB (Zhou et al. 2018), together with a reduction system. The major challenge when applying these iron-dependent enzymes is the inefficient electron transfer, resulting in low titers in yeast due to inefficient cofactor or reducing systems (Zhou et al. 2018).

\section{B. Dicarboxylic Acids}

Utilizing the $\boldsymbol{\omega}$-oxidation pathway in S. cerevisiae enables the production of $\omega$-hydroxy- and $\alpha, \omega$-dicarboxylic acids ( $\omega$ meaning "last" $C$ atom when counted from the carboxyl group) from free FA. Such $\alpha$, $\omega$-dicarboxylic acids can serve as raw material for commodities and polymers (Han et al. 2017). Free FA can be terminally hydroxylated by a cytochrome P450 enzyme, followed by an oxidation to a carboxyl group by alcohol dehydrogenases and aldehyde dehydrogenases. Feeding of medium-chain FA to an $S$. cerevisiae strain expressing the cytochrome P450 enzyme CYP94C1, together with a cytochrome reductase, ATR1 from A. thaliana, allowed the production of $\omega$-hydroxy- and $\alpha, \omega$ dicarboxylic acids with chain lengths ranging from C10 to C16 (Han et al. 2017).

\section{Fatty Aldehydes, Alkanes, and Alcohols}

For the production of alkanes and alcohols, a fatty aldehyde intermediate is used as a precursor. There are several possible routes for the synthesis of fatty aldehydes. For instance, FA can be reduced through action of a carboxylic acid reductase (CAR) from Mycobacterium marinum (Zhou et al. 2016b; Tang et al. 2017; Henritzi et al. 2018), or it can be oxidatively decarboxylated by an $\alpha$-dioxygenase ( $\alpha$-DOX) from Oryza sativa (rice) (Jin et al. 2016; Foo et al. 2017). To be active, CAR requires a phosphopantetheinylation by a phosphopantetheinyl transferase (Akhtar et al. 2013). A third possibility is the reduction of CoA-bound FA to the corresponding aldehyde by fatty acylCoA reductases (AldFARs). Compared to CAR, it was shown that AldFAR-type enzymes are rather inefficient in yeast (Buijs et al. 2015; Zhou et al. 2016b). Aldehydes can be converted to odd-chain fatty alkanes $\left(\mathrm{C}_{n-1}\right)$ by cyanobacterial aldehyde deformylating oxygenases (ADO) or to fatty alcohols by endogenous alcohol dehydrogenases (ADHs) and aldehyde reductases (ALR) (Buijs et al. 2015; Zhou et al. 2016b; Kang et al. 2017; Zhu et al. 2017a). Zhou et al. (2016b) showed that the expression of a CAR together with its activating enzyme, phosphopantetheine transferase NpgA from $A$. nidulans, and an ADO, led to the synthesis of long-chain alkanes (Zhou et al. 2016b). To supply sufficient electrons, a reducing system was expressed additionally (Buijs et al. 2015). As previous studies showed that CAR also shows high activity toward medium-chain FA, Zhou et al. (2016a) produced medium-chain alkanes by screening different ADO orthologs and engineering their substrate binding sites (Zhu et al. 2017a). However, it is reported that ADO was not able to compete with the alcohol-forming enzymes (ADHs/ALRs) due to its low catalytic efficiency (Buijs et al. 2015; Zhou et al. 2016b; Foo et al. 2017; Kang et al. 2017; Zhu et al. 2017a). Another two-step pathway to produce alkanes was demonstrated by Foo et al. (2017). Expression of an $\alpha$-DOX from rice led to the production of long odd-chain fatty aldehyde intermediates, which can subsequently be deformylated to even-chain alkanes $\left(\mathrm{C}_{n-2}\right)$ by ADO (Foo et al. 2017). The advantage of a dioxygenase is that it uses dioxygen instead of NADPH for the production of aldehydes (Foo et al. 2017). Odd-chain fatty aldehydes produced by $\alpha$-DOX can also be oxidized to oddchain fatty alcohols through endogenous yeast ADHs/ALRs (Jin et al. 2016). Zhou et al. (2016b) 
explored ADHs/ALRs of yeasts and could show that Adh5 plays an important role in the production of long-chain alcohols, as overexpressing $A D H 5$ together with CAR increased the amount of long-chain alcohols (Zhou et al. 2016b). So far, the expression of fatty alcohol pathways resulted in the production of a pool of long- or medium-chain fatty alcohols with different chain lengths. Recently, our group was able to selectively produce the diesel-like alcohol 1-octanol in S. cerevisiae by combining a C8 FA-producing FAS with a two-step reduction pathway composed of CAR together with the phosphopantetheinyl transferase Sfp from Bacillus subtilis and the aldehyde reductase Ahr from E. coli (Henritzi et al. 2018). It has been shown that Ahr accepts a broad range of aliphatic aldehydes with chain lengths from $\mathrm{C} 4$ to C16 (Akhtar et al. 2013).

Another way to produce fatty alcohols is a one-step reduction of the fatty acyl-CoA, catalyzed by fatty acyl-CoA reductases (AlcFARs) (Runguphan and Keasling 2014). Zhou et al. (2016b) expressed the CAR/Adh5 pathway together with a fatty acyl-CoA reductase to produce fatty alcohols simultaneously from free FA and acyl-CoAs (Zhou et al. 2016b). Other labs have also reported the functional expression of several heterologous AlcFARs (Feng et al. 2015; d'Espaux et al. 2017). The advantage of onestep reactions, like the direct conversion from fatty acyl-CoAs to alcohols by AlcFARs or the FA decarboxylation pathway for alkene production, is the reduced intermediate metabolite loss and the circumvention of toxic intermediates (Chen et al. 2015). Besides medium-chain and long-chain FA-derived chemicals, very long-chain FA-derived products also play an important role as ingredients for lubricants, detergents, polymers, photographic filmprocessing agents, coatings, cosmetics, and pharmaceuticals (Yu et al. 2017). Yu et al. (2017) were able to selectively produce docosanol (C22) from very long-chain FA by rewiring the native FA elongation system and overexpressing a heterologous mycobacteria FAS I system, which provides high levels of C22 FA as direct precursor, together with a specific AlcFAR (Yu et al. 2017).

\section{Wax Esters and Fatty Acid Ethyl Esters}

Further compounds of interest include wax esters, which are typically esters of long-chain FA and long-chain alcohols and are used in personal care products, lubricants, or coatings (Wenning et al. 2017). They can be synthesized by wax ester synthases (WS) from alcohols and fatty acyl-CoA thioesters (Shi et al. 2012; Runguphan and Keasling 2014). Esters of ethanol and FA with chain lengths ranging from $\mathrm{C} 14$ to C20 (FAEE) represent suitable diesel fuels (Shi et al. 2012). Shi et al. (2012) could functionally express different WSs from different species and characterize their substrate preference. Unfortunately, some of the WSs from bacteria are bifunctional enzymes, which function as WS and acyl-CoA:diacylglycerol acyltransferase (DGAT), resulting in TAG formation, leading to a depletion of the acyl-CoA precursor pool (Shi et al. 2012). Eriksen et al. (2015) investigated the heterologous expression of a FAS I from Brevibacterium ammoniagenes coupled with a WS/DGAT to produce FAEEs. This strategy has the advantage of providing additional FA for growth supplementation and to supply the FA needed for FAEE synthesis (Eriksen et al. 2015). The microbial synthesis of wax esters by the esterification of a (very) long-chain fatty acylCoA with a primary, very long-chain fatty alcohol was reported by Wenning et al. (2017). The group combined the expression of a heterologous FAR with the expression of a plant WS to synthesize different jojoba-like wax esters. The used FAR reduced long-chain fatty acyl-CoAs, which resulted from de novo FA biosynthesis and elongation, to a long-chain alcohol. The plant-derived WS enabled the esterification of this alcohol with a long-chain fatty acyl-CoA in S. cerevisiae (Wenning et al. 2017).

Overall, there are still many obstacles that have to be overcome when using these heterologous enzymes, such as the challenging expression in the heterologous yeast host, low catalytic efficiency, inefficient electron transfer, or loss of intermediates by competing pathways. These issues have to be tackled in order to increase yields and titers of FA-derived compounds. One promising strategy could be a 
compartmentalization into peroxisomes by a peroxisomal targeting of pathway enzymes (Sheng et al. 2016; Zhou et al. 2016a). Similarly, blocking competing pathways through deletion of specific genes is a common strategy. For instance, the deletion of HFD1 was shown to be a crucial step for alkane and alcohol biosynthesis (Buijs et al. 2015; Zhou et al. 2016b). HFD1 encodes an aldehyde dehydrogenase involved in sphingolipid degradation and coenzyme Q biosynthesis and catalyzes the oxidation of fatty aldehydes to FA (Zhu et al. 2017a). Additionally, many engineering strategies for increasing the supply of the precursor AcCoA and free FA also led to an increase in the production of FA-derived products (Zhou et al. 2016b; Teixeira et al. 2017).

\section{Optimization of Fermentation Conditions}

Yeast FA titers keep rising through a plethora of metabolic engineering efforts; however, general production process optimization is just as important to unravel the full potential of producer strains. In oleaginous yeasts, like Y. lipolytica, lipid overproduction starts with the exhaustion of a primary nutrient, i.e., when entering stationary growth (Beopoulos et al. 2009). Consequently, nitrogen and/or glucose limitation has been utilized to increase lipid production in $S$. cerevisiae (Thompson and Trinh 2014; Yu et al. 2018). Such limiting conditions led to high lipid titers in a highly engineered S. cerevisiae strain, producing $33.4 \mathrm{~g} \mathrm{~L}^{-1}$ free FA in a fed-batch fermentation-the highest reported free FA titer by microbial fermentation to date (Yu et al. 2018). These results also emphasize the importance of the transition from flask cultivation to fed-batch cultivation in a fermenter. The latter permits a tight control of cultivation parameters, such as $\mathrm{pH}$, aeration, and nutrient supply, thereby enabling higher titers of FA and derivatives (Thompson and Trinh 2014; Zhou et al. 2016b; Yu et al. 2018).

In a very recent study, the effect of "forced" FA synthesis on the metabolism and physiology of an engineered $S$. cerevisiae strain was analyzed (Gossing et al. 2018). Such a systematic characterization could help to determine the crucial parameters for improving yeast lipid production. Besides the expected increase in $\beta$-oxidation and storage lipids, the analyzed strain also showed higher levels of oxidative stress and decreased amino acid levels (Gossing et al. 2018). Therefore, a viable approach could be an adjustment of media composition, e.g., by providing higher amounts of amino acids, or overexpressing genes to improve the amino acid uptake or synthesis.

Several previous studies have demonstrated a positive effect of amino acid supplementation on lipid accumulation. The addition of methionine led to increased palmitoleic acid production (Kamisaka et al. 2015), whereas high leucine levels led to increased lipid accumulation in a $\Delta s n f 2$ strain (Kamisaka et al. 2007). The additional supplementation of several other amino acids has been demonstrated to contribute to ethanol tolerance in yeast (Hirasawa et al. 2007; Sekine et al. 2007; Pham and Wright 2008; Yoshikawa et al. 2009). Nevertheless, it is also important to consider the genetic background of the FA producing strain at hand, as working with auxotrophic strains can decrease growth (Baganz et al. 1997; Çakar et al. 1999; Basso et al. 2010). Additionally, for some strain series, such as the BY strains, the importance of sufficient amino acid supply for optimal growth has been emphasized (Hanscho et al. 2012).

Vitamins, such as biotin and pantothenate, also play pivotal roles for normal yeast growth and are essential in FA synthesis (Suomalainen and Keränen 1963; Tehlivets et al. 2007). The addition of pantothenate, for example, was used as a metabolic switch to regulate the synthesis of $\beta$-farnesene, an AcCoA-derived sesquiterpene (Sandoval et al. 2014). This method could possibly be transferred to FA production processes, as they, similarly to $\beta$-farnesene, depend on CoA intermediates.

The fermentation temperature has a major influence not only on yeast growth but also on FA tolerance and production (Piper 1995; Viegas and Sá-Correia 1995; Viegas 1997). An engineered $S$. cerevisiae produced more palmitoleic acid at low temperatures $\left(20-25^{\circ} \mathrm{C}\right)$ (Kamisaka et al. 2015), and the oleaginous yeast Metschnikowia pulcherrima was shown to produce high lipid levels at low temperatures and $\mathrm{pH}$ 
(Santamauro et al. 2014). However, at lower temperatures, octanoic and decanoic acid are also more toxic (Viegas and Sá-Correia 1995; Viegas 1997). Furthermore, low temperatures might not be feasible in large-scale production, in which high gravity can lead to elevated temperatures, and cooling of big fermentation plants is cost-intensive (Gibson et al. 2007). Therefore, it might be more desirable to generate thermotolerant $S$. cerevisiae strains by introducing traits of thermotolerant yeasts, such as Kluyveromyces marxianus (Cernak et al. 2018), or even thermotolerant oleaginous yeasts, like $R$. toruloides ( $\mathrm{Wu}$ et al. 2018). Another important cultivation parameter for FA production is the $\mathrm{pH}$ and media buffering. Short- and medium-chain FAs are more toxic to $S$. cerevisiae at lower $\mathrm{pH}$ values, causing decreased viability and yields (Viegas et al. 1989). Therefore, it was shown that the addition of potassium phosphate buffer to complex medium elevated short- and medium-chain FA production substantially (Gajewski et al. 2017).

A promising approach to avoid the reuptake of the products by the cells and simultaneously decrease the effects of product toxicity is an in situ extraction. The addition of dodecane to the culture of a long-chain fatty alcoholproducing S. cerevisiae was successfully applied for the extraction of these compounds (Runguphan and Keasling 2014; d'Espaux et al. 2017). Upon addition of dodecane to a culture broth of a 1-octanol producing $S$. cerevisiae strain, however, production was reduced-likely due to the extraction of the precursor octanoic acid by dodecane (Henritzi et al. 2018). Therefore, when considering in situ extraction, an agent needs to be found, which is highly specific for each product, and does not remove important pathway intermediates from the culture.

Current efforts for yeast FA production were carried out in minimal (Zhou et al. 2016b; Yu et al. 2018) as well as complex medium (Leber et al. 2015; Gajewski et al. 2017). To be truly more sustainable than petroleum-based production, and to prevent a competition with food supply, starting materials, such as lignocellulosic biomass from agri- cultural waste, need to be utilized. To extract fermentable sugars from such biomass, it first must undergo pretreatment, in the process of which fermentation inhibitors arise. Therefore, the ideal FA producing yeast needs to be resistant to these inhibitors, as well as to be able to utilize glucose and five-carbon sugars, such as xylose, as carbon sources (Peralta-Yahya et al. 2012). D'Espaux et al. (2017) presented the first-and, so far, only-report about FA/alcohol production with $S$. cerevisiae solely from lignocellulosic feedstock. They fed the nonfood crops sorghum and switchgrass, which were pretreated with ionic liquids, to an engineered S. cerevisiae strain and obtained up to $0.7 \mathrm{~g} \mathrm{~L}^{-1}$ fatty alcohols (d'Espaux et al. 2017). By combining the different engineering and cultivation strategies mentioned above, a further increase in FA titers produced from lignocellulosic biomass can be foreseen.

\section{Conclusions}

Much progress has been made in recent years in the development of strategies for microbial production of FA and their derivatives from renewable feedstocks. The available literature shows that extensive interventions into the central carbon metabolism, relying on expression of heterologous pathways and manipulation of the activity of endogenous enzymes, are necessary to enable a high yield of oleochemicals in different chassis organisms. The highest yields of microbially produced oleochemicals reported to date were achieved in S. cerevisiae, owing to the great body of knowledge regarding the physiology of this yeast $(0.1 \mathrm{~g}$ free $\mathrm{FA} / \mathrm{g}$ glucose, i.e., approximately $30 \%$ of the theoretical yield). Moreover, FA production in S. cerevisiae has become more chain length-specific, and many FAs and a great variety of derivatives have already been produced successfully in labscale fermentations. The development of biosensors and high-throughput screening methods has become increasingly important and will considerably accelerate the development for well-performing strains. Once such challenges 
as metabolic imbalances and product toxicity have been tackled, yields, titers, and productivity could reach economically viable levels before long.

Acknowledgments L.B., F.W., and M.O. received funding from the European Union's Horizon 2020 research and innovation program under Grant Agreement No. 720824. S.B. received funding from the German Federal Ministry of Food and Agriculture following a decision of the German Bundestag under the Grant Number 22026315. The responsibility for the content of this publication lies with the authors. The authors thank Eckhard Boles for helpful comments on the manuscript.

\section{References}

Akhtar MK, Turner NJ, Jones PR (2013) Carboxylic acid reductase is a versatile enzyme for the conversion of fatty acids into fuels and chemical commodities. Proc Natl Acad Sci U S A 110:87-92

Alexandre H, Mathieu B, Charpentier C (1996) Alteration in membrane fluidity and lipid composition, and modulation of H+-ATPase activity in Saccharomyces cerevisiae caused by decanoic acid. Microbiology 142:469-475

Alper H, Fischer C, Nevoigt E, Stephanopoulos G (2005) Tuning genetic control through promoter engineering. Proc Natl Acad Sci U S A 102:1267812683

Alper H, Moxley J, Nevoigt E, Fink GR, Stephanopoulos G (2006) Engineering yeast transcription machinery for improved ethanol tolerance and production. Science 314:1565-1568

Baganz F, Hayes A, Marren D, Gardner DCJ, Oliver SG (1997) Suitability of replacement markers for functional analysis studies in Saccharomyces cerevisiae. Yeast 13:1563-1573

Basso TO, Dario MG, Tonso A, Stambuk BU, Gombert AK (2010) Insufficient uracil supply in fully aerobic chemostat cultures of Saccharomyces cerevisiae leads to respiro-fermentative metabolism and double nutrient-limitation. Biotechnol Lett 32:973-977

Baumann L, Rajkumar AS, Morrissey JP, Boles E, Oreb M (2018) A yeast-based biosensor for screening of short- and medium-chain fatty acid production. ACS Synth Biol 7:2640-2646

Becker S, Schmoldt H-U, Adams TM, Wilhelm S, Kolmar H (2004) Ultra-high-throughput screening based on cell-surface display and fluorescenceactivated cell sorting for the identification of novel biocatalysts. Curr Opin Biotechnol 15:323329

Beopoulos A, Chardot T, Nicaud J-M (2009) Yarrowia lipolytica: a model and a tool to understand the mechanisms implicated in lipid accumulation. Biochimie 91:692-696

Besada-Lombana PB, Fernandez-Moya R, Fenster J, Da Silva NA (2017) Engineering Saccharomyces cerevisiae fatty acid composition for increased tolerance to octanoic acid. Biotechnol Bioeng 114:1531-1538

Black PN, DiRusso CC (2007) Yeast acyl-CoA synthetases at the crossroads of fatty acid metabolism and regulation. Biochim Biophys Acta 1771:286298

Bocanegra JA, Scrutton NS, Perham RN (1993) Creation of an NADP-dependent pyruvate dehydrogenase multienzyme complex by protein engineering. Biochemistry 32:2737-2740

Brignole EJ, Smith S, Asturias FJ (2009) Conformational flexibility of metazoan fatty acid synthase enables catalysis. Nat Struct Mol Biol 16:190-197

Buijs NA, Zhou YJ, Siewers V, Nielsen J (2015) Longchain alkane production by the yeast Saccharomyces cerevisiae. Biotechnol Bioeng 112:1275-1279

Çakar ZP, Sauer U, Bailey JE (1999) Metabolic engineering of yeast: the perils of auxotrophic hosts. Biotechnol Lett 21:611-616

Cardenas J, Da Silva NA (2016) Engineering cofactor and transport mechanisms in Saccharomyces cerevisiae for enhanced acetyl-CoA and polyketide biosynthesis. Metab Eng 36:80-89

Caspeta L, Chen Y, Ghiaci P, Feizi A, Buskov S, Hallström BM, Petranovic D, Nielsen J (2014) Altered sterol composition renders yeast thermotolerant. Science 346:75-78

Cernak P, Estrela R, Poddar S, Skerker JM, Cheng Y-F, Carlson AK, Chen B, Glynn VM, Furlan M, Ryan OW, Donnelly MK, Arkin AP, Taylor JW, Cate JHD (2018) Engineering Kluyveromyces marxianus as a robust synthetic biology platform host. MBio 9:116

Chen B, Ling H, Chang MW (2013a) Transporter engineering for improved tolerance against alkane biofuels in Saccharomyces cerevisiae. Biotechnol Biofuels 6:21

Chen Y, Daviet L, Schalk M, Siewers V, Nielsen J (2013b) Establishing a platform cell factory through engineering of yeast acetyl-CoA metabolism. Metab Eng 15:48-54

Chen B, Lee D-Y, Chang MW (2015) Combinatorial metabolic engineering of Saccharomyces cerevisiae for terminal alkene production. Metab Eng 31:5361

Choi JW, Da Silva NA (2014) Improving polyketide and fatty acid synthesis by engineering of the yeast acetyl-CoA carboxylase. J Biotechnol 187:56-59

Clomburg JM, Vick JE, Blankschien MD, RodríguezMoyá M, Gonzalez R (2012) A synthetic biology approach to engineer a functional reversal of the $\beta$-oxidation cycle. ACS Synth Biol 1:541-554

Clomburg JM, Blankschien MD, Vick JE, Chou A, Kim $S$, Gonzalez R (2015) Integrated engineering of $\beta$ - 
oxidation reversal and $\omega$-oxidation pathways for the synthesis of medium chain $\omega$-functionalized carboxylic acids. Metab Eng 28:202-212

Cottrell M, Viljoen BC, Kock JLF, Lategan PM (1986) The long-chain fatty acid compositions of species representing the genera Saccharomyces, Schwanniomyces and Lipomyces. Microbiology 132:24012403

Curran KA, Karim AS, Gupta A, Alper HS (2013) Use of expression-enhancing terminators in Saccharomyces cerevisiae to increase mRNA half-life and improve gene expression control for metabolic engineering applications. Metab Eng 19:88-97

d'Espaux L, Mendez-Perez D, Li R, Keasling JD (2015) Synthetic biology for microbial production of lipid-based biofuels. Curr Opin Chem Biol 29:58-65

d'Espaux L, Ghosh A, Runguphan W, Wehrs M, Xu F, Konzock O, Dev I, Nhan M, Gin J, Reider Apel A, Petzold CJ, Singh S, Simmons BA, Mukhopadhyay A, García Martín H, Keasling JD (2017) Engineering high-level production of fatty alcohols by Saccharomyces cerevisiae from lignocellulosic feedstocks. Metab Eng 42:115-125

Da Silva NA, Srikrishnan S (2012) Introduction and expression of genes for metabolic engineering applications in Saccharomyces cerevisiae. FEMS Yeast Res 12:197-214

David F, Nielsen J, Siewers V (2016) Flux control at the malonyl-CoA node through hierarchical dynamic pathway regulation in Saccharomyces cerevisiae. ACS Synth Biol 5:224-233

Davis López SA, Griffith DA, Choi B, Cate JHD, Tullman-Ercek D (2018) Evolutionary engineering improves tolerance for medium-chain alcohols in Saccharomyces cerevisiae. Biotechnol Biofuels $11: 36$

de Jong BW, Shi S, Siewers V, Nielsen J (2014) Improved production of fatty acid ethyl esters in Saccharomyces cerevisiae through up-regulation of the ethanol degradation pathway and expression of the heterologous phosphoketolase pathway. Microb Cell Factories 13:39

Dekishima Y, Lan EI, Shen CR, Cho KM, Liao JC (2011) Extending carbon chain length of 1-butanol pathway for 1-hexanol synthesis from glucose by engineered Escherichia coli. J Am Chem Soc 133:11399-11401

Dellomonaco C, Clomburg JM, Miller EN, Gonzalez R (2011) Engineered reversal of the $\beta$-oxidation cycle for the synthesis of fuels and chemicals. Nature 476:355-359

Deparis Q, Claes A, Foulquié-Moreno MR, Thevelein JM (2017) Engineering tolerance to industrially relevant stress factors in yeast cell factories. FEMS Yeast Res 17:861

Dietrich JA, McKee AE, Keasling JD (2010) Highthroughput metabolic engineering: advances in small-molecule screening and selection. Annu Rev Biochem 79:563-590

Dragosits M, Mattanovich D (2013) Adaptive laboratory evolution-principles and applications for biotechnology. Microb Cell Factories 12:64

Eriksen DT, HamediRad M, Yuan Y, Zhao H (2015) Orthogonal fatty acid biosynthetic pathway improves fatty acid ethyl ester production in Saccharomyces cerevisiae. ACS Synth Biol 4:808-814

Feng X, Lian J, Zhao H (2015) Metabolic engineering of Saccharomyces cerevisiae to improve 1hexadecanol production. Metab Eng 27:10-19

Fernandez-Moya R, Da Silva NA (2017) Engineering Saccharomyces cerevisiae for high-level synthesis of fatty acids and derived products. FEMS Yeast Res 17:87

Fernandez-Moya R, Leber C, Cardenas J, Da Silva NA (2015) Functional replacement of the Saccharomyces cerevisiae fatty acid synthase with a bacterial type II system allows flexible product profiles. Biotechnol Bioeng 112:2618-2623

Foo JL, Susanto AV, Keasling JD, Leong SSJ, Chang MW (2017) Whole-cell biocatalytic and de novo production of alkanes from free fatty acids in Saccharomyces cerevisiae. Biotechnol Bioeng 114:232-237

Gajewski J, Pavlovic R, Fischer M, Boles E, Grininger M (2017) Engineering fungal de novo fatty acid synthesis for short chain fatty acid production. Nat Commun 8:14650

Gibson BR, Lawrence SJ, Leclaire JPR, Powell CD, Smart KA (2007) Yeast responses to stresses associated with industrial brewery handling. FEMS Microbiol Rev 31:535-569

González-Ramos D, van den Broek M, van Maris AJA, Pronk JT, Daran J-MG (2013) Genome-scale analyses of butanol tolerance in Saccharomyces cerevisiae reveal an essential role of protein degradation. Biotechnol Biofuels 6:48

González-Ramos D, Gorter de Vries AR, Grijseels SS, van Berkum MC, Swinnen S, van den Broek M, Nevoigt E, Daran J-MG, Pronk JT, van Maris AJA (2016) A new laboratory evolution approach to select for constitutive acetic acid tolerance in Saccharomyces cerevisiae and identification of causal mutations. Biotechnol Biofuels 9:19

Gossing M, Smialowska A, Nielsen J (2018) Impact of forced fatty acid synthesis on metabolism and physiology of Saccharomyces cerevisiae. FEMS Yeast Res 18:153

Han L, Peng Y, Zhang Y, Chen W, Lin Y, Wang Q (2017) Designing and creating a synthetic omega oxidation pathway in Saccharomyces cerevisiae enables production of medium-chain $\alpha$, $\omega$-dicarboxylic acids. Front Microbiol 8:1123

Hanscho M, Ruckerbauer DE, Chauhan N, Hofbauer HF, Krahulec S, Nidetzky B, Kohlwein SD, Zanghellini J, Natter K (2012) Nutritional requirements of the BY series of Saccharomyces 
cerevisiae strains for optimum growth. FEMS Yeast Res 12:796-808

Henritzi S, Fischer M, Grininger M, Oreb M, Boles E (2018) An engineered fatty acid synthase combined with a carboxylic acid reductase enables de novo production of 1-octanol in Saccharomyces cerevisiae. Biotechnol Biofuels 11:150

Hettema EH, Tabak HF (2000) Transport of fatty acids and metabolites across the peroxisomal membrane. Biochim Biophys Acta 1486:18-27

Hettema EH, van Roermund CW, Distel B, van den Berg M, Vilela C, Rodrigues-Pousada C, Wanders RJ, Tabak HF (1996) The ABC transporter proteins Pat1 and Pat2 are required for import of longchain fatty acids into peroxisomes of Saccharomyces cerevisiae. EMBO J 15:3813-3822

Hiltunen JK, Mursula AM, Rottensteiner H, Wierenga RK, Kastaniotis AJ, Gurvitz A (2003) The biochemistry of peroxisomal $\beta$-oxidation in the yeast Saccharomyces cerevisiae. FEMS Microbiol Rev 27:35-64

Hirasawa T, Yoshikawa K, Nakakura Y, Nagahisa K, Furusawa C, Katakura Y, Shimizu H, Shioya S (2007) Identification of target genes conferring ethanol stress tolerance to Saccharomyces cerevisiae based on DNA microarray data analysis. J Biotechnol 131:34-44

Hofbauer HF, Schopf FH, Schleifer H, Knittelfelder OL, Pieber B, Rechberger GN, Wolinski H, Gaspar ML, Kappe CO, Stadlmann J, Mechtler K, Zenz A, Lohner K, Tehlivets O, Henry SA, Kohlwein SD (2014) Regulation of gene expression through a transcriptional repressor that senses acyl-chain length in membrane phospholipids. Dev Cell 29:729-739

Hu Y, Zhu Z, Nielsen J, Siewers V (2018) Heterologous transporter expression for improved fatty alcohol secretion in yeast. Metab Eng 45:51-58

Jin Z, Wong A, Foo JL, Ng J, Cao Y-X, Chang MW, Yuan Y-J (2016) Engineering Saccharomyces cerevisiae to produce odd chain-length fatty alcohols. Biotechnol Bioeng 113:842-851

Johnson DR, Knoll LJ, Rowleyo N, Gordon JI (1994) Genetic analysis of the role of Saccharomyces cerevisiae acyl-CoA synthetase genes in regulating protein N-myristoylation. J Biol Chem 269 (27):18037-18046. http://www.jbc.org/content/ 269/27/18037.full.pdf

Johnson AO, Gonzalez-Villanueva M, Wong L, Steinbüchel A, Tee KL, Xu P, Wong TS (2017) Design and application of genetically-encoded malonylCoA biosensors for metabolic engineering of microbial cell factories. Metab Eng 44:253-264

Kamisaka Y, Tomita N, Kimura K, Kainou K, Uemura H (2007) DGA1 (diacylglycerol acyltransferase gene) overexpression and leucine biosynthesis significantly increase lipid accumulation in the $\Delta s n f 2$ disruptant of Saccharomyces cerevisiae. Biochem J 408:61-68
Kamisaka Y, Kimura K, Uemura H, Yamaoka M (2015) Addition of methionine and low cultivation temperatures increase palmitoleic acid production by engineered Saccharomyces cerevisiae. Appl Microbiol Biotechnol 99:201-210

Kaneda K, Imaizumi S, Yano I (1995) Distribution of C22-, C24- and C26- $\alpha$-unit-containing mycolic acid homologues in mycobacteria. Microbiol Immunol 39:563-570

Kang M-K, Zhou YJ, Buijs NA, Nielsen J (2017) Functional screening of aldehyde decarbonylases for long-chain alkane production by Saccharomyces cerevisiae. Microb Cell Factories 16:320

Kim S, Gonzalez R (2018) Selective production of decanoic acid from iterative reversal of $\beta$-oxidation pathway. Biotechnol Bioeng 115:1311-1320

Kim S, Clomburg JM, Gonzalez R (2015) Synthesis of medium-chain length (C6-C10) fuels and chemicals via $\beta$-oxidation reversal in Escherichia coli. J Ind Microbiol Biotechnol 42:465-475

Knappe J, Schacht J, Möckel W, Höpner T, Vetter H, Edenharder R (1969) Pyruvate formate-lyase reaction in Escherichia coli. The enzymatic system converting an inactive form of the lyase into the catalytically active enzyme. Eur J Biochem 11:316327

Knoll LJ, Johnson DR, Gordon JI (1994) Biochemical studies of three Saccharomyces cerevisiae acyl-CoA synthetases, Faalp, FaaZp, and Faa3p. J Biol Chem 269:16348-16356

Kocharin K, Chen Y, Siewers V, Nielsen J (2012) Engineering of acetyl-CoA metabolism for the improved production of polyhydroxybutyrate in Saccharomyces cerevisiae. AMB Express 2:52

Kocharin K, Siewers V, Nielsen J (2013) Improved polyhydroxybutyrate production by Saccharomyces cerevisiae through the use of the phosphoketolase pathway. Biotechnol Bioeng 110:2216-2224

Koopman F, Beekwilder J, Crimi B, van Houwelingen A, Hall RD, Bosch D, van Maris AJA, Pronk JT, Daran J-M (2012) De novo production of the flavonoid naringenin in engineered Saccharomyces cerevisiae. Microb Cell Factories 11:155

Kozak BU, van Rossum HM, Luttik MAH, Akeroyd M, Benjamin KR, Wu L, de Vries S, Daran J-M, Pronk JT, van Maris AJA (2014a) Engineering acetyl coenzyme A supply: functional expression of a bacterial pyruvate dehydrogenase complex in the cytosol of Saccharomyces cerevisiae. MBio 5: e01696-e01614

Kozak BU, van Rossum HM, Benjamin KR, Wu L, Daran J-MG, Pronk JT, van Maris AJA (2014b) Replacement of the Saccharomyces cerevisiae acetyl-CoA synthetases by alternative pathways for cytosolic acetyl-CoA synthesis. Metab Eng 21:46-59

Kozak BU, van Rossum HM, Niemeijer MS, van Dijk M, Benjamin K, Wu L, Daran J-MG, Pronk JT, van Maris AJA (2016) Replacement of the initial steps 
of ethanol metabolism in Saccharomyces cerevisiae by ATP-independent acetylating acetaldehyde dehydrogenase. FEMS Yeast Res 16:fow006

Krivoruchko A, Serrano-Amatriain C, Chen Y, Siewers V, Nielsen J (2013) Improving biobutanol production in engineered Saccharomyces cerevisiae by manipulation of acetyl-CoA metabolism. J Ind Microbiol Biotechnol 40:1051-1056

Krivoruchko A, Zhang Y, Siewers V, Chen Y, Nielsen J (2015) Microbial acetyl-CoA metabolism and metabolic engineering. Metab Eng 28:28-42

Kruis AJ, Gallone B, Jonker T, Mars AE, van Rijswijck IMH, JCM W-R, Smid EJ, Steensels J, Verstrepen KJ, SWM K, van der Oost J, Weusthuis RA (2018) Contribution of Eat1 and other alcohol acyltransferases to ester production in Saccharomyces cerevisiae. Front Microbiol 9:345

Lafon-Lafourcade S, Geneix C, Ribéreau-Gayon P (1984) Inhibition of alcoholic fermentation of grape must by fatty acids produced by yeasts and their elimination by yeast ghosts. Appl Environ Microbiol 47:1246-1249

Leber C, Da Silva NA (2014) Engineering of Saccharomyces cerevisiae for the synthesis of short chain fatty acids. Biotechnol Bioeng 111:347-358

Leber C, Polson B, Fernandez-Moya R, Da Silva NA (2015) Overproduction and secretion of free fatty acids through disrupted neutral lipid recycle in Saccharomyces cerevisiae. Metab Eng 28:54-62

Leber C, Choi JW, Polson B, Da Silva NA (2016) Disrupted short chain specific $\beta$-oxidation and improved synthase expression increase synthesis of short chain fatty acids in Saccharomyces cerevisiae. Biotechnol Bioeng 113:895-900

Lee ME, DeLoache WC, Cervantes B, Dueber JE (2015) A highly characterized yeast toolkit for modular, multipart assembly. ACS Synth Biol 4:975-986

Legras JL, Erny C, Le Jeune C, Lollier M, Adolphe Y, Demuyter C, Delobel P, Blondin B, Karst F (2010) Activation of two different resistance mechanisms in Saccharomyces cerevisiae upon exposure to octanoic and decanoic acids. Appl Environ Microbiol 76:7526-7535

Leibundgut M, Maier T, Jenni S, Ban N (2008) The multienzyme architecture of eukaryotic fatty acid synthases. Curr Opin Struct Biol 18:714-725

Lennen RM, Pfleger BF (2013) Microbial production of fatty acid-derived fuels and chemicals. Curr Opin Biotechnol 24:1044-1053

Li X, Guo D, Cheng Y, Zhu F, Deng Z, Liu T (2014) Overproduction of fatty acids in engineered Saccharomyces cerevisiae. Biotechnol Bioeng 111:1841-1852

Li S, Si T, Wang M, Zhao H (2015) Development of a synthetic malonyl-CoA sensor in Saccharomyces cerevisiae for intracellular metabolite monitoring and genetic screening. ACS Synth Biol 4:1308-1315
Lian J, Zhao H (2015) Reversal of the $\beta$-oxidation cycle in Saccharomyces cerevisiae for production of fuels and chemicals. ACS Synth Biol 4:332-341

Lian J, Si T, Nair NU, Zhao H (2014) Design and construction of acetyl-CoA overproducing Saccharomyces cerevisiae strains. Metab Eng 24:139-149

Ling H, Chen B, Kang A, Lee J-M, Chang M (2013) Transcriptome response to alkane biofuels in Saccharomyces cerevisiae: identification of efflux pumps involved in alkane tolerance. Biotechnol Biofuels 6:95

Liu H, Liu K, Yan M, Xu L, Ouyang P (2011) gTME for improved adaptation of Saccharomyces cerevisiae to corn cob acid hydrolysate. Appl Biochem Biotechnol 164:1150-1159

Liu P, Chernyshov A, Najdi T, Fu Y, Dickerson J, Sandmeyer S, Jarboe L (2013) Membrane stress caused by octanoic acid in Saccharomyces cerevisiae. Appl Microbiol Biotechnol 97:3239-3251

Liu W, Zhang B, Jiang R (2017) Improving acetyl-CoA biosynthesis in Saccharomyces cerevisiae via the overexpression of pantothenate kinase and PDH bypass. Biotechnol Biofuels 10:97

Lomakin IB, Xiong Y, Steitz TA (2007) The crystal structure of yeast fatty acid synthase, a cellular machine with eight active sites working together. Cell 129:319-332

Mans R, Daran J-MG, Pronk JT (2018) Under pressure: evolutionary engineering of yeast strains for improved performance in fuels and chemicals production. Curr Opin Biotechnol 50:47-56

Marella ER, Holkenbrink C, Siewers V, Borodina I (2018) Engineering microbial fatty acid metabolism for biofuels and biochemicals. Curr Opin Biotechnol 50:39-46

Meadows AL, Hawkins KM, Tsegaye Y, Antipov E, Kim Y, Raetz L, Dahl RH, Tai A, Mahatdejkul-Meadows T, Xu L, Zhao L, Dasika MS, Murarka A, Lenihan J, Eng D, Leng JS, Liu C-L, Wenger JW, Jiang H, Chao L, Westfall P, Lai J, Ganesan S, Jackson P, Mans R, Platt D, Reeves CD, Saija PR, Wichmann G, Holmes VF, Benjamin K, Hill PW, Gardner TS, Tsong AE (2016) Rewriting yeast central carbon metabolism for industrial isoprenoid production. Nature 537:694-697

Membrillo-Hernandez J, Echave P, Cabiscol E, Tamarit J, Ros J, Lin EC (2000) Evolution of the adhE gene product of Escherichia coli from a functional reductase to a dehydrogenase. Genetic and biochemical studies of the mutant proteins. J Biol Chem 275:33869-33875

Michener JK, Thodey K, Liang JC, Smolke CD (2012) Applications of genetically-encoded biosensors for the construction and control of biosynthetic pathways. Metab Eng 14:212-222

Mukherjee K, Bhattacharyya S, Peralta-Yahya P (2015) GPCR-based chemical biosensors for mediumchain fatty acids. ACS Synth Biol 4:1261-1269 
Nielsen J, Larsson C, van Maris A, Pronk J (2013) Metabolic engineering of yeast for production of fuels and chemicals. Curr Opin Biotechnol 24:398404

Nygård Y, Mojzita D, Toivari M, Penttilä M, Wiebe MG, Ruohonen L (2014) The diverse role of Pdr12 in resistance to weak organic acids. Yeast 31:219-232

Oelkers P, Tinkelenberg A, Erdeniz N, Cromley D, Billheimer JT, Sturley SL (2000) A lecithin cholesterol acyltransferase-like gene mediates diacylglycerol esterification in yeast. J Biol Chem 275:1560915612

Oelkers P, Cromley D, Padamsee M, Billheimer JT, Sturley SL (2002) The DGA1 gene determines a second triglyceride synthetic pathway in yeast. J Biol Chem 277:8877-8881

Oh CS, Toke DA, Mandala S, Martin CE (1997) ELO2 and ELO3, homologues of the Saccharomyces cerevisiae ELO1 gene, function in fatty acid elongation and are required for sphingolipid formation. J Biol Chem 272:17376-17384

Paddon CJ, Westfall PJ, Pitera DJ, Benjamin K, Fisher K, McPhee D, Leavell MD, Tai A, Main A, Eng D, Polichuk DR, Teoh KH, Reed DW, Treynor T, Lenihan J, Jiang H, Fleck M, Bajad S, Dang G, Dengrove D, Diola D, Dorin G, Ellens KW, Fickes S, Galazzo J, Gaucher SP, Geistlinger T, Henry R, Hepp M, Horning T, Iqbal T, Kizer L, Lieu B, Melis D, Moss N, Regentin R, Secrest S, Tsuruta H, Vazquez R, Westblade LF, Xu L, Yu M, Zhang Y, Zhao L, Lievense J, Covello PS, Keasling JD, Reiling KK, Renninger NS, Newman JD (2013) Highlevel semi-synthetic production of the potent antimalarial artemisinin. Nature 496:528-532

Peralta-Yahya PP, Zhang F, del Cardayre SB, Keasling JD (2012) Microbial engineering for the production of advanced biofuels. Nature 488:320-328

Pfleger BF, Gossing M, Nielsen J (2015) Metabolic engineering strategies for microbial synthesis of oleochemicals. Metab Eng 29:1-11

Pham TK, Wright PC (2008) The proteomic response of Saccharomyces cerevisiae in very high glucose conditions with amino acid supplementation. J Proteome Res 7:4766-4774

Piper PW (1995) The heat shock and ethanol stress responses of yeast exhibit extensive similarity and functional overlap. FEMS Microbiol Lett 134:121-127

Pronk JT, Yde Steensma H, van Dijken JP (1996) Pyruvate metabolism in Saccharomyces cerevisiae. Yeast 12:1607-1633

Rigouin C, Gueroult M, Croux C, Dubois G, Borsenberger V, Barbe S, Marty A, Daboussi F, André I, Bordes F (2017) Production of medium chain Fatty acids by Yarrowia lipolytica: combining molecular design and TALEN to engineer the fatty acid synthase. ACS Synth Biol 6:1870-1879

Rodriguez S, Denby CM, van Vu T, Baidoo EEK, Wang G, Keasling JD (2016) ATP citrate lyase mediated cytosolic acetyl-CoA biosynthesis increases mevalonate production in Saccharomyces cerevisiae. Microb Cell Factories 15:169

Royce LA, Yoon JM, Chen Y, Rickenbach E, Shanks JV, Jarboe LR (2015) Evolution for exogenous octanoic acid tolerance improves carboxylic acid production and membrane integrity. Metab Eng 29:180-188

Runguphan W, Keasling JD (2014) Metabolic engineering of Saccharomyces cerevisiae for production of fatty acid-derived biofuels and chemicals. Metab Eng 21:103-113

Rupilius W, Ahma S (2006) The changing world of oleochemicals. Palm Oil Dev 44:21-28

Sandoval CM, Ayson M, Moss N, Lieu B, Jackson P, Gaucher SP, Horning T, Dahl RH, Denery JR, Abbott DA, Meadows AL (2014) Use of pantothenate as a metabolic switch increases the genetic stability of farnesene producing Saccharomyces cerevisiae. Metab Eng 25:215-226

Santamauro F, Whiffin FM, Scott RJ, Chuck CJ (2014) Low-cost lipid production by an oleaginous yeast cultured in non-sterile conditions using model waste resources. Biotechnol Biofuels 7:34

Sanz P (2003) Snf1 protein kinase: a key player in the response to cellular stress in yeast. Biochem Soc Trans 31:178-181

Sarria S, Kruyer NS, Peralta-Yahya P (2017) Microbial synthesis of medium-chain chemicals from renewables. Nat Biotechnol 35:1158-1166

Schadeweg V, Boles E (2016a) Increasing n-butanol production with Saccharomyces cerevisiae by optimizing acetyl-CoA synthesis, NADH levels and trans-2-enoyl-CoA reductase expression. Biotechnol Biofuels 9:1

Schadeweg V, Boles E (2016b) n-Butanol production in Saccharomyces cerevisiae is limited by the availability of coenzyme A and cytosolic acetyl-CoA. Biotechnol Biofuels 9:1

Schallmey M, Frunzke J, Eggeling L (2014) Looking for the pick of the bunch: high-throughput screening of producing microorganisms with biosensors. Curr Opin Biotechnol 26:148-154

Scharnewski M, Pongdontri P, Mora G, Hoppert M, Fulda M (2008) Mutants of Saccharomyces cerevisiae deficient in acyl-CoA synthetases secrete fatty acids due to interrupted fatty acid recycling. FEBS J 275:2765-2778

Schmidt JH (2015) Life cycle assessment of five vegetable oils. J Clean Prod 87:130-138

Schweizer E, Hofmann J (2004) Microbial type I fatty acid synthases (FAS): major players in a network of cellular FAS systems. Microbiol Mol Biol Rev 68:501-517. Table of contents

Sekine T, Kawaguchi A, Hamano Y, Takagi H (2007) Desensitization of feedback inhibition of the Saccharomyces cerevisiae $\gamma$-glutamyl kinase enhances proline accumulation and freezing tolerance. Appl Environ Microbiol 73:4011-4019 
Sheng J, Stevens J, Feng X (2016) Pathway compartmentalization in peroxisome of Saccharomyces cerevisiae to produce versatile medium chain fatty alcohols. Sci Rep 6:177

Shi S, Valle-Rodríguez JO, Khoomrung S, Siewers V, Nielsen J (2012) Functional expression and characterization of five wax ester synthases in Saccharomyces cerevisiae and their utility for biodiesel production. Biotechnol Biofuels 5:7

Shi S, Valle-Rodríguez JO, Siewers V, Nielsen J (2014a) Engineering of chromosomal wax ester synthase integrated Saccharomyces cerevisiae mutants for improved biosynthesis of fatty acid ethyl esters. Biotechnol Bioeng 111:1740-1747

Shi S, Chen Y, Siewers V, Nielsen J (2014b) Improving production of malonyl coenzyme A-derived metabolites by abolishing Snf1-dependent regulation of Accl. MBio 5:321

Shi S, Ang EL, Zhao H (2018) In vivo biosensors: mechanisms, development, and applications. J Ind Microbiol Biotechnol 45:491-516

Shiba Y, Paradise EM, Kirby J, Ro D-K, Keasling JD (2007) Engineering of the pyruvate dehydrogenase bypass in Saccharomyces cerevisiae for high-level production of isoprenoids. Metab Eng 9:160-168

Shin G-H, Veen M, Stahl U, Lang C (2012) Overexpression of genes of the fatty acid biosynthetic pathway leads to accumulation of sterols in Saccharomyces cerevisiae. Yeast 29:371-383

Skjoedt ML, Snoek T, Kildegaard KR, Arsovska D, Eichenberger M, Goedecke TJ, Rajkumar AS, Zhang J, Kristensen M, Lehka BJ, Siedler S, Borodina I, Jensen MK, Keasling JD (2016) Engineering prokaryotic transcriptional activators as metabolite biosensors in yeast. Nat Chem Biol 12:951-958

Smidt O, Du Preez JC, Albertyn J (2012) Molecular and physiological aspects of alcohol dehydrogenases in the ethanol metabolism of Saccharomyces cerevisiae. FEMS Yeast Res 12:33-47

Stincone A, Prigione A, Cramer T, Wamelink MMC, Campbell K, Cheung E, Olin-Sandoval V, Grüning N-M, Krüger A, Tauqeer Alam M, Keller MA, Breitenbach M, Brindle KM, Rabinowitz JD, Ralser M (2015) The return of metabolism: biochemistry and physiology of the pentose phosphate pathway. Biol Rev Camb Philos Soc 90:927-963

Sumper M, Oesterhelt D, Riepertinger C, Lynen F (1969) Die Synthese verschiedener Carbonsäuren durch den Multienzymkomplex der Fettsäuresynthese aus Hefe und die Erklärung ihrer Bildung. Eur J Biochem 10:377-387

Suomalainen H, Keränen AJA (1963) The effect of biotin deficiency on the synthesis of fatty acids by yeast. Biochim Biophys Acta 70:493-503

Tang X, Chen WN (2015) Enhanced production of fatty alcohols by engineering the TAGs synthesis pathway in Saccharomyces cerevisiae. Biotechnol Bioeng 112:386-392
Tang X, Feng H, Chen WN (2013) Metabolic engineering for enhanced fatty acids synthesis in Saccharomyces cerevisiae. Metab Eng 16:95-102

Tang X, Feng L, Chen L, Chen WN (2017) Engineering Saccharomyces cerevisiae for efficient biosynthesis of fatty alcohols based on enhanced supply of free fatty acids. ACS Omega 2:3284-3290

Tehlivets O, Scheuringer K, Kohlwein SD (2007) Fatty acid synthesis and elongation in yeast. Biochim Biophys Acta 1771:255-270

Teixeira PG, Ferreira R, Zhou YJ, Siewers V, Nielsen J (2017) Dynamic regulation of fatty acid pools for improved production of fatty alcohols in Saccharomyces cerevisiae. Microb Cell Factories 16:1185

Teo WS, Hee KS, Chang MW (2013) Bacterial FadR and synthetic promoters function as modular fatty acid sensor- regulators in Saccharomyces cerevisiae. Eng Life Sci 13:456-463

Thompson RA, Trinh CT (2014) Enhancing fatty acid ethyl ester production in Saccharomyces cerevisiae through metabolic engineering and medium optimization. Biotechnol Bioeng 111:2200-2208

Toke DA, Martin CE (1996) Isolation and characterization of a gene affecting fatty acid elongation in Saccharomyces cerevisiae. J Biol Chem 271:1841318422

Valle-Rodríguez JO, Shi S, Siewers V, Nielsen J (2014) Metabolic engineering of Saccharomyces cerevisiae for production of fatty acid ethyl esters, an advanced biofuel, by eliminating non-essential fatty acid utilization pathways. Appl Energy 115:226-232

van Roermund CW, Tabak HF, van den Berg M, Wanders RJ, Hettema EH (2000) Pex11p plays a primary role in medium-chain fatty acid oxidation, a process that affects peroxisome number and size in Saccharomyces cerevisiae. J Cell Biol 150:489498

van Roermund CW, Drissen R, van den Berg M, Ijlst L, Hettema EH, Tabak HF, Waterham HR, Wanders RJ (2001) Identification of a peroxisomal ATP carrier required for medium-chain fatty acid $\beta$-oxidation and normal peroxisome proliferation in Saccharomyces cerevisiae. Mol Cell Biol 21:43214329

van Roermund CWT, Waterham HR, Ijlst L, Wanders RJA (2003) Fatty acid metabolism in Saccharomyces cerevisiae. Cell Mol Life Sci 60:1838-1851

van Rossum HM, Kozak BU, Niemeijer MS, Duine HJ, Luttik MAH, Boer VM, Kötter P, Daran J-MG, van Maris AJA, Pronk JT (2016a) Alternative reactions at the interface of glycolysis and citric acid cycle in Saccharomyces cerevisiae. FEMS Yeast Res 16: fow017

van Rossum HM, Kozak BU, Pronk JT, van Maris AJA (2016b) Engineering cytosolic acetyl-coenzyme A supply in Saccharomyces cerevisiae: pathway stoichiometry, free-energy conservation and redoxcofactor balancing. Metab Eng 36:99-115 
van Rossum HM, Kozak BU, Niemeijer MS, Dykstra JC, Luttik MAH, Daran J-MG, van Maris AJA, Pronk JT (2016c) Requirements for carnitine shuttlemediated translocation of mitochondrial acetyl moieties to the yeast cytosol. MBio 7:517

Viegas C (1997) Effects of low temperatures $\left(9-33{ }^{\circ} \mathrm{C}\right)$ and $\mathrm{pH}(3.3-5.7)$ in the loss of Saccharomyces cerevisiae viability by combining lethal concentrations of ethanol with octanoic and decanoic acids. Int J Food Microbiol 34:267-277

Viegas CA, Sá-Correia I (1995) Toxicity of octanoic acid in Saccharomyces cerevisiae at temperatures between 8.5 and $30^{\circ} \mathrm{C}$. Enzym Microb Technol $17: 826-831$

Viegas CA, Rosa MF, Sá-Correia I, Novais JM (1989) Inhibition of yeast growth by octanoic and decanoic acids produced during ethanolic fermentation. Appl Environ Microbiol 55:21-28

Viegas CA, Almeida PF, Cavaco M, Sá-Correia I (1998) The $\mathrm{H}(+)$-ATPase in the plasma membrane of Saccharomyces cerevisiae is activated during growth latency in octanoic acid-supplemented medium accompanying the decrease in intracellular $\mathrm{pH}$ and cell viability. Appl Environ Microbiol 64:779-783

Vorapreeda T, Thammarongtham C, Cheevadhanarak S, Laoteng K (2012) Alternative routes of acetylCoA synthesis identified by comparative genomic analysis: involvement in the lipid production of oleaginous yeast and fungi. Microbiology (Reading, England) 158:217-228

Waks Z, Silver PA (2009) Engineering a synthetic dualorganism system for hydrogen production. Appl Environ Microbiol 75:1867-1875

Wang BL, Ghaderi A, Zhou H, Agresti J, Weitz DA, Fink GR, Stephanopoulos G (2014a) Microfluidic highthroughput culturing of single cells for selection based on extracellular metabolite production or consumption. Nat Biotechnol 32:473-478

Wang Y, Chen H, Yu O (2014b) A plant malonyl-CoA synthetase enhances lipid content and polyketide yield in yeast cells. Appl Microbiol Biotechnol 98:5435-5447

Wang M, Li S, Zhao H (2016) Design and engineering of intracellular-metabolite-sensing/regulation gene circuits in Saccharomyces cerevisiae. Biotechnol Bioeng 113:206-215

Wattanachaisaereekul S, Lantz AE, Nielsen ML, Nielsen J (2008) Production of the polyketide 6-MSA in yeast engineered for increased malonyl-CoA supply. Metab Eng 10:246-254

Welch JW, Burlingame AL (1973) Very long-chain fatty acids in yeast. J Bacteriol 115:464-466

Wenning L, Yu T, David F, Nielsen J, Siewers V (2017) Establishing very long-chain fatty alcohol and wax ester biosynthesis in Saccharomyces cerevisiae. Biotechnol Bioeng 114:1025-1035

Williams TC, Xu X, Ostrowski M, Pretorius IS, Paulsen IT (2017) Positive-feedback, ratiometric biosensor expression improves high-throughput metaboliteproducer screening efficiency in yeast. Synth Biol 2:1-13

Wu C-C, Tsai Y-Y, Ohashi T, Misaki R, Limtong S, Fujiyama K (2018) Isolation of a thermotolerant Rhodosporidium toruloides DMKU3-TK16 mutant and its fatty acid profile at high temperature. FEMS Microbiol Lett 365(21). https://doi.org/ 10.1093/femsle/fny203

Xu P, Qiao K, Ahn WS, Stephanopoulos G (2016) Engineering Yarrowia lipolytica as a platform for synthesis of drop-in transportation fuels and oleochemicals. Proc Natl Acad Sci U S A 113:10848-10853

Xue S-J, Chi Z, Zhang Y, Li Y-F, Liu G-L, Jiang H, Hu Z, Chi Z-M (2018) Fatty acids from oleaginous yeasts and yeast-like fungi and their potential applications. Crit Rev Biotechnol 38:1049-1060

Yang H, Bard M, Bruner DA, Gleeson A, Deckelbaum RJ, Aljinovic G, Pohl TM, Rothstein R, Sturley SL (1996) Sterol esterification in yeast: a two-gene process. Science (New York, NY) 272:1353-1356

Yoshikawa K, Tanaka T, Furusawa C, Nagahisa K, Hirasawa T, Shimizu H (2009) Comprehensive phenotypic analysis for identification of genes affecting growth under ethanol stress in Saccharomyces cerevisiae. FEMS Yeast Res 9:32-44

Yu A-Q, Pratomo Juwono NK, Leong SSJ, Chang MW (2014) Production of fatty acid-derived valuable chemicals in synthetic microbes. Front Bioeng Biotechnol 2:1219

Yu A-Q, Pratomo Juwono NK, Foo JL, Leong SSJ, Chang MW (2016) Metabolic engineering of Saccharomyces cerevisiae for the overproduction of short branched-chain fatty acids. Metab Eng 34:36-43

Yu T, Zhou YJ, Wenning L, Liu Q, Krivoruchko A, Siewers V, Nielsen J, David F (2017) Metabolic engineering of Saccharomyces cerevisiae for production of very long chain fatty acid-derived chemicals. Nat Commun 8:15587

Yu T, Zhou YJ, Huang M, Liu Q, Pereira R, David F, Nielsen J (2018) Reprogramming yeast metabolism from alcoholic fermentation to lipogenesis. Cell 174:1549-1558.e14

Zhang F, Carothers JM, Keasling JD (2012) Design of a dynamic sensor-regulator system for production of chemicals and fuels derived from fatty acids. Nat Biotechnol 30:354-359

Zhang Y, Nielsen J, Liu Z (2018) Metabolic engineering of Saccharomyces cerevisiae for production of fatty acid-derived hydrocarbons. Biotechnol Bioeng 110:87

Zhou YJ, Buijs NA, Zhu Z, Gómez DO, Boonsombuti A, Siewers V, Nielsen J (2016a) Harnessing yeast peroxisomes for biosynthesis of fatty-acid-derived biofuels and chemicals with relieved side-pathway competition. J Am Chem Soc 138:15368-15377

Zhou YJ, Buijs NA, Zhu Z, Qin J, Siewers V, Nielsen J (2016b) Production of fatty acid-derived oleo- 
chemicals and biofuels by synthetic yeast cell factories. Nat Commun 7:34

Zhou YJ, Hu Y, Zhu Z, Siewers V, Nielsen J (2018) Engineering 1-alkene biosynthesis and secretion by dynamic regulation in yeast. ACS Synth Biol 7:584-590

Zhu Z, Zhou YJ, Kang M-K, Krivoruchko A, Buijs NA, Nielsen J (2017a) Enabling the synthesis of medium chain alkanes and 1-alkenes in yeast. Metab Eng 44:81-88
Zhu Z, Zhou YJ, Krivoruchko A, Grininger M, Zhao ZK, Nielsen J (2017b) Expanding the product portfolio of fungal type I fatty acid synthases. Nat Chem Biol 13:360-362

Zou Z, DiRusso CC, Ctrnacta V, Black PN (2002) Fatty acid transport in Saccharomyces cerevisiae. Directed mutagenesis of FAT1 distinguishes the biochemical activities associated with Fatlp. J Biol Chem 277:31062-31071 


\subsection{Transcriptomic response of Saccharomyces cerevisiae to octanoic acid production}

Declaration of author contributions to the publication:

Transcriptomic response of Saccharomyces cerevisiae to octanoic acid production

Status: accepted, February 2021

Journal: FEMS Yeast Research

Type of manuscript: research article

Contributing authors: Leonie Baumann (LB), Tyler Doughty (TD), Verena Siewers

(VS), Jens Nielsen (JN), Eckhard Boles (EB) \& Mislav Oreb (MO)

Contributions of doctoral candidate and co-authors

(1) Concept and design

Doctoral candidate LB: $65 \%$

Co-authors TD, VS, JN, EB, MO: 10\%, 5\%, 5\%, 5\%, 10\%

(2) Conducting tests and experiments

Doctoral candidate LB: $90 \%$, yeast molecular biology, fermentations, metabolite extraction and GC analysis, toxicity test

Co-author TD: $10 \%$, RNA extraction

(3) Compilation of data sets and figures

Doctoral candidate LB: $100 \%$, RNA-Seq data, metabolite titers and growth, toxicity test

(4) Analysis and interpretation of data

Doctoral candidate LB: 70\%, RNA-Seq data, differential expression analysis, metabolite titers and growth, toxicity test

Co-authors TD, EB, MO: 10\%, 10\%, 10\%, RNA-Seq data, supervision and advice

(5) Drafting of manuscript

Doctoral candidate LB: $80 \%$

Co-authors TD, EB, MO: $10 \%, 5 \%, 5 \%$ 
Transcriptomic response of Saccharomyces cerevisiae to octanoic acid production

\author{
Leonie Baumann, ${ }^{1}$ Tyler Doughty, ${ }^{2}$ Verena Siewers, ${ }^{2,3}$ Jens Nielsen, ${ }^{2,3,4}$ Eckhard Boles ${ }^{1}$ and \\ Mislav Oreb*1 \\ ${ }^{1}$ Institute of Molecular Biosciences, Faculty of Biological Sciences, Goethe University Frankfurt, \\ Max-von-Laue Straße 9, 60438 Frankfurt am Main, Germany \\ ${ }^{2}$ Department of Biology and Biological Engineering, Chalmers University of Technology, SE- \\ 41296 Gothenburg, Sweden
}

${ }^{3}$ Novo Nordisk Foundation Center for Biosustainability, Chalmers University of Technology, SE41296 Gothenburg, Sweden

${ }^{4}$ Biolnnovation Institute, Ole Maaløes Vej 3, DK-2200 Copenhagen N, Denmark

*Corresponding author: m.oreb@bio.uni-frankfurt.de

\begin{abstract}
The medium-chain fatty acid octanoic acid is an important platform compound widely used in industry. The microbial production from sugars in Saccharomyces cerevisiae is a promising alternative to current non-sustainable production methods, however, titers need to be further increased. To achieve this, it is essential to have in-depth knowledge about the cell's physiology during octanoic acid production. To this end, we collected the first RNA-Seq data of an octanoic acid producer strain at three time points during fermentation. The strain produced higher levels of octanoic acid and increased levels of fatty acids of other chain lengths (C6-C18) but showed decreased growth compared to the reference. Furthermore, we show that the here analyzed transcriptomic response to internally produced octanoic acid is notably distinct from a wild type's response to externally supplied octanoic acid as reported in previous publications. By comparing the transcriptomic response of different sampling times, we identified several genes that we subsequently overexpressed and knocked out, respectively. Hereby we identified RPL4OB, to date unknown to play a role in fatty acid biosynthesis or medium-chain fatty acid tolerance. Overexpression of $R P L 4 O B$ led to an increase in octanoic acid titers by $40 \%$.
\end{abstract}

KEYWORDS: Octanoic acid, transcriptome response to medium-chain fatty acids, RNA-Seq, RPL4OB 


\section{INTRODUCTION}

Medium-chain fatty acids like octanoic acid (C8 fatty acid) have a wide range of applications in antimicrobials, surfactants and cosmetics, and can also serve as precursors for biofuels. ${ }^{1-4}$ At present, octanoic acid and its derivatives are produced from oil seed crops or crude oil. ${ }^{5}$ In comparison to petrochemical industry, the extraction from oily plants, such as oil palm, might appear environmentally less harmful but there are rising concerns about its extensive cultivation. Oil palm cultivation is often associated with deforestation of rainforest and the ecological consequences thereof, and in addition competes with food production. ${ }^{6}$ The engineering of microbes for octanoic acid production from renewable biomass is therefore a promising alternative. ${ }^{5,7}$

Yeast produces fatty acids in the fatty acid synthesis cycle through action of the cytosolic fatty acid synthase (FAS), a large enzymatic complex encoded by the two genes FAS1 and FAS2. Naturally, Saccharomyces cerevisiae produces mostly saturated and monounsaturated long-chain fatty acids with chain lengths of $\mathrm{C} 16$ and $\mathrm{C} 18 .{ }^{8}$ There are different ways to redirect production from longchain to short- and medium-chain fatty acids. Here, we use a yeast strain that carries a modified variant of $F A S 1$, namely $F A S^{R K}{ }^{9}$ This enzyme contains an amino acid exchange from arginine to lysine ("R1834K") in the malonyl-palmitoyl transferase (MPT) domain of Fas1p. Thereby, the loading of the precursor malonyl-CoA is reduced, leading to premature release of short acyl chains, such as octanoyl-CoA. Octanoyl-CoA is then hydrolysed by thioesterases and free octanoic acid is released. ${ }^{9}$ For an economically feasible production, further strain improvements and process optimizations are necessary. ${ }^{10}$

To increase titers, it is essential to advance the understanding of the producer strain's physiology in the course of octanoic acid production. ${ }^{11}$ To our knowledge, such an analysis has not been undertaken yet. So far, two transcriptome-wide studies have been performed with extracellular addition of octanoic acid to wild type strains..$^{12,13}$ In the first one, a microarray analysis showed that incubation with $0.05 \mathrm{mM}(7 \mathrm{mg} / \mathrm{L})$ octanoic acid for 20 minutes caused oxidative stress and a similar response to decanoic acid was observed. Overall, 75 genes were found to be differentially regulated in comparison to the non-exposed control. Pdr12 was identified as the main involved transporter and the transporter Tpo1 was shown to play a minor role in octanoic acid expulsion. ${ }^{12}$

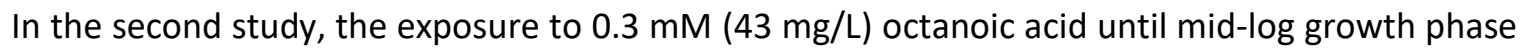
revealed differential expression of at least 2 -fold of 136 genes in comparison to the non-exposed control. Here, membrane leakiness was identified as a possible mechanism of cell disruption and increasing the oleic acid content was shown to enhance yeast tolerance to octanoic acid. ${ }^{13}$ 
To get a comprehensive insight into expression changes during production of octanoic acid, we performed RNA-Seq in three different growth phases of an S. cerevisiae octanoic acid producer strain and a reference strain. We used expression profiles from the different growth phases to guide strain engineering efforts and achieved a $40 \%$ increase in octanoic acid production.

\section{RESULTS}

Fermentation profiles of an octanoic acid producer compared to reference. To get the first insight into transcriptional changes in different growth phases associated with octanoic acid production, we performed RNA-Seq transcriptome profiling. As a background strain we used SHY34, ${ }^{14}$ a triple knockout strain ( $\triangle$ fas $1 \Delta$ fas $2 \triangle$ faa2). In this strain the two $F A S$ genes are knocked out as well as FAA2, encoding a fatty acyl-CoA synthetase, deletion of which prevents degradation of short- and medium-chain fatty acids. ${ }^{3,15}$ We transformed this strain either with a reference plasmid containing FAS1 and FAS2 in their wild type form fused into one sequence, i. e. fusFAS ${ }^{w t}$, or with a plasmid carrying $F A S 1^{R K}$ and $F A S 2$ fused into one sequence, namely fusFAS ${ }^{R K}$. The fusFAS ${ }^{R K}$ sequence contains an amino acid exchange from arginine to lysine in the MPT domain (R1834K), generating an octanoic acid producer. ${ }^{14}$

The two strains grew with a similar growth rate in exponential phase and only after the diauxic shift, growth was slower in the octanoic acid producer strain compared to the reference strain leading to a lower final OD in the octanoic acid producer (Figure 1A). Such impairment of growth has similarly been observed for non-producers spiked with octanoic acid. ${ }^{3}$ Samples were taken in early exponential growth phase (14 h, T1), shortly after the diauxic shift (22 h, T2) and in ethanol consumption phase (46 h, T3), respectively. To confirm octanoic acid production, samples were taken from the culture supernatants at each sampling time for fatty acid extraction and quantification via GC measurement (Figure 1B). In the reference strain, octanoic acid was produced in negligible amounts $(<7 \mathrm{mg} / \mathrm{L})$ at all three sampling times whereas in the octanoic acid producer, the amount increased gradually from $7 \mathrm{mg} / \mathrm{L}$ (T1) to $43 \mathrm{mg} / \mathrm{L}$ (T2) to $87 \mathrm{mg} / \mathrm{L}$ (T3). We also observed an enhanced production of other short- and medium-chain fatty acids, namely C6, C10 and C12 fatty acids, in the octanoic acid producer strain, with the most pronounced difference to the reference strain at T3 (Figure S1). Interestingly, even secreted amounts of saturated longchain fatty acids, i.e. C16:0 and C18:0 fatty acids, were higher in the octanoic acid producer compared to the reference strain at T2 and T3 (Figure 1C). 


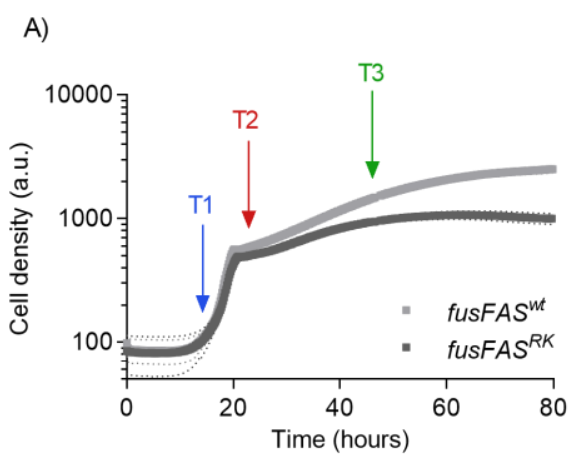

B)

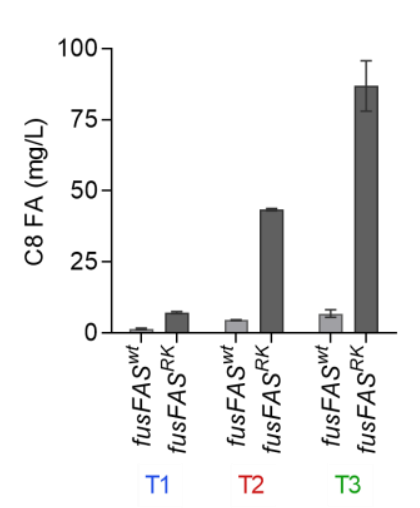

C)

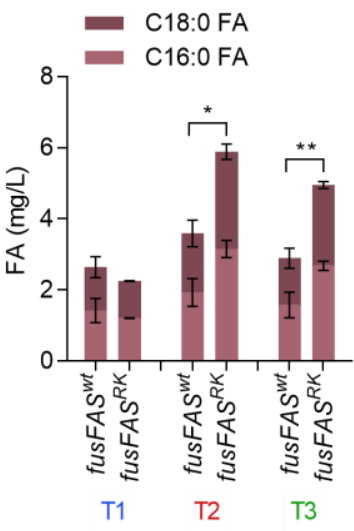

Figure 1. Characterization of growth and octanoic acid production. (A) Growth was monitored on a cell growth quantifier in triplicates for strains SHY34+fusFAS ${ }^{w t}$ (reference strain) and SHY34+fusFAS ${ }^{R K}$ (octanoic acid producer). Sampling times are indicated with arrows (T1 = 14 h; T2 = 22 h; T3 = 46 h). (B, C) C8, C16:0 and C18:0 fatty acids (FA) were extracted and quantified via GC. $n=3$, error bars $= \pm$ standard deviation. Statistical analysis was performed using two-tailed t test $\left({ }^{*} p<0.05,{ }^{* *} p<0.01\right)$.

Analysis of transcriptomic response in different phases of octanoic acid production. From the samples described above, we extracted RNA and performed RNA-Seq analysis. A principal component analysis (PCA) of the RNA-Seq data set revealed a close clustering of the three replicates at each sampling time in both strains (Figure S2).The data of the reference strain and the octanoic acid producer strain also clustered closely together, suggesting a limited number of differentially expressed genes (Figure S3). At T1, only 15 genes were upregulated $\left(\log _{2} F C>1\right.$, FDR $<0.01$ ) in the octanoic acid producer in comparison to the reference strain - at $\mathrm{T} 2$ this was the case for 29 genes and at T3 for 85 genes. The number of downregulated genes was in a similar range, with 11 genes downregulated at $\mathrm{T} 1,44$ genes at $\mathrm{T} 2$ and 144 genes at $\mathrm{T} 3$. The number of differentially expressed genes between the two strains therefore increased with fermentation time. A list of all differentially expressed genes can be found in a Supplementary File 1. GO term enrichment analyses for all three sampling times are attached as Supplementary File 2.

To get a comprehensive view of the transcriptomic landscape of the octanoic acid producer in different growth phases, we compared the RNA-Seq data of the three sampling times (Figure 2). We observed only eight genes upregulated and eleven genes downregulated at more than one sampling time compared to the reference. For upregulation, these included $F A S 1^{R K}, F A S 2$ as well as HIS3 - the latter being the selection marker of the fUSFAS ${ }^{R K}$ plasmid used in the experiment. A higher copy number or expression of fusFAS ${ }^{R K}$ was recently shown to increase octanoic acid titers. ${ }^{14}$ It is likely that the expression was upregulated in the octanoic acid producer to ensure that enough long-chain fatty acids are produced for cell growth since chain length control in 
fusFAS ${ }^{R K}$ is leaky. ${ }^{9}$ YOR2O3W was found to be upregulated at all sampling times, however, according to the SGD database, it is unlikely to encode a functional protein, and its assigned sequence partially overlaps with HIS3, suggesting an incorrect assignment in the RNA-Seq read mapping. HXT2, a glucose transporter, was the only gene found to be downregulated at all three sampling times.

(A) Upregulated

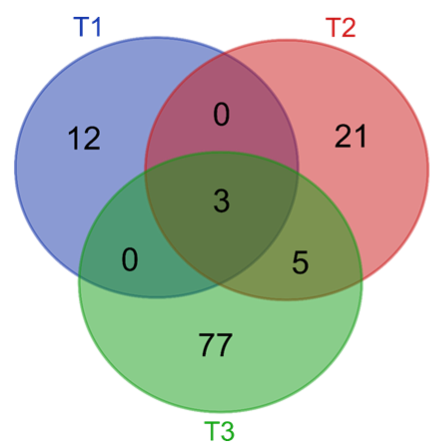

\begin{tabular}{|l|l|}
\hline Sampling time & Gene \\
\hline \multirow{3}{*}{$\mathrm{T} 1, \mathrm{~T} 2, \mathrm{~T} 3$} & YOR203W \\
\cline { 2 - 2 } & HIS3 \\
\cline { 2 - 2 } & FAS2 \\
\hline \multirow{5}{*}{$\mathrm{T} 2, \mathrm{~T} 3$} & YOR186W \\
\cline { 2 - 2 } & MCH2 \\
\cline { 2 - 2 } & IMD2 \\
\hline & EEB1 \\
\cline { 2 - 2 } & FAS1 \\
\hline
\end{tabular}

(B) Downregulated

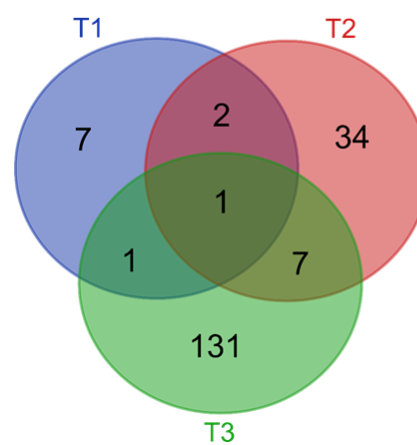

\begin{tabular}{|l|l|}
\hline Sampling time & Gene \\
\hline $\mathrm{T} 1, \mathrm{~T} 2, \mathrm{~T} 3$ & HXT2 \\
\hline $\mathrm{T} 1, \mathrm{~T} 2$ & MNC1 \\
\cline { 2 - 2 } & PTR2 \\
\hline $\mathrm{T} 1, \mathrm{~T} 3$ & BTN2 \\
\hline & URA7 \\
\cline { 2 - 2 } & NOP56 \\
\cline { 2 - 2 } T2, T3 & UTR2 \\
\cline { 2 - 2 } & YDR133C \\
\cline { 2 - 2 } & DAN1 \\
\hline & IZH4 \\
\hline & INA1 \\
\hline
\end{tabular}

Figure 2. Differential gene expression for the octanoic acid producer compared to reference. Number of genes that are upregulated $(A)$ or downregulated $(B)$ in the octanoic acid producer strain in comparison to the reference strain $\left(\log _{2} \mathrm{FC}>1, \mathrm{FDR}<0.01\right)$ between sampling times $(\mathrm{T} 1=14 \mathrm{~h} ; \mathrm{T} 2=22 \mathrm{~h} ; \mathrm{T} 3=46 \mathrm{~h})$. Gene lists include genes that are differentially regulated at more than one sampling time as indicated.

Comparison to published transcriptome data. Two other transcriptome-wide studies have been performed so far, however, both of them with extracellular addition of octanoic acid to wild type S. cerevisiae strains. ${ }^{12,13}$ In the first study, a microarray analysis by Legras and colleagues, ${ }^{12} 75$ genes were found to be differentially regulated $\left(\log _{2} F C \geq 0.3, F D R<0.05\right)$ in comparison to the non-exposed control after incubation with only $0.05 \mathrm{mM}(7 \mathrm{mg} / \mathrm{L})$ octanoic acid for the short period of 20 minutes. ${ }^{12}$ In the second study by Liu and colleagues, ${ }^{13}$ the exposure to six times the amount, i. e. $0.3 \mathrm{mM}(43 \mathrm{mg} / \mathrm{L})$ octanoic acid, led to differential expression $\left(\log _{2} \mathrm{FC}>1, p<0.01\right)$ in 136 genes in comparison to the non-exposed control. ${ }^{13}$ Interestingly, the overlap between these two published studies and our analysis was limited to a very small number of genes (Figures S4, S5). In all three studies the transporter Pdr12 was found to be upregulated (RNA-Seq: T3), which 
has previously been associated with octanoic acid expulsion. ${ }^{12}$ However, additional plasmid-based expression of Pdr12 has not led to an increase in octanoic acid titers in our hands, suggesting that endogenous expression is sufficient (Figure S6).

To understand expression changes in the octanoic acid producer, we wanted to further analyse the effect of differentially expressed genes. To limit the number of genes for further analysis, we decided to focus on those genes that were differentially expressed at more than one sampling time in our data. We reasoned that such genes might have a more profound effect on octanoic acid production and constitute more promising targets for engineering than genes that are differentially regulated at only one sampling time.

Overexpression of identified genes with a multi-copy plasmid library. We decided to test the effect of plasmid-based overexpression of all genes that were found to be up- or downregulated, respectively, in at least two sampling times in our data (see gene tables in Figure 2). In addition, we also tested the effect of overexpression of some genes overlapping with data from Liu and colleagues. ${ }^{13}$ For overexpressing the respective genes, we used a plasmid-library ${ }^{16}$ that contains the majority of the yeast genome cloned as approximately $10 \mathrm{~kb}$ fragments into multi-copy plasmid backbones. Two of the genes (DAN1, NOP56) were not represented in the library and were therefore cloned separately on a multi-copy plasmid backbone. To avoid plasmid burden effects, we tested the plasmids in a strain that contains $F A S 1^{R K}$ and FAS2 in their wild type loci (LBY38). Towards the end of the exponential growth phase (18 h) we evaluated the octanoic acid titers for some of the strains and observed a variation of titers in accordance with differing growth (Figure 3). LBY38 containing plasmids with IMD2, HXT2 or BTN2, respectively, had $\mathrm{OD}_{600}$ values and titers of only about $50 \%$ of the control, indicating delayed growth and therefore octanoic acid production.

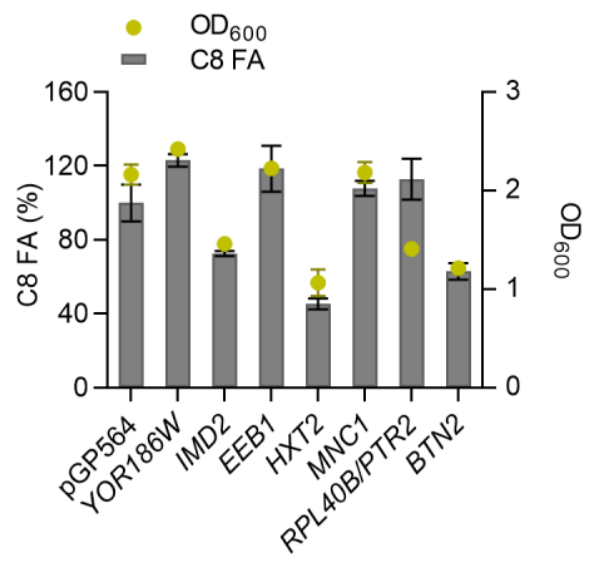

Figure 3. Fermentation of octanoic acid producer with overexpressed genes. The octanoic acid producer strain LBY38 was transformed with plasmids of a multi-copy library or a control plasmid (pGP564) and cultured for $18 \mathrm{~h}$ in buffered $\mathrm{SCD}^{\text {-leu }}$ medium. Tested library plasmids contained the genes indicated on the $x$-axis. Octanoic acid was extracted and quantified by GC measurement, and is shown in percent of the control (pGP564). $n=2$, error bars $= \pm$ standard deviation. 
In ethanol consumption phase ( $46 \mathrm{~h}$ ), all strains had reached similar $\mathrm{OD}_{600}$ values, which facilitated comparison between octanoic acid titers (Figure 4A). After $46 \mathrm{~h}$ of fermentation, the expression of the library plasmid containing two neighbouring genes of interest, RPL4OB and PTR2, yielded a $40 \%$ higher octanoic acid titer; whereas HXT2, BTN2 and IMD2 expression led to a $25 \%, 15 \%$ and $13 \%$ decrease, respectively. The expression of the library plasmid containing ECl1 led to a decrease in titer to about $60 \%$ of the reference. All other plasmids did not $(<10 \%$ difference) influence octanoic acid titers. To further characterize the effects on octanoic acid production, we generated individual knockout strains for HXT2, BTN2, IMD2 and ECI1 in the LBY38 background. We analysed octanoic acid titers after $46 \mathrm{~h}$ of growth in buffered SCD medium. All four knockout strains, $\Delta h x t 2, \Delta b t n 2, \Delta i m d 2$ and $\Delta e c i 1$, showed similar growth as well as octanoic acid titers as the control LBY38 (Figure 4B).

A)

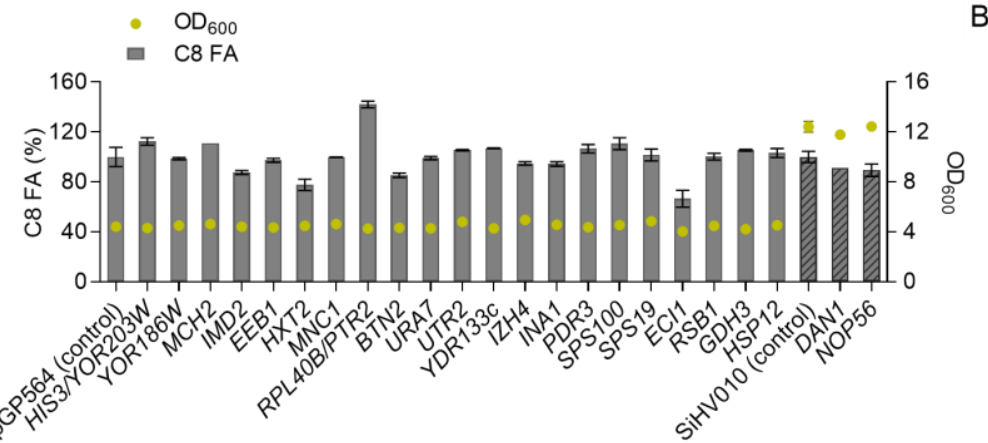

B)

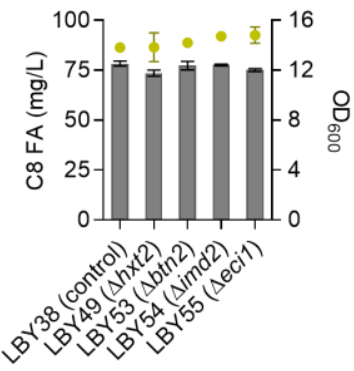

Figure 4. Test of overexpression and deletion of targets in octanoic acid producer. (A) The octanoic acid producer strain LBY38 was transformed with plasmids of a multi-copy library and cultured in buffered SCDleu medium. Tested library plasmids contained the genes indicated on the $\mathrm{x}$-axis (dark grey bars). The genes DAN1 and NOP56 were not part of the library and therefore were cloned individually into SiHV010 multicopy backbone, transformed into LBY38 and cultured in buffered YPD ${ }^{\text {hyg }}$ (striped bars). Titers are shown in percent of the respective control strains. (B) Four genes were knocked out individually in LBY38 and the strains were fermented in buffered YPD. For all samples, octanoic acid was extracted after $46 \mathrm{~h}$ of growth and quantified by GC measurement. $n=2$, error bars $= \pm$ standard deviation.

Overexpression of $R P L 4 O B$ leads to increase in octanoic acid production. To determine which of the two genes - RPLAOB or PTR2 - is responsible for the observed positive effect, we cloned each with its native promotor and terminator on a separate plasmid backbone. The fermentation showed that the plasmid-based expression of $R P L A O B$ caused an increase in octanoic acid titers of $40 \%$, whereas PTR2 expression did not influence octanoic acid titers (Figure 5A). The amount of secreted saturated C18 fatty acid was lower in the strain with plasmid-based RPL4OB expression compared to the reference. When introducing a second copy of RPLAOB into the genome under the control of the strong PPGK1 promoter, we could not observe this increase in titers (Figure 5B), suggesting that the native $R P L A O B$ regulatory elements are required for the positive effect on 
octanoic acid production. To investigate the effect of $R P L 4 O B$ on robustness towards octanoic acid, we transformed the non-producer CEN.PK2-1C with either a control plasmid or the multi-copy plasmid containing RPL4OB. After incubation for $20 \mathrm{~h}$ in a $96-$ well plate (without shaking) with 0 $300 \mathrm{mg} / \mathrm{L}$ octanoic acid, the strain with plasmid-based expression of RPL4OB showed strongly decreased growth under all octanoic acid concentrations and even without addition of octanoic acid (Figure 5C).
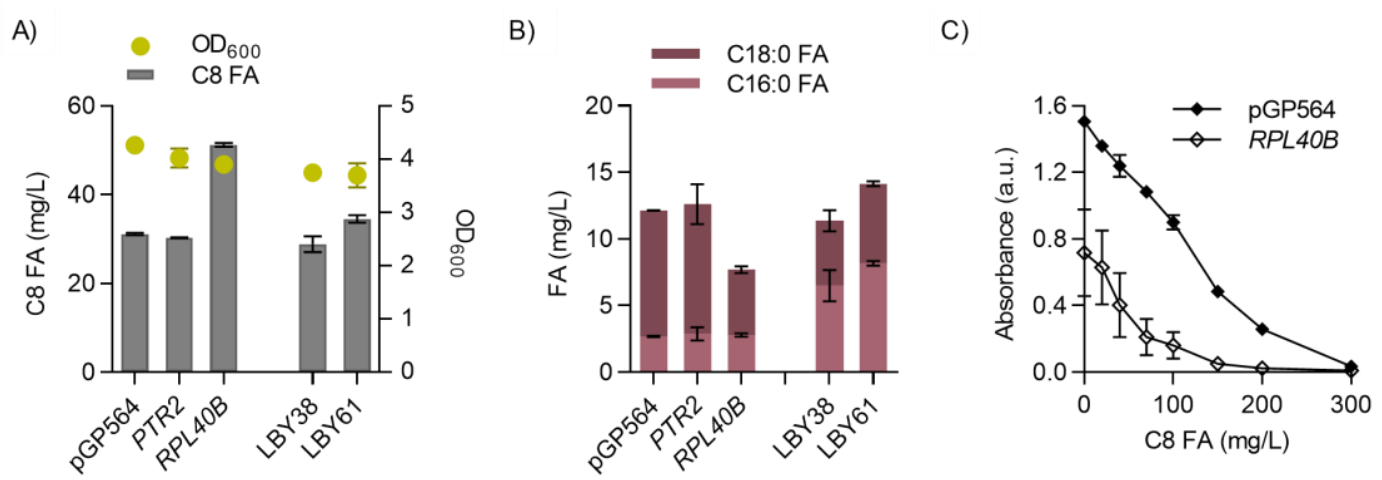

Figure 5. Effect of PTR2 and RPL4OB overexpression. (A, B) The octanoic acid producer strain LBY38 was transformed with plasmids containing PTR2, RPL4OB or the control vector (pGP564). A second copy of $R P L 4 O B$ was integrated into the genome under control of $p P G K 1$ in the PYK2 locus in LBY38 resulting in strain LBY61. Strains were cultured in buffered SCD medium and fatty acids (FA) were extracted after $46 \mathrm{~h}$ and quantified by GC measurement. $n=2$, error bars $= \pm$ standard deviation. (C) Non-producer CEN.PK2-1C containing either the vector pGP564 (control) or $R P L 4 O B$ were inoculated in triplicates to $\mathrm{OD}_{600}=0.05$ and supplied with 0-300 mg/L octanoic acid. Growth was measured by absorbance after $20 \mathrm{~h} . n=3$, error bars $= \pm$ standard deviation.

\section{DISCUSSION}

To date, no transcriptomics data of an S. cerevisiae octanoic acid producer strain was available. To fill this gap, we generated a high-quality RNA-Seq data set for three sampling times during fermentation. By comparing the growth, fatty acid and expression profile of the producer to a reference strain, we were able to get a profound insight into transcriptomic changes during octanoic acid production.

In the course of fermentation, octanoic acid biosynthesis increased in the producer strain from 7 $\mathrm{mg} / \mathrm{L}$ after $14 \mathrm{~h}$ to $87 \mathrm{mg} / \mathrm{L}$ after $46 \mathrm{~h}$. This increase correlated with the increase in differential expression in comparison to the reference strain, suggesting that increasing octanoic acid titers influence overall strain physiology. In terms of growth, the two strains behaved similarly until the diauxic shift when growth of the octanoic acid producer slowed down more quickly and reached lower final OD values. The main production of octanoic acid started shortly before or during the 
diauxic shift, which is consistent with the production profile observed in our previous studies., ${ }^{9,14}$ Although we did not measure the concentrations of glucose and ethanol in the course of fermentations shown in Figure 1, the sampling points can be clearly assigned to the glucose (T1) and ethanol consumption phases $(T 2, T 3)$, respectively, based on the previously published correlation of precisely measured growth curves and carbon source profiles. ${ }^{17}$ This implies that octanoic acid production mainly occurs during the consumption of ethanol, presumably due to an increased supply of the precursor molecule acetyl-CoA on this carbon source. The onset of octanoic acid production parallels the slowdown in growth; this can probably be attributed to toxic effects of octanoic acid on the cell physiology, ${ }^{3,12,13,18}$ which is further potentiated in the presence of ethanol. ${ }^{18,19}$ Octanoic acid disrupts the composition of the yeast plasma membrane leading to increased leakage and at high concentrations cell death. ${ }^{12,13}$ This effect was shown to be mitigated by an increase in the average chain length of membrane fatty acids, i.e. more C18 than C16 fatty acids, as well as an increase in unsaturated fatty acid, i.e. oleic acid (C18:1). ${ }^{13,20}$ In the culture supernatant, unsaturated long-chain fatty acids were not detectable. Interestingly, at T2 and T3, we observed that the octanoic acid producer secreted more saturated long-chain fatty acids of chain lengths C16 and C18 than the reference strain. Since the strain used in the experiment is a $\Delta f a s 1 \Delta f a s 2 \Delta f a a 2$ deletion strain, its only possibility to produce long-chain fatty acids is the leaky synthesis through plasmid-encoded fusFAS ${ }^{R K}$. Even though the "R1834K" amino acid exchange in the MPT domain of FAS1 leads to premature release of short- and medium-chain fatty acids from the FAS cycle, this regulation is not completely tight. ${ }^{9}$ As the pressure to synthesize enough longchain fatty acids, which are essential for growth and survival, is probably high, the copy number of the plasmid containing fusFAS ${ }^{R K}$ seems to have been increased. The evidence for this is twofold: Firstly, we saw higher expression of the fusFAS ${ }^{R K}$ construct with RNA-Seq for all time points in the octanoic acid producer strain and secondly, the expression of the marker gene on the plasmid, HIS3, was increased accordingly. In summary, this suggests that gene expression is not optimized for a maximum production of octanoic acid but rather ideal for producing sufficient long-chain fatty acids to survive and mitigate toxic effects of octanoic acid. This could also explain the limit in octanoic acid production that we seem to encounter, as the precursor malonyl-CoA is increasingly used for $\mathrm{C} 16$ and $\mathrm{C} 18$ rather than for C8 fatty acid biosynthesis.

To get a deeper understanding of transcriptional changes, we analyzed differential expression at the three sampling times in the producer compared to the reference. For this comparison, we chose a $\log _{2} \mathrm{FC}$ of at least 1 as a threshold $(\mathrm{FDR}<0.01)$ and chose a subset of the hits for further analysis. We evaluated the effect of additional, plasmid-based expression of all genes that were found to be up- or downregulated, respectively, at more than one RNA-Seq sampling time in the 
octanoic acid producer. For this purpose, we chose to use a multi-copy library which contains the entire yeast genome cloned onto $2 \mu$ backbone plasmids and each plasmid contains roughly 2-10 genes. ${ }^{16}$ Even though the other genes on the plasmid might also affect octanoic acid production, it is unlikely that they so much influence titers as to mask the possible effect of the gene-ofinterest. The expression of HXT2, BTN2, IMD2 and ECI1 from library plasmids led to a decrease in octanoic acid titers after $46 \mathrm{~h}$ of fermentation whereas individual knockouts did not have any effect on growth or octanoic acid titers (Figure 4). Interestingly, growth and octanoic acid production were also delayed as a result of HXT2, BTN2 and IMD2 overexpressions (Figure 3). In our RNA-Seq data, HXT2 was found significantly downregulated at all sampling times. In addition, HXT2 expression has also been found downregulated with externally supplied octanoic acid. ${ }^{13}$ As the downregulation was observable at all three sampling times, it seems to be rather independent of octanoic acid production. The expression of BTN2, similarly to HXT2, was downregulated at all sampling times in RNA-Seq $\left(\log _{2} \mathrm{FC}>1, \mathrm{FDR}<0.01\right.$; except at $\mathrm{T2}$ : $\left.\log _{2} \mathrm{FC}=0.7\right)$ and its additional plasmid-based expression led to lower octanoic acid titers. BTN2 encodes a v-SNARE protein involved in intracellular protein transport and regulation of $\mathrm{pH}^{21,22}$ In another study, $\Delta b t n 2$ strains showed an enhanced activity of vacuolar $\mathrm{H}^{+}$-ATPase as well as diminished growth and decreased buffering capacity under sorbic acid supply, affecting intracellular $\mathrm{pH}$ homeostasis. ${ }^{21}$ This contradicts the downregulation of BTN2 expression at all sampling times in the octanoic acid producer, as the presence of octanoic acid might create more acidic conditions which the cell has to counteract. Also, our $\Delta b t n 2$ strain did not show any difference in octanoic acid titers. The reason for the decrease in octanoic acid titers following plasmid-based BTN2 expression is also unclear. These partly contradictory results could also arise from differences in protein turnover or possible post-translational modifications. The expression of IMD2 was significantly upregulated in our RNASeq data at all three sampling times $\left(\log _{2} F C>1\right.$, FDR $<0.01$; except at T1 $\left.\log _{2} F C=0.65\right)$ and additional plasmid-based expression led to a $13 \%$ decrease in octanoic acid titers but did not influence growth. IMD2 encodes an inosine monophosphate dehydrogenase, an enzyme involved in GTP biosynthesis. For $E C l 1$, we observed a significant upregulation of expression at $\mathrm{T} 3\left(\log _{2} \mathrm{FC}=\right.$ 1.4) in our data as well as under octanoic acid supply in a non-producer strain in previously published data. ${ }^{13} \mathrm{ECl} 1$ encodes a peroxisomal isomerase and is essential for $\beta$-oxidation of unsaturated fatty acids. ${ }^{23}$ We observed that ECI1 plasmid-based expression resulted in less octanoic acid whereas its knockout (LBY55) showed similar titers as the reference strain. We suspect that upregulation of $E C I 1$ leads to increased $\beta$-oxidation of unsaturated fatty acids, thereby possibly covering the cell's need for acetyl-CoA. Why this also leads to lower octanoic acid titers, remains obscure, as Eci1p has not been described to be involved in medium-chain fatty acid 
degradation. The exact effect of all four enzymes on octanoic acid biosynthesis remains to be investigated.

We found that most genes identified to be differentially expressed at more than one sampling time, did not have a significant effect on octanoic acid titers when overexpressed. This suggests that the upregulation of these genes in the octanoic acid producer had effects not directly related to the octanoic acid titers. One possibility is that the observed gene expression changes help to mitigate effects on precursor availability or for instance toxic effects of the acid.

The library plasmid containing two genes of interest, namely RPL4OB and PTR2, led to a strong increase in octanoic acid titer after $46 \mathrm{~h}$ of fermentation. Further analysis showed that plasmidbased expression of RPLAOB was responsible for the improvement. Interestingly, secreted C18:0 fatty acid titers were halved in this strain. As RPLAOB expression was downregulated at T3 in the transcriptomics analysis $\left(\log _{2} \mathrm{FC}=0.45\right)$, we suspect that this decrease reduces toxicity by lowering octanoic acid synthesis. In addition, a decrease in RPL4OB expression may help maintain long-chain fatty acid synthesis at sufficient levels. Consequently, this would increase the strain's fitness. The genomic integration of a second copy of RPL4OB under the control of the strong $P P G K 1$ promoter did not increase octanoic acid titers significantly and had no effect on C18 fatty acid titers. It seems that genomic expression of a second gene copy was not strong enough to exhibit the effects observed with expression from a multi-copy plasmid. Intriguingly, RPL4OB expression from a multicopy plasmid in non-producer CEN.PK2-1C, resulted in reduced growth. It is possible that this negative effect on growth is only observable in CEN.PK2-1C because LBY38 is already growth inhibited by inherent octanoic acid production. The decreased growth resulting from RPL4OB overexpression in CEN.PK2-1C could also be attributed to lowered C18 fatty acid production by the native FAS complex and thereby impaired robustness. Rpl40b is a subunit of the ribosome ${ }^{24}$ but to our knowledge has never been reported to play a direct role in fatty acid biosynthesis. Future work could investigate the global effect of increased Rpl40b expression via ribosome profiling ("RiboSeq"), ${ }^{25}$ proteome analyses and ribosome assembly studies. Another interesting approach could be to study knockout or knockdown mutants of $R P L 4 O B$ or of other ribosomal subunits such as RPL4OA

In the aforementioned published transcriptomic data sets, ${ }^{12,13}$ which were generated by adding octanoic acid externally to $S$. cerevisiae wild type strains, RPLAOB had not been found to be differentially regulated. Overall, there was little overlap between our RNA-Seq data set and the two other data sets. On the one hand, the diverging results could be attributed to the difference in experimental conditions, such as strain background, media, and $\mathrm{pH}$. More importantly, 
differential expression seems to be highly growth phase-dependent and to differ for internally produced and externally supplied octanoic acid. This underlines the importance of collecting transcriptomic data not just for externally added compounds, but for actual producer strains. Our comprehensive transcriptomics data set facilitated the detection of an unreported genetic target by enabling an in-depth view into yeast physiology during octanoic acid production. We were able to identify the new target $R P L 4 O B$, overexpression of which led to $40 \%$ more octanoic acid.

\section{MATERIALS AND METHODS}

Strains and Plasmid Construction. Yeast strains and plasmids used throughout this study are listed in Table S1. The genes DAN1 and NOP56 for overexpression were amplified from SHY $34{ }^{26}$ genomic DNA with primers containing the respective overhangs for cloning via Golden Gate ${ }^{27}$ (oligonucleotides are listed in Table S2). The PDR12-tPDR12 fragment as well as promoter $p T D H 3$ were amplified from CEN.PK113-11C genomic DNA with primers containing the respective overhangs for cloning via homologous recombination into pRS42H/EcoRV. The library plasmid containing RPL4OB and PTR2 (A10-C10 from ${ }^{16}$ ) was split into two plasmids as follows: Each gene was amplified with its endogenous up- and downstream regions from the original plasmid with primers containing overhangs for the pGP564 backbone for homologous recombination in yeast (CEN.PK2-1C). pGP564 was digested with BamHI/PstI prior to use. Genomic knockout of HXT2 was performed via CRISPR/Cas9 as described previously. ${ }^{28}$ The CRISPR/Cas9 plasmid for $h x t 2$ knockout was assembled via Golden Gate - SiHV138 was used and the GFP-dropout region was replaced by pre-assembled, double stranded gRNA oligonucleotides. For BTN2, IMD2 and ECI1 knockout via CRISPR/Cas9 was not possible and ORFs were knocked out with the Cre-loxP recombinase system with the $H P H^{R}$ marker as described in ${ }^{29}$. For this purpose, the resistance cassette was amplified from plasmid pUG6H with primers containing overhangs for the up- and downstream regions, respectively, of the targeted ORF. For genomic integration of a second copy of RPL4OB, the promoter $P P G K 1$ was amplified via PCR from PYTK011 and RPL4OB was amplified via PCR from the original plasmid including the downstream part. Used primers contained suitable overhangs for the parts or PYK2 up- or downstream regions, respectively. Integration was achieved via CRISPR/Cas9 plasmid LBV103 (assembled via homologous recombination into pRCC-K backbone) into the PYK2 locus, thereby removing the entire PYK2 ORF. Yeast transformations were performed according to Gietz and Schiest| ${ }^{30}$ or for SHY34 via an adapted procedure. ${ }^{9}$ Cells were streaked out on selective YPD ( 1 \% yeast extract, 2 \% peptone, both by BD, Difco Laboratories, USA; $2 \%$ D-glucose, Roth, Germany) containing hygromycin $\left(200 \mu \mathrm{g} \mathrm{mL}^{-1}\right)$ to select for $h p h N T 1, \mathrm{G}$ $418\left(200 \mu \mathrm{g} \mathrm{mL}^{-1}\right)$ to select for KanMX or on selective SCD medium ${ }^{17}$ lacking leucine $\left(\mathrm{SCD}^{\text {leu }}\right)$ to 
select for LEU2. Electrocompetent E. coli DH10ß (Gibco BRL, Gaithersburg, MD) was used for subcloning according to standard procedures, and transformants were selected on lysogeny broth (LB) agar plates ${ }^{31}$ supplemented with carbenicillin $\left(100 \mu \mathrm{g} \mathrm{mL}^{-1}\right)$, chloramphenicol $\left(20 \mu \mathrm{g} \mathrm{mL}^{-1}\right)$ or kanamycin $\left(50 \mu \mathrm{gL}^{-1}\right)$.

Cultures for RNA-Seq analysis. For pre-cultures, several colonies of SHY34 with plasmid fusFAS ${ }^{\text {wt }}$ or fus $F A S^{R K}$, respectively, were inoculated in $20 \mathrm{~mL}$ YPD with $100 \mathrm{mM}$ potassium phosphate buffer adjusted to $\mathrm{pH} 6.5$ and shaken (180 r.p.m.) at $30^{\circ} \mathrm{C}$ overnight. The main cultures were inoculated in triplicates to an $\mathrm{OD}_{600}$ of $0.1 \mathrm{in} 50 \mathrm{~mL}$ buffered YPD medium in $300 \mathrm{ml}$ flasks on a cell growth quantifier (aquila biolabs $\mathrm{GmbH}$, Germany ${ }^{17}$ ). Samples were taken for fatty acid quantification as described below. For RNA extraction, 10 OD of cells/sample were pelleted in a pre-cooled falcon at $4^{\circ} \mathrm{C}$ (3000 rcf) for $3 \mathrm{~min}$. Falcons were then immediately dropped into liquid nitrogen for a few minutes and stored at $-80^{\circ} \mathrm{C}$ until further processing.

RNA extraction, processing and sequencing. RNA extractions were performed on samples that were mechanically lysed with $0.5 \mathrm{~mm}$ acid washed beads using an MP-Biomedicals ${ }^{\mathrm{TM}}$ FastPrep-24 for three one-minute cycles. Further extraction was performed using an RNeasy ${ }^{\circledR}$ Kit from Qiagen. Libraries were prepared using the TrueSeq mRNA Stranded HT kit. Sequencing was carried out using an Illumina NextSeq 500 High Output Kit v2 (75 cycles), with a minimum of 10 million singleend reads per replicate. The Novo Nordisk Foundation Centre for Biosustainability (Technical University of Denmark) performed the RNA sequencing and library preparation. RNA-Seq data sets can be found using SRA accession PRJNA575618. RNA-Seq read mapping was performed after analysis in FASTQC. RNA-Seq mapping for differential expression was mapped with STAR ${ }^{32}$ and reads were assigned with featureCounts ${ }^{33}$. Differential expression results can be found in Supplementary File 1.

Cultures for Fatty Acid Production. S. cerevisiae strains were grown as previously described ${ }^{9}$ with minor adjustments. For pre-cultures, several colonies of a strain were inoculated in $20 \mathrm{~mL}$ YPD with $100 \mathrm{mM}$ potassium phosphate buffer adjusted to $\mathrm{pH} 6.5$ and antibiotic (200 $\mu \mathrm{g} \mathrm{mL}^{-1}$ hygromycin), if applicable, or in similarly buffered SCD medium lacking leucine ( $\left.S C D^{\text {-leu}}\right)$. After shaking (180 r.p.m.) at $30{ }^{\circ} \mathrm{C}$ overnight, the main culture was inoculated to an $\mathrm{OD}_{600}$ of 0.1 in the respective medium and cultured in $300 \mathrm{~mL}$ shake flasks under the same conditions. For sampling, the cultures were harvested by centrifugation and $10 \mathrm{~mL}$ of the supernatant was used for fatty acid extraction.

Fatty Acid Extraction and Derivatization. Fatty acid extraction and derivatization were performed as described previously. ${ }^{3}$ Cells were separated from the medium (3500 rcf, $10 \mathrm{~min}$ ), an internal 
standard ( $0.2 \mathrm{mg}$ heptanoic acid) was added to $10 \mathrm{~mL}$ supernatant and mixed with $1 \mathrm{~mL} 1 \mathrm{M} \mathrm{HCl}$ and $2.5 \mathrm{~mL}$ methanol/chloroform solution (1:1). After vigorous shaking for $3 \mathrm{~min}$, the mixture was centrifuged at $3000 \mathrm{rcf}$ for $10 \mathrm{~min}$ and the chloroform layer was recovered and evaporated overnight. The methylation of the fatty acids was performed as previously described. ${ }^{34}$ Samples were dissolved in $200 \mu \mathrm{L}$ toluene, mixed with $1.5 \mathrm{~mL}$ methanol and $300 \mu \mathrm{L} 8.0 \%(\mathrm{w} / \mathrm{v}) \mathrm{HCl}$ solution (diluted in methanol), vortexed, and incubated at $100{ }^{\circ} \mathrm{C}$ for $3 \mathrm{~h}$ to form fatty acid methyl esters (FAME). After cooling on ice for $10 \mathrm{~min}, 1 \mathrm{~mL} \mathrm{H} \mathrm{H}_{2} \mathrm{O}$ and $1 \mathrm{~mL}$ hexane were added to the sample, followed by thorough shaking, and the organic phase was transferred to a GC vial.

GC-FID analysis of FAMEs. GC analyses were carried out on a Perkin Elmer Clarus 400 instrument (Perkin Elmer, Germany) equipped with an Elite FFAP capillary column (30 m $\times 0.25 \mathrm{~mm}$, film thickness: $0.25 \mu \mathrm{m}$; PerkinElmer, Germany) and a flame ionization detector (Perkin Elmer, Germany) as described previously. ${ }^{3}$

Toxicity test. CEN.PK2-1C was transformed with plasmid pGP564 or RPL4OB, respectively, and plated on $\mathrm{SCD}^{\text {-leu }}$. Pre-cultures were inoculated in triplicates in buffered $\mathrm{SCD}^{- \text {leu }}$ and grown over night at $30{ }^{\circ} \mathrm{C}$ (180 r.p.m.). For main cultures, strains were inoculated to an $\mathrm{OD}_{600}$ of 0.2 and incubated for $4 \mathrm{~h}$ under the same condition ( $30^{\circ} \mathrm{C}, 180$ r.p.m.). The cultures were then diluted in fresh media to an $\mathrm{OD}_{600}$ of 0.05 and transferred into a 96 -well plate (clear with flat bottom) with $50 \mu \mathrm{l}$ per well. A dilution series was made with octanoic acid diluted in the same media, to reach final concentrations in the wells of 0-300 mg/L. Of these dilutions, $200 \mu \mathrm{l}$ were added per well. The three biological replicates per strain were inoculated in technical triplicates in the well plates. The starting absorbance was measured in a platereader (CLARIOstar ${ }^{\circledast}$, BMG Labtech, Ortenberg, Germany) and plates were incubated for $20 \mathrm{~h}$ at $30{ }^{\circ} \mathrm{C}$ without shaking before absorbance was measured again. From final absorbance values, a blank value (media without strain) was subtracted.

Software. RNA-Seq read mapping and data analyses were performed with R packages limma, edgeR and piano. Data tables were stored in Microsoft Excel 2016. Fermentation graphs were made using the software Prism 9 (GraphPad, USA). Geneious Prime 2020.2 software was used for assembly planning. 
Abbreviations. FAS: fatty acid synthase; FDR: false discovery rate; GC: gas chromatography; $\log _{2} \mathrm{FC}$ : $\log _{2}$ fold change; MPT: malonyl-palmitoyl transferase; $\mathrm{OD}_{600}$ : optical density at $\lambda=600 \mathrm{~nm}$.

Data availability. RNA-Seq data sets of data generated in this study can be found using SRA accession PRJNA575618.

\section{ASSOCIATED CONTENT}

\section{Supporting Information}

Supplementary Figures and Tables: Tables S1-S2 and Figures S1-S6

Supplementary File 1_differential expression

Supplementary File 2_GO term enrichment analysis

\section{AUTHOR INFORMATION}

\section{Corresponding Author}

*E-mail: m.oreb@bio.uni-frankfurt.de

\section{Author Contributions}

LB performed experiments including RNA-Seq fermentations, cloning, engineered strain fermentations and analysis. TD performed RNA extraction. LB and TD analysed RNA-Seq data and wrote the paper. MO and EB co-supervised the project. VS and JN provided advice and resources. All authors read and approved the manuscript.

\section{Conflicts of interest}

EB is inventor of EP patent application No. 15162192.7 filed on April 1, 2015, and of EP patent application No. 15174342.4 filed on June 26, 2015, by Goethe-University Frankfurt, concerning short-chain acyl-CoA producing FAS variants. There are no other competing interests.

\section{Funding}

This project has received funding from the European Union's Horizon 2020 research and innovation programme under grant agreement No. 720824. 


\section{REFERENCES}

(1) Huang, C. B., Alimova, Y., Myers, T. M., and Ebersole, J. L. (2011) Short- and medium-chain fatty acids exhibit antimicrobial activity for oral microorganisms. Arch. Oral Biol. 56, 650-654.

(2) Rosenblatt, J., Reitzel, R. A., Vargas-Cruz, N., Chaftari, A.-M., Hachem, R., and Raad, I. (2017) Caprylic and Polygalacturonic Acid Combinations for Eradication of Microbial Organisms Embedded in Biofilm. Front. Microbiol. 8, 1999.

(3) Henritzi, S., Fischer, M., Grininger, M., Oreb, M., and Boles, E. (2018) An engineered fatty acid synthase combined with a carboxylic acid reductase enables de novo production of 1-octanol in Saccharomyces cerevisiae. Biotechnol. Biofuels 11.

(4) Sarria, S., Kruyer, N. S., and Peralta-Yahya, P. (2017) Microbial synthesis of medium-chain chemicals from renewables. Nat. Biotechnol. 35, 1158-1166.

(5) Yan, Q., and Pfleger, B. F. (2020) Revisiting metabolic engineering strategies for microbial synthesis of oleochemicals. Metab. Eng. 58, 35-46.

(6) Schmidt, J. H. (2015) Life cycle assessment of five vegetable oils. J. Clean. Prod. 87, 130-138.

(7) Hu, Y., Zhu, Z., Nielsen, J., and Siewers, V. (2019) Engineering Saccharomyces cerevisiae cells for production of fatty acid-derived biofuels and chemicals. Open Biol. 9, 190049.

(8) Klug, L., and Daum, G. (2014) Yeast lipid metabolism at a glance. FEMS Yeast Res. 14, 369-388.

(9) Gajewski, J., Pavlovic, R., Fischer, M., Boles, E., and Grininger, M. (2017) Engineering fungal de novo fatty acid synthesis for short chain fatty acid production. Nat. Commun. 8, 14650.

(10) Baumann, L., Wernig, F., Born, S., and Oreb, M. (2020) Engineering Saccharomyces cerevisiae for production of fatty acids and their derivatives., in The Mycota Vol. II: Genetics and Biotechnology, 3rd edition (Benz, J. P., and Schipper, K., Eds.), pp 339-368. Springer, Switzerland.

(11) Jarboe, L. R., Liu, P., and Royce, L. A. (2011) Engineering inhibitor tolerance for the production of biorenewable fuels and chemicals. Curr. Opin. Chem. Eng. 1, 38-42.

(12) Legras, J. L., Erny, C., Le Jeune, C., Lollier, M., Adolphe, Y., Demuyter, C., Delobel, P., Blondin, B., and Karst, F. (2010) Activation of two different resistance mechanisms in Saccharomyces cerevisiae upon exposure to octanoic and decanoic acids. Appl. Environ. Microbiol. 76, 7526-7535.

(13) Liu, P., Chernyshov, A., Najdi, T., Fu, Y., Dickerson, J., Sandmeyer, S., and Jarboe, L. (2013) Membrane stress caused by octanoic acid in Saccharomyces cerevisiae. Appl. Microbiol. Biotechnol. 97, 3239-3251.

(14) Wernig, F., Born, S., Boles, E., Grininger, M., and Oreb, M. (2020) Fusing $\alpha$ and $\beta$ subunits of the fungal fatty acid synthase leads to improved production of fatty acids. Sci. Rep. 10, 9780.

(15) Leber, C., Choi, J. W., Polson, B., and Da Silva, N. A. (2016) Disrupted short chain specific $\beta$ oxidation and improved synthase expression increase synthesis of short chain fatty acids in Saccharomyces cerevisiae. Biotechnol. Bioeng. 113, 895-900.

(16) Jones, G. M., Stalker, J., Humphray, S., West, A., Cox, T., Rogers, J., Dunham, I., and Prelich, G. 
(2008) A systematic library for comprehensive overexpression screens in Saccharomyces cerevisiae. Nat. Methods 5, 239-241.

(17) Bruder, S., Reifenrath, M., Thomik, T., Boles, E., and Herzog, K. (2016) Parallelised online biomass monitoring in shake flasks enables efficient strain and carbon source dependent growth characterisation of Saccharomyces cerevisiae. Microb. Cell Fact. 15, 1-15.

(18) Viegas, C. A., Rosa, M. F., Sá-Correia, I., and Novais, J. M. (1989) Inhibition of Yeast Growth by Octanoic and Decanoic Acids Produced during Ethanolic Fermentation. Appl. Environ. Microbiol. $55,21-28$.

(19) Viegas, S. C., Sá-Correia, I., and Novais, J. M. (1985) Synergistic inhibition of the growth of Saccharomyces bayanus by ethanol and octanoic or decanoic acids. Biotechnol. Lett. 7, 611-614.

(20) Besada-Lombana, P. B., Fernandez-Moya, R., Fenster, J., and Da Silva, N. A. (2017) Engineering Saccharomyces cerevisiae fatty acid composition for increased tolerance to octanoic acid. Biotechnol. Bioeng. 114, 1531-1538.

(21) Chattopadhyay, S., Muzaffar, N. E., Sherman, F., and Pearce, D. A. (2000) The yeast model for batten disease: Mutations in btn1, btn2, and hsp30 alter pH homeostasis. J. Bacteriol. 182, 64186423.

(22) Chattopadhyay, S., Roberts, P. M., and Pearce, D. A. (2003) The yeast model for Batten disease: A role for Btn2p in the trafficking of the Golgi-associated vesicular targeting protein, Yif1p. Biochem. Biophys. Res. Commun. 302, 534-538.

(23) Geisbrecht, B. V., Zhu, D., Schulz, K., Nau, K., Morrell, J. C., Geraghty, M., Schulz, H., Erdmann, R., and Gould, S. J. (1998) Molecular characterization of Saccharomyces cerevisiae $\Delta^{3}, \Delta^{2}$-enoyl-CoA isomerase. J. Biol. Chem. 273, 33184-33191.

(24) Fernández-Pevida, A., Rodríguez-Galán, O., Díaz-Quintana, A., Kressler, D., and De La Cruz, J. (2012) Yeast ribosomal protein L40 assembles late into precursor $60 \mathrm{~S}$ ribosomes and is required for their cytoplasmic maturation. J. Biol. Chem. 287, 38390-38407.

(25) Weinberg, D. E., Shah, P., Eichhorn, S. W., Hussmann, J. A., Plotkin, J. B., and Bartel, D. P. (2016) Improved Ribosome-Footprint and mRNA Measurements Provide Insights into Dynamics and Regulation of Yeast Translation. Cell Rep. 14, 1787-1799.

(26) Wernig, F., Boles, E., and Oreb, M. (2019) De novo biosynthesis of 8-hydroxyoctanoic acid via a medium-chain length specific fatty acid synthase and cytochrome P450 in Saccharomyces cerevisiae. Metab. Eng. Commun. e00111.

(27) Lee, M. E., DeLoache, W. C., Cervantes, B., and Dueber, J. E. (2015) A Highly Characterized Yeast Toolkit for Modular, Multipart Assembly. ACS Synth. Biol. 4, 975-986.

(28) Generoso, W. C., Gottardi, M., Oreb, M., and Boles, E. (2016) Simplified CRISPR-Cas genome editing for Saccharomyces cerevisiae. J. Microbiol. Methods 127, 203-205.

(29) Güldener, U., Heck, S., Fiedler, T., Beinhauer, J., and Hegemann, J. H. (1996) A new efficient gene disruption cassette for repeated use in budding yeast. Nucleic Acids Res. 24, 2519-2524.

(30) Gietz, R. D., and Schiestl, R. H. (2007) High-efficiency yeast transformation using the LiAc/SS 
carrier DNA/PEG method. Nat. Protoc. 2, 31-34.

(31) Sambrook, J., Fritsch, E., and Maniatis, T. (1989) Molecular cloning: A laboratory manual. Second edition. Cold Spring Harb. Lab. Press. New York. (Evans, G. A., Ed.).

(32) Dobin, A., Davis, C. A., Schlesinger, F., Drenkow, J., Zaleski, C., Jha, S., Batut, P., Chaisson, M., and Gingeras, T. R. (2013) STAR: Ultrafast universal RNA-seq aligner. Bioinformatics 29, 15-21.

(33) Liao, Y., Smyth, G. K., and Shi, W. (2014) FeatureCounts: An efficient general purpose program for assigning sequence reads to genomic features. Bioinformatics 30, 923-930.

(34) Ichihara, K., and Fukubayashi, Y. (2010) Preparation of fatty acid methyl esters for gas-liquid chromatography. J. Lipid Res. 51, 635-640. 


\section{SUPPORTING INFORMATION}

Table S1. Yeast strains and plasmids used in this study.

\begin{tabular}{|c|c|c|}
\hline Strain & Characteristics & Reference \\
\hline CEN.PK2-1C & $\begin{array}{l}\text { MATa; MAL2-8c; SUC2; ura3-52; his3 } 1 \text {; } \\
\text { leu2-3_112; trp1-289 }\end{array}$ & $\begin{array}{l}\text { Euroscarf, } \\
\text { Frankfurt am } \\
\text { Main, Germany }\end{array}$ \\
\hline RPY21 & 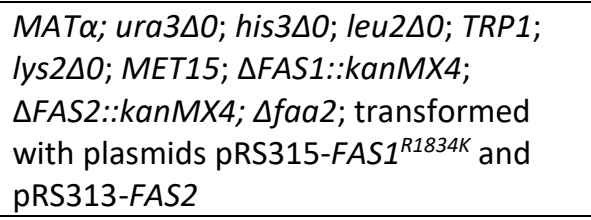 & 1 \\
\hline SHY34 & 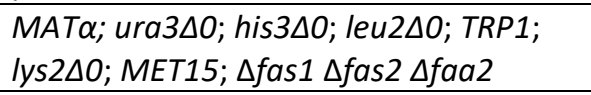 & 2 \\
\hline LBY38 & $\begin{array}{l}\text { CEN.PK2-1C } \triangle p F A S 1-1-300:: p H X T 7-1- \\
392 \triangle p F A S 2-1-200:: p H X T 7-1-392, \\
\text { FAS1 }{ }^{R 1834 K}, \triangle \text { faa2 }\end{array}$ & This group \\
\hline LBY49 & LBY38 $\Delta h x t 2$ & This study \\
\hline LBY53 & LBY38 $\Delta b t n 2$ & This study \\
\hline LBY55 & LBY38 $\Delta e c i 1$ & This study \\
\hline LBY61 & LBY38 $\Delta p y k 2:: p P G K 1-R P L 4 O B-t R P L 4 O B$ & This study \\
\hline Plasmid & Characteristics & Reference \\
\hline \multicolumn{3}{|l|}{ Plasmids used for fermentations } \\
\hline fusFAS ${ }^{w t}$ & pRS313-pFAS-fusFAS12-Wt & 3 \\
\hline fusFAS ${ }^{R K}$ & pRS313-pFAS-fusFAS12-RK & 3 \\
\hline $\mathrm{pRS} 42 \mathrm{H}$ & $\begin{array}{l}2 \mu, H P H^{R}, A m p R, \text { multiple cloning site } \\
\text { including EcoRV }\end{array}$ & 4 \\
\hline LBV16 (PDR12) & $\begin{array}{l}\text { pRS42H with } p T D H 3-P D R 12-t P D R 12 \\
\text { integrated in EcoRV site }\end{array}$ & This study \\
\hline pGP564 & $2 \mu, L E U 2$, KanR & 5 \\
\hline $\begin{array}{l}\text { Library plasmids (containing genes): } \\
\text { A10-F1 (FAS1), A16-E2 (FAS2), A15-A7 } \\
\text { (HIS3/YOR203W), A15-D6 (YOR186W), } \\
\text { A9-H11 (MCH2), A8-G4 (IMD2), A16-D6 } \\
\text { (EEB1), A12-F8 (HXT2), A1-E10 (MNC1), } \\
\text { A10-C10 (PTR2), A7-E2 (BTN2), A1-G6 } \\
\text { (URA7), A5-A2 (UTR2), A3-B11 } \\
\text { (YDR133C), A12-B2 (INA1), A14-D9 } \\
(\text { IZH4), A1-A8 (PDR3), A8-A2 (SPS100), } \\
\text { A13-E10 (SPS19), A11-C10 (ECI1), A15- } \\
\text { B2 (RSB1), A1-C1 (GDH3), A5-E11 } \\
(\text { HSP12), A10-C10 (RPL4OB/PTR2) }\end{array}$ & $\begin{array}{l}\text { pGP564, yeast genomic DNA fragments } \\
\text { of } \sim 10 \mathrm{~kb} \text { size }\end{array}$ & 5 \\
\hline LBV100 (PTR2) & $\begin{array}{l}\text { pGP564; } 2 \mu, \text { LEU2, KanR, pPTR2-PTR2- } \\
\text { tPTR2 }\end{array}$ & This study \\
\hline LBV101 (RPL40B) & $\begin{array}{l}\text { pGP564; } 2 \mu, L E U 2, K a n R, p R P L 4 O B- \\
\text { RPL40B-tRPL4OB }\end{array}$ & This study \\
\hline SiHV010 & $2 \mu, H P H^{R}, K a n R$ & 6 \\
\hline LBV71 & SiHV010-pYEF3-DAN1-tSSA1 & This study \\
\hline LBV72 & SiHV010-pYEF3-NOP56-tSSA1 & This study \\
\hline
\end{tabular}




\begin{tabular}{|c|c|c|}
\hline \multicolumn{3}{|c|}{ Plasmids used for CRISPR/Cas9 } \\
\hline pRCC-K & $\begin{array}{l}2 \mu, \text { kanMX } \text { AmpR, pROX3-Cas9opt_ } \\
\text { tCYC1, pSNR52-gRNA }\end{array}$ & 7 \\
\hline SiHV138 & $\begin{array}{l}2 \mu, \text { kanMX, AmpR, }_{\text {PROX3-Cas9opt }} \\
\text { tCYC1, pSNR52-gRNA, GFP-dropout } \\
\text { (modified from pRCC-K) }\end{array}$ & 7 \\
\hline LBV52 & $\begin{array}{l}\text { SiHV138 with } g R N A \\
\text { (TGGGTTAACCTTAGGACGTC) for HXT2 } \\
\text { locus }\end{array}$ & This study \\
\hline LBV103 & $\begin{array}{l}\text { pRCC-K with gRNA for PYK2 } \\
\text { (ATGTCTTTGGCGGACAAGGG) locus }\end{array}$ & This study \\
\hline \multicolumn{3}{|c|}{ Plasmid used for Golden Gate assembly } \\
\hline pYTK001 & Golden Gate entry vector, CamR & 6 \\
\hline \multicolumn{3}{|c|}{ Plasmids Cre-loxP recombinase system } \\
\hline pUG6H & $\begin{array}{l}\text { contains loxP-HPH} H^{R} \text {-loxP for knockout in } \\
\text { yeast }\end{array}$ & 8 \\
\hline
\end{tabular}

Table S2. Oligonucleotides used in this study.

\begin{tabular}{|c|c|c|}
\hline Primer & Sequence 5'-3' & Application \\
\hline \multicolumn{3}{|c|}{ Cloning of LBV71, LBV72 } \\
\hline LBP321 & CGTCTCGTCGGTCTCATATGTCTAGAATTAGTATATTAGCTGTCG & \multirow{2}{*}{$\begin{array}{l}\text { Amplification of DAN1 } \\
\text { from SHY34 genomic } \\
\text { DNA with overhangs for } \\
\text { Golden Gate cloning }\end{array}$} \\
\hline LPB322 & CGTCTCAGGTCGGTCTCAGGATCTATAACAATAGAGCGGCGGC & \\
\hline LBP323 & CGTCTCGTCGGTCTCATATGGCTCCTATTGAATACCTAC & \multirow{2}{*}{$\begin{array}{l}\text { Amplification of NOP56 } \\
\text { from SHY34 genomic } \\
\text { DNA with overhangs for } \\
\text { Golden Gate cloning }\end{array}$} \\
\hline LBP324 & $\begin{array}{l}\text { CGTCTCAGGTCGGTCTCAGGATTTAATCCTTACTTTTCTTCTTTTTA } \\
\text { TCCTTC }\end{array}$ & \\
\hline SiHSeq001 & TCCTGGCCTTTTGCTGG & \multirow{2}{*}{$\begin{array}{l}\text { Sequencing of DAN1 and } \\
\text { NOP56 in pYTK001 } \\
\text { backbone }\end{array}$} \\
\hline SiHSeq002 & GGACTCCTGTTGATAGATC & \\
\hline \multicolumn{3}{|c|}{ Cloning of LBV100, LBV101 } \\
\hline LBP406 & $\begin{array}{l}\text { CTCCACCGCGGTGGCGGCCGCTCTAGAACTAGTGTGCTATGTATT } \\
\text { CTTAGTGTACTGC }\end{array}$ & \multirow{2}{*}{$\begin{array}{l}\text { Amplification of PTR2 } \\
\text { including up- and } \\
\text { downstream regions } \\
\text { from A10-C10 of library }\end{array}$} \\
\hline LBP407 & $\begin{array}{l}\text { GTCGACGGTATCGATAAGCTTGATATCGAATTCTCGATTTATTAC } \\
\text { GATCTCCACAAATC }\end{array}$ & \\
\hline LBP408 & $\begin{array}{l}\text { GCTCCACCGCGGTGGCGGCCGCTCTAGAACTAGTGTGCGTTTAA } \\
\text { TTAACTTACTGTC }\end{array}$ & \multirow{2}{*}{$\begin{array}{l}\text { Amplification of } R P L 4 O B \\
\text { including up- and } \\
\text { downstream regions } \\
\text { from A10-C10 of library }\end{array}$} \\
\hline LBP409 & $\begin{array}{l}\text { CGAGGTCGACGGTATCGATAAGCTTGATATCGAATTCTATCCTAA } \\
\text { AACGTGGCGTTA }\end{array}$ & \\
\hline LBP237 & GGAATTGTGAGCGGATAAC & \multirow{2}{*}{$\begin{array}{l}\text { Sequencing of LBV100, } \\
\text { LBV101 }\end{array}$} \\
\hline LBP238 & ACTATAGGGCGAATTGGG & \\
\hline \multicolumn{3}{|c|}{ Cloning of LBV16 } \\
\hline LBP72 & $\begin{array}{l}\text { CCTCGAGGTCGACGGTATCGATAAGCTTGATATCACAGTTTATTC } \\
\text { CTGGCATCCAC }\end{array}$ & \multirow{2}{*}{$\begin{array}{l}\text { Amplification of } p P G K 1 \\
\text { from CEN.PK113-11C } \\
\text { with overhangs to PDR12 } \\
\text { and pRS42H, } \\
\text { respectively }\end{array}$} \\
\hline LBP73 & $\begin{array}{l}\text { GTCTTTCTCAATATGTTCGTCAGTCGAAGACATTTTGTTTGTTTAT } \\
\text { GTGTGTTTATTCG }\end{array}$ & \\
\hline LBP74 & $\begin{array}{l}\text { CGAATAAACACACATAAACAAACAAAATGTCTTCGACTGACGAA } \\
\text { CATATTGAGAAAGAC }\end{array}$ & $\begin{array}{l}\text { Amplification of PDR12- } \\
\text { tPDR12 from CEN.PK113- }\end{array}$ \\
\hline
\end{tabular}




\begin{tabular}{|c|c|c|}
\hline LBP75 & $\begin{array}{l}\text { GATCCCCCGGGCTGCAGGAATTCGATGATATCTCCTTTTTGAAGGT } \\
\text { GATAGGGATATAATG }\end{array}$ & $\begin{array}{l}11 \mathrm{C} \text { with overhangs to } \\
\text { pRS42H and } p T D H 3 \\
\text { respectively }\end{array}$ \\
\hline LBP85 & GGCGATTAAGTTGGGTAACG & Sequencing of LBV16 \\
\hline RPP056 & CACACAGGAAACAGCTATGAC & \\
\hline \multicolumn{3}{|c|}{ Knockout of $B T N 2, I M D 2$ and $E C I 1$ via Cre-loxP recombinase system } \\
\hline LBP325 & $\begin{array}{l}\text { CAACCAAAAGAAAATAACTAATAGACCCCATTACAATATAGAATT } \\
\text { CGTACGCTGCAGGTCGAC }\end{array}$ & \multirow{2}{*}{$\begin{array}{l}\text { Amplification of } H P H^{R} \\
\text { cassette with overhangs } \\
\text { for } B T N 2 \\
\text { up/downstream regions }\end{array}$} \\
\hline LBP326 & $\begin{array}{l}\text { CGTAAAAATGAAAGATGGGGAGTATGTATTATCACCCAGCATAG } \\
\text { GCCACTAGTGGATCTG }\end{array}$ & \\
\hline LBP329 & $\begin{array}{l}\text { ATAATCAGTGCAATTAATAACTCCACAAGTAGCAAAAGCATTCGT } \\
\text { ACGCTGCAGGTCGAC }\end{array}$ & \multirow{2}{*}{$\begin{array}{l}\text { Amplification of } H P H^{R} \\
\text { cassette with overhangs } \\
\text { for } I M D 2 \\
\text { up/downstream regions }\end{array}$} \\
\hline LBP330 & $\begin{array}{l}\text { CATCAGTAATACTGTATTGATGATGCCATTTTAACATGCATAGGC } \\
\text { CACTAGTGGATCTG }\end{array}$ & \\
\hline LBP333 & $\begin{array}{l}\text { GGCACAAATTGCTCGCACAGTAAAGGAAGGAAGAACAATTTCGT } \\
\text { ACGCTGCAGGTCGAC }\end{array}$ & \multirow{2}{*}{$\begin{array}{l}\text { Amplification of } H P H^{R} \\
\text { cassette with overhangs } \\
\text { for } E C I 1 \text { up/downstream } \\
\text { regions }\end{array}$} \\
\hline LBP334 & $\begin{array}{l}\text { ATTGTGTGTGCGTTTTGTTTCACTGAGAAAGCGGACGGCATAGG } \\
\text { CCACTAGTGGATCTG }\end{array}$ & \\
\hline LBP309 & GTCATGTAGCACTATTTCAGCC & \multirow{4}{*}{$\begin{array}{l}\text { Sequencing of BTN2 } \\
\text { locus }\end{array}$} \\
\hline LBP310 & GAATCACTTGCCATTCCACC & \\
\hline LBP327 & ACCATTACAGACCAACTTAC & \\
\hline LBP328 & AACTTCCTCATCCTCAAC & \\
\hline LBP311 & AGAGATGATGCAAGAGTAG & \multirow{4}{*}{$\begin{array}{l}\text { Sequencing of IMD2 } \\
\text { locus }\end{array}$} \\
\hline LBP312 & TCACCACCAAAATCACC & \\
\hline LBP331 & ACCAAGAGCCTACCAAGAC & \\
\hline LBP332 & CCAACATACCACCCATCATAAC & \\
\hline LBP335 & TCACTTAATGAACCCTGAC & \multirow[t]{4}{*}{ Sequencing of $E C l 1$ locus } \\
\hline LBP336 & СТСТССАТСТАСССАATAC & \\
\hline LBP337 & TCAAATAAACCTGCTGCC & \\
\hline LBP338 & ACGCAACTAAATGAGCAC & \\
\hline \multicolumn{3}{|c|}{ Knockout of $H X T 2$} \\
\hline LBP317 & GATCTGGGTTAACCTTAGGACGTC & \multirow{2}{*}{$\begin{array}{l}\text { Amplification of gRNA } \\
\text { for HXT2 locus }\end{array}$} \\
\hline LBP318 & AAACGACGTCCTAAGGTTAACCCA & \\
\hline LBP320 & $\begin{array}{l}\text { GATTATAAGAACAACAAATTAAATTACAAAAAGACTTATAAAGCA } \\
\text { ACATAGAGATTATACTTAAACTAGCACTGATTTTTTTTAAGGCTAAT } \\
\text { GGCTACTAA }\end{array}$ & Donor for $h x t 2$ knockout \\
\hline MGP126 & GGGAAACGCCTGGTATC & Sequencing of gRNAs \\
\hline LBP313 & TCCCTCTCCACTCTTTCTC & \multirow{2}{*}{$\begin{array}{l}\text { Sequencing of } H X T 2 \\
\text { locus }\end{array}$} \\
\hline LBP314 & ATCAGCCACAATACGCC & \\
\hline \multicolumn{3}{|c|}{ Integration of $p P G K 1-R P L 4 O B$ into $P Y K 2$} \\
\hline LBP106 & $\begin{array}{l}\text { ATGTCTTTTGGCGGACAAGGGGTTTTAGAGCTAGAAATAGCAAGT } \\
\text { TAAAATAAGG }\end{array}$ & \multirow[t]{2}{*}{$\begin{array}{l}\text { Amplification of gRNA } \\
\text { for } P Y K 2 \text { locus }\end{array}$} \\
\hline LBP107 & CCCTTGTCCGCCAAAGACATGATCATTTATCTTTCACTGCGGAG & \\
\hline LBP430 & $\begin{array}{l}\text { CATATTATTTCGAACATAGTATTCTGTATTCGCCGTAACATACATT } \\
\text { TGCATGATCTTGTTTTATATTTGTTGTAAAAAG }\end{array}$ & \multirow{2}{*}{$\begin{array}{l}\text { Amplification of } p P G K 1 \\
\text { from pYTK001 with } \\
\text { overhangs to } p y k 2- \\
\text { downstream and } \\
R P L 40 B \text {, respectively } \\
\end{array}$} \\
\hline LBP431 & $\begin{array}{l}\text { CTGACACAATGGACAATTAAATAAAATTAAGTAAAAAAAATAAG } \\
\text { GACTTTAATTTTTAAACGGTGAGTAAGGAAAG }\end{array}$ & \\
\hline LBP428 & $\begin{array}{l}\text { CTATATTTTACTTTCATCCTCTACGTCCATTGTAAGATTACAACAA } \\
\text { AAGCACTATCGTGCGTTTAATTAACTTACTGTC }\end{array}$ & \multirow{2}{*}{$\begin{array}{l}\text { Amplification of } R P L 4 O B \\
\text { with downstream region } \\
\text { from A10-C10 library }\end{array}$} \\
\hline LBP429 & $\begin{array}{l}\text { CAGATCATCAAGGAAGTAATTATCTACTTTTTACAACAAATATAA } \\
\text { AACAAGATCATGCAAATGTATGTTACGG }\end{array}$ & \\
\hline
\end{tabular}




\begin{tabular}{|l|l|l|}
\hline LBP118 & CAGAGCGGTGAAACGCAAC & $\begin{array}{l}\text { Sequencing of } \\
\text { pyk2::pPGK1-RPL4OB- } \\
\text { LRPL4OB }\end{array}$ \\
\cline { 1 - 2 } LBP119 & CGCAGTTTGCGAACATTACCTG & \\
\hline
\end{tabular}

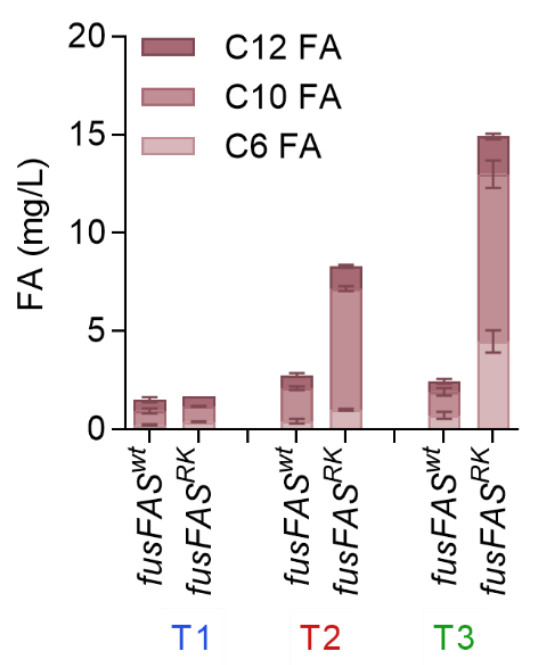

Figure S1. Characterization of C6, C10 and C12 fatty acid production. Strains SHY34+fusFAS ${ }^{w t}$ (reference strain) and SHY34+fusFAS ${ }^{R K}$ (octanoic acid producer) were cultured for $46 \mathrm{~h}$ in buffered YPD medium. Sampling times are indicated with arrows (T1 $=14 \mathrm{~h} ; \mathrm{T} 2=22 \mathrm{~h} ; \mathrm{T} 3=46 \mathrm{~h})$. Fatty acids (FA) were extracted and quantified via GC. $n=3$, error bars $= \pm$ standard deviation. 


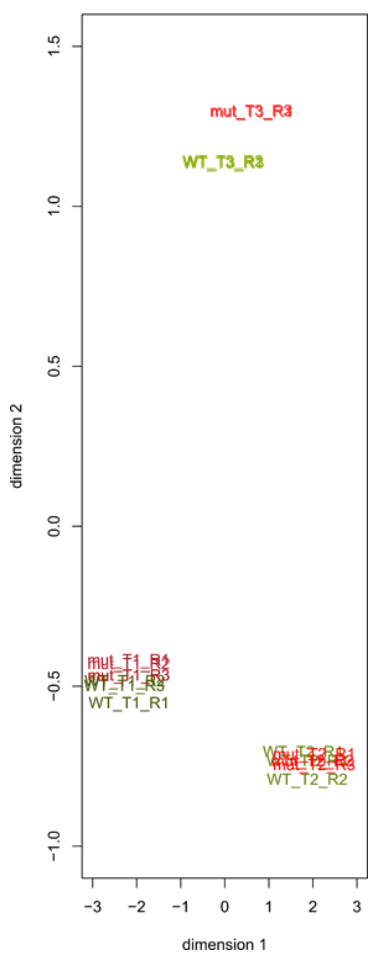

Figure S2. Principal component analysis (PCA) of RNASeq data sets. The RNASeq data of all three replicates (R1-R3) of the three sampling times (T1 = 14 h; T2 = 22 h; T3=46 h) of the reference strain (WT) and the octanoic acid producer strain (mut), respectively, were compared in PCA suggesting similar global expression.

T1

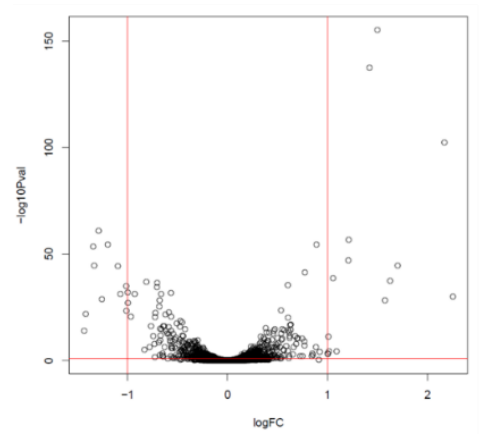

T2

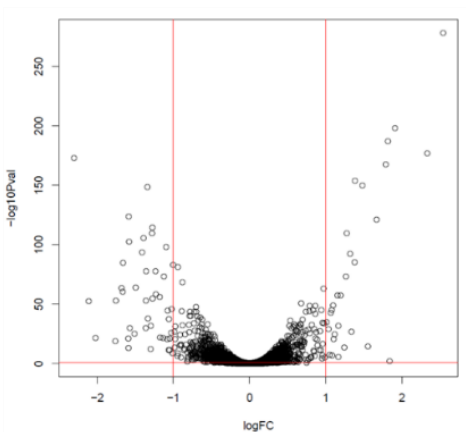

T3

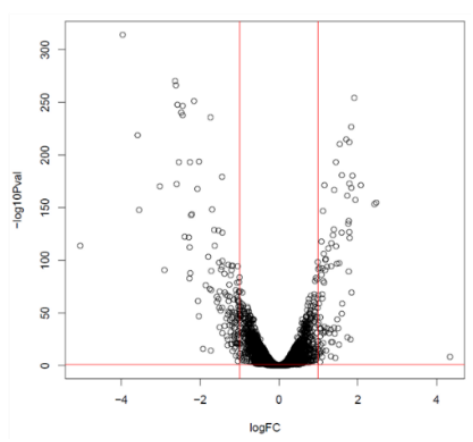

Figure S3. Differential gene expression analysis. Volcano plots show the $\log _{2} \mathrm{FC}$ of gene expression at the three sampling times (T1 = $14 \mathrm{~h} ; \mathrm{T} 2=22 \mathrm{~h}$; T3 $=46 \mathrm{~h}$ ) for SHY34+fusFAS ${ }^{R K}$ (octanoic acid producer) compared to SHY34+fUSFAS ${ }^{W T}$ (reference strain). 
(A) Upregulated
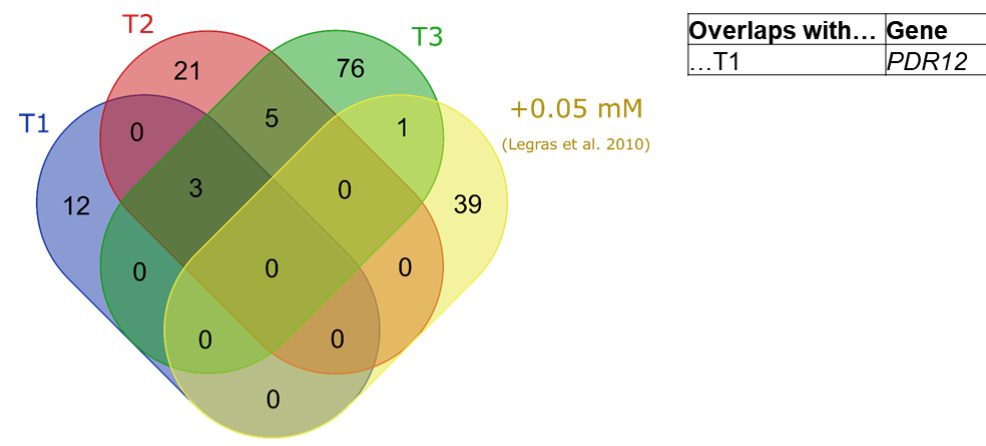

(B) Downregulated

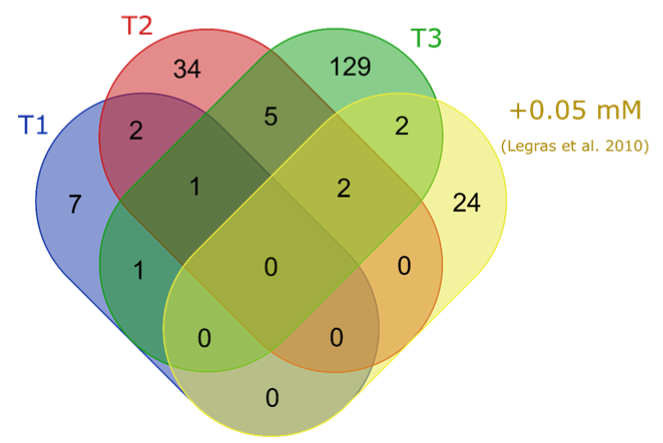

\begin{tabular}{|l|l|}
\hline Overlaps with... & Gene \\
\hline \multirow{2}{*}{. T2, T3 } & URA7 \\
\hline \multirow{2}{*}{$\ldots$ T3 } & INA1 \\
\hline ZRT1 \\
\hline
\end{tabular}

Figure S4. Comparison of transcriptome data of an octanoic acid producer with the response to externally supplied octanoic acid to a non-producer. Number of genes that are upregulated (A) or downregulated (B) in the two experiments. For RNA-Seq, an octanoic acid producer strain was analyzed in comparison to a non-producer strain $\left(\log _{2} \mathrm{FC}>1\right)$ at different sampling times ( $T 1=14$ $\mathrm{h} ; \mathrm{T} 2=22 \mathrm{~h} ; \mathrm{T} 3=46 \mathrm{~h}$ ). Microarray differential expression data of a non-producer strain supplied with $0.05 \mathrm{mM}$ octanoic acid for 20 minutes in comparison to non-supplied control $\left(\log _{2} \mathrm{FC} \geq 0.3\right.$ ); data taken from Legras et al. 2010. ${ }^{9}$ Gene lists include genes that overlap between data from Legras et al. 2010 and at least one sampling time of the RNA-Seq experiment. 
(A) Upregulated
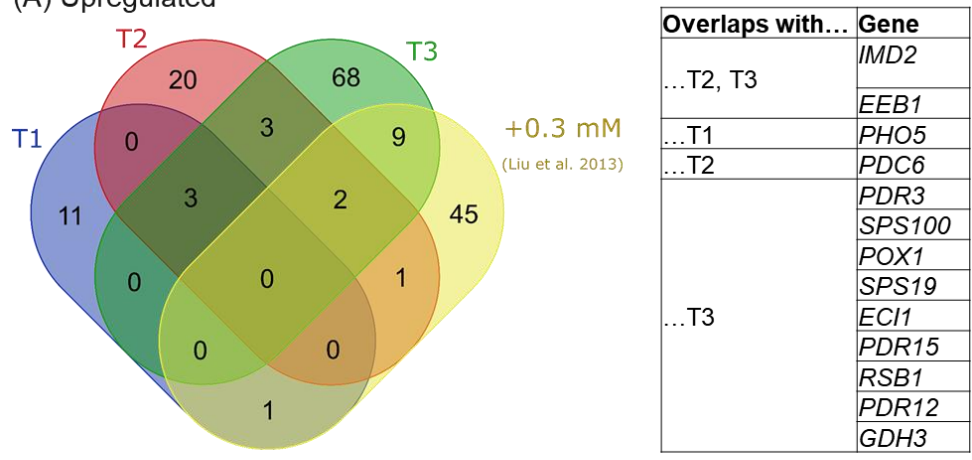

(B) Downregulated

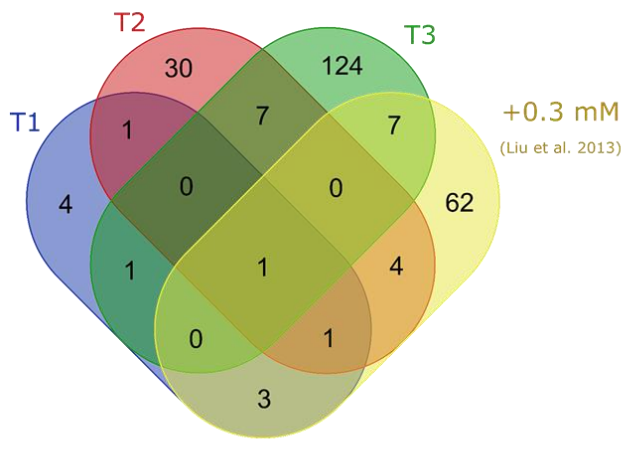

\begin{tabular}{|c|c|}
\hline Overlaps with... & Gene \\
\hline$\ldots \mathrm{T} 1, \mathrm{~T} 2, \mathrm{~T} 3$ & HXT2 \\
\hline$. \mathrm{T} 1, \mathrm{~T} 2$ & PTR2 \\
\hline \multirow{3}{*}{...T1 } & SPL2 \\
\hline & PHO84 \\
\hline & MMP1 \\
\hline \multirow{4}{*}{$\ldots \mathrm{T} 2$} & AGP1 \\
\hline & HXT4 \\
\hline & GAP1 \\
\hline & PUT4 \\
\hline \multirow{7}{*}{$\ldots \mathrm{T} 3$} & $D B P 2$ \\
\hline & SUR7 \\
\hline & $\mathrm{HO}$ \\
\hline & ARO9 \\
\hline & RNH201 \\
\hline & HSP12 \\
\hline & ARO10 \\
\hline
\end{tabular}

Figure S5. Comparison of transcriptome data of an octanoic acid producer with the response to externally supplied octanoic acid to a non-producer. Number of genes that are upregulated (A) or downregulated (B) in the two experiments. For RNA-Seq, an octanoic acid producer strain was analyzed in comparison to a non-producer strain $\left(\log _{2} \mathrm{FC}>1\right)$ at different sampling times ( $\mathrm{T} 1=14$ $\mathrm{h} ; \mathrm{T} 2=22 \mathrm{~h} ; \mathrm{T} 3=46 \mathrm{~h}$ ). Microarray differential expression data of a non-producer strain supplied with $0.3 \mathrm{mM}$ octanoic acid in comparison to non-supplied control $\left(\log _{2} \mathrm{FC}>1\right)$ in mid-log growth; data taken from Liu et al. 2013. ${ }^{10}$ Gene lists include genes that overlap between data from Liu et al. 2013 and at least one sampling time of the RNA-Seq experiment.

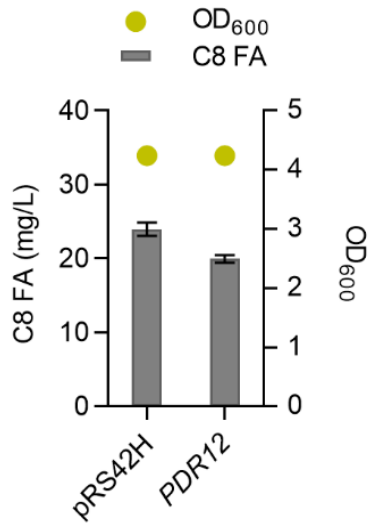

Figure S6. Effect of PDR12 overexpression. The octanoic acid producer strain RPY21-FAS1 ${ }^{R K}{ }_{-F A S 2}$ was transformed with a multi-copy plasmid containing PDR12 or the control vector pRS42H. Strains were cultured in buffered YPD hygro medium and fatty acids were extracted after $72 \mathrm{~h}$ and quantified by GC measurement. $n=2$, error bars $= \pm$ standard deviation. 


\section{REFERENCES}

(1) Henritzi, S., Fischer, M., Grininger, M., Oreb, M., and Boles, E. (2018) An engineered fatty acid synthase combined with a carboxylic acid reductase enables de novo production of 1-octanol in Saccharomyces cerevisiae. Biotechnol. Biofuels 11.

(2) Wernig, F., Boles, E., and Oreb, M. (2019) De novo biosynthesis of 8-hydroxyoctanoic acid via a medium-chain length specific fatty acid synthase and cytochrome P450 in Saccharomyces cerevisiae. Metab. Eng. Commun. e00111.

(3) Wernig, F., Born, S., Boles, E., Grininger, M., and Oreb, M. (2020) Fusing $\alpha$ and $\beta$ subunits of the fungal fatty acid synthase leads to improved production of fatty acids. Sci. Rep. 10, 9780.

(4) Taxis, C., and Knop, M. (2006) System of centromeric, episomal, and integrative vectors based on drug resistance markers for Saccharomyces cerevisiae. Biotechniques 40, 73-78.

(5) Jones, G. M., Stalker, J., Humphray, S., West, A., Cox, T., Rogers, J., Dunham, I., and Prelich, G. (2008) A systematic library for comprehensive overexpression screens in Saccharomyces cerevisiae. Nat. Methods 5, 239-241.

(6) Lee, M. E., DeLoache, W. C., Cervantes, B., and Dueber, J. E. (2015) A Highly Characterized Yeast Toolkit for Modular, Multipart Assembly. ACS Synth. Biol. 4, 975-986.

(7) Generoso, W. C., Gottardi, M., Oreb, M., and Boles, E. (2016) Simplified CRISPR-Cas genome editing for Saccharomyces cerevisiae. J. Microbiol. Methods 127, 203-205.

(8) Güldener, U., Heck, S., Fiedler, T., Beinhauer, J., and Hegemann, J. H. (1996) A new efficient gene disruption cassette for repeated use in budding yeast. Nucleic Acids Res. 24, 2519-2524.

(9) Legras, J. L., Erny, C., Le Jeune, C., Lollier, M., Adolphe, Y., Demuyter, C., Delobel, P., Blondin, B., and Karst, F. (2010) Activation of two different resistance mechanisms in Saccharomyces cerevisiae upon exposure to octanoic and decanoic acids. Appl. Environ. Microbiol. 76, 75267535.

(10) Liu, P., Chernyshov, A., Najdi, T., Fu, Y., Dickerson, J., Sandmeyer, S., and Jarboe, L. (2013) Membrane stress caused by octanoic acid in Saccharomyces cerevisiae. Appl. Microbiol.

Biotechnol. 97, 3239-3251. 


\subsection{High-throughput screening of an octanoic acid producer strain library enables detection of new targets for increasing titers in Saccharomyces cerevisiae}

Declaration of author contributions to the manuscript:

High-throughput screening of an octanoic acid producer strain library enables detection of new targets for increasing titers in Saccharomyces cerevisiae

Status: submitted November 2020, in revision

Type of manuscript: research article

Contributing authors: Leonie Baumann (LB), Stefan Bruder (SB), Johannes Kabisch (JK), Eckhard Boles (EB) \& Mislav Oreb (MO)

\section{Contributions of doctoral candidate and co-authors}

\section{(1) Concept and design}

Doctoral candidate LB: $80 \%$

Co-authors SB, JK, EB, MO: 5\%, 5\%, 5\%, 5\%

\section{(2) Conducting tests and experiments}

Doctoral candidate LB: $90 \%$, yeast molecular biology, biosensor measurements, FACS screening, fermentations, metabolite extraction and GC analysis, toxicity tests

Co-author SB: $10 \%$, FACS screening

\section{(3) Compilation of data sets and figures}

Doctoral candidate LB: $100 \%$, biosensor assays, FACS screening, metabolite titers and growth, toxicity test

\section{(4) Analysis and interpretation of data}

Doctoral candidate LB: $70 \%$, biosensor assays, FACS data, metabolite titers and growth, toxicity test

Co-authors SB, EB, MO: 10\%, 10\%, 10\%, FACS data, supervision and advice

(5) Drafting of manuscript

Doctoral candidate LB: $85 \%$

Co-authors SB, EB, MO: 5\%, 5\%, 5\% 


\title{
High-throughput screening of an octanoic acid producer strain library enables detection of new targets for increasing titers in Saccharomyces cerevisiae
}

\author{
Leonie Baumann, ${ }^{1}$ Stefan Bruder, ${ }^{2}$ Johannes Kabisch, ${ }^{2}$ Eckhard Boles ${ }^{1}$ and Mislav Oreb*1 \\ ${ }^{1}$ Institute of Molecular Biosciences, Faculty of Biological Sciences, Goethe University Frankfurt, \\ Max-von-Laue Straße 9, 60438 Frankfurt am Main, Germany \\ 2Department of Biology, Computer-aided Synthetic Biology, Technical University Darmstadt, \\ Schnittspahnstr. 10, 64287 Darmstadt, Germany \\ *Corresponding author: m.oreb@bio.uni-frankfurt.de
}

ABSTRACT: Octanoic acid is an industrially relevant compound with applications in antimicrobials or as a precursor for biofuels. Microbial biosynthesis through yeast is a promising alternative to current unsustainable production methods. To increase octanoic acid titers in Saccharomyces cerevisiae, we use a previously developed biosensor based on the octanoic acid responsive pPDR12 promotor coupled to GFP. We establish a biosensor strain amenable for high-throughput screening of an octanoic acid producer strain library. Through development, optimization and execution of a high-throughput screening approach, we were able to detect two new genetic targets, KCS1 and FSH2, which increased octanoic acid titers when overexpressed and have not been reported yet to be involved in fatty acid biosynthesis. The combined overexpression of both genes led to an increase in titers of $55 \%$ compared to the parental strain. The presented methodology can be employed to screen any genetic library and thereby more genes involved in improving octanoic acid production can be detected in the future.

KEYWORDS: Octanoic acid, C8 fatty acid, High-throughput Screening, overexpression library, FACS, yeast

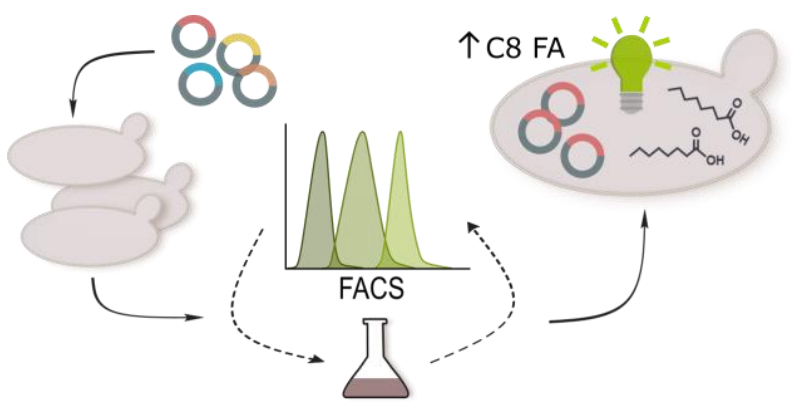




\section{INTRODUCTION}

Medium-chain fatty acids, such as octanoic acid (C8 fatty acid), are widely used in industry, for example as antimicrobials, or can be converted to fatty-acid derivatives like fatty alcohols, alkanes or alkenes, thereby widening the application range to biofuels, lubricants and surfactants. ${ }^{1}$ The current supply is mostly based on the extraction from oily plants, such as coconut and palm oil plants. However, these vegetable oils mostly consist of long-chain fatty acids with only minor fractions of octanoic and decanoic acid. ${ }^{2}$ Furthermore, the cultivation of oil plants oftentimes involves deforestation and extensive land use, $^{3}$ making other production methods from renewable substrates desirable. Engineering microbes like yeast to produce octanoic acid is a promising alternative with potentially less environmental impact.

In recent years, several reports have shown the successful engineering of $S$. cerevisiae to produce medium-chain (C6-C12) fatty acids. ${ }^{4-7}$ Most recently more than $1 \mathrm{~g} / \mathrm{L}$ medium-chain fatty acids were reported to be produced by an engineered S. cerevisiae strain. ${ }^{6}$ However, producing a mixture of chain lengths, reduces the yield of the desired fatty acid, and purification is difficult because different chain lengths have similar physicochemical properties. ${ }^{1}$ One way to achieve strains only producing specific fatty acid chain lengths, is by rational engineering of the fatty acid synthesis (FAS) genes. Gajewski et al. (2017) introduced an amino acid exchange of arginine to lysine (R1834K) in the MPT domain of FAS1, thereby generating an S. cerevisiae strain producing mainly octanoic acid. Apart from strategies for chain-length control, engineering efforts to generate higher titers have mostly focussed on enzymes involved in precursor routes for increasing cytosolic acetyl-CoA and malonyl-CoA supply or on decreasing fatty acid degradation. ${ }^{1,8,9}$ One example to achieve the latter, is knockout of $F A A 2$, encoding a medium-chain fatty acyl-CoA synthetase, which activates imported fatty acids in the peroxisomes for degradation via the $\beta$-oxidation pathway. ${ }^{10}$ Knockout of FAA2 has previously been demonstrated to increase short- and medium-chain fatty acid titers considerably. ${ }^{11,12}$

Nevertheless, to construct efficient cell factories, targeted engineering strategies are insufficient. Due to the complex cellular network, there are many factors whose direct or indirect involvement in fatty acid biosynthesis, transport, degradation or detoxification mechanisms are unknown so far. An alternative strategy to rational engineering of specific target genes to generate strains with higher product titers is the generation of strain libraries. However, strain evaluation is currently the rate limiting step in such efforts because traditional analytical methods like gas chromatography (GC) are very laborious and time-consuming. ${ }^{13,14}$ Biosensors in combination with a high-throughput detection technology, are a great tool to overcome this drawback. Biosensors 
enable easy and rapid detection of the desired compound, e.g. via fluorescence quantification. ${ }^{13,15-17}$ Previously, we have developed a whole-cell short-chain fatty acid biosensor amenable for high-throughput screenings. ${ }^{18}$ The biosensor is based on the short-chain fatty acid responsive promotor $p P D R 12$ coupled to GFP. PDR12 encodes an ATP-binding cassette transporter and its expression is regulated by the transcription factor War1p. ${ }^{19,20}$ War1p activates GFP expression via $P P D R 12$ regulation in the presence of inducing molecules, i. e. hexanoic, heptanoic and octanoic acid, in a concentration-dependent manner. For octanoic acid, the linear detection range was between $0.01-0.75 \mathrm{mM}(1.4-108 \mathrm{mg} / \mathrm{L})$ and it showed a high dynamic range with up to 10 -fold increase in signal after only $2 \mathrm{~h}$ of induction. ${ }^{18}$

Here, we modify the biosensor strain and verify its usability in flow cytometry. We aimed at detecting new targets that increase octanoic acid titers in S. cerevisiae through a high-throughput screening of a gene overexpression library via fluorescence-activated cell sorting (FACS). This approach enabled us to detect unexpected genes whose overexpression led to increased octanoic acid titers. To our knowledge, this is the first report of the application of an octanoic acid biosensor to identify genes enhancing octanoic acid levels in S. cerevisiae.

\section{RESULTS AND DISCUSSION}

Adaptation of an octanoic acid biosensor for use in a FACS-based screening. To adapt a previously developed octanoic acid biosensor ${ }^{18}$ for screening via FACS, we carried out several modifications. The original biosensor relied on a multi-copy plasmid carrying pPDR12-GFP. For a single cell screening through flow cytometry, we assumed that GFP expression from a multi-copy plasmid would create high single cell variation as a result of differing plasmid copy numbers per cell. This assumption was confirmed in a flow cytometry experiment (Figure S1). To avoid this effect, we considered two options: (1) Normalization of copy numbers through constitutive expression of another fluorescent gene (e. g. mCherry) on the same centromeric plasmid with the biosensor or (2) genomic integration of the biosensor. The first option has been shown to be successful for PHBA detection, ${ }^{21}$ however, it is more complex to execute because normalization of each measurement is inevitable. With the second option - a strain with genomically integrated biosensor - we had previously conducted measurements in a monochromator microplate reader. We had observed that the GFP signal was only barely distinguishable from a strain without sensor under octanoic acid supply. ${ }^{18}$ To verify the functionality of a genomically integrated biosensor in flow cytometry, we cultured strain LBY27 - containing the pPDR12-GFP cassette genomically - for $2 \mathrm{~h}$ with octanoic acid concentrations ranging between $0-80 \mathrm{mg} / \mathrm{L}$. After this induction period, the samples were screened employing flow cytometry and we observed an increase in fluorescence 
signal with increasing octanoic acid concentrations (Figure 1). Even though the fluorescence values partly overlapped for different concentrations, the mean fluorescence rose in correlation with the octanoic acid concentration. This verified that supplied octanoic acid is taken up by the cells and induces GFP production in a concentration-dependent manner. ${ }^{12,18}$ Furthermore, we asserted that the detection in the flow cytometer is much more sensitive than in the microplate reader confirming that the genomically integrated biosensor is generally suitable for the envisioned screening.

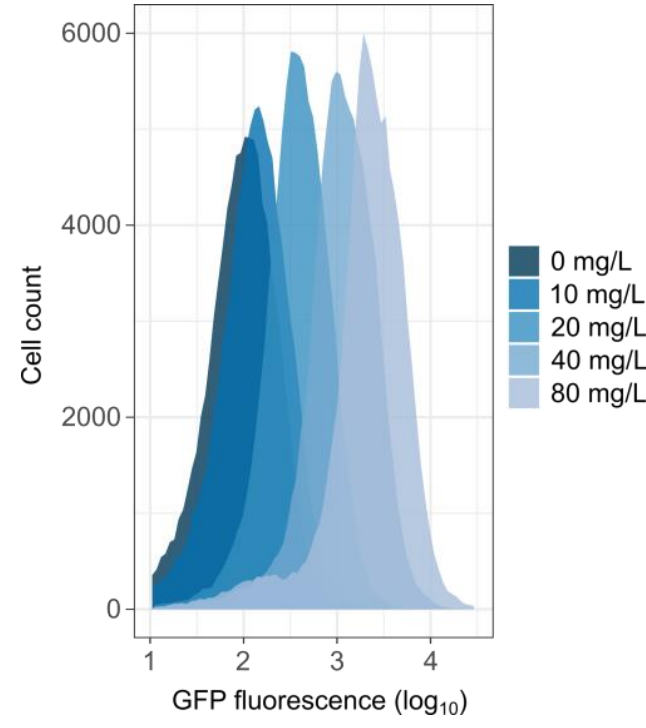

Figure 1: Evaluation of biosensor response to supplementation with octanoic acid. Strain LBY27, which contains a stable genomic integration of the biosensor pPDR12-GFP, was inoculated to an $\mathrm{OD}_{600}=$ 0.2 and grown in SCD medium for $3 \mathrm{~h}$. The indicated octanoic acid amounts were added and the cultures were grown for another $2 \mathrm{~h}$ before measurement of GFP fluorescence in a flow cytometer (common logarithmic transformation of fluorescence area plotted). Results are shown for a single experiment; the experiment was repeated with comparable results (Figure S2).

In the next step, we genomically integrated the biosensor in an octanoic acid producer strain. To enable octanoic acid production, the strain contains a mutation in the FAS1 gene at position 1834 bp, resulting in an amino acid exchange from arginine to lysine $\left(F A S 1^{R K}\right) .{ }^{4}$ This strain, LBY31, furthermore contains a positive-feedback loop ${ }^{21}$ to increase the dynamic range of the sensor. For the positive-feedback loop, the promotor of the transcriptional regulator-encoding gene WAR1 was replaced with its target promotor PPDR12. This was shown to increase the dynamic range of GFP expression in response to para-hydroxybenzoic acid (PHBA), another activator of pPDR12. ${ }^{21}$

When growing LBY31 in buffered SCD medium, we observed that the $35 \mathrm{mg} / \mathrm{L}$ octanoic acid produced $24 \mathrm{~h}$ after inoculation were reduced to about $50 \%$ after $46 \mathrm{~h}$. After $72 \mathrm{~h}$ of fermentation, almost no octanoic acid was detectable anymore (Figure 2). To prevent octanoic acid degradation, we deleted $F A A 2$, encoding a fatty acyl-CoA synthetase involved in the activation of free mediumchain fatty acids prior to $\beta$-oxidation. ${ }^{10}$ It has previously been shown that deletion of FAA2 prevents the degradation of medium-chain fatty acids such as octanoic acid. ${ }^{11,12}$ The resulting strain LBY39 indeed maintained the octanoic acid titer at the same level even $72 \mathrm{~h}$ after 
inoculation. Therefore, the knockout of FAA2 proved to be essential to maintain octanoic acid titers stable over several days of fermentation. Stable titers are important to prevent false-positive strains in the screening, which might have varying titers due to varying degrees of intracellular octanoic acid degradation. The resulting biosensor strain LBY39 has a high linear and dynamic range of detection and produced octanoic acid amounts within the detection range of the sensor ( $35 \mathrm{mg} / \mathrm{L})$.

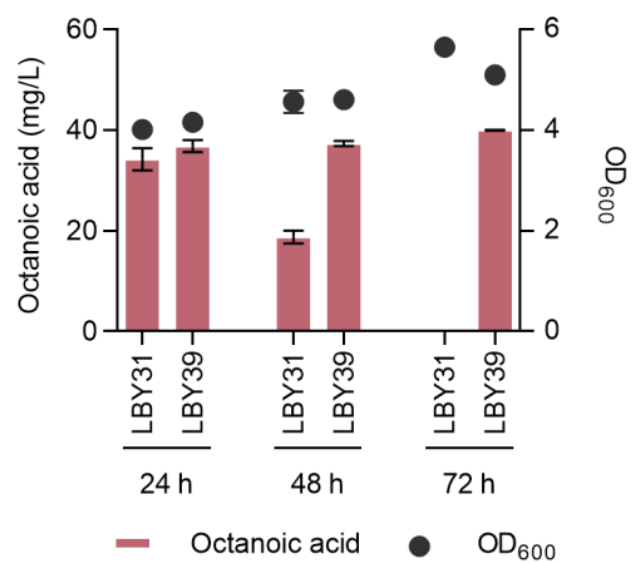

Figure 2: Effect of FAA2 knockout on octanoic acid titers in biosensor strain. $\mathrm{OD}_{600}$ (dots) and octanoic acid titers (bars) of LBY31 and LBY39 fermented in buffered SCD medium over $72 \mathrm{~h}$. LBY31 (producer): pPDR12-GFP, FAS1 ${ }^{R K}$. LBY39 (producer): pPDR12-GFP, FAS1 ${ }^{R K}$, $\triangle$ faa2.Fatty acids were extracted, methylated and quantified via GC. $n=2$, error bars $= \pm$ SD.

We then sought to test whether the octanoic acid producer strain LBY39 can be distinguished from the non-producer LBY27 in flow cytometer analysis which we performed $4 \mathrm{~h}, 24 \mathrm{~h}$ and $48 \mathrm{~h}$ after inoculation (Figure 3). After only $4 \mathrm{~h}$, LBY39 showed an increase in the mean fluorescence compared to the non-producer. Since octanoic acid production mainly occurs after glucose depletion (approx. $18 \mathrm{~h}$ ), this difference was much more pronounced after $24 \mathrm{~h}$. After $48 \mathrm{~h}$, producer and non-producer populations become clearly distinct with median values differing by a factor of about $10^{2}$. This suggests that the higher intracellular octanoic acid levels in LBY39 after $48 \mathrm{~h}$ also lead to higher GFP expression - despite loss of octanoic acid to the extracellular environment. 

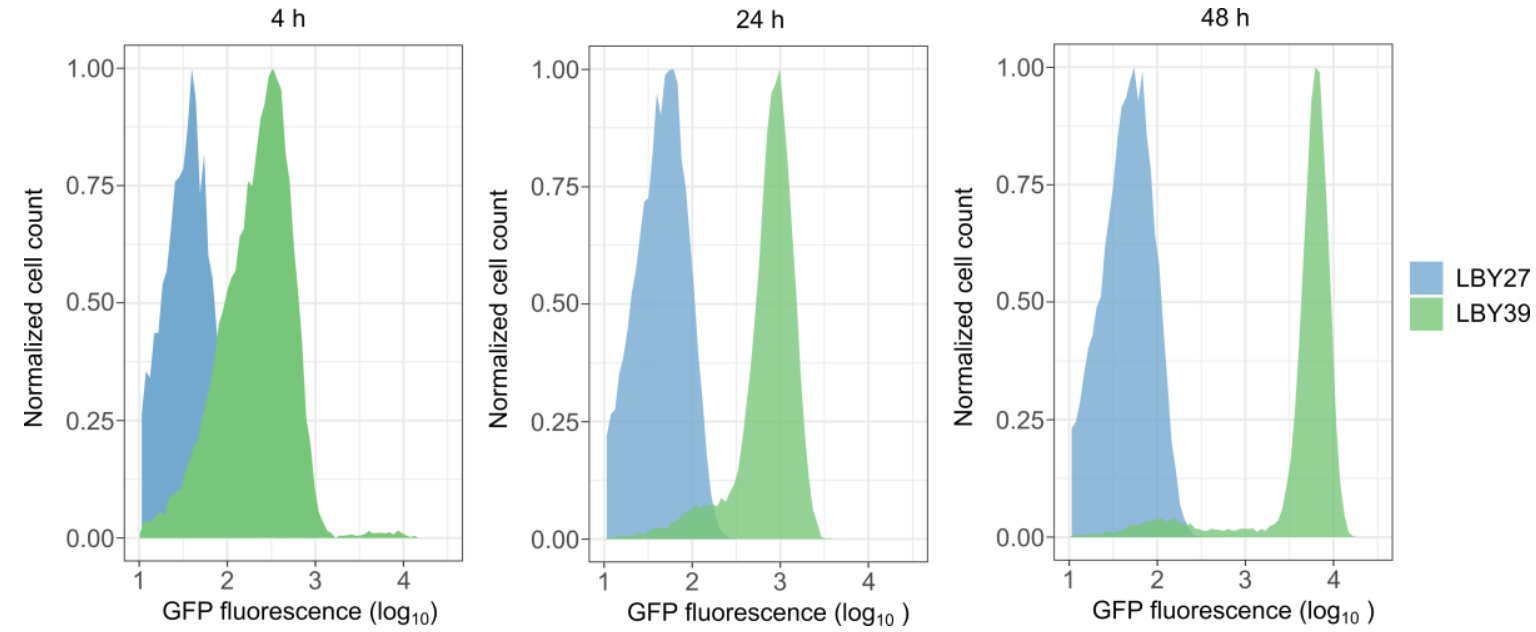

Figure 3: Fluorescence signal of the genomic octanoic acid sensor in producer and non-producer strain. Strains LBY27 and LBY39 contain a stable integration of the biosensor $p$ PDR12-GFP. Strains were inoculated to $\mathrm{OD}_{600}=0.1$ and grown in buffered SCD medium. Aliquots were taken after $4 \mathrm{~h}, 24 \mathrm{~h}$ and $48 \mathrm{~h}$ for GFP fluorescence measurement in a flow cytometer (common logarithmic transformation of fluorescence area plotted). LBY27 (non-producer). LBY39 (producer): FAS1 ${ }^{R K}, \triangle$ faa2.

High-throughput screening for genes enhancing octanoic acid levels. Our general procedure for a high-throughput screening is depicted in Figure 4. To find genes that when overexpressed lead to an increase in octanoic acid titers, we combined the sensor strain LBY39 with a gene overexpression library. Such libraries allow for detection of genes that when overexpressed (through the presence of high plasmid copy numbers) will have a positive effect on the titer of the produced compound. ${ }^{22-24}$ We used a library ${ }^{25}$ containing approximately $10 \mathrm{~kb}$ fragments of the entire yeast genome cloned on multi-copy $(2 \mu)$ vectors with each vector comprising roughly 3-10 ORFs flanked by endogenous up- and downstream sequences. To prepare the library from E. coli glycerol stocks, we grew the 1588 strains individually in 96-deepwell plates ensuring thereby representation of all plasmids. Through transformation of LBY39 with a mixture of all library plasmids, about 22,000 colonies were generated - resulting in a theoretical coverage of the library of almost 14 times, assuming equal transformation probability of all plasmids. We inoculated eight shake flasks with colonies from 3-4 transformation plates each and grew them in buffered SCD medium $(\mathrm{pH}=6.5)$. At $\mathrm{pH} 6.5$, octanoic acid is mostly present in its dissociated form and hence unable to cross the plasma membrane of the cell for re-entry after secretion. By choosing this $\mathrm{pH}$, we aimed at minimizing octanoic acid uptake from the medium, thereby avoiding cross-exchange of octanoic acid between cells.

The sampling was performed $48 \mathrm{~h}$ after inoculation to favour enrichment of cells with high octanoic acid titers and simultaneously good growth (Figure S3). Our high-throughput screening design was based on the attempt to decrease false positive results by integrating three 
subsequent enrichment steps (E1-E3). Iterative screening rounds are essential to account for the variation of production on a single cell level. To avoid too strict cut-offs, we slowly decreased the percentage of highest fluorescent events sorted from E1 to E3. In the first round, we collected the highest $10 \%$ of fluorescent events into fresh liquid medium from each of the 8 flasks separately (E1) and let them grow for $66 \mathrm{~h}$. After this time period, we conducted another flow cytometry measurement. Here, the populations from flasks 1-6 showed a low fluorescence close to the negative control strain LBY27 (Figure S4). We therefore solely continued with flasks 7 and 8 . To lower the rate of false positives, we conducted two subsequent cycles of enrichment after the first sorting, collecting the top $7.5 \%$ fluorescent events (E2) and the top $5 \%$ and top $1 \%$ (E3), respectively. LBY39 containing the empty vector was grown and measured in the flow cytometer as a control. Fluorescence signals of the sorting steps are shown in Figure S3, S4 and S5. The populations from flasks 7 and 8 from E2 and E3 showed a lower mean fluorescence than the control strain LBY39 containing the empty vector (Figure S4, S5). The control strain, however, was always inoculated freshly to an $O D=0.1$ and not sorted like the populations from flasks 7 and 8 . Therefore, the fluorescence signal might have varied due to growth-dependent octanoic acid amounts.

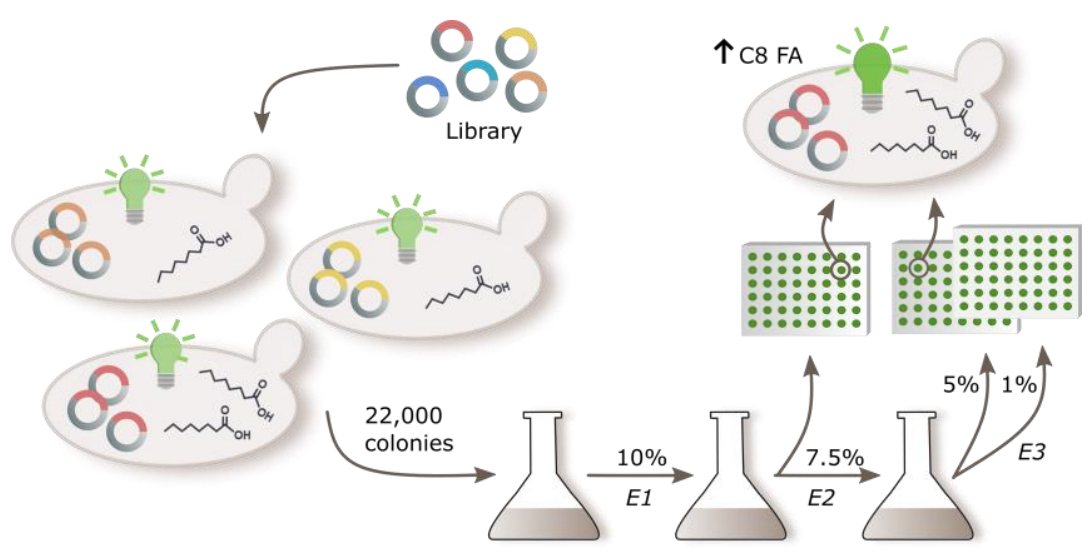

Figure 4: High-throughput screening of an octanoic acid producer strain library. The producer and biosensor strain LBY39 was transformed with a multi-copy yeast genomic library resulting in roughly 22,000 colonies. These were analysed in three consequent enrichment steps (E1-E3) and highest fluorescent events were sorted via FACS. Random colonies of enriched cultures were analysed further and strains that increase octanoic acid (C8 FA) titers can be detected.

Identification and verification of candidate plasmids. Next, we analysed the colonies of the enriched libraries. From the sorted events, about $80 \%$ resulted in colony growth (approx. 200 per plate). To extract library plasmids from these colonies, we randomly picked about 10 colonies per plate, inoculated them, extracted plasmids and re-transformed them into E. coli for plasmid amplification. Not all transformations led to E. coli colony growth. Nevertheless, we were able to successfully extract six plasmids from flask 7 of E2 and one plasmid of E3. From flask 8, we 
extracted eight plasmids of E2 and two plasmids of E3. To identify genes, we sequenced the ORFs on the plasmids and ran BLAST searches. Thereby, we successfully assigned all plasmids to the library (Table 1).

Table 1: Identification of library plasmids after high-throughput screening. Single colonies were analysed from flask 7 and $8(F 7, F 8)$ after different enrichment rounds (E1-E3) from top fluorescent sorted events (top $7.5 \%, 5 \%, 1 \%)$. Plasmids from these cells were extracted and the contained genes identified via BLAST search.

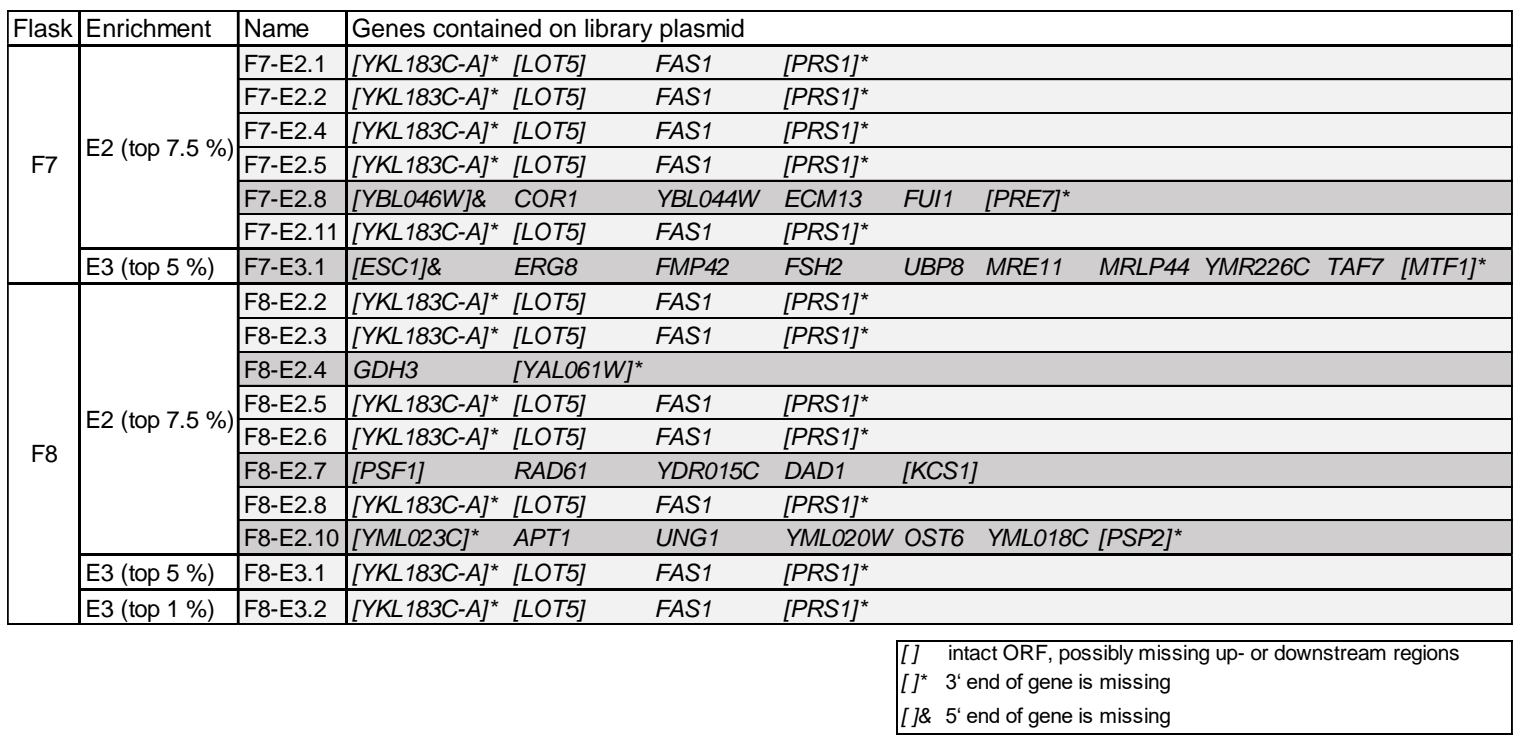

Of the 17 plasmids we identified, 12 corresponded to a library plasmid containing two complete ORFs (LOT5, FAS1) as well as two truncated ORFs (YKL183C-A, PRS1). It was striking that the library plasmid containing the wild type variant of FAS1 was enriched in the screening after E2 and E3. We assumed that this might be the result of improved growth conferred through wild type FAS1. To evaluate this hypothesis, we transformed producer strain LBY38 with this library plasmid containing wild type FAS1, the library plasmid containing wild type FAS2, or the control vector pGP564, respectively. $48 \mathrm{~h}$ after inoculation, we extracted fatty acids and quantified via GC measurement. Indeed, we found that the FAS1-library plasmid led to slightly decreased octanoic acid titers but improved growth and, interestingly, higher C6, C10 and C12 fatty acid levels, whereas the library plasmid containing FAS2 did not have an effect on either of these factors (Figure 5A, B). To confirm that this is the result of the FAS genes and not any of the other genes encoded on the library plasmids, we transformed LBY38 with plasmids solely containing FAS1, FAS2 or FAS1 ${ }^{R K}$ or different combinations thereof. We were able to confirm that expression of wild type FAS1 in LBY38 decreases octanoic acid titers (Figure S6). ${ }^{26}$ Thereby, probably toxic effects on the cell are reduced. This suggests, that FAS1 gives the cells an advantage over other cells and therefore appears enriched after iterative screening rounds. The observation that strains containing the FAS1 library plasmid still have fluorescence in the range of the top fluorescing cells 
of the population might result from increased production of $\mathrm{C} 6$ and $\mathrm{C} 10$ fatty acids. C6 fatty acid also strongly activates the biosensor but is less toxic than C8 fatty acid and for C10 fatty acid an activation of $p P D R 12$ could not be excluded in previous experiments. ${ }^{18}$

A)

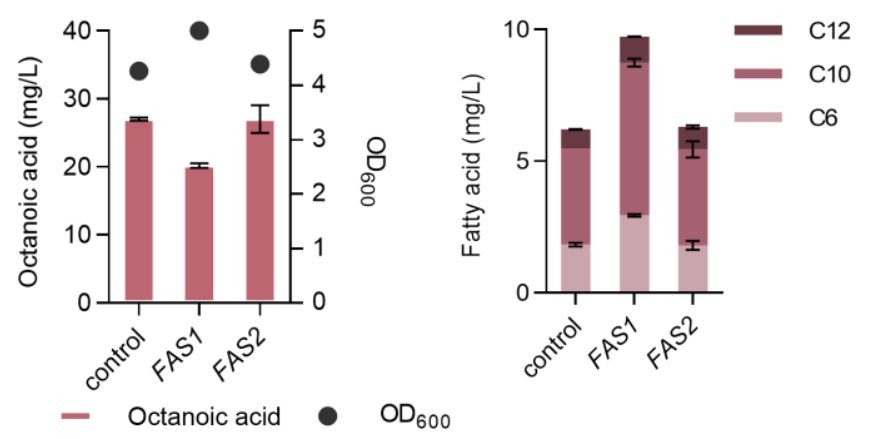

C)

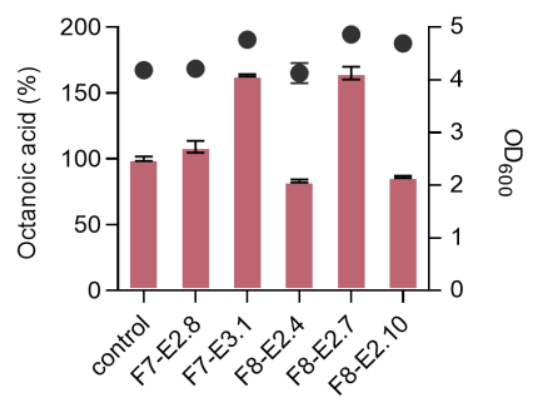

Figure 5: Effect of identified library plasmids on fatty acid titers and growth. $\mathrm{OD}_{600}$ (dots) and octanoic acid titers (bars) (A, C) and C6, C10 and C12 fatty acid titers (B) of octanoic acid producer strain LBY38 containing either the empty vector pGP564 (control) or library plasmids with wild type FAS1 or FAS2, respectively, or other library plasmids as indicated. Fatty acids were extracted, methylated and quantified via GC $48 \mathrm{~h}$ after inoculation in buffered SCD medium. LBY38 (producer): $F A S 1^{R K}, \triangle f a a 2 . n=2$, error bars $= \pm S D$.

Apart from the enriched FAS1 library plasmid, we identified five more plasmids. Even though none of these plasmids was enriched, we assumed that they could improve growth and/or octanoic acid titers being present after at least one enrichment round. We transformed the producer LBY38 with each of the five plasmids and quantified produced octanoic acid $48 \mathrm{~h}$ after inoculation. Two of the plasmids, namely F7-E3.1 (Elask 7, Enrichment 3) and F8-E2.7 (Elask 8, Enrichment 2), led to an increase in octanoic acid titers as well as improved growth (Figure 5C).

Identification and verification of candidate genes. As both plasmids, F7-E3.1 and F8-E2.7, contain several genes (Table 1), we were seeking to find the one(s) responsible for the positive effect. For this purpose, we re-cloned the genes and repeated fermentations (Figure S7, Figure 6). Thereby, we identified $F S H 2$, putatively encoding a serine hydrolase, ${ }^{27}$ and $K C S 1$, encoding an inositol hexa-/heptakisphosphate kinase, ${ }^{28,29}$ as the genes responsible for the increase in growth and octanoic acid titer (Figure 6A, B). When overexpressing both FSH2 and KCS1 from one multicopy plasmid, we observed an increase in octanoic acid titer of $55 \%$ as well as higher OD (Figure $6 C)$.

The biological role of $F S H 2$ is unknown. A large-scale study showed that Fsh1p localized to the cytoso $^{30}$ and in a study combining computational and experimental proteomics, it was assigned to a new group of serine hydrolases together with $F S H 1$ and $F S H 3 .{ }^{27}$ Overexpression of $F S H 1$ was reported to decrease phospholipids and increase triacylglycerols, lipid droplets and free fatty 
acids. ${ }^{31}$ As FSH1 and $F S H 2$ share $21-26 \%$ homology, ${ }^{31}$ it seems likely that $F S H 2$ also plays a role in lipid metabolism and thereby leads to increased octanoic acid titers when overexpressed - but due to a lack of knowledge about $F S H 2$, this is speculative. The molecular and biological function of $K C S 1$, is well characterized. It encodes an inositol pyrophosphate synthase which phosphorylates a variety of inositol phosphate substrates. ${ }^{28,29,32}$ Inositol pyrophosphates serve as signalling molecules in many cellular processes. KCS1 was shown to play a role in vesicular trafficking, cell wall integrity and stress response. ${ }^{29,33}$ Deletion of KCS1 resulted in strong phenotypes with increased cell size and impaired growth. The exact link of how KCS1 overexpression leads to an increase in octanoic acid titers remains to be investigated. Suspecting that the detected genes might influence robustness towards octanoic acid, we transformed nonproducer CEN.PK2-1C with plasmids containing FSH2, KCS1 and both, respectively, and supplied octanoic acid in concentrations from 0-300 mg/L. However, both FSH2 and KCS1 did not increase robustness towards octanoic acid (Figure 6D).

A)

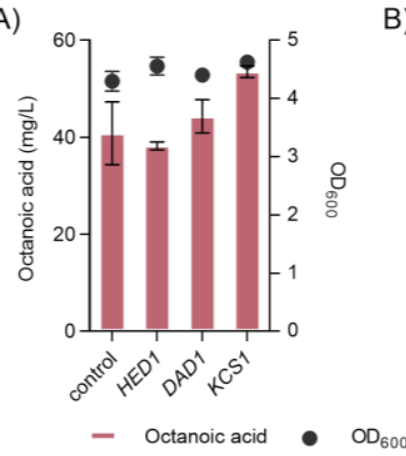

B)

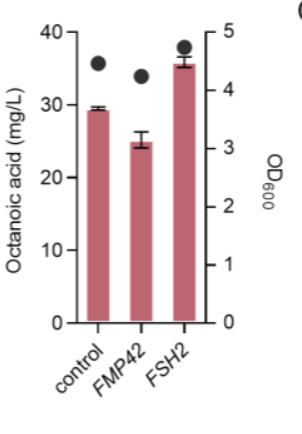

C)

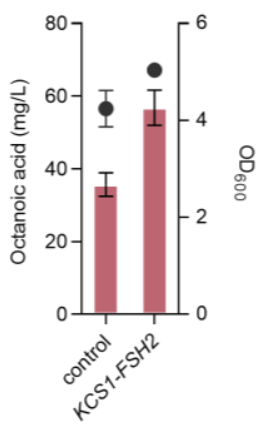

D)

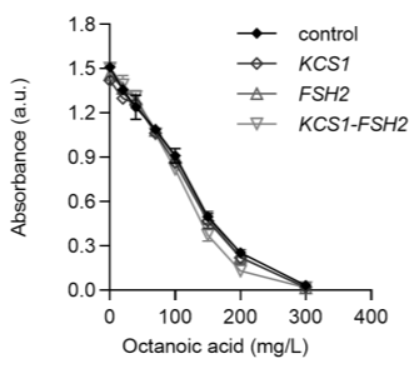

Figure 6: Identification of genes increasing octanoic acid titers and strain growth but not resistance. $(A-C)$ $\mathrm{OD}_{600}$ (dots) and octanoic acid titers (bars) of octanoic acid producer strain LBY38 containing either the empty vector pGP564 (control) or plasmids with indicated genes. Fatty acids were extracted, methylated and quantified via GC $48 \mathrm{~h}$ after inoculation in buffered SCD medium. LBY38 (producer): FAS1 ${ }^{R K}, \Delta$ faa $2 . n=$ 2 , error bars $= \pm S D$ (D) Non-producer CEN.PK2-1C containing either the empty vector pGP564 (control) or plasmids with the indicated genes was inoculated to $O D=0.05$ and supplied with $0-300 \mathrm{mg} / \mathrm{L} \mathrm{octanoic} \mathrm{acid.}$ Growth was measured by absorbance after 20 h. $n=3$, error bars $= \pm S D$

We demonstrated the utility of employing a biosensor in a high-throughput screening via FACS to detect new targets that increase octanoic acid titers. This screening shows how useful the development of biosensors for industrially relevant compounds is. The developed method is very versatile and could also be employed with other gene libraries to detect even more new targets. This high-throughput screening approach enabled us to detect two entirely new genetic targets, KCS1 and FSH2, which could not have been detected in a rational engineering approach. This is 
the first report that $K C S 1$ and $F S H 2$ play a role in fatty acid biosynthesis and which, in combined overexpression, increased octanoic acid titers by $55 \%$ compared to the parental strain.

\section{METHODS}

Strains and Plasmid Construction. Yeast strains used throughout this study are listed in Table 2, and plasmids and oligonucleotides in Supplementary Table S1 and S2, respectively.. For genomic integration of the biosensor ( $\triangle$ ura3::pPDR12-EnvyGFP-tCYC1) into LBY31, CRISPR/Cas9 was used as described previously. ${ }^{34}$ The CRISPR/Cas9 plasmid was amplified in two PCR fragments, assembled in vitro in an isothermal reaction using T5 exonuclease, polymerase and ligase ${ }^{35}$ and transformed into E. coli. After verification via Sanger sequencing, it was transformed into the target strain together with the respective insertion fragment. The same approach was used for: integration of the positive feedback loop ( $\triangle p W A R 1:: p P D R 12)$ into LBY31 and $\triangle$ faa 2 knockout in LBY39. The insertion fragments were amplified with overhangs from the following template DNAs: biosensor from LBY27 genomic DNA; positive feedback loop from LBV14 plasmid DNA; $\triangle$ faa2 donor DNA from $\mathrm{SHY} 34^{26}$ genomic DNA.

Table 2: Yeast strains.

\begin{tabular}{|c|c|c|}
\hline Strain & Genotype & Reference \\
\hline CEN.PK2-1C & $\begin{array}{l}\text { MATa; MAL2-8c; SUC2 ; ura3-52; } \\
\text { his3 } 31 ; \text { leu2-3_112; trp1-289 }\end{array}$ & $\begin{array}{l}\text { Euroscarf, Frankfurt } \\
\text { am Main, Germany }\end{array}$ \\
\hline VGY2 & $\begin{array}{l}\text { CEN.PK2-1c } \triangle p F A S 1-1-300:: p H X T 7-1 \\
392 \triangle p F A S 2-1-200:: p H X T 7-1-392 \\
\text { FAS1 }\end{array}$ & $\begin{array}{l}\text { Wernig et al., } 2021 \\
\text { (submitted) }\end{array}$ \\
\hline LBY27 & $\begin{array}{l}\text { CEN.PK113-11C } \triangle p y k 2:: p P D R 12- \\
\text { EnvyGFP-tCYC1 }\end{array}$ & Baumann et al., 2018 \\
\hline LBY31 & $\begin{array}{l}\text { VGY2 } \triangle \text { ura3 ::pPDR12- EnvyGFP- } \\
\text { tCYC1 } \triangle p W A R 1:: p P D R 12\end{array}$ & This study \\
\hline LBY38 & VGY2 $\Delta$ faa2 & $\begin{array}{l}\text { Wernig et al., } 2021 \\
\text { (submitted) }\end{array}$ \\
\hline LBY39 & LBY31 $\Delta$ faa2 & This study \\
\hline
\end{tabular}

For cloning plasmids with one or more genes (LBV91-113), the desired fragments were amplified by PCR from the original library plasmids with overhangs to the sequences flanking the BamHI/PstI cut sites of pGP564. pGP564 was digested with BamHI/Pstl and transformed together with the insertion fragments into CEN.PK2-1C and plasmids were assembled via homologous recombination in yeast. Yeast transformations were performed according to Gietz and Schiestl. ${ }^{36}$ Cells were streaked out on selective SCD medium ${ }^{37}$ lacking leucine (SCD-leu) to select for $L E U 2$. The colonies from the yeast plates were collectively transformed into electrocompetent $E$. coli DH10 (Gibco BRL, Gaithersburg, MD) and transformants were selected on lysogeny broth (LB) agar 
plates $^{38}$ supplemented with kanamycin $\left(50 \mu \mathrm{g} \mathrm{mL}^{-1}\right)$. Selected plasmids were extracted according to standard procedures and verified via Sanger sequencing.

Preparation of library plasmids for screening. The library was present in 1796 -well E. coli glycerol stocks. To ensure that all plasmids were represented, we inoculated the $E$. coli strains in $1 \mathrm{ml}$ of LB $^{\text {Kan }}$ in 17 96-deepwell plates (Flat-bottom blocks, Qiagen) and grew them overnight under shaking (250 r.p.m.). The cultures of each deepwell plate were mixed and plasmids extracted via 17 midi-preps (GeneJET ${ }^{\mathrm{TM}}$ Plasmid-Midiprep-Kit, Thermo Fisher Scientific). For later use, a mixture of all plasmid extracts was prepared.

Cultivation and flow cytometry analysis. For pre-cultures, several colonies of respective strains were inoculated in (selective) SCD with $100 \mathrm{mM}$ potassium phosphate buffer adjusted to $\mathrm{pH} 6.5$ and shaken (180 r.p.m.) at $30{ }^{\circ} \mathrm{C}$ overnight. The main cultures were inoculated (in replicates as indicated for each figure) to an $\mathrm{OD}_{600}$ of 0.1 in buffered SCD medium in shake flasks and grown for up to $72 \mathrm{~h}$ with shaking $\left(180\right.$ r.p.m., $30^{\circ} \mathrm{C}$ ). Aliquots were taken for flow cytometry measurements and analyses were performed using a Sony SH800SA with a $488 \mathrm{~nm}$ argon laser and a $100 \mu \mathrm{m}$ sorting chip. Instrument gains were set as follows: Forward scatter values were set to 6 , thresholds for backscatter and FL2 channel ("GFP signal": $525 \pm 50 \mathrm{~nm}$ ) to $43 \%$ and $30 \%$, respectively. Sample pressure was set to a maximum of 6 , to keep the event per second rate below 10,000 events. For each analysis, $10^{5}$ events were evaluated. For cell sorting, cell agglomerates (doublets) were excluded based on channels FSC-H/FSC-W, BSC-H/BSC-W and FSC-A/BSC-A.

High-throughput screening via FACS. For transformation of LBY39 with the library, a mixture of the 17 plasmid extracts was used. About 22,000 colonies resulted from the transformation and we inoculated eight shake flasks with colonies from 3-4 transformation plates each. These were grown in buffered SCD ${ }^{\text {-leu }}$ medium $(\mathrm{pH}=6.5$ ) alongside control strains (LBY27, LBY39+pGP564) in shake flasks (180 r.p.m., $30^{\circ} \mathrm{C}$ ). Aliquots were taken after $48 \mathrm{~h}$ (Enrichment 1 ) and measured via flow cytometry as described above. A gate was set to sort the $10 \%$ events with highest fluorescence ( 300,000 events, mode "purity") into $15 \mathrm{ml}$ falcons containing fresh media. For all 8 flasks, sorting was performed, and samples were grown again for $66 \mathrm{~h}$ in shake flasks (approximated starting $\mathrm{OD}_{600} \sim 0.0025$ ). After $66 \mathrm{~h}$, another round of sorting (Enrichment 2) was performed for flask 7 and 8 . The $7.5 \%$ highest fluorescent events were sorted into falcons as well as onto $\mathrm{SCD}^{\text {-leu }}$ solid medium (384 events). This procedure was repeated after another incubation period of $66 \mathrm{~h}$ in shake flasks, this time sorting only onto solid $\mathrm{SCD}^{\text {-leu }}$ of the top $5 \%$ and $1 \%$ fluorescent events, respectively (Enrichment 3). 
Identification and verification of plasmids after screening. From the sorted plates resulting from the screening, we randomly picked about 10 colonies per sorted plate for growth in SCD-leu. Plasmids were extracted by standard procedures with an additional step of disrupting the yeast cells with glass beads ( $\varnothing 0.25-0.5 \mathrm{~mm}$, Roth, Karlsruhe, Germany) by vigorous shaking in lysis buffer for $8 \mathrm{~min}$. For amplification, the plasmids were transformed in electrocompetent $E$. coli DH10 $\beta$ (Gibco BRL, Gaithersburg, MD). Plasmids were extracted from E. coli by standard procedures and sequenced with Sanger sequencing with primers LBP85, LBP237 or LBP238. Identification of library plasmids was performed via BLAST searching the Saccharomyces cerevisiae Genome Database (http:// www.yeastgenome.org/).

Cultures for Fatty Acid Production. S. cerevisiae strains were grown as previously described ${ }^{4}$ with some adjustments. For pre-cultures, several colonies of a strain were inoculated in (selective) SCD with $100 \mathrm{mM}$ potassium phosphate buffer adjusted to pH 6.5 and grown overnight (180 r.p.m., 30 ${ }^{\circ} \mathrm{C}$ ). Main cultures were inoculated to an $\mathrm{OD}_{600}$ of $0.1 \mathrm{in} 50 \mathrm{ml}$ of the respective medium and incubated in $300 \mathrm{~mL}$ shake flasks under the same conditions. For sampling, cultures were harvested by centrifugation and $10 \mathrm{~mL}$ of the supernatant was used for fatty acid extraction.

Fatty Acid Extraction and Derivatization. Fatty acid extraction and derivatization were performed as described previously. ${ }^{12}$ Cells were separated from the medium ( $3500 \mathrm{rcf}, 10 \mathrm{~min}$ ), an internal standard ( $0.2 \mathrm{mg}$ heptanoic acid) was added to $10 \mathrm{~mL}$ supernatant and mixed with $1 \mathrm{~mL} 1 \mathrm{M} \mathrm{HCl}$ and $2.5 \mathrm{~mL}$ methanol/chloroform solution (1:1). After vigorous shaking for $3 \mathrm{~min}$, the mixture was centrifuged at $3000 \mathrm{rcf}$ for $10 \mathrm{~min}$ and the chloroform layer was recovered and evaporated overnight. The methylation of the fatty acids was performed as previously described. ${ }^{39}$ Samples were dissolved in $200 \mu \mathrm{L}$ toluene, mixed with $1.5 \mathrm{~mL}$ methanol and $300 \mu \mathrm{L} 8.0 \%(\mathrm{w} / \mathrm{v}) \mathrm{HCl}$ solution (diluted in methanol), vortexed, and incubated at $100{ }^{\circ} \mathrm{C}$ for $3 \mathrm{~h}$ to form fatty acid methyl esters (FAME). After cooling at $4{ }^{\circ} \mathrm{C}$ for $10 \mathrm{~min}, 1 \mathrm{~mL} \mathrm{H} \mathrm{H}_{2} \mathrm{O}$ and $1 \mathrm{~mL}$ hexane were added to the sample, followed by thorough shaking, and the organic phase was transferred to a GC vial.

GC-FID analysis of FAMEs. GC analyses were carried out on a Perkin Elmer Clarus 400 instrument (Perkin Elmer, Germany) equipped with an Elite FFAP capillary column (30 m $\times 0.25 \mathrm{~mm}$, film thickness: $0.25 \mu \mathrm{m}$; PerkinElmer, Germany) and a flame ionization detector (Perkin Elmer, Germany) as described previously. ${ }^{12}$

Toxicity test. CEN.PK2-1C was transformed with plasmids pGP564, LBV106, LBV112 and LBV113, respectively, and plated on SCD ${ }^{\text {-leu}}$. Pre-cultures were inoculated in triplicates in buffered SCD-leu and grown over night with shaking ( $30^{\circ} \mathrm{C}, 180$ r.p.m.). For main cultures, strains were inoculated to an $\mathrm{OD}_{600}$ of 0.2 and incubated for about 5 hours. The cultures were then diluted in fresh media 
to an $\mathrm{OD}_{600}$ of 0.05 and transferred into a 96 -well plate (clear with flat bottom, greiner bio-one) with $50 \mathrm{\mu l} /$ well. A dilution series was made with octanoic acid (Sigma-Aldrich, GC grade) diluted in the same media, to reach final concentrations in the wells of $0-300 \mathrm{mg} / \mathrm{L}$ when $200 \mu \mathrm{l}$ of the respective dilutions was added to the strains per well. All three replicates of each strain were inoculated in technical triplicates in the well plates. The starting absorbance was measured in a platereader (CLARIOstar ${ }^{\circledR}$, BMG Labtech, Ortenberg, Germany) and plates were incubated for about $20 \mathrm{~h}$ at $30{ }^{\circ} \mathrm{C}$ without shaking before absorbance was measured again. From final absorbance values, a blank value (media without strain) was subtracted.

Software. Flow cytometry data evaluations and graphical presentations were performed with $\mathrm{R}$ v3.6.3 using packages readr v1.3.1, plyr v1.8.6 and tidyverse v1.3.0. Data tables were stored in Microsoft Excel 2016. Other graphs were made using the software Prism 9 (GraphPad, USA).

Abbreviations. E1-E3: Enrichment 1-3; FACS: fluorescence-activated cell sorting; FAS: fatty acid synthase; GC: gas chromatography; MPT: malonyl-palmitoyl transferase; $\mathrm{OD}_{600}$ : optical density at $\lambda=600 \mathrm{~nm}$; ORF: open reading frame; SD: standard deviation

\section{ASSOCIATED CONTENT}

\section{Supporting Information}

Table S1. Table of strains and plasmids. Table S2. Table of oligonucleotides. Figure S1. Genomically integrated versus plasmid-based biosensor in flow cytometry. Figure S2. Biosensor response to supplemented octanoic acid. Figure S3. Fluorescence signal of library population, E1 sorting. Figure S4. Fluorescence signal of library population, E2 sorting. Figure S5. Fluorescence signal of library population, E3 sorting. Figure S6. Expression of FAS genes in LBY38. Figure S7. Identification of genes increasing octanoic acid titers and growth

\section{AUTHOR INFORMATION}

\section{Corresponding Author}

*E-mail: m.oreb@bio.uni-frankfurt.de

\section{Author Contributions}

LB and MO conceived the study. LB performed all experiments and wrote the paper. SB assisted with FACS experiments and data analysis. JK and EB provided advice and resources. All authors read, reviewed and approved the manuscript. 


\section{Notes}

EB is inventor of EP patent application No. 15162192.7 filed on April 1, 2015, and of EP patent application No. 15174342.4 filed on June 26, 2015, by Goethe-University Frankfurt, concerning short-chain acyl-CoA producing FAS variants. There are no other competing interests.

\section{ACKNOWLEDGMENTS}

We thank Christine Essl for experimental support. This project has received funding from the European Union's Horizon 2020 research and innovation programme under grant agreement No. 720824.

\section{REFERENCES}

(1) Sarria, S., Kruyer, N. S., and Peralta-Yahya, P. (2017) Microbial synthesis of medium-chain chemicals from renewables. Nat. Biotechnol. 35, 1158-1166.

(2) Yan, Q., and Pfleger, B. F. (2020) Revisiting metabolic engineering strategies for microbial synthesis of oleochemicals. Metab. Eng. 58, 35-46.

(3) Schmidt, J. H. (2015) Life cycle assessment of five vegetable oils. J. Clean. Prod. 87, 130-138.

(4) Gajewski, J., Pavlovic, R., Fischer, M., Boles, E., and Grininger, M. (2017) Engineering fungal de novo fatty acid synthesis for short chain fatty acid production. Nat. Commun. 8, 14650.

(5) Leber, C., Polson, B., Fernandez-Moya, R., and Da Silva, N. A. (2015) Overproduction and secretion of free fatty acids through disrupted neutral lipid recycle in Saccharomyces cerevisiae. Metab. Eng. 28, 54-62.

(6) Zhu, Z., Hu, Y., Teixeira, P. G., Pereira, R., Chen, Y., Siewers, V., and Nielsen, J. (2020) Multidimensional engineering of Saccharomyces cerevisiae for efficient synthesis of mediumchain fatty acids. Nat. Catal. 3, 64-74.

(7) Zhu, Z., Zhou, Y. J., Krivoruchko, A., Grininger, M., Zhao, Z. K., and Nielsen, J. (2017) Expanding the product portfolio of fungal type I fatty acid synthases. Nat. Chem. Biol. 13, 360-362.

(8) Fernandez-Moya, R., and Da Silva, N. A. (2017) Engineering Saccharomyces cerevisiae for highlevel synthesis of fatty acids and derived products. FEMS Yeast Res. 17.

(9) Baumann, L., Wernig, F., Born, S., and Oreb, M. (2020) Engineering Saccharomyces cerevisiae for production of fatty acids and their derivatives., in The Mycota Vol. II: Genetics and Biotechnology, 3rd edition (Benz, J. P., and Schipper, K., Eds.), pp 339-368. Springer, Switzerland.

(10) Knoll, L. J., Johnson, D. R., and Gordon, J. I. (1994) Biochemical Studies of Three Saccharomyces cerevisiae Acyl-CoA Synthetases, Faalp, FaaZp, and Faa3p*. J. Biol. Chem. 269, 16348-16356.

(11) Leber, C., Choi, J. W., Polson, B., and Da Silva, N. A. (2016) Disrupted short chain specific $\beta$ - 
oxidation and improved synthase expression increase synthesis of short chain fatty acids in Saccharomyces cerevisiae. Biotechnol. Bioeng. 113, 895-900.

(12) Henritzi, S., Fischer, M., Grininger, M., Oreb, M., and Boles, E. (2018) An engineered fatty acid synthase combined with a carboxylic acid reductase enables de novo production of 1-octanol in Saccharomyces cerevisiae. Biotechnol. Biofuels 11

(13) Lin, J.-L., Wagner, J. M., and Alper, H. S. (2017) Enabling tools for high-throughput detection of metabolites: Metabolic engineering and directed evolution applications. Biotechnol. Adv. 35, 950-970.

(14) Schallmey, M., Frunzke, J., and Eggeling, L. (2014) Looking for the pick of the bunch: highthroughput screening of producing microorganisms with biosensors. Curr. Opin. Biotechnol. 26, 148-154.

(15) Shi, S., Ang, E. L., and Zhao, H. (2018) In vivo biosensors: mechanisms, development, and applications. J. Ind. Microbiol. Biotechnol. 45, 491-516.

(16) Adeniran, A., Sherer, M., and Tyo, K. E. J. (2014) Yeast-based biosensors: design and applications. FEMS Yeast Res. 15, 1-15.

(17) D'Ambrosio, V., and Jensen, M. K. (2017) Lighting up yeast cell factories by transcription factor-based biosensors. FEMS Yeast Res. 17, 103-7.

(18) Baumann, L., Rajkumar, A. S., Morrissey, J. P., Boles, E., and Oreb, M. (2018) A yeast-based biosensor for screening of short- and medium-chain fatty acid production. ACS Synth. Biol. 7, 2640-2646.

(19) Piper, P., Mahé, Y., Thompson, S., Pandjaitan, R., Holyoak, C., Egner, R., Mühlbauer, M., Coote, P., and Kuchler, K. (1998) The pdr12 ABC transporter is required for the development of weak organic acid resistance in yeast. EMBO J. 17, 4257-4265.

(20) Kren, A., Mamnun, Y. M., Bauer, B. E., Schüller, C., Wolfger, H., Hatzixanthis, K., Mollapour, M., Gregori, C., Piper, P., and Kuchler, K. (2003) War1p, a novel transcription factor controlling weak acid stress response in yeast. Mol. Cell. Biol. 23, 1775-85.

(21) Williams, T. C., Xu, X., Ostrowski, M., Pretorius, I. S., and Paulsen, I. T. (2017) Positivefeedback, ratiometric biosensor expression improves high-throughput metabolite-producer screening efficiency in yeast. Synth. Biol. 2.

(22) Dabirian, Y., Gonçalves Teixeira, P., Nielsen, J., Siewers, V., and David, F. (2019) FadR-Based Biosensor-Assisted Screening for Genes Enhancing Fatty Acyl-CoA Pools in Saccharomyces cerevisiae. ACS Synth. Biol. 8, 1788-1800.

(23) Anderson, M. J., Barker, S. L., Boone, C., and Measday, V. (2012) Identification of RCN1 and RSA3 as ethanol-tolerant genes in Saccharomyces cerevisiae using a high copy barcoded library. FEMS Yeast Res. 12, 48-60.

(24) Shi, S., Ji, H., Siewers, V., and Nielsen, J. (2016) Improved production of fatty acids by Saccharomyces cerevisiae through screening a cDNA library from the oleaginous yeast Yarrowia lipolytica. FEMS Yeast Res. 16, 108. 
(25) Jones, G. M., Stalker, J., Humphray, S., West, A., Cox, T., Rogers, J., Dunham, I., and Prelich, G. (2008) A systematic library for comprehensive overexpression screens in Saccharomyces cerevisiae. Nat. Methods 5, 239-241.

(26) Wernig, F., Born, S., Boles, E., Grininger, M., and Oreb, M. (2020) Fusing $\alpha$ and $\beta$ subunits of the fungal fatty acid synthase leads to improved production of fatty acids. Sci. Rep. 10, 9780.

(27) Baxter, S. M., Rosenblum, J. S., Knutson, S., Nelson, M. R., Montimurro, J. S., Di Gennaro, J. A., Speir, J. A., Burbaum, J. J., and Fetrow, J. S. (2004) Synergistic computational and experimental proteomics approaches for more accurate detection of active serine hydrolases in yeast. Mol. Cell. Proteomics 3, 209-225.

(28) Saiardi, A., Erdjument-Bromage, H., Snowman, A. M., Tempst, P., and Snyder, S. H. (1999) Synthesis of diphosphoinositol pentakisphosphate by a newly identified family of higher inositol polyphosphate kinases. Curr. Biol. 9, 1323-1326.

(29) Dubois, E., Scherens, B., Vierendeels, F., Ho, M. M. W., Messenguy, F., and Shears, S. B. (2002) In Saccharomyces cerevisiae, the inositol polyphosphate kinase activity of Kcs1p is required for resistance to salt stress, cell wall integrity, and vacuolar morphogenesis. J. Biol. Chem. 277, 2375523763.

(30) Huh, W. K., Falvo, J. V., Gerke, L. C., Carroll, A. S., Howson, R. W., Weissman, J. S., and O'Shea, E. K. (2003) Global analysis of protein localization in budding yeast. Nature 425, 686-691.

(31) Ramachandran, G., Chidambaram, R., and Nachiappan, V. (2020) FSH1 encodes lysophospholipase activity in Saccharomyces cerevisiae. Biotechnol. Lett. 1-8.

(32) Saiardi, A., Caffrey, J. J., Snyder, S. H., and Shears, S. B. (2000) The inositol hexakisphosphate kinase family. Catalytic flexibility and function in yeast vacuole biogenesis. J. Biol. Chem. 275, 24686-24692.

(33) Bennett, M., Onnebo, S. M. N., Azevedo, C., and Saiardi, A. (2006) Inositol pyrophosphates: Metabolism and signaling. Cell. Mol. Life Sci. 63, 552-564.

(34) Generoso, W. C., Gottardi, M., Oreb, M., and Boles, E. (2016) Simplified CRISPR-Cas genome editing for Saccharomyces cerevisiae. J. Microbiol. Methods 127, 203-205.

(35) Gibson, D. G., Young, L., Chuang, R.-Y., Venter, J. C., Hutchison lii, C. A., and Smith, H. O. (2009) Enzymatic assembly of DNA molecules up to several hundred kilobases. Nat. Methods 6, 343-345.

(36) Gietz, R. D., and Schiestl, R. H. (2007) High-efficiency yeast transformation using the LiAc/SS carrier DNA/PEG method. Nat. Protoc. 2, 31-34.

(37) Bruder, S., Reifenrath, M., Thomik, T., Boles, E., and Herzog, K. (2016) Parallelised online biomass monitoring in shake flasks enables efficient strain and carbon source dependent growth characterisation of Saccharomyces cerevisiae. Microb. Cell Fact. 15, 1-15.

(38) Sambrook, J., Fritsch, E., and Maniatis, T. (1989) Molecular cloning: A laboratory manual. Second edition. Cold Spring Harb. Lab. Press. New York. (Evans, G. A., Ed.).

(39) Ichihara, K., and Fukubayashi, Y. (2010) Preparation of fatty acid methyl esters for gas-liquid chromatography. J. Lipid Res. 51, 635-640. 


\section{SUPPORTING INFORMATION}

Table S1. Plasmids used in this study.

\begin{tabular}{|c|c|c|}
\hline Plasmid & Characteristics & Reference \\
\hline \multicolumn{3}{|l|}{ Plasmids from library } \\
\hline pGP564 (“control”) & $2 \mu, L E U 2$, KanR & 1 \\
\hline Library & $\begin{array}{l}\text { pGP564, yeast genomic DNA fragments of } \sim 10 \\
\text { kb size }\end{array}$ & 1 \\
\hline Plate A10, well F1 (“FAS1”) & pGP564, [YKL183C-A $]^{*},[$ LOT5], FAS1, [PRS1]* & 1 \\
\hline Plate A16, well E2 (“FAS2”) & pGP564, [SSO1]\&, FAS2, YPL230W, [YPL229W]* & 1 \\
\hline Plate A1, well F6 (“F7-E2.8”) & $\begin{array}{l}\text { pGP564, [YBLO46W]\&, COR1, YBLO44W, } \\
\text { ECM13, FUI1, [PRE7]* }\end{array}$ & 1 \\
\hline Plate A13, well A3 (“F7-E3.1”) & $\begin{array}{l}\text { pGP564, [ESC1]\&, ERG8, FMP42, FSH2, UBP8, } \\
\text { MRE11, MRPL44, YMR226C, TAF7, [MTF1] }\end{array}$ & 1 \\
\hline Plate A1, well C1 (“F8-E2.4”) & pPGP564, GDH3, [YAL061W]* & 1 \\
\hline Plate A3, well E7 (“F8-E2.7”) & pPGP564, [PSF1], RAD61, HED1, DAD1, KCS1 & 1 \\
\hline Plate A12, well G7 (“F8-E2.10”) & $\begin{array}{l}\text { pPGP564, [YMLO23C]*, APT1, UNG1, } \\
\text { YMLO20W, OST6, YMLO18C, [PSP2]* }\end{array}$ & 1 \\
\hline $\begin{array}{l}\text { Plate A13, well B3 ("TAF7- } \\
\text { MTF1") }\end{array}$ & $\begin{array}{l}\text { pPGP564, [YMR226C]*, TAF7, MTF1, RRP5, } \\
\text { RPS10B, YMR230W-A, [PEP5]* }\end{array}$ & 1 \\
\hline \multicolumn{3}{|l|}{ Plasmids for CRISPR } \\
\hline $\mathrm{pRCC}-\mathrm{K}$ & $\begin{array}{l}2 \mu, k_{a n M X} \text { AmpR, pROX3-Cas9 }{ }^{o p t}-t C Y C 1, \\
p S N R 52-g R N A-t S U B 4\end{array}$ & 2 \\
\hline $\mathrm{pRCC}-\mathrm{N}$ & $\begin{array}{l}2 \mu, \text { natNT2, AmpR, pROX3-Cas9opt }-t C Y C 1, \\
p S N R 52-g R N A-t S U B 4\end{array}$ & 2 \\
\hline pRCC-K-LBV32 & $\begin{array}{l}\text { pRCC-K with gRNA for } p W A R 1 \text { locus } \\
\text { (TTGCTCCTACATTTATCGGA) }\end{array}$ & This study \\
\hline pRCC-N-LBV47 & $\begin{array}{l}\text { pRCC-N with gRNA for URA3 locus } \\
\text { (AACGTTACAGAAAAGCAGGC) }\end{array}$ & This study \\
\hline pRCC-K-LBV103 & $\begin{array}{l}\text { pRCC-K with gRNA for } P Y K 2 \text { locus } \\
\text { (ATGTCTTTGGCGGACAAGGG) }\end{array}$ & 3 \\
\hline pRCC-N-SHV42 & $\begin{array}{l}\text { pRCC-N with gRNA for FAA2 locus } \\
\text { (GAAGATTTTGAAACCTTACG) }\end{array}$ & 4 \\
\hline \multicolumn{3}{|l|}{ Other plasmids } \\
\hline LBV14 & $2 \mu, U R A 3, A m p R, p P D R 12-E n v y G F P$-tCYC1 & 3 \\
\hline LBV91 (“[PSF1]-RAD61”) & pGP564, [PSF1], RAD61 & This study \\
\hline LBV92 (“HED1-DAD1-KCS1") & pGP564, HED1, DAD1, KCS1 & This study \\
\hline LBV96 (“[ESC1]\&-ERG8”) & pGP564, [ESC1]\&, ERG8 & This study \\
\hline LBV97 (“FMP42-FSH2”) & pGP564, FMP42, FSH2 & This study \\
\hline LBV98 (“UBP8-MRE11”) & pGP564, UBP8, MRE11 & This study \\
\hline LBV99 (“MRPL44-YMR226C") & pGP564, MRPL44, YMR226C & This study \\
\hline LBV104 (“HED1”) & pGP564, HED1 & This study \\
\hline LBV105 (“DAD1") & pGP564, DAD1 & This study \\
\hline LBV106 (“KCS1”) & pGP564, KCS1 & This study \\
\hline LBV111 (“FMP42") & pGP564, FMP42 & This study \\
\hline LBV112 (“FSH2”) & pGP564, FSH2 & This study \\
\hline LBV113 (“KCS1-FSH2”) & pGP564, KCS1, FSH2 & This study \\
\hline LBGV023 & $\begin{array}{l}\text { ConLS'-gfp dropout-ConRE'-LEU2-CEN6-ARS4- } \\
\text { KanR-ColE1 }\end{array}$ & 5 \\
\hline FWV26 & pRS313, CEN6-ARS4, AmpR, HIS3 & 6 \\
\hline
\end{tabular}




\begin{tabular}{|l|l|l|}
\hline RPB34 (“FAS1") & $\begin{array}{l}\text { pRS315, CEN6-ARS4, AmpR, LEU2, pFAS1- } \\
\text { FAS1wt }\end{array}$ & 6 \\
\hline RPB36 (“FAS1 & $\begin{array}{l}\text { RR") } \\
\text { FAS315, CEN6-ARS4, AmpR, LEU2, pFAS1- }\end{array}$ & 6 \\
\hline RPB38 (“FAS2") & $\begin{array}{l}\text { pRS313, CEN6-ARS4, AmpR, HIS3, pFAS2- } \\
\text { FAS2wt }\end{array}$ & 6 \\
\hline
\end{tabular}

Table S2. Oligonucleotides used in this study.

\begin{tabular}{|c|c|c|}
\hline Primer & Sequence $5^{\prime}-3^{\prime}$ & Application \\
\hline \multicolumn{3}{|c|}{ Insertion of biosensor in ura3 locus } \\
\hline MRP130 & CGCCTGCTTTTCTGTAACGTTGATCATTTATCTTTCACTGCGGAG & \multirow{4}{*}{$\begin{array}{l}\text { Amplification of pRCC-N } \\
\text { with gRNA sequence for } \\
\text { cutting in ura3 }\end{array}$} \\
\hline MRP131 & $\begin{array}{l}\text { AACGTTACAGAAAAGCAGGCGTTTTAGAGCTAGAAATAGCAAGT } \\
\text { TAAAATAAGG }\end{array}$ & \\
\hline WGP234 & $\begin{array}{l}\text { CTTGGTGGTGTTCGTCGTATCTCTTAATCATAGAAGCAGACAATG } \\
\text { GAG }\end{array}$ & \\
\hline WGP235 & TGTTGTCTGACATTTTGAGAGTTAACACCGAAATTACCAAGGCTC & \\
\hline LBP195 & $\begin{array}{l}\text { GTATACATGCATTTACTTATAATACAGTTTTGATATCTTTGTTTTGC } \\
\text { ATTTTACATTC }\end{array}$ & \multirow{2}{*}{$\begin{array}{l}\text { Amplification of } \\
\text { biosensor ( } p P D R 12- \\
\text { EnvyGFP-tCYC1) from } \\
\text { LBY27 genomic DNA }\end{array}$} \\
\hline LBP196 & CTTTAATTTGCGGCCGGTACCCAATTCGCC & \\
\hline MRP141 & AACGCATGAAATCCTTCATTTG & \multirow{2}{*}{$\begin{array}{l}\text { Amplification for proof } \\
\text { of successful integration }\end{array}$} \\
\hline MRP142 & CGAAGGAAGGAGCACAGAC & \\
\hline \multicolumn{3}{|c|}{ Replacement of $p W A R 1$ by $p P D R 12$} \\
\hline WGP234 & $\begin{array}{l}\text { CTTGGTGGTGTTCGTCGTATCTCTTAATCATAGAAGCAGACAATG } \\
\text { GAG }\end{array}$ & \multirow{4}{*}{$\begin{array}{l}\text { Amplification of } \mathrm{pRCC}-\mathrm{K} \\
\text { with gRNA sequence for } \\
\text { cutting in } p W A R 1\end{array}$} \\
\hline WGP235 & TGTTGTCTGACATTTTGAGAGTTAACACCGAAATTACCAAGGCTC & \\
\hline LBP199 & $\begin{array}{l}\text { TTGCTCCTACATTTATCGGAGTTTTAGAGCTAGAAATAGCAAGTT } \\
\text { AAAATAAGG }\end{array}$ & \\
\hline LBP200 & TCCGATAAATGTAGGAGCAAGATCATTTATCTTTCACTGCGGAG & \\
\hline LBP201 & $\begin{array}{l}\text { GAATTCTGTTGTTGTAATTGTCATAACTATTGAGCGATATCTTTGT } \\
\text { TTTGCATTTTAC }\end{array}$ & \multirow{2}{*}{$\begin{array}{l}\text { Amplification of } p P D R 12 \\
\text { with overhangs to } \\
p W A R 1 \text { up- and } \\
\text { downstream regions }\end{array}$} \\
\hline LBP202 & $\begin{array}{l}\text { GACGGCAACGCCAGTTATTGCAATCTGCGTGTCCATTTTTTTATTA } \\
\text { ATAAGAACAATAAC }\end{array}$ & \\
\hline LBP203 & GACGCCACTGATATAAATCG & \multirow{2}{*}{$\begin{array}{l}\text { Amplification for proof } \\
\text { of successful integration }\end{array}$} \\
\hline LBP97 & GCTTCATAATAGTTCCTCTGG & \\
\hline \multicolumn{3}{|c|}{ Knockout of $F A A 2$} \\
\hline RPP266 & GAAGTCCCGGTGTCCCTGACGTTATTGTAG & \multirow{2}{*}{$\begin{array}{l}\text { Amplification donor DNA } \\
\text { for faa2 knockout from } \\
\text { SHY34 genomic DNA \& } \\
\text { proof of successful } \\
\text { knockout }\end{array}$} \\
\hline RPP267 & GTGACCCATGTACTCCGCTAGATTGACCAG & \\
\hline SHP80 & TTAGCCGGTTACACCAAAGG & \multirow{2}{*}{$\begin{array}{l}\text { Amplification of an } \\
\text { internal part of } F A A 2\end{array}$} \\
\hline LBP275 & GCCACGAATTTGCAGTTC & \\
\hline \multicolumn{3}{|c|}{ Cloning of LBV91-92, 96-99, 104-106, 111-113 } \\
\hline LBP375 & GTGGCGGCCGCTCTAGAACTAGTGGATC & \multirow{2}{*}{$\begin{array}{l}\text { Amplification of } \\
\text { fragment for LBV91 from } \\
\text { E2-F8.7 }\end{array}$} \\
\hline LBP376 & $\begin{array}{l}\text { TATCGAATTCCTGCAGCCCGGGGGATCTCGTCTACCCGAAGTACT } \\
\text { CTAGGCTTCCTATGC }\end{array}$ & \\
\hline LBP377 & $\begin{array}{l}\text { CGGTGGCGGCCGCTCTAGAACTAGTGGATCTCCGCACCTTTTAAA } \\
\text { AAAGGTTGAAAGGGC }\end{array}$ & \multirow{2}{*}{$\begin{array}{l}\text { Amplification of } \\
\text { fragment for LBV92 from } \\
\text { E2-F8.7 }\end{array}$} \\
\hline LBP393 & CGGTATCGATAAGCTTGATATCGAATTCC & \\
\hline
\end{tabular}




\begin{tabular}{|c|c|c|}
\hline LBP383 & $\begin{array}{l}\text { GCTTGATATCGAATTCCTGCAGCCCGGGGGATCGTGCACACTTTC } \\
\text { AAGCTAACACGCAC }\end{array}$ & \multirow{2}{*}{$\begin{array}{l}\text { Amplification of } \\
\text { fragment for LBV96 from } \\
\text { E3.F7.1 }\end{array}$} \\
\hline LBP390 & GGCGGCCGCTCTAGAACTAGTG & \\
\hline LBP384 & $\begin{array}{l}\text { CACCGCGGTGGCGGCCGCTCTAGAACTAGTGGATCCTTAAGGTA } \\
\text { GATAATAGTGGTCCAT }\end{array}$ & \multirow{2}{*}{$\begin{array}{l}\text { Amplification of } \\
\text { fragment for LBV97 from } \\
\text { E3.F7.1 }\end{array}$} \\
\hline LBP385 & $\begin{array}{l}\text { GTGAGCGCGCGTAATACGACTCACTATAGGAATTATTAATAACAA } \\
\text { ATAAAAAAAGCAGGG }\end{array}$ & \\
\hline LBP386 & $\begin{array}{l}\text { GCGGTGGCGGCCGCTCTAGAACTAGTGGATCGGTTACAGCTATT } \\
\text { AAATCTTATAGTCTTG }\end{array}$ & \multirow{2}{*}{$\begin{array}{l}\text { Amplification of } \\
\text { fragment for LBV98 from } \\
\text { E3.F7.1 }\end{array}$} \\
\hline LBP387 & $\begin{array}{l}\text { GCTTGATATCGAATTCCTGCAGCCCGGGGGATCGAAAATAAAGG } \\
\text { CATCTACAAATCTCAT }\end{array}$ & \\
\hline LBP388 & $\begin{array}{l}\text { GCTCCACCGCGGTGGCGGCCGCTCTAGAACTAGTGGATCAGTCG } \\
\text { AGTTTTATCGGATCTG }\end{array}$ & \multirow{2}{*}{$\begin{array}{l}\text { Amplification of } \\
\text { fragment for LBV99 from } \\
\text { E3.F7.1 }\end{array}$} \\
\hline LBP394 & $\begin{array}{l}\text { GTATCGATAAGCTTGATATCGAATTCCGTTCTCTTTATCATCTATA } \\
\text { TATTACTCTTATAC }\end{array}$ & \\
\hline LBP377 & $\begin{array}{l}\text { CGGTGGCGGCCGCTCTAGAACTAGTGGATCTCCGCACCTTTTAAA } \\
\text { AAAGGTTGAAAGGGC }\end{array}$ & \multirow{2}{*}{$\begin{array}{l}\text { Amplification of } \\
\text { fragment for LBV104 } \\
\text { from E2-F8.7 }\end{array}$} \\
\hline LBP410 & $\begin{array}{l}\text { GTCGACGGTATCGATAAGCTTGATATCGAATTCAGTTGCGGTTCC } \\
\text { CTCTGTCTCTC }\end{array}$ & \\
\hline LBP411 & $\begin{array}{l}\text { CCACCGCGGTGGCGGCCGCTCTAGAACTAGTGGTTCAAAGAGGA } \\
\text { GAACGTTTG }\end{array}$ & \multirow{2}{*}{$\begin{array}{l}\text { Amplification of } \\
\text { fragment for LBV105 } \\
\text { from E2-F8.7 }\end{array}$} \\
\hline LBP412 & $\begin{array}{l}\text { GTATCGATAAGCTTGATATCGAATTCAAAGAAAGGATAGAACTA } \\
\text { ATGAATATTCT }\end{array}$ & \\
\hline LBP413 & $\begin{array}{l}\text { CCGCGGTGGCGGCCGCTCTAGAACTAGTGATGTACATATATCCTC } \\
\text { ACACGTCCG }\end{array}$ & \multirow{2}{*}{$\begin{array}{l}\text { Amplification of } \\
\text { fragment for LBV106 } \\
\text { from E2-F8.7 }\end{array}$} \\
\hline LBP393 & CGGTATCGATAAGCTTGATATCGAATTCC & \\
\hline LBP384 & $\begin{array}{l}\text { CACCGCGGTGGCGGCCGCTCTAGAACTAGTGGATCCTTAAGGTA } \\
\text { GATAATAGTGGTCCAT }\end{array}$ & \multirow{2}{*}{$\begin{array}{l}\text { Amplification of } \\
\text { fragments for LBV111 } \\
\text { from E3-F7.1 }\end{array}$} \\
\hline LBP431 & $\begin{array}{l}\text { CTGACACAATGGACAATTAAATAAAATTAAGTAAAAAAAATAAG } \\
\text { GACTTTAATTTTTAAACGGTGAGTAAGGAAAG }\end{array}$ & \\
\hline LBP385 & $\begin{array}{l}\text { GTGAGCGCGCGTAATACGACTCACTATAGGAATTATTAATAACAA } \\
\text { ATAAAAAAAGCAGGG }\end{array}$ & \multirow{2}{*}{$\begin{array}{l}\text { Amplification of } \\
\text { fragment for LBV112 } \\
\text { from E3-F7.1 }\end{array}$} \\
\hline LBP433 & $\begin{array}{l}\text { CACCGCGGTGGCGGCCGCTCTAGAACTAGTGTACAGTCTTTACTG } \\
\text { CCCTAATA }\end{array}$ & \\
\hline LBP413 & $\begin{array}{l}\text { CCGCGGTGGCGGCCGCTCTAGAACTAGTGATGTACATATATCCTC } \\
\text { ACACGTCCG }\end{array}$ & \multirow{4}{*}{$\begin{array}{l}\text { Amplification of } \\
\text { fragments for LBV113 } \\
\text { from E2-F8.7 and E3-F7.1 }\end{array}$} \\
\hline LBP436 & $\begin{array}{l}\text { GGCCAGTATTAGGGCAGTAAAGACTGTACGAAAAACACTTTTCT } \\
\text { GTTCTTGTTTGTC }\end{array}$ & \\
\hline LBP385 & $\begin{array}{l}\text { GTGAGCGCGCGTAATACGACTCACTATAGGAATTATTAATAACAA } \\
\text { ATAAAAAAAGCAGGG }\end{array}$ & \\
\hline LBP437 & $\begin{array}{l}\text { GACAAACAAGAACAGAAAAGTGTTTTTCGTACAGTCTTTACTGCC } \\
\text { CTAATA }\end{array}$ & \\
\hline
\end{tabular}




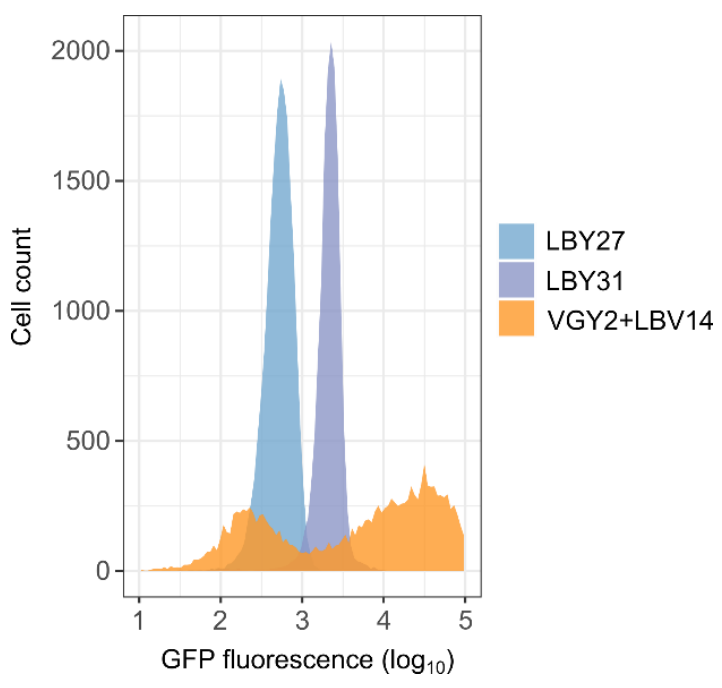

Figure S1. Fluorescence signal of genomically integrated vs. plasmid-based biosensor. The two strains LBY27 and LBY31 contain a stable genomic integration of the biosensor pPDR12-GFP whereas VGY2 was transformed with a multi-copy plasmid (LBV14) containing PPDR12-GFP. All strains were inoculated to an $\mathrm{OD}_{600}=0.1$ and grown in buffered SCD medium. Aliquots were taken after $4 \mathrm{~h}$ for GFP fluorescence measurement in a flow cytometer (common logarithmic transformation of fluorescence area plotted). LBY27 (non-producer). VGY2 (producer): FAS1 ${ }^{R K}$. LBY31 (producer): FAS1 ${ }^{R K}, \triangle p W A R 1:: p P D R 12$.

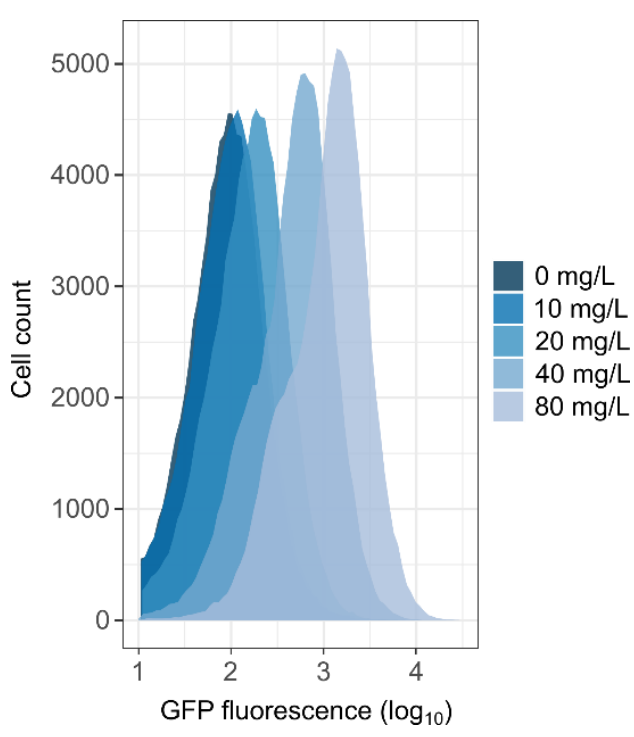

Figure S2. Evaluation of biosensor response to supplemented octanoic acid. Strain LBY27, which contains a stable integration of the biosensor PPDR12-GFP, was inoculated to an $\mathrm{OD}_{600}=0.1$ and cultivated until stationary phase. The indicated octanoic acid amounts were added and the cultures were cultivated for another $3 \mathrm{~h}$ before measurement of GFP fluorescence in a flow cytometer (common logarithmic transformation of fluorescence area plotted). 


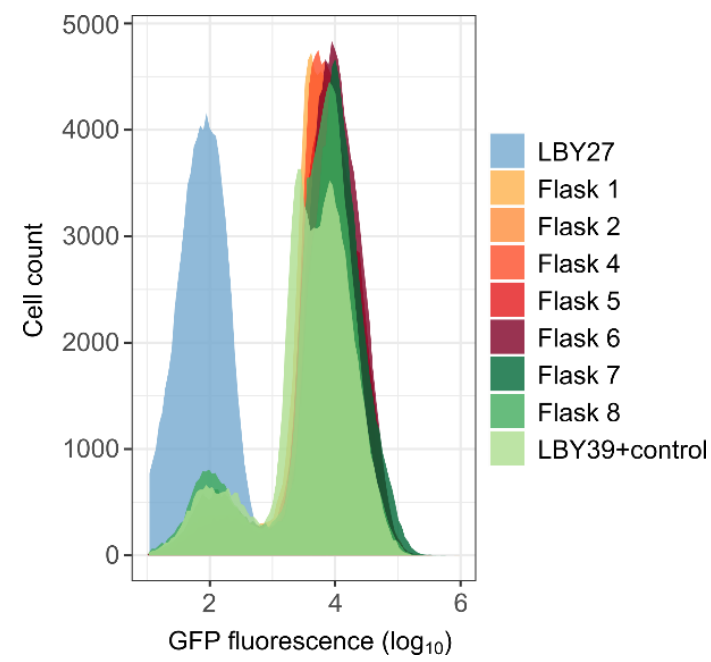

Figure S3. Fluorescence signals of library populations before the first enrichment (E1 sorting). Strain LBY27 (non-producer) and LBY39+control (producer + empty vector) serve as controls. All contain a stable integration of the biosensor pPDR12-GFP. Populations of Flask1-8 (LBY39 with library) were grown for $48 \mathrm{~h}$ in buffered SCD medium before measurement in a flow cytometer (common logarithmic transformation of fluorescence area plotted). Of the depicted populations, the top $10 \%$ of fluorescent events were sorted. LBY39: FAS1 ${ }^{R K}, \triangle$ faa 2 .

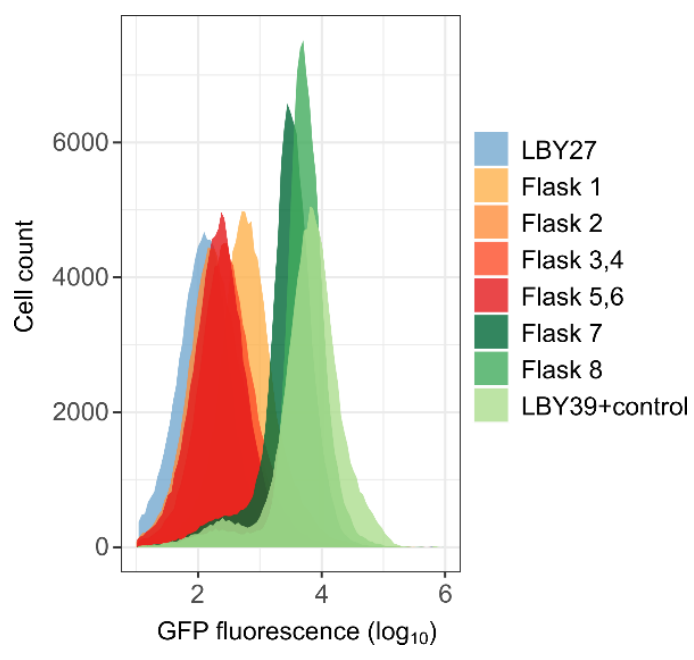

Figure S4. Fluorescence signals of library populations after the first enrichment (E2 sorting). Strain LBY27 (non-producer) and LBY39+control (producer + empty vector) serve as controls. All contain a stable integration of the biosensor pPDR12-GFP. Populations of Flask1-8 (LBY39 with library) were grown for $66 \mathrm{~h}$ in buffered SCD medium before measurement of GFP fluorescence in a flow cytometer (common logarithmic transformation of fluorescence area plotted). Of the depicted populations, the top $7.5 \%$ of fluorescent events were sorted. LBY39: FAS1 ${ }^{R K}, \triangle f a a 2$. 


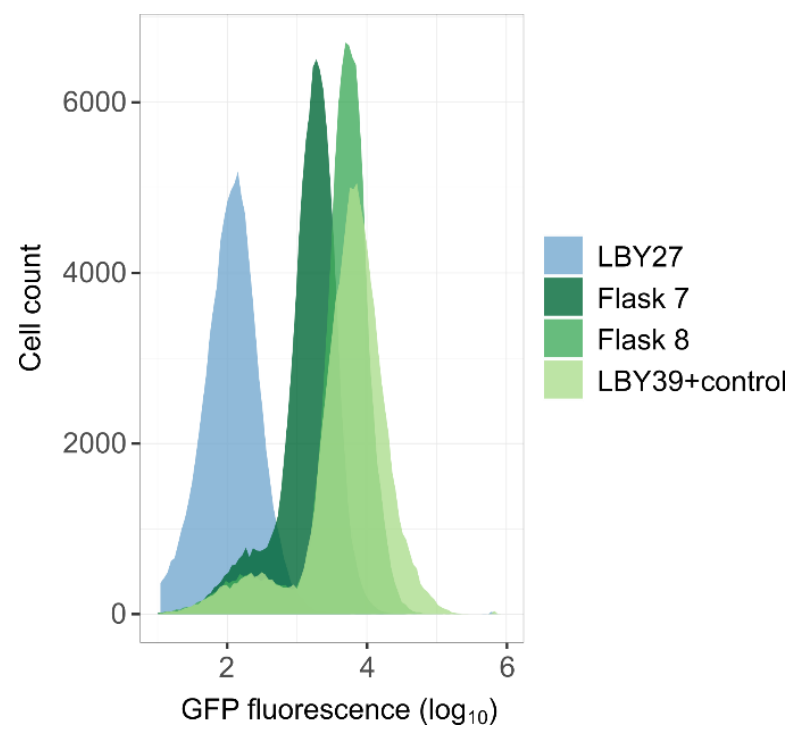

Figure S5. Fluorescence signals of library populations after the second enrichment (E3 sorting). Strain LBY27 (non-producer) and LBY39+control (producer + empty vector) serve as controls. All contain a stable integration of the biosensor PPDR12-GFP. Populations of Flask7-8 (LBY39 with library) were grown for $66 \mathrm{~h}$ in buffered SCD medium before measurement of GFP fluorescence in a flow cytometer (common logarithmic transformation of fluorescence area plotted). Of the depicted populations, the top $5 \%$ and top $1 \%$, respectively, of fluorescent events were sorted. LBY39: FAS1 ${ }^{R K}, \triangle f a a 2$.

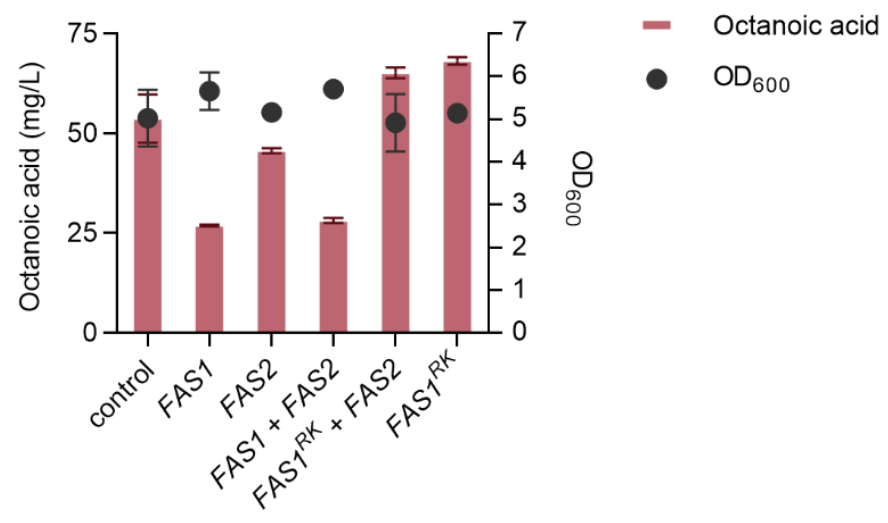

Figure S6. Effect of additional expression of FAS genes in LBY38 (FAS1 $\left.{ }^{R K}\right)$ background strain. $\mathrm{OD}_{600}$ (dots) and octanoic acid titers (bars) of octanoic acid producer strain LBY38 with plasmids containing indicated genes (+ empty plasmid LBGV023 or FWV26, for those with only one gene indicated). As control served LBY38 carrying empty vectors FWV26 and LBGV023. Fatty acids were extracted, methylated and quantified via GC $72 \mathrm{~h}$ after inoculation in buffered SCD-leu-his medium. LBY38: $F A S 1^{R K}, \triangle$ faa2. $n=2$, error bars $= \pm \mathrm{SD}$. 
A)

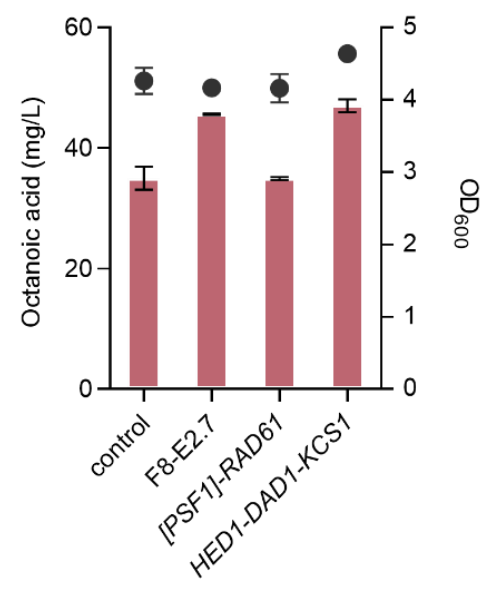

B)

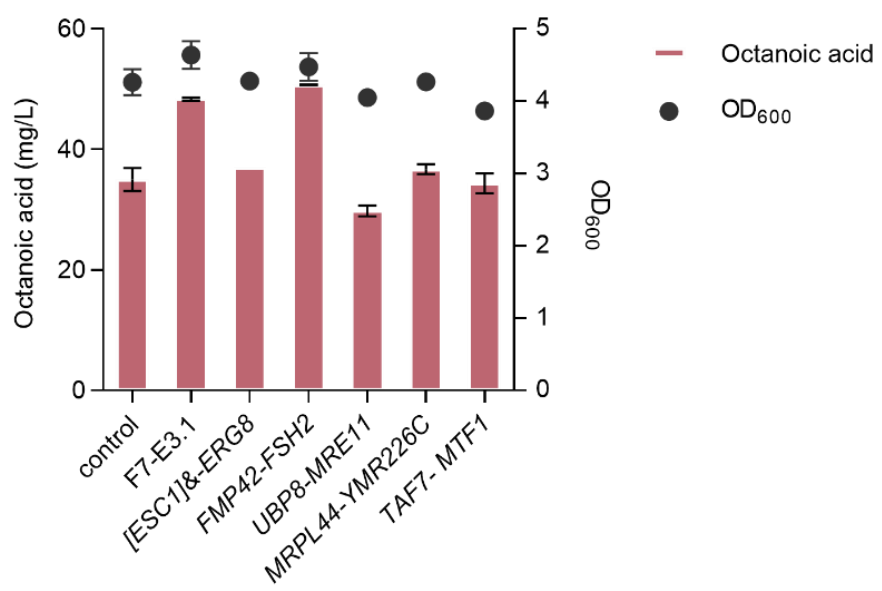

Figure S7. Identification of genes increasing octanoic acid titers and growth. OD 600 (dots) and octanoic acid titers (bars) of octanoic acid producer strain LBY38 containing either the vector control or plasmids with the indicated genes. (A) Plasmid F8-E2.7 contains all five genes indicated on the X-axis (B) Plasmid F7-E3.1 contains all ten genes indicated on the $x$-axis. Fatty acids were extracted, methylated and quantified via GC $48 \mathrm{~h}$ after inoculation in buffered SCD medium. LBY38 (producer): FAS1 ${ }^{R K}, \triangle$ faa2. []: intact ORF, possibly missing up- or downstream regions. []\&: $5^{\prime}$ end of gene is missing. $n=2$, error bars $= \pm$ SD

\section{REFERENCES}

(1) Jones, G. M., Stalker, J., Humphray, S., West, A., Cox, T., Rogers, J., Dunham, I., and Prelich, G. (2008) A systematic library for comprehensive overexpression screens in Saccharomyces cerevisiae. Nat. Methods 5, 239-241.

(2) Generoso, W. C., Gottardi, M., Oreb, M., and Boles, E. (2016) Simplified CRISPR-Cas genome editing for Saccharomyces cerevisiae. J. Microbiol. Methods 127, 203-205.

(3) Baumann, L., Rajkumar, A. S., Morrissey, J. P., Boles, E., and Oreb, M. (2018) A yeast-based biosensor for screening of short- and medium-chain fatty acid production. ACS Synth. Biol. 7, 2640-2646.

(4) Henritzi, S., Fischer, M., Grininger, M., Oreb, M., and Boles, E. (2018) An engineered fatty acid synthase combined with a carboxylic acid reductase enables de novo production of 1-octanol in Saccharomyces cerevisiae. Biotechnol. Biofuels 11.

(5) Lee, M. E., DeLoache, W. C., Cervantes, B., and Dueber, J. E. (2015) A Highly Characterized Yeast Toolkit for Modular, Multipart Assembly. ACS Synth. Biol. 4, 975-986.

(6) Gajewski, J., Pavlovic, R., Fischer, M., Boles, E., and Grininger, M. (2017) Engineering fungal de novo fatty acid synthesis for short chain fatty acid production. Nat. Commun. 8, 14650. 


\section{Deutsche Zusammenfassung}

Octansäure (C8 Fettsäure) ist eine mittelkettige Fettsäure, die in der Natur vor allem in Palmkernöl und Kokosnüssen vorkommt. Sie wird in verschiedenen Produkten wie Reinigungsmitteln, Kosmetika, Pestiziden und Herbiziden, sowie in Lebensmitteln zur Konservierung oder zum Aromatisieren eingesetzt. Darüber hinaus wird die Anwendung von Octansäure zur medizinischen Behandlung, z. B. von hohen Cholesterinwerten, untersucht. Um die seit Jahren steigende Nachfrage zu decken, hat der Anbau von Öl- und Kokospalmen in den letzten Jahren kontinuierlich zugenommen. Der Anbau insbesondere von Ölpalmen erfolgt oft in großflächigen Monokulturen, für welche Regenwald abgeholzt wird. Die damit einhergehende Umweltproblematik ist zunehmend im Fokus der Öffentlichkeit und befördert die Suche nach umweltfreundlicheren Produktionsmethoden. Eine biotechnologische Produktion mit Mikroorganismen wie Hefe, stellt dabei eine attraktive, nachhaltigere Alternative dar.

Die Bäckerhefe Saccharomyces cerevisiae wird seit Jahrtausenden für die Herstellung von Brot, Wein und Bier genutzt. Heutzutage kann basierend auf umfassenden Kenntnissen über Stoffwechsel und Genetik, der Metabolismus der Hefe so verändert werden, dass die Produktion einer Vielzahl von industriell relevanten Produkten möglich ist. Zur Herstellung von Octansäure wurde die zytosolische Fettsäuresynthase (FAS, fatty acid synthase) von S. cerevisiae genutzt. Natürlicherweise produziert Hefe im FAS-Zyklus hauptsächlich langkettige Fettsäuren mit Kettenlängen von $\mathrm{C} 16$ und $\mathrm{C} 18$, und nur geringe Mengen mittelkettiger Fettsäuren (C8-C14). Um einen S. cerevisiae-Stamm mit einem veränderten Fettsäurespektrum zu erzeugen, wurden die FAS-kodierenden Gene entsprechend verändert. Die hierbei erzeugte Variante FAS ${ }^{\text {R1834K }}$ bringt die Hefe zur vermehrten Produktion von Octansäure (Gajewski et al., 2017). Die Hefe produziert Octansäure im FAS-Zyklus und entlässt diese zunächst in ihrer aktivierten Form, dem Octanoyl-CoA. Dieses wird anschließend durch Thioesterasen gespalten, die Octansäure freigesetzt, und von der Zelle ins

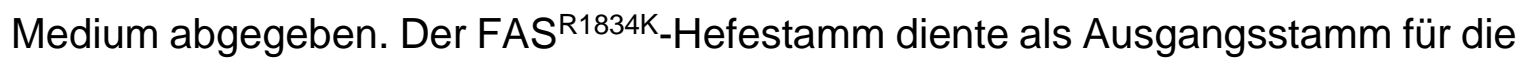
vorliegende Arbeit. 
Das Ziel dieser Arbeit war es, die Grundlagen für eine Verbesserung der Octansäureproduktion in Hefe zu schaffen. Die Erhöhung der Octansäuretiter und -ausbeuten ist die Voraussetzung für eine wirtschaftlich rentable Herstellung im industriellen Maßstab. Eine der Herausforderungen hierbei ist die arbeitsintensive, durchsatzarme Probenvorbereitung und -messung von der Octansäure aus den produzierenden Hefen. Um diese zu vereinfachen und beschleunigen, wurde im Rahmen der vorliegenden Arbeit ein Biosensor für Octansäure entwickelt. Biosensoren ermöglichen die Erkennung kleiner Moleküle in Hefezellen, worauf diese mit einer spezifischen Regulierung der Genexpression reagieren. Im Fall eines GFP (green fluorescent protein) -gekoppelten Biosensors wird dies durch eine Veränderung der Fluoreszenz messbar.

Der entwickelte Biosensor basiert auf dem pPDR12-Promotor, welcher durch den Transkriptionsfaktor War1 reguliert wird. Die Kopplung von pPDR12 mit GFP als Reportergen auf einem multicopy-Plasmid ermöglichte eine in vivo-Detektion durch Messung der Fluoreszenzintensität. Dadurch konnten mittels des Biosensors die kurz- und mittelkettigen Fettsäuren Hexan-, Heptan- und Octansäure schnell und einfach quantifiziert werden (Baumann et al., 2018). Dies ist der erste Biosensor, der sowohl zugefütterte Octansäure als auch im Kulturüberstand von Produzentenstämmen vorhandene Octansäure in einem hohen linearen und dynamischen Bereich messen kann. Die Funktionalität des Biosensors wurde durch die Korrelation des Biosensorsignals mit den gaschromatographisch bestimmten Octansäurekonzentrationen aus Kulturüberständen validiert. Es konnte gezeigt werden, dass der Biosensor Octansäure in einem linearen Bereich von 0,01-0,75 mM (1-110 mg/L) detektiert, was innerhalb des Produktionsspektrums des Ausgangsstammes liegt. Außerdem wurde die Fluoreszenzintensität durch Octansäureaktivierung um das bis zu 10-fache gesteigert, was ein hohes Reaktionsspektrum darstellt. Ein Biosensor mit den geschilderten Eigenschaften eignet sich, um große Stammbibliotheken zu screenen und dabei denjenigen Hefestamm zu identifizieren, der am meisten Octansäure produziert. 
Mit dem Biosensor wurde ein Hochdurchsatzscreening mit Hilfe der Durchflusszytomerie (FACS, fluorescence-activated cell sorting) durchgeführt (Baumann et al., 2020a). Die Methode ermöglicht es in Kombination mit dem Biosensor innerhalb weniger Minuten Millionen von Hefezellen zu analysieren. Für das FACS-Screening wurde der Biosensor an die veränderten Anforderungen angepasst. Zunächst konnte die genomische Integration des Biosensors in einen Octansäure-Produzentenstamm das Einzelzellrauschen drastisch reduzieren. Die Funktionalität dieses Biosensors konnte durch Zugabe von Octansäure im Konzentrationsbereich von 0-80 $\mathrm{mg} / \mathrm{L}$ und anschließender durchflusszytometrischer Analyse validiert werden. Des Weiteren wurde der Fettsäureabbau ( $\beta$-Oxidation) von mittelkettigen Fettsäuren erfolgreich durch die Deletion von $F A A 2$ verhindert. $F A A 2$ kodiert für eine Acyl-CoA Synthetase, welche für die Aktivierung von mittelkettigen Fettsäuren zur peroxisomalen $\beta$-Oxidation verantwortlich ist.

Mit dem angepassten Biosensorstamm wurde ein HochdurchsatzscreeningProtokoll entworfen, welches basierend auf Erkenntnissen aus verschiedenen Vorexperimenten optimiert wurde. So wurden im finalen Screening iterative Anreicherungen der Stämme mit der höchsten Fluoreszenz durchgeführt, um die Anzahl der falsch-positiven Stämme zu reduzieren. Für das Screening wurde der Biosensorstamm mit einer Bibliothek von multicopy-Plasmiden, die das gesamte Hefegenom abdeckte, transformiert. Ziel war es Gene zu finden, die wenn überexprimiert, die Octansäureproduktion steigern. Im FACS-Screening konnten zwei Gene, FSH2 und KCS1, identifizieren werden, die in kombinierter Überexpression die Octansäuretiter um 55 \% im Vergleich zum Ausgangsstamm erhöhten. Dies war der erste Bericht über einen Effekt von FSH2 und KCS1 auf die Octansäuretiter der Hefe. Die entwickelte Hochdurchsatzmethode kann auch für das Screening anderer genetischer Bibliotheken verwendet werden. Dies kann zukünftige Experimente zur Verbesserung von Hexansäure-, Heptansäure-, oder Octansäure-produzierenden Hefen deutlich beschleunigen.

Octansäure ist in hohen Konzentrationen für Hefezellen toxisch. Dies konnte in Wachstumstests mit $S$. cerevisiae-Stämmen bestätigen werden. Um robustere Produzentenstämme zu erzeugen wurden verschiedene Strategien evaluiert. Es 
wurde eine adaptive Evolvierung (ALE, adaptive laboratory evolution) mit einem Wildtypstamm, den wir in Anwesenheit von hohen Octansäurekonzentrationen kultivierten, durchgeführt. Nach der adaptiven Evolvierung über etwa 60 Generationen zeigte die gemischte Hefepopulation eine erhöhte Wachstumsrate im Vergleich zum Ausgangshefestamm. Dieser Wachstumsvorteil konnte jedoch bei der Anzucht einzelner Hefestämme nicht reproduziert werden. Möglicherweise ist ALE keine geeignete Methode, um die Robustheit der Hefe gegenüber Octansäure wesentlich und konstitutiv zu erhöhen. Eine alternative Strategie bestand darin, den Effekt einer Überexpression bzw. Deletion einiger Gene auf die Octansäuretoleranz des Produktionsstammes zu evaluieren. Hierzu gehörten Transporter- (PDR12, TPO1) und Transkriptionsfaktor-kodierende Gene (PDR1, $P D R 3$, WAR1) sowie das mutierte Acetyl-CoA-Carboxylase-kodierende Gen ACC151157A. Frühere Untersuchungen durch ähnliche Experimente mit diesen Genen hatten von einer erhöhten Toleranz der Hefe gegenüber Octansäure berichtet. Entgegen der Erwartung war eine erhöhte Robustheit in unserem $S$. cerevisiae-Stamm jedoch nicht zu beobachten. Dies legte die Vermutung nahe, dass der verwendete $S$. cerevisiae-Stamm von Natur aus eine niedrige Toleranz aufweist. In Wachstumstests mit Octansäure wurde daraufhin die Toleranz von fünf S. cerevisiae-Stämmen untersucht und tatsächlich von Natur aus robustere Stämme entdeckt. Diese Stämme, z. B. S. cerevisiae EthanolRed, könnten in Zukunft als Ausgangsstamm für weitere Optimierungen dienen.

Im Rahmen eines weiteren Projekts wurde erstmalig eine Transkriptomanalyse (RNA-Seq) eines S. cerevisiae-Octansäureproduzenten realisiert. Sie wurde im Vergleich zu einem Wildtypstamm ausgeführt und es wurden in drei unterschiedlichen Fermentationsphasen Proben entnommen (Baumann et al., 2021) Solch eine Analyse ermöglicht einen umfangreichen Einblick in die globale Genexpression. Des Weiteren wurden die gesammelten Genexpressionsdaten mit bereits veröffentlichten Daten verglichen, welche von zwei Forschergruppen stammten, die die Genexpression in Wildtyp-Stämmen in Reaktion auf zugefütterte Octansäure untersucht hatten. Die vergleichende Analyse ergab, dass sich die Genexpression in Folge von intrazellulär produzierter Octansäure deutlich von der Genexpression in Reaktion auf zugefütterte Octansäure unterscheidet. Darüber hinaus konnte im RNA-Seq Experiment ein negativer Effekt der 
Octansäureproduktion auf das Wachstum der Hefe bestätigt werden. Die Daten deuteten darauf hin, dass das reduzierte Wachstum des Octansäureproduzierenden Stammes - zumindest teilweise - auf eine verminderte Produktion von langkettigen Fettsäuren zurückzuführen sein könnte. Langkettige Fettsäuren erfüllen jedoch essentielle Funktionen in der Zelle, u. A. als Energiespeicher und Bestandteile von Zellmembranen. Basierend auf der vergleichenden Analyse der RNA-Seq-Daten der drei Fermentationsphasen wurden einzelne Gene zur Überexpression ausgewählt. Hierbei wurde das Gen RPL4OB, kodierend für eine Untereinheit des Ribosoms, identifiziert, dessen Überexpression zu einer Erhöhung der Octansäuretiter um 40 \% führte. Die Erkenntnis, dass Rpl40b eine Rolle in der Fettsäurebiosynthese spielt wurde hier erstmals berichtet - der genaue Mechanismus dieses Effekts ist allerdings noch unbekannt.

Zusammenfassend wurden in dieser Arbeit erfolgreich neue Strategien entwickelt und etablierte Methoden genutzt, um die Hefe $S$. cerevisiae als Zellfabrik für die Produktion von Octansäure zu verbessern. Ein Octansäure-Biosensor wurde konstruiert, verifiziert und für die Implementierung in einem Hochdurchsatz-FACSScreening optimiert. Die entwickelte Methode ist sehr vielseitig und kann für Screenings verschiedenster Stammbibliotheken von Produzenten kurz- und mittelkettiger Fettsäuren eingesetzt werden. Eine Transkriptomanalyse ergab ein umfassendes Bild der Stammphysiologie während der Octansäureproduktion. Drei Gene wurden identifiziert, die durch Überexpression die Octansäuretiter erhöhen.

Die entwickelten Strategien und identifizierten Gene sind für die zukünftige mikrobielle Octansäureproduktion von wichtiger Bedeutung. Gefundene Gene können in einem natürlicherweise robusten Stamm, wie den im Rahmen dieser Arbeit evaluierten $S$. cerevisiae EthanolRed überexprimiert und mit weiteren Stammoptimierungen kombiniert werden. Im Gegensatz zu derzeitigen Produktionsverfahren müssen für die Octansäureproduktion mit Hefe keine neuen Landflächen erschlossen werden. Vielmehr kann Hefe andere Substrate wie landwirtschaftliche Abfallstoffe, z. B. Lignocellulose, als Energiequelle und Ausgangssubstrat nutzen. 


\section{Danksagung}

Mein besonderer Dank gilt Prof. Dr. Eckhard Boles und Dr. Mislav Oreb für die Betreuung meiner Doktorarbeit. Danke, dass ihr mir das spannende Thema anvertraut habt und immer eine offene Tür für Fragen und wissenschaftliche Diskussionen hattet. Besonders möchte ich euch auch dafür danken, dass ihr neue Ideen unterstützt und mir die Freiheit gegeben habt diese in verschiedenen Kooperationsprojekten umzusetzen. Neben Frankfurt durfte ich in Basel, Göteborg und Darmstadt forschen, sowie eine Vielzahl an Konferenzen und CHASSY Meetings besuchen, danke dass ihr das ermöglicht habt.

Ebenfalls bedanke ich mich bei Prof. Dr. Claudia Büchel für die Übernahme des Zweitgutachtens.

Für die Finanzierung des Projekts CHASSY bedanke ich mich bei der Europäischen Union. Außerdem möchte ich mich für die gute Zusammenarbeit im Projekt bei allen Kolleg*innen von CHASSY bedanken ebenso wie für die erfolgreichen externen Kooperationen mit FGen sowie der AG Kabisch. Danke Stefan und Johannes, dass ihr mir euer Labor und Equipment zur Verfügung gestellt und euer Wissen weitervermittelt habt. Danke Andreas und dem FGen-Team, sowie Tyler, dass ihr meine Auslandsaufenthalte in Basel und Göteborg zu lehrreichen und besonderen Erlebnissen habt werden lassen.

Ich möchte mich bei der gesamten Arbeitsgruppe Boles für die schönen vier Jahre, die gute Arbeitsatmosphäre und die tollen gemeinsamen Unternehmungen bedanken. Muchas gracias a mis compas Sebastian y Fernando und Sina für die Unterstützung und die schöne gemeinsame Zeit.

Ein besonderer Dank geht an meine Familie und Freund*innen für die Unterstützung während der gesamten PhD-Zeit. 